Nuclear Physics A356 (1981) 146-222; (C) North-Holland Publishing Co., Amsterdam

Not to be reproduced by photoprint or microfilm without written permission from the publisher

\title{
SPECTROSCOPIC AMPLITUDES FOR COMPLEX CLUSTER SYSTEMS
}

\author{
K. T. $\mathrm{HECHT}^{\dagger}$ \\ Max-Planck-Institut für Kernphysik, Heidelberg, W. Germany \\ E. J. RESKE ${ }^{+\dagger}$ \\ Physics Dept., University of Michigan, Ann Arbor, MI 48109, USA
}

T. H. SELIGMAN

Instituto de Fisica, Universidad de México, Apdo. Postal 20-364, México 20, DF

and

W. ZAHN

Institut für Theoretische Physik der Universität Erlangen-Nürnberg, W. Germany

Received 24 June 1980

Abstract: By expanding the Bargmann-Segal integral transform of norm and overlap kernels in appropriately SU(3) coupled Bargmann space functions, the calculation of norm and overlap matrix elements in a cluster model basis is reduced to purely algebraic techniques involving the algebra of SU(3) recoupling transformations. This technique has been further developed to make calculations possible for systems of two heavy fragments other than closed-shell nuclei. In one application of the method, analytic expressions are given for the norms of binary fragment systems in which a light fragment of mass number $f, f \leqslant 4$, is combined with a heavy fragment of mass number $A-f$, with $A-f \leqslant 24$. The $A-f$ fragment nuclei with different p- and sd-shell structure illustrate somewhat different problems in the recoupling technique. In a second application, spectroscopic amplitudes are calculated for the most important open channels of the ${ }^{12} \mathrm{C}+{ }^{12} \mathrm{C}$ resonances. Eigenvalues and eigenvectors of the antisymmetrizer are evaluated in a "molecular basis" of the ${ }^{12} \mathrm{C}+{ }^{12} \mathrm{C}$ system, in which each ${ }^{12} \mathrm{C}$ nucleus is assumed to have SU(3) symmetry (04) with internal rotational excitations of $0^{+}, 2^{+}$and $4^{+}$. Reduced width amplitudes are calculated connecting such normalized, fully antisymmetrized molecular basis states to exit channels which include: $\alpha+{ }^{20} \mathrm{Ne}$ with ${ }^{20} \mathrm{Ne}$ internal functions of (80) SU(3) symmetry, $\left(K=0^{+}\right.$band), and (82) SU(3) symmetry, $(K=2-$ band $) ;{ }^{16} \mathrm{O}+{ }^{8} \mathrm{Be}$; and ${ }^{23} \mathrm{Na}+\mathrm{p}$ or ${ }^{23} \mathrm{Mg}+\mathrm{n}$ fragments with ${ }^{23} \mathrm{Na}$ or ${ }^{23} \mathrm{Mg}$ excitations in $K=\frac{3}{2}$ and $\frac{1}{2}$ rotational bands of SU(3) symmetry (83).

\section{Introduction}

In recent years refinements in the resonating group method ${ }^{1-3}$ ) have made possible a sound microscopic treatment of a number of nuclear reaction and structure prob-

+ US Senior Science Fellow of the Alexander von Humboldt Foundation.

${ }^{+\dagger}$ Supported in part by the US National Science Foundation.

‡ Supported by the Deutsche Forschungsgemeinschaft. 
lems. However, detailed applications to bound-state, scattering, and reaction problems have been limited mainly to very light nuclear systems ${ }^{3-7}$ ) or to scattering problems involving closed-shell nuclei such as $\alpha,{ }^{16} \mathrm{O}$, and $\left.{ }^{40} \mathrm{Ca}\left[\mathrm{refs} .{ }^{3,8-10}\right)\right]$. Many of the recent advances have been made possible by the introduction of integral transform techniques ${ }^{11-21}$ ) and the closely related complex generator coordinate technique ${ }^{3}$ ) which have reduced the computational effort in the calculation of resonating group kernels. Nevertheless, the evaluation of resonating group kernels in an angular momentum coupled basis has proved difficult for reaction problems involving heavy fragments other than closed-shell nuclei. By exploiting the SU(3) symmetry properties of the relative motion and internal harmonic oscillator functions of a cluster basis it may be possible to handle resonating group calculations involving heavy fragments other than closed-shell nuclei. This is true in particular if the cluster functions are expanded in an SU(3) coupled basis in which SU(3) recoupling techniques can be used to advantage, and in which the details of the coupling of channel spins to relative motion angular momenta can be avoided till the very final step of a calculation. Of the many integral transforms used in microscopic nuclear clustermodel calculations, the Bargmann-Segal (BS) transform ${ }^{22}$ ) is ideally suited to the exploitation of SU(3) recoupling techniques since oscillator functions have very simple properties in Bargmann space. The combination of the BS integral transform with SU(3) recoupling techniques can greatly reduce the computational difficulties in the calculation of resonating group kernels ${ }^{23}$ ). The potential of this technique has been illustrated with a few examples in ref. ${ }^{24}$ ). Details of the technique have also been demonstrated with the calculation of the norms for cluster systems made up of a heavy fragment and an $\alpha$-particle ${ }^{25,26}$ ) for states of arbitrarily high oscillator excitation in the relative motion degree of freedom. A very similar technique has recently also been used by Fujiwara and Horiuchi ${ }^{27}$ ) in their generator coordinate theory of norm kernels with applications to ${ }^{12} \mathrm{C}+\alpha,{ }^{12} \mathrm{C}+{ }^{16} \mathrm{O}$ and ${ }^{16} \mathrm{O}+\alpha+\alpha$. In their treatment the complex generator coordinate $\boldsymbol{R}$ corresponds to the Bargmannspace $\boldsymbol{K}$ variable of refs. ${ }^{23-26}$ ).

It is the purpose of the present work to expand the combined BS transform and SU(3) recoupling technique to facilitate calculations involving nuclear systems made up of at least two heavy fragments which are not closed-shell nuclei. The motivation comes partly from attempts to understand the fine structure of the so-called ${ }^{12} \mathrm{C}+{ }^{12} \mathrm{C}$ molecular resonances in terms of a sound, fully microscopic description. Although a fully microscopic multi-channel resonating group calculation for this challenging problem is perhaps still not quite within reach, the techniques have now been developed to a stage where it is possible to calculate all the spectroscopic amplitudes needed for the determination of the strength functions for the breakup of a specific ${ }^{12} \mathrm{C}+{ }^{12} \mathrm{C}$ quasi-bound state into various channels. The most important open channels involve ${ }^{12} \mathrm{C}+{ }^{12} \mathrm{C},{ }^{8} \mathrm{Be}+{ }^{16} \mathrm{O}, \alpha+{ }^{20} \mathrm{Ne},{ }^{23} \mathrm{Na}+\mathrm{p}$, and ${ }^{23} \mathrm{Mg}+\mathrm{n}$ structures. Spectroscopic amplitudes are needed for heavy fragments in specific excited states. In the $\alpha+{ }^{20} \mathrm{Ne}$ breakup, e.g., specific states of the $0^{+}, 2^{+}, 4^{+}, 6^{+}, 8^{+}$ground-state 
rotational band of ${ }^{20} \mathrm{Ne}$ as well as the $K=2^{-}$band with band head at $4.97 \mathrm{MeV}$ are important to an understanding of the experimentally determined strength functions ${ }^{28-33}$ ). The important channels for this problem involve two-cluster systems. For this reason further developments are made in the BS transform technique to facilitate the calculation of norm and overlap matrix elements for such cluster systems made up of two fragments. Norm and overlap matrix elements are particularly simple in the approximation in which particles in different fragments are described by oscillator functions of the same $\omega$. In this approximation norm and overlap kernels are SU(3) scalars ${ }^{34}$ ), leading to considerable simplification. For the $A=24$ system, e.g., the equal length parameter oscillator approximation should be very good. The generalization needed for the calculation of the interaction kernels and cluster systems with fragments involving oscillator functions of different size are reserved for a future study. (A detailed discussion of the interaction kernel problem for simple 3- and 4cluster systems can be found in ref. $\left.{ }^{24}\right)$ ).

The method, (outlincd in sects. 2 and 3), involves two basic steps:

(i) the calculation of the BS transform of the norm or overlap kernels and

(ii) the expansion of this transform in terms of suitably SU(3) coupled Bargmann space functions. The coefficients in these expansions give the numerical values of the norm or overlap elements. The method thus reduces the calculation of complicated multidimensional integrals to purely algebraic techniques involving the algebra of SU(3) recoupling transformations.

Two variants of the basic calculational technique have been developed. In the first, BS transforms are calculated directly for the full $A$-particle system of arbitrary space symmetry characterized by full 4-columned Young tableaux. In this method the BS transforms are expressed in terms of a few structure and exchange cocfficicnts which have to be evaluated for each case; but norm and overlap matrix elements are given in terms of one simple, universal formula. This method is particularly useful for lighter systems. It is illustrated in detail, in sect. 4 , by the $A=12$ system built from two ${ }^{6} \mathrm{Li}$ fragments which can couple to space symmetries [4422], [4431] and [444]. As a further illustration ovcrlap matrix clements with the $\alpha+{ }^{8} \mathrm{Bc}$ cluster system are also included. In heavier systems this method becomes somewhat cumbersome since the total number of structure and exchange coefficients needed for the calculation of norm and overlap matrix elements can become large. In a second variant of the general method, the BS transforms are therefore first calculated for $n$-particle subsystems of orbital symmetries characterized by single-columned Young tableaux (totally antisymmetric space symmetries). The Bargmann space transforms for the full $A$-particle system are then built from these by suitable combinations of these "single-column" functions. The calculation of the "single-column" BS transforms is quite simple. A fairly extensive compilation is given in appendix $\mathrm{C}$. The method has the disadvantage that the final form of the analytic expressions for norm and overlap matrix elements now varies from case to case since different combinations of SU(3) recoupling transformations are now needed for each specific case. 
Two specific applications are given of this technique. In the first, (sect. 5), analytic expressions are given for the norms of binary-fragment cluster systems in which a light fragment of mass number $f, f \leqq 4$, has 0 s internal excitation only. This light fragment is combined with a heavy fragment of mass number $A-f$, with $A-f \leqq 24$, where for simplicity it is assumed that the heavy fragment is in the state of highest possible space symmetry and highest possible SU(3) (oscillator quanta) symmetry. This is a useful example since the 5 cases, $4 \leqq A-f \leqq 8,8 \leqq A-f \leqq 12,12 \leqq A-f$ $\leqq 16,16 \leqq A-f \leqq 20$ and $20 \leqq A-f \leqq 24$ illustrate somewhat different problems in the recoupling technique.

A second application, (sect. 6), gives the spectroscopic amplitudes for the most important open channels for the ${ }^{12} \mathrm{C}+{ }^{12} \mathrm{C}$ "molecular resonances". Under the assumption that the most important fine structure components of these resonances are related to "molecular states", the ${ }^{12} \mathrm{C}+{ }^{12} \mathrm{C}$ channels are restricted to the ${ }^{12} \mathrm{C}$ bands of SU(3) symmetry $(\lambda \mu)=(04)$. That is, the excitation of each ${ }^{12} \mathrm{C}$ nucleus is restricted to the $0^{+}, 2^{+}, 4^{+},(\lambda \mu)=(04)$ rotational band of ${ }^{12} \mathrm{C}$. The other channels to be included are ${ }^{16} \mathrm{O}+{ }^{8} \mathrm{Be}\left(0^{+}, 2^{+}, 4^{+}\right), \alpha+{ }^{20} \mathrm{Ne}$, including the states of the ground-state $K=0^{+}$band of ${ }^{20} \mathrm{Ne}$ in the $\mathrm{SU}(3)$ approximation $(\lambda \mu)=(80)$, and the $K=2^{-}$band of ${ }^{20} \mathrm{Ne}$, (band head at $4.97 \mathrm{MeV}$ ) which should be approximated extremely well by the SU(3) quantum numbers $(\lambda \mu)=(82)$; and finally, the ${ }^{23} \mathrm{Na}+\mathrm{p}$ and ${ }^{23} \mathrm{Mg}+\mathrm{n}$ channels where the excited states of the $A=23$ nuclei are approximated as members of $(\lambda \mu)=(83)$ rotational bands.

To facilitate the discussion, a number of useful properties of SU(3) recoupling coefficients are collected for easy reference in appendix A. (Some of the notation pertaining to $\mathrm{SU}(3)$ is carefully defined in this appendix.) To keep the algebraic manipulations of SU(3) coupled Bargmann space functions to a minimum a number of subsidiary formulae involving these functions are collected in appendix $B$ together with a few sample derivations of such formulae.

\section{Formulation of the problem; the ${ }^{12} \mathrm{C}+{ }^{12} \mathrm{C}$ resonances}

In resonating group calculations, in general, and in the ${ }^{12} \mathrm{C}+{ }^{12} \mathrm{C}$ problem, in particular, all state vectors are expanded in properly antisymmetrized cluster functions with different fragment decompositions in different channels.

\subsection{THE SU(3) COUPLED CLUSTER BASIS}

To calculate matrix elements in a cluster function basis by the combination of BS integral transform and SU(3) recoupling techniques, it is important to expand the cluster functions in an SU(3) coupled oscillator basis. For a 2-cluster function, e.g., made up of fragments of mass numbers $f$ and $A-f$ the wave functions are to be expanded in

$$
\mathscr{A}\left[\left[\phi\left(\xi_{f}\right)^{\left(\lambda_{f} \mu_{r}\right)} \phi\left(\xi_{A-f}\right)^{\left(\lambda_{A}-f \mu_{A-r}\right)}\right]^{\left(\lambda_{c} \mu_{c}\right) \rho} \chi(R)^{(Q 0)}\right]_{a}^{\left(\lambda_{\mu)}\right)}
$$


Here, $\mathscr{A}$ is the antisymmetrization operator, $\mathscr{A}=(A)^{-1} \sum(-1)^{\sigma(P)} P$, where the sum extends over all permutations $P$. The internal wave functions, $\phi$, of the two fragments are assumed to have good SU(3) (oscillator quanta) symmetry, given by the Elliott $\left.{ }^{35}\right)$ quantum numbers $\left(\lambda_{f} \mu_{f}\right)$ and $\left(\lambda_{A-f} \mu_{A-f}\right)$. The $\phi$ are functions of $f-1$ and $A-f-1$ intcrnal coordinate vectors and include the full spin and isospin dependence of the cluster functions. They are assumed to have good SU(4) and hence good space symmetry, but the SU(4) quantum numbers are suppressed. To be more precise, for economy of notation, it will be assumed that each fragment spin-isospin function is an SU(4) scalar with $S_{f}=T_{f}=0, S_{A-f}=T_{A-f}=0$, as in the ${ }^{12} \mathrm{C}+{ }^{12} \mathrm{C}$ cluster system. The square brackets denote $\mathrm{SU}(3)$ coupling. (If the fragment spin-isospin functions are not SU(4) scalars, the square bracket is interpreted to include the SU(4) coupling of the internal functions, $\phi)$. If the product $\left(\lambda_{s} \mu_{f}\right) \times\left(\lambda_{A-f} \mu_{A-f}\right)$ is not free of outer multiplicity, a multiplicity label $\rho$ must be included with $\left(\lambda_{c} \mu_{c}\right)$ (see appendix A). The resultant coupled function of $\mathrm{SU}(3)$ symmetry $(\lambda \mu)$ is characterized by the subgroup labels $\alpha$. These can be chosen in any convenient basis, e.g. the $\kappa J M_{J}$ basis ${ }^{36}$ ) which includes the angular momentum quantum numbers $J M_{J}$; or a cartesian oscillator basis, or the closely related $U(1) \times S U(2)$ subgroup basis, labeled by the Elliott intrinsic quantum numbers $\varepsilon \Lambda M_{A}$. The relative motion function is an oscillator function in the relative coordinate vector

$\boldsymbol{R}=-\left[\frac{A-f}{A}\right]^{\frac{1}{2}}\left(\frac{1}{f^{\frac{1}{2}}}\left[\boldsymbol{r}_{1}+\ldots+\boldsymbol{r}_{f}\right]\right)+\left[\frac{f}{A}\right]^{\frac{1}{2}}\left(\frac{1}{[A-f]^{\frac{1}{2}}}\left[\boldsymbol{r}_{f+1}+\ldots+\boldsymbol{r}_{A}\right]\right)$,

where the single-particle position vectors, and hence $\boldsymbol{R}$, are dimensionless variables. Each dimensionless $\boldsymbol{r}_{i}$ is equal to the physical single-particle coordinate vector (measured in $\mathrm{cm}$ or $\mathrm{fm}$ ) divided by $[\hbar / m \omega]^{\frac{t}{3}}$. The equal length parameter approximation is made; that is the same $\omega$ is used for particles in fragments $f$ and $A-f$.

A basis, in which the fragment spins, $I_{f}, I_{A-f}$, channel spins $I_{c}$, and orbital angular momenta $L$, are good quantum numbers, can be expanded in these SU(3)-coupled cluster functions by means of $S U(3) \supset R(3)$ reduced (double-barred) Wigner coefficients ${ }^{36}$ )

$$
\begin{aligned}
\mathscr{A}\left(\left(\phi_{\kappa_{f} I_{f}}^{\left(\lambda_{f} \mu_{f}\right)} \times\right.\right. & \left.\left.\phi_{\kappa_{A}-f I_{A}-f}^{\left(\lambda_{A}-f \mu_{A}\right)}\right)_{I_{c}} \times \chi_{L}^{(Q 0)}\right)_{J M_{J}} \\
& =\sum_{\left(\lambda_{c} \mu_{c}\right) \kappa_{c}} \sum_{(\lambda \mu) \kappa}\left\langle\left(\lambda_{f} \mu_{f}\right) \kappa_{f} I_{f} ;\left(\lambda_{A-f} \mu_{A-f}\right) \kappa_{A-f} I_{A-f} \|\left(\lambda_{c} \mu_{c}\right) \kappa_{c} I_{c}\right\rangle_{\rho} \\
& \times\left\langle\left(\lambda_{c} \mu_{c}\right) \kappa_{c} I_{c} ;(Q 0) L \|(\lambda \mu) \kappa J\right\rangle \\
& \times \mathscr{A}\left[\left[\phi^{\left(\lambda_{f} \mu_{f}\right)} \times \phi^{\left(\lambda_{A}-\mu_{A}-f\right)}\right]^{\left(\lambda_{c} \mu_{c}\right) \rho} \times \chi^{(Q 0)}\right]_{\kappa J M}^{\left(\lambda_{j} \mu\right)} .
\end{aligned}
$$

The round brackets now denote ordinary angular momentum coupling. (Fragment spin-isospin functions are again assumed to be SU(4) scalars.) The SU(3) coupled form, eq. (1) has a number of important advantages.

(i) The complications associated with the details of the angular momentum cou- 
pling can be avoided till the very last step of a calculation. Matrix elements in the SU(3) coupled basis can be expressed solely in terms of (subgroup label-independent) Racah or recoupling coefficients. Dependence on fragment and channel spins can be introduced in the last step of a calculation by means of eq. (3).

(ii) Norm and overlap matrix elements for several channels are handled at once in the SU(3) coupled basis. All members of SU(3) bands of $\left(\lambda_{f} \mu_{f}\right)$ and $\left(\lambda_{A-f} \mu_{A-f}\right)$ are included in one common formula.

(iii) Since the $\mathscr{A}$ operator is an SU(3) scalar, its matrix elements are diagonal in $(\lambda \mu)$, and the eigenfunctions of the norm and overlap kernels are the SU(3) coupled functions of eq. (1) or linear combinations of such functions with the same final $(\lambda \mu)$. This important point was first stressed by Horiuchi ${ }^{34}$ ) and is very useful in identifying the Pauli-forbidden components of the cluster basis. This point can be illustrated by the ${ }^{12} \mathrm{C}+{ }^{12} \mathrm{C}$ cluster decomposition of the $A=24$ system. With ${ }^{12} \mathrm{C}$ internal functions of SU(3) symmetry $\left(\lambda_{f} \mu_{f}\right)=(04),\left(\lambda_{A-f} \mu_{A-f}\right)=(04)$, the possible channel internal SU(3) quantum numbers are $\left(\lambda_{c} \mu_{c}\right)=(08),(16),(24),(32)$ and $(40)$. For such a system of two identical fragments, however, the symmetrically coupled internal functions with $\lambda_{c}+\mu_{c}=$ even, $\left(\lambda_{c} \mu_{c}\right)=(08),(24),(40)$, can couple only with relative motion functions of even $Q$, (and hence even $L$ ), and have positive parity; whereas the antisymmetrically coupled internal functions with $\lambda_{c}+\mu_{c}=$ odd, $\left(\lambda_{c} \mu_{c}\right)=(16)$, (32) couple only with relative motion functions of odd $Q$ and have negative parity. (The $\mathscr{A}$ operator destroys functions with $\lambda_{c}+\mu_{c}+Q=$ odd.) The dominant components of the quasi-bound sub-Coulomb resonant states of this system can be expected to have large overlaps with shell-model states of $2 \hbar \omega$ oscillator excitation. Hence cluster states with $Q=14=[Q($ minimum Pauli-allowed $)+2]$ are of particular interest. The possible $\left(\lambda_{c} \mu_{c}\right),(\lambda \mu)$ combinations of such states are listed in table 1. There are 14 possible $0^{+}$states in this space. In an $\left(\left(I_{1} I_{2}\right) I_{c} L\right) 0^{+}$angular-momentum coupled basis these correspond to the $\left(I_{1} I_{2}\right)$ combinations: $(00),(22),(44)$ with $I_{c}=0 ;(02),(24),(22),(44)$ with $I_{c}=2 ;(04),(24),(22)$, (44) with $I_{c}=4 ;$ (24), (44) with $I_{c}=6$; and (44) $I_{c}=8$; all with $I_{c}=L$, where only symmetrically coupled $\left(I_{1} I_{2}\right)$

TABle 1

Possible SU(3) quantum numbers for the ${ }^{12} \mathrm{C}+{ }^{12} \mathrm{C}$ cluster functions with $Q=14$, (2hw oscillator excitations)

\begin{tabular}{|c|c|c|c|c|c|c|c|c|c|c|c|}
\hline$\left(\lambda_{c} \mu_{c}\right)$ & \multicolumn{11}{|c|}{$(\lambda \mu)$} \\
\hline (08) & $\begin{array}{l}(14,8) \\
(13,7)\end{array}$ & & $(12,6)$ & $(11,5)$ & & & $(10,4)$ & (93) & (82) & $\begin{array}{l}(71) \\
(60)\end{array}$ & \\
\hline (24) & $\begin{array}{l}(16,4) \\
(14,5)\end{array}$ & $\begin{array}{l}(15,3) \\
(13,4)\end{array}$ & $(12,6)$ & $(11,5)$ & $(14,2)$ & $(12,3)$ & $(10,4)$ & (93) & (82) & $\begin{array}{l}(13,1) \\
(12,0)\end{array}$ & $\begin{array}{l}(11,2) \\
(10,1)\end{array}$ \\
\hline$(40)$ & $(18,0)$ & $(16,1)$ & & & $(14,2)$ & $(12,3)$ & $(10,4)$ & & & & \\
\hline
\end{tabular}


are possible. Seven of the $140^{+}$states are completely Pauli forbidden. The norm matrix has 7 zero eigenvalues. In the $\left(\left(I_{1} I_{2}\right) I_{c} L\right) 0^{+}$basis, however, the determination of the 7 Pauli-forbidden vectors involves the diagonalization of a $14 \times 14$ matrix. In the $\mathrm{SU}(3)$ coupled basis states with $J^{\pi}=0^{+}$occur only in representations with $\lambda=$ even, $\mu=$ even. Of these, the five $0^{+}$states with $(\lambda \mu)=(18,0),(16,4),(14,8),(12,6)^{2}$ can be identified at once as completely Pauli-forbidden since the $A=24$ shell-model space of $2 \hbar \omega$ oscillator excitation has no states of these high SU(3) symmetries. In addition, it can be seen that there is only one $2 \hbar \omega$ shell-model excitation of SU(3) symmetry $(14,2)$ in the $A=24$ nucleus, the shell-model state $\left|\mathrm{s}^{4} \mathrm{p}^{12}\left[\mathrm{sd}^{6}(82) \mathrm{pf}^{2}(60)\right](14,2) 0^{+}\right\rangle$. One linear combination of states with $\left(\lambda_{c} \mu_{c}\right)=(24),(40)$ and $(\lambda \mu)=(14,2)$ must therefore be Pauli forbidden. The identification of this Pauli-forbidden state, however, is now part of a 2-dimensional (rather than a 14-dimensional) eigenvalue problem. The 7 Pauli-allowed states with $Q=14$ and $J^{\pi}=0^{+}$have $\mathrm{SU}(3)$ quantum numbers $(14,2),(12,0),(10,4)^{2},(82)^{2},(60)$. Of the 40 possible states with $Q=14, J^{\pi}=2^{+}, 20$ are completely Pauli forbidden; and of the 57 states with $Q=14, J^{\pi}=4^{+}, 31$ are completely Pauli forbidden.

\subsection{THE ${ }^{12} \mathrm{C}+{ }^{12} \mathrm{C}$ RESONANCES}

Much of the motivation for the development of the techniques of this investigation comes from an attempt to understand the socalled molecular resonances ${ }^{37}$ ) in the ${ }^{12} \mathrm{C}+{ }^{12} \mathrm{C}$ reactions in terms of such a microscopic description of this system. Although a large number of theoretical studies of these resonances seems to confirm the usefulness of the quasi-molecular picture, an interpretation of the gross structure behavior of these resonances may be possible without invoking a model of molecular pocket resonances ${ }^{38-40}$ ). Evidence for the nuclear molecular picture must thus be sought in the fine structure of these resonances. The large number of closely spaced, narrow fine structure components are the distinctive feature of the ${ }^{12} \mathrm{C}+{ }^{12} \mathrm{C}$ resonances. A simpler fine structure is observed in the ${ }^{16} \mathrm{O}+{ }^{16} \mathrm{O}$ resonances, for example. In the language of the SU(3) coupled cluster basis, this may be related to the much richer number of possible $\left(\lambda_{c} \mu_{c}\right),(\lambda \mu)$ values in the ${ }^{12} \mathrm{C}+{ }^{12} \mathrm{C}$ system. Most recent theoretical interpretations make use of some variant of the Imanishi-Nogami model ${ }^{41}$ ), based on an internal excitation of the ${ }^{12} \mathrm{C}$ nuclei ${ }^{42-44}$ ). The band crossing model of Kondō et al. ${ }^{45}$ ), in particular, reproduces some of the fine structure observed in particular exit channels. As recently pointed out by Berger and Gogny, none of the theoretical models account for all of the key features of these resonances ${ }^{46}$ ), and none of the models are sufficiently microscopic to be capable of examining the large amount of recent experimental information which is now furnishing strength functions to many different exit channels.

Although a fully microscopic multi-channel resonating group calculation for this challenging problem is perhaps not yet within reach, it may be possible to gain some 
understanding of the resonance fine structure in terms of predicted spectroscopic amplitudes for the various observed exit channels. Since specific exit channels often exhibit markedly different strength factors for different fine structure components of the excitation functions, it may be possible to gain an understanding of the structure of specific quasi-bound states of the ${ }^{12} \mathrm{C}+{ }^{12} \mathrm{C}$ system from a knowledge of such spectroscopic amplitudes.

For the closely spaced narrow resonances of the ${ }^{12} \mathrm{C}+{ }^{12} \mathrm{C}$ reactions we assume that the $T$-matrix can be approximated in terms of a few partial width amplitudes $\Gamma_{v c}^{\frac{1}{2}}$ by

$$
T_{c c^{\prime}}=\exp \left(i \delta_{c}+i \delta_{c^{\prime}}\right) \sum_{v} \frac{\Gamma_{v c}^{\frac{1}{2}} \Gamma_{v}^{\frac{1}{2} c^{\prime}}}{E-\varepsilon_{v}}
$$

where the entrance channel $c$ consists of ${ }^{12} \mathrm{C}+{ }^{12} \mathrm{C}$ with $I_{f}=I_{A-f}=0$. The exit channels $c^{\prime}$ to be included are:

(i) ${ }^{12} \mathrm{C}+{ }^{12} \mathrm{C}$ with $I_{f}=0,2,4 ; I_{A-f}=0,2,4$. The ${ }^{12} \mathrm{C}$ nuclei can be in states of their internal rotational excitations, that is in their ground-state rotational bands with $\left(\lambda_{f} \mu_{f}\right)=(04)$;

(ii) $\alpha+{ }^{20} \mathrm{Ne}$ with $I_{\mathrm{Ne}}^{\pi}=0^{+}, 2^{+}, 4^{+}, 6^{+}, 8^{+}$, with the ${ }^{20} \mathrm{Ne}$ excitations in the groundstate $K=0^{+}$band described in the $\operatorname{SU}(3)$ approximation $\left(\lambda_{A-f^{\prime}} \mu_{A-f^{\prime}}\right)=(80)$; or with $I_{\mathrm{Ne}}^{n}=2^{-}, 3^{-}, \ldots$, again within the SU(3) approximation which describes these members of this $K=2^{-}$band in terms of a pure $\left(\lambda_{A-f^{\prime}} \mu_{A-f^{\prime}}\right)=(82)$;

(iii) ${ }^{16} \mathrm{O}+{ }^{8} \mathrm{Be}$ with $I_{\mathrm{Be}}^{\pi}=0^{+}, 2^{+}, 4^{+} ;\left(\lambda_{f^{\prime}} \mu_{f^{\prime}}\right)=(40)$;

(iv) ${ }^{23} \mathrm{Na}+\mathrm{p}$ and ${ }^{23} \mathrm{Mg}+\mathrm{n}$, with $I^{\pi}=\frac{3}{2}^{+}, \frac{5}{2}^{+}, \frac{7}{2}^{+}, \ldots$, and $\frac{1}{2}^{+}, \frac{3}{2}^{+}, \frac{5}{2}^{+}, \ldots$; where these rotational bands are approximated as members of pure $\left(\lambda_{A-f^{\prime}} \mu_{A-f^{\prime}}\right)=(83)$ $\kappa_{J}=\frac{3}{2}$ and $\frac{1}{2}$ bands.

The quasi-bound states $|v\rangle$ are to be built from the states $|i\rangle$ which have the $\mathrm{SU}(3)$ quantum numbers of the ${ }^{12} \mathrm{C}+{ }^{12} \mathrm{C}$ molecular states

$$
\mathscr{A}\left|\left[\left[\phi\left({ }^{12} \mathrm{C}\right)^{(04)} \times \phi\left({ }^{12} \mathrm{C}\right)^{(04)}\right]^{\left(\lambda_{c} \mu_{c}\right)} \times \chi(R)^{(Q 0)}\right]_{\kappa J M J}^{\left(\lambda_{\mu}\right)}\right\rangle,
$$

with $\kappa=\mu, \mu-2, \ldots, 0$ or 1 . Although all calculations are carried out as algebraic functions of $Q$ so that ${ }^{12} \mathrm{C}+{ }^{12} \mathrm{C}$ relative motion functions of complicated shape can be accommodated, the dominant components of the quasi-bound states can be expected to have large overlaps with shell-model states of $2 \hbar \omega$ oscillator excitation. These are the states with $Q=14$ enumerated in table 1 . In first approximation therefore the basis set for the quasi-bound states for the sub-Coulomb resonances is chosen to be the set $|i\rangle$ consisting of the Pauli-allowed (normalized) eigenvectors of the states (5), with $Q=14$. The expansion coefficients, $\left\langle\left(\lambda_{c} \mu_{c}\right)(\lambda \mu) \mid i\right\rangle$, which give the eigenvectors of the operator, $1 \mathscr{A}$, in the ${ }^{12} \mathrm{C}+{ }^{12} \mathrm{C}$ cluster basis, are calculated in sect. 6 .

Since the observed resonances of the same $J^{\pi}$ value fall within an energy interval of $\sim 2 \mathrm{MeV}$, and since individual fine structure components may correspond to quasi-bound states of very similar structure, the penetrability factors associated with 
the partial width amplitudes $\left(\Gamma_{v c}^{\frac{1}{2}} \Gamma_{v c}^{\frac{1}{2}}\right)$ can be expected to be approximately independent of $v$. The relative magnitudes of the $\left(\Gamma_{v c^{\prime}}^{\frac{1}{2}} \Gamma_{v c}^{\frac{t}{t}}\right)$ for different $v$ 's can thus be expected to be proportional to the relative magnitudes of the corresponding spectroscopic amplitudes $\left(A_{v c^{\prime}} A_{v c}\right)$, and it may be possible to gain an understanding of the experimentally determined strength functions in terms of the spectroscopic amplitudes, $A_{v c}=\langle v \mid c\rangle$. These can be calculated in terms of overlap matrix elements of the type to be discussed in this work.

Some calculations of ${ }^{12} \mathrm{C}+{ }^{12} \mathrm{C}$ norm kernels and overlap kernels with the ${ }^{16} \mathrm{O}+$ ${ }^{8} \mathrm{Be}$ and ${ }^{20} \mathrm{Ne}+\alpha$ channels have previously been made by Tohsaki-Suzuki ${ }^{47}$ ). Results are given in a coordinate representation. The emphasis in the present investigation is on spectroscopic amplitudes for specific ${ }^{12} \mathrm{C}+{ }^{12} \mathrm{C}$ molecular states, $|i\rangle$ and the many open exit channels $\left|c^{\prime}\right\rangle$. Such amplitudes can be calculated in terms of the overlap matrix elements evaluated in sect. 6 . The solution of the quasi-bound state eigenvalue problem and the details of the coupling of the quasi-bound states to the continuum states will be left to a subsequent investigation. In the present work, the emphasis is on development of the techniques necessary for calculating the needed norm and overlap matrix elements.

\section{Formulation of the technique}

\subsection{OVERLAP MATRIX ELEMENTS AND RESONATING GROUP KERNELS}

In the SU(3) coupled basis of eq. (1), resonating group kernels and spectroscopic amplitudes of the type discussed above can all be evaluated in terms of matrix elements of the following general type

$$
\begin{aligned}
\left\langle\left[\left[\phi^{\left(\lambda_{f} \mu_{f}\right)} \times \phi^{\left(\lambda_{A}-f_{A}-f\right)}\right]^{\left(\lambda_{c} \mu_{c}\right)} \times \chi(\overline{\boldsymbol{R}})^{(Q 0)}\right]_{\alpha}^{(\lambda \mu)} \chi\left(\boldsymbol{R}_{\mathrm{c} . \mathbf{m}}\right)^{(00)}\right| \mathcal{O A} \\
\quad \times\left|\left[\left[\phi^{\left(\lambda_{f^{\prime}} \mu_{\left.f^{\prime}\right)}\right)} \times \phi^{\left(\lambda_{A}-f^{\prime} \mu_{A}-f^{\prime}\right)}\right]^{\left(\lambda_{c}^{\prime} \mu_{c}^{\prime}\right)} \times \chi(\boldsymbol{R})^{\left(Q^{\prime} 0\right)}\right]_{\alpha^{\prime \prime}}^{\left(\lambda^{\prime \prime} \mu^{\prime \prime}\right)} \chi\left(\boldsymbol{R}_{\mathrm{c} . \mathrm{m}}\right)^{(00)}\right\rangle,
\end{aligned}
$$

where $\mathcal{O}=H$ (a hamiltonian), or $\mathcal{O}=1$ (the unit operator). The Dirac bracket notation implies integration over all spatial coordinates and a summation over all spin and isospin coordinates. A c.m. oscillator function with $0 \hbar \omega$ excitation, [SU(3) quantum numbers $(00)]$, has been added so that the spatial integration can be carried out over all single-particle coordinates $\boldsymbol{r}_{i}, i=1, \ldots, \boldsymbol{A}$ on an equal footing. $\overline{\boldsymbol{R}}$ and $\boldsymbol{R}$ are functions of $\boldsymbol{r}_{i}$ given by eq. (2), with cluster decomposition $f, A-f$ for $\bar{R}$, and $f^{\prime}, \boldsymbol{A}-\boldsymbol{f}^{\prime}$ for $\boldsymbol{R}$.

With the use of $\mathrm{SU}(3)$ coupled functions it is natural to expand the relative motion functions in an oscillator basis. It may, however, be useful to relate the matrix elements (6) to the usual norm and interaction kernels which are part of the standard notation of resonating group calculations. (For economy of notation, let us for this subsection assume that $\left(\lambda_{f} \mu_{f}\right)-\left(\lambda_{A-} \mu_{A-f}\right)=(00)$ and that $\left.f^{\prime}=f\right)$. A resonating group function can then be written in terms of the cluster functions

$$
\psi=\mathscr{A} \phi_{f} \phi_{A-f} F(\boldsymbol{R}) \chi\left(\boldsymbol{R}_{\mathrm{c} . \mathrm{m} .}\right)
$$


The relative motion function, $F(\boldsymbol{R})$, of arbitrary shape can be expanded in $\delta$-functions in terms of the parameter coordinate ${ }^{1}$ ), $\boldsymbol{R}^{\prime}$,

$$
F(R)=\int \mathrm{d} \boldsymbol{R}^{\prime} \delta\left(\boldsymbol{R}-\boldsymbol{R}^{\prime}\right) F\left(\boldsymbol{R}^{\prime}\right),
$$

to yield

$$
\langle\psi|\mathscr{O} \mathscr{A}| \psi\rangle=\iint \mathrm{d} \boldsymbol{R}^{\prime \prime} \mathrm{d} \boldsymbol{R}^{\prime} F\left(\boldsymbol{R}^{\prime \prime}\right)^{*} \mathscr{K}\left(\boldsymbol{R}^{\prime \prime}, \boldsymbol{R}^{\prime}\right) F\left(\boldsymbol{R}^{\prime}\right),
$$

where the kernels, $\mathscr{K}\left(\boldsymbol{R}^{\prime \prime}, \boldsymbol{R}^{\prime}\right)$, are defined by

$$
\mathscr{K}\left(\boldsymbol{R}^{\prime \prime}, \boldsymbol{R}^{\prime}\right)=\left\langle\phi_{f} \phi_{A-f} \delta\left(\boldsymbol{R}-\boldsymbol{R}^{\prime \prime}\right) \chi\left(\boldsymbol{R}_{\text {c.m. }}\right)|\mathcal{O A}| \phi_{f} \phi_{A-f} \delta\left(\boldsymbol{R}-\boldsymbol{R}^{\prime}\right) \chi\left(\boldsymbol{R}_{\text {c.m. }}\right)\right\rangle .
$$

An expansion of $F(\boldsymbol{R})$ in oscillator functions $\chi(R)_{L M}^{(Q 0)}$ leads to the matrix elements

$$
\begin{aligned}
\iint \mathrm{d} \boldsymbol{R}^{\prime \prime} \mathrm{d} \boldsymbol{R}^{\prime} \chi\left(\boldsymbol{R}^{\prime \prime}\right)_{L M}^{(\mathcal{Q} O) *} \mathscr{K}\left(\boldsymbol{R}^{\prime \prime}, \boldsymbol{R}^{\prime}\right) \chi\left(\boldsymbol{R}^{\prime}\right)_{L^{\prime} M^{\prime}}^{\left(Q^{\prime}\right)} \\
=\left\langle\phi_{f} \phi_{A-f} \chi(\boldsymbol{R})_{L M}^{(Q 0)} \chi\left(\boldsymbol{R}_{\mathrm{c} . \mathrm{m} .}\right)^{(00)}|\mathcal{O} \mathscr{A}| \phi_{f} \phi_{A-f} \chi(\boldsymbol{R})_{\left.L^{\prime} M^{\prime}\right)}^{\left(Q^{\prime}\right)} \chi\left(\boldsymbol{R}_{\mathrm{c} . \mathrm{m} .}\right)\right\rangle,
\end{aligned}
$$

which are of the type of eq. (6). If $\mathcal{O}$ is an $\mathrm{SU}(3)$ scalar operator, (e.g. $\mathcal{O}=1)$, then

$$
\mathscr{K}\left(\boldsymbol{R}^{\prime \prime}, \boldsymbol{R}^{\prime}\right)=\sum_{Q} \Lambda_{Q} \sum_{L} \mathscr{R}\left(\boldsymbol{R}^{\prime \prime}\right)_{L}^{(Q 0)} \mathscr{R}\left(\boldsymbol{R}^{\prime}\right)_{L}^{(Q 0)} \frac{2 L+1}{4 \pi} \boldsymbol{P}_{L}\left(\hat{\boldsymbol{R}}^{\prime \prime} \cdot \hat{\boldsymbol{R}}^{\prime}\right) .
$$

Here, $\Lambda_{Q}$ is the value of the matrix element $(9 \mathrm{a})$, with $Q^{\prime}=Q, L M^{\prime}=L M$. [Note that this is independent of $L, M$ when $\mathcal{O}$ is an SU(3) scalar operator.] The $\mathscr{R}\left(R^{\prime \prime}\right)_{L}^{(\mathcal{Q 0 )}}$ are normalized radial harmonic oscillator functions, with $Q=2 N+L$; and $P_{L}(\cos \alpha)$ is a Legendre polynomial of the angle between the unit vectors $\hat{R}^{\prime \prime}, \hat{\boldsymbol{R}}^{\prime}$. The generalization of eq. (9b) for fragments with internal functions $\left[\phi^{\left(\lambda_{f} \mu_{f}\right)} \times \phi^{\left(\lambda_{A}-f \mu_{A}-f\right)}\right]_{\kappa_{c} I_{c} M_{c}}^{\left(\lambda_{c} \mu_{c}\right)}$ is given in sect. 6 , see eqs. (109a-b).

\subsection{THE BARGMANN-SEGAL TRANSFORM}

The calculation of the complicated multi-dimensional integrals implied by eq. (6) can be reduced to a purely algebraic problem by the introduction of the BS transform ${ }^{22-24}$ ) of the operators $\mathcal{O A}$. For this purpose, however, it is advantageous to separate the spin-isospin and orbital integrations by standard techniques. The SU(4) symmetries of the various cluster fragments can be coupled by standard unitary group technology 48,49 ), and the problem can be reduced to one of evaluating orbital integrals. In the orbital integrals the antisymmetrizer, $\mathscr{A}$, must be replaced by a Young operator, $Y$, built from products of symmetrizers, determined by the row structure of the Young tableaux, and antisymmetrizers, determincd by the column structurc of the tableaux which characterize the full space symmetry of the $A$-particle functions. Either the symmetric or antisymmetric form ${ }^{50}$ ) of $Y$ can be used since the details of 
the subgroup labeling of the permutation group are unimportant for our purposes. The BS transform of the operator $\mathcal{O} Y$ can then be introduced by

$$
H\left(\bar{k}, \boldsymbol{k}^{*}\right)=\int \mathrm{d} \boldsymbol{r}_{1} \ldots \int \mathrm{d} \boldsymbol{r}_{A} \prod_{i=1}^{A} A\left(\overline{\boldsymbol{k}}_{i}, \boldsymbol{r}_{i}\right) \mathcal{O Y} \prod_{i=1}^{A} A\left(\boldsymbol{k}_{i}^{*}, \boldsymbol{r}_{i}\right) .
$$

The $A\left(\bar{k}_{i}, \boldsymbol{r}_{i}\right)$, [and $A\left(\boldsymbol{k}_{i}, \boldsymbol{r}_{i}\right)$, are the kernel functions for the BS transform, here initially expressed in single-particle coordinates,

$$
A\left(\boldsymbol{k}_{i}, \boldsymbol{r}_{i}\right)=\prod_{\alpha=x, y, z} A\left(k_{i_{\alpha}}, x_{i_{\alpha}}\right)
$$

where

$$
A\left(k_{x}, x\right)=\pi^{-\frac{1}{4}} \exp \left\{-\frac{1}{2} k_{x}^{2}-\frac{1}{2} x^{2}+\sqrt{2} k_{x} x\right\} .
$$

The 1-dimensional BS transform is a unitary integral transform from $\mathscr{L}^{2}(\mathbb{R})$ to a space of square integrable analytic functions with the measure

$$
\mathrm{d} g\left(k_{x}\right)=\pi^{-1} \mathrm{e}^{-k_{x} k_{x}^{*}} \mathrm{~d} \operatorname{Re}\left(k_{x}\right) \mathrm{d} \operatorname{Im}\left(k_{x}\right),
$$

in the complex $k_{x}$ space; with

$$
F\left(k_{x}\right)=\int_{-\infty}^{\infty} \mathrm{d} x A\left(k_{x}, x\right) \psi(x),
$$

and inverse

$$
\psi(x)=\int \mathrm{d} g\left(k_{x}\right) A^{*}\left(k_{x}, x\right) F\left(k_{x}\right)
$$

In the present application, the harmonic oscillator generating function property of $A$ is of prime importance

$$
A\left(k_{x}, x\right)=\sum_{n=0}^{\infty} \chi_{n}(x)^{*} k_{x}^{n} / \sqrt{ } n !
$$

where $\chi_{n}(x)$ is a normalized 1-dimensional harmonic oscillator function, and $k_{x}^{n} / \sqrt{n !}$ is the corresponding Bargmann space transform. The 3-dimensional Bargmann space function,

$$
P(\boldsymbol{k})_{\alpha}^{(Q 0)}=\frac{k_{x}^{n_{x}}}{\sqrt{ } n_{x} !} \frac{k_{y}^{n_{y}}}{\sqrt{ } n_{y} !} \frac{k_{z}^{n_{z}}}{\sqrt{ } n_{z} !},
$$

is the BS transform of the 3-dimensional oscillator function $\chi(r)_{\alpha}^{(Q 0)}$. It has SU(3) irreducible tensor character $(Q 0)$ with $Q=n_{x}+n_{y}+n_{z}$, and subgroup labels, $\alpha=$ $n_{x} n_{y} n_{z}$, here given in a cartesian oscillator basis. Note that in this case $n_{x} n_{y} n_{z}$ are equivalent to the Elliott intrinsic labels with $\varepsilon=2 n_{z}-n_{x}-n_{y}, M_{A}=\frac{1}{2}\left(n_{x}-n_{y}\right)$, since $\Lambda=\frac{1}{6}(2 Q-\varepsilon)$ is a redundant label with $(\lambda \mu)=(Q 0)$, i.e. with $\mu=0$. A linear combination of (16) gives the Bargmann space polynomials $P_{L M}^{(Q)}$ in an angular momentum 
basis, ( $\alpha=L M, \kappa=$ redundant quantum number in this case). The corresponding function of $k^{*}$ has $\mathrm{SU}(3)$ irreducible character $(0 Q)$

$$
P\left(k^{*}\right)_{\alpha}^{(0 Q)}=\frac{k_{x}^{* n_{x}}}{\sqrt{n_{x} !}} \frac{k_{y}^{* n_{y}}}{\sqrt{n_{y} !}} \frac{k_{z}^{* n_{z}}}{\sqrt{n_{z} !}}=P\left(k^{*}\right)_{-\varepsilon A-M_{A}}^{(0 Q)}(-1)^{\ddagger Q-\frac{1}{z} \varepsilon+M_{A}},
$$

where the phase factor associated with complex conjugation ${ }^{36}$ ) is absorbed in the definition of the subgroup label $\tilde{\alpha}$.

In single-particle coordinates the BS transforms of the operators can be evaluated by straightforward integrations. For $\mathcal{O}=1$, and $Y=\sum_{P} c_{P} P$,

$$
H\left(\bar{k}, \boldsymbol{k}^{*}\right)=\sum_{P} c_{P} \exp \left\{\sum_{i=1}^{A}\left(\overline{\boldsymbol{k}}_{\boldsymbol{i}} \cdot P \boldsymbol{k}_{i}^{*}\right)\right\}
$$

where, for the transposition $P=(12)$, e.g.: $P k_{1}^{*}=k_{2}^{*}, P k_{2}^{*}=k_{1}^{*}$. Implicitly, all oscillator matrix elements are contained in such transforms. Specific matrix elements can be extracted by expansions of the exponentials or by differentiations with respect to the $k_{i \alpha}, k_{j \beta}^{*}$ variables, making use of the property

$$
\frac{1}{\sqrt{ } n !}\left[\frac{\partial^{n}}{\partial \bar{k}_{i_{\alpha}}^{n}} A\left(k_{i_{\alpha}}, x_{i_{\alpha}}\right)\right]_{\bar{k}_{i_{\alpha}}=0}=\chi_{n}\left(x_{i_{\alpha}}\right)^{*} .
$$

To gain the integrals needed for a nuclear cluster or reaction problem, it is necessary to introduce cluster internal and relative coordinate vectors. For a binary cluster decomposition into fragments of masses $f$ and $A-f$ such coordinates can be intro-
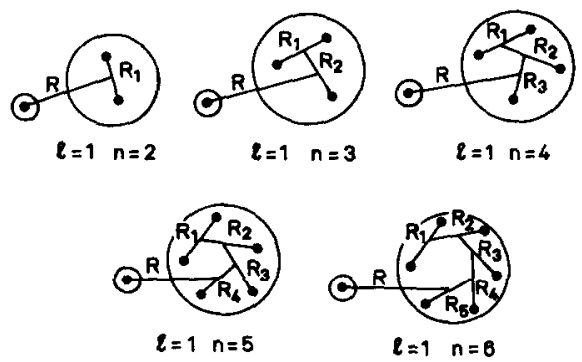

(a)

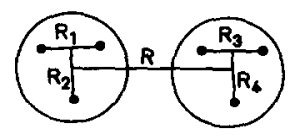

$t=3 \quad n=3$

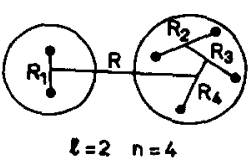

(b)

Fig. 1. Cluster coordinates for binary fragment systems; (a) $(1+n)$-particle systems, (b) $(l+n)$-particle systems. Fragment internal coordinates are denoted by a subscript, $\boldsymbol{R}_{1}, \boldsymbol{R}_{2}, \ldots$, the coordinate $\boldsymbol{R}$ (without subscript) is the relative coordinate vector of the two fragments. 
duced by the orthogonal transformation $\bar{O}_{i j}$

$$
\boldsymbol{r}_{i}=\sum_{v} \bar{O}_{i v} \bar{R}_{v}, \quad \boldsymbol{k}_{i}=\sum_{v} \bar{O}_{i v} \boldsymbol{K}_{v}
$$

where the first $A-2$ coordinates $\overline{\boldsymbol{R}}_{j}$ and their Bargmann space transforms $\overline{\boldsymbol{K}}_{j}$ refer to the $f-1$ and $A-f-1$ internal coordinates of fragments $f$ and $A-f$, while the remaining two are the relative coordinate vector $\boldsymbol{R}$, eq. (2), and the c.m. vector $\boldsymbol{R}_{\mathrm{c} . \mathrm{m} .}$. For examples, see fig. 1. Similarly, for the cluster decomposition into fragments $f^{\prime}$ and $A-f^{\prime}$, coordinates $\boldsymbol{R}_{j}$ can be introduced by a different orthonormal transformation $O_{i j}$

$$
\boldsymbol{r}_{i}=\sum_{\sigma} O_{i \sigma} \boldsymbol{R}_{\sigma}, \quad \boldsymbol{k}_{i}^{*}=\sum_{\sigma} O_{i \sigma} \boldsymbol{K}_{\sigma}^{*}
$$

Using the orthogonality of $\bar{O}$ (and similarly of $O$ )

$$
\prod_{i=1}^{A} A\left(\overline{\boldsymbol{K}}_{i}, \boldsymbol{r}_{i}\right)=\pi^{-3 A / 4} \exp \left\{\sum_{i=1}^{A}\left(-\frac{1}{2} \overline{\boldsymbol{K}}_{i} \cdot \overline{\boldsymbol{K}}_{i}-\frac{1}{2} \overline{\boldsymbol{R}}_{i} \cdot \overline{\boldsymbol{K}}_{i}+\sqrt{2} \overline{\boldsymbol{K}}_{i} \cdot \overline{\boldsymbol{R}}_{i}\right)\right\},
$$

and the BS transform of the operator $\mathcal{O} Y$ can be written

$$
H\left(\bar{K}, K^{*}\right)=\int \mathrm{d} r_{1} \ldots \int \mathrm{d} \boldsymbol{r}_{A} \prod_{i=1}^{A} A\left(\overline{\boldsymbol{K}}_{i}, \overline{\boldsymbol{K}}_{i}\right) \cup Y \prod_{j=1}^{A} A\left(\boldsymbol{K}_{j}^{*}, \boldsymbol{R}_{j}\right)
$$

where both the $\overline{\boldsymbol{R}}_{i}$ and $\boldsymbol{R}_{j}$ would have to be considered as functions of $\boldsymbol{r}_{1}, \ldots, \boldsymbol{r}_{A}$ if one were to attempt a direct evaluation of the $3 A$-dimensional integral.

An SU(3) coupled Bargmann space function can be defined by

$$
\left[P\left(\overline{\boldsymbol{K}}_{1}\right)^{\left(Q_{1} 0\right)} \times P\left(\overline{\boldsymbol{K}}_{2}\right)^{\left(Q_{2} 0\right)}\right]_{\alpha}^{(\lambda \mu)}=\sum_{\alpha_{1} \alpha_{2}}\left\langle\left(Q_{1} 0\right) \alpha_{1}\left(Q_{2} 0\right) \alpha_{2} \mid(\lambda \mu) \alpha\right\rangle P\left(\overline{\boldsymbol{K}}_{1}\right)_{\alpha_{1}}^{\left(Q_{1} 0\right)} P\left(\overline{\boldsymbol{K}}_{2}\right)_{\alpha_{2}}^{\left(Q_{2} 0\right)},
$$

where the subgroup labels in the full $S U(3)$ coupling coefficient can be chosen in any convenient basis. A product of two $A$-functions can then be expanded ${ }^{24,25}$ ) by

$$
\begin{aligned}
A\left(\overline{\boldsymbol{K}}_{1}, \overline{\boldsymbol{R}}_{1}\right) A\left(\overline{\boldsymbol{K}}_{2}, \overline{\boldsymbol{R}}_{2}\right)=\sum_{Q_{1} Q_{2}} \sum_{(\lambda \mu) \alpha}[ & P\left(\overline{\boldsymbol{K}}_{1}\right)^{\left(Q_{1} 0\right)} \times \\
& \left.P\left(\overline{\boldsymbol{K}}_{2}\right)^{\left(\boldsymbol{Q}_{2} 0\right)}\right]_{\alpha}^{(\lambda \mu)} \\
& \times\left[\chi\left(\overline{\boldsymbol{R}}_{1}\right)^{\left(Q_{1} 0\right)} \times \chi\left(\overline{\boldsymbol{R}}_{2}\right)^{\left(\boldsymbol{Q}_{2} 0\right)}\right]_{\alpha}^{(\lambda \mu) *} .
\end{aligned}
$$

This is the key to the evaluation of the matrix elements of $\mathcal{O} Y$ in an SU(3) coupled cluster basis, when generalized to the appropriate number of products of $A$-functions.

Since oscillator excitations associated with most of the internal degrees of freedom of the fragments are restricted to 0s states, most of the $A\left(\overline{\boldsymbol{K}}_{i}, \overline{\boldsymbol{R}}_{i}\right)$ and $A\left(\boldsymbol{K}_{j}^{*}, \boldsymbol{R}_{j}\right)$ expansions can be frozen in their zeroth-order (unit) terms. For a cluster system with 2 heavy fragments the oscillator excitations of a few additional internal degrees of freedom are fixed in their lowest Pauli-allowed (shell-model) values, while oscillator excitations associated with the relative motion degree of freedom, $\boldsymbol{R}$, can be arbitrarily 
high. For the ${ }^{12} \mathrm{C}+{ }^{12} \mathrm{C}$ cluster system, e.g., (see fig. 1b)

$$
\begin{aligned}
& \prod_{i=1}^{A} A\left(\overline{\boldsymbol{K}}_{i},\right.\left.\overline{\boldsymbol{R}}_{i}\right)=\sum_{\left(\lambda_{c} \mu_{c}\right)} \sum_{Q} \sum_{(\lambda \mu)} \sum_{\alpha} \\
& \times\left[\left[\left[P\left(\overline{\boldsymbol{K}}_{1}\right)^{(40)} \times P\left(\overline{\boldsymbol{K}}_{2}\right)^{(40)}\right]^{(04)} \times\left[P\left(\overline{\boldsymbol{K}}_{3}\right)^{(40)} \times P\left(\overline{\boldsymbol{K}}_{4}\right)^{(40)}\right]^{(04)}\right]^{\left(\lambda_{c} \mu_{c}\right)} \times P(\overline{\boldsymbol{K}})^{(\mathbf{Q})}\right]_{\alpha}^{(\lambda \mu)} \\
& \times\left[\left[\left[\chi\left(\overline{\boldsymbol{R}}_{1}\right)^{(40)} \times \chi\left(\overline{\boldsymbol{R}}_{2}\right)^{(40)}\right]^{(04)} \times\left[\chi\left(\overline{\boldsymbol{R}}_{3}\right)^{(40)} \times \chi\left(\overline{\boldsymbol{R}}_{4}\right)^{(40)}\right]^{(04)}\right]^{\left(\lambda_{c} \mu_{c}\right)} \times \chi(\overline{\boldsymbol{R}})^{(\mathbf{Q 0})}\right]_{\alpha}^{(\lambda \mu) *}
\end{aligned}
$$

+ terms leading to Pauli-forbidden excitations + terms leading to internal excitations of the ${ }^{12} \mathrm{C}$ fragments

$$
\begin{aligned}
\equiv \sum_{\left(\lambda_{c} \mu_{c}\right)} \sum_{Q} \sum_{\left(\lambda \mu_{)}\right.} \sum_{\alpha}[[ & P\left(\overline{\boldsymbol{K}}_{1}, \ldots\right)^{\left(\lambda_{f} \mu_{f}\right)} \times P\left(\boldsymbol{K}_{3}, \ldots\right)^{\left.\left(\lambda_{A}-f^{\left.\mu_{A}-f\right)}\right]^{\left(\lambda_{c} \mu_{c}\right)} \times P(\boldsymbol{K})^{(Q 0)}\right]_{\alpha}^{(\lambda \mu)}} \\
\times & {\left[\left[\chi\left(\overline{\boldsymbol{R}}_{1}, \ldots\right)^{\left(\lambda_{f} \mu_{f}\right)} \times \chi\left(\overline{\boldsymbol{R}}_{3}, \ldots\right)^{\left(\lambda_{A}-f \mu_{A}-f\right)}\right]^{\left(\lambda_{c} \mu_{c}\right)} \times \chi(\overline{\boldsymbol{R}})^{(Q 0)}\right]_{\alpha}^{(\lambda \mu) *}+\ldots }
\end{aligned}
$$

where the last shorthand notation can be used for any binary fragment decomposition involving internal degrees of freedom $\overline{\boldsymbol{R}}_{1}, \overline{\boldsymbol{R}}_{2}, \ldots ; \overline{\boldsymbol{R}}_{i}, \overline{\boldsymbol{R}}_{i+1}, \ldots ;$ with Bargmann space transforms $\overline{\boldsymbol{K}}_{1}, \overline{\boldsymbol{K}}_{2} ; \ldots ; \overline{\boldsymbol{K}}_{i}, \overline{\boldsymbol{K}}_{i+1}, \ldots ;$ and a single degree of freedom $\overline{\boldsymbol{R}}$ (without index) with Bargmann space transform $\bar{K}$, describing the relative motion of the two fragments. [In the ${ }^{12} \mathrm{C}+{ }^{12} \mathrm{C}$ system the internal $K$-space functions can in fact be expressed in terms of functions of the two pseudovectors $\left[\boldsymbol{K}_{1} \times \boldsymbol{K}_{2}\right]$ and $\left[\boldsymbol{K}_{3} \times \boldsymbol{K}_{4}\right]$, see eq. (B.5). $]$. In general, the $\mathrm{BS}$ transform of the operator $\mathcal{O} Y$ can be expanded by

$$
\begin{aligned}
& H\left(\bar{K}, K^{*}\right)=\sum_{\left(\lambda_{c} \mu_{c}\right) Q(\lambda \mu) \alpha} \sum_{\left(\lambda_{c}^{\prime} \mu_{c}^{\prime}\right) Q^{\prime}\left(\mu^{\prime \prime} \mu^{\prime}\right) \alpha^{\prime \prime}}\left[\left[P\left(\bar{K}_{1}, \ldots\right)^{\left(\lambda_{f} \mu_{f}\right)} \times P\left(\widetilde{K}_{i}, \ldots\right)^{\left(\lambda_{A}-f^{\mu_{A}-f}\right)}\right]^{\left(\lambda_{c} \mu_{c}\right)}\right. \\
& \left.\times P(\bar{K})^{(\boldsymbol{Q} 0)}\right]_{\alpha}^{(\lambda \mu)}\left[\left[P\left(K_{1}^{*}, \ldots\right)^{\left(\mu_{f^{\prime}} \lambda^{\prime}\right)} \times P\left(K_{j}^{*}, \ldots\right)^{\left(\mu_{A}-f^{\prime} \lambda_{A}-f^{\prime}\right)}\right]^{\left(\mu_{c}^{\prime} \lambda_{c}^{\prime}\right)} \times P\left(K^{*}\right)^{\left(0 Q^{\prime}\right)}\right]_{\alpha^{\prime \prime}}^{\left(\mu^{\prime \prime} \lambda^{\prime \prime}\right)} \\
& \times\left\langle[ [ \chi ( \overline { \boldsymbol { R } } _ { 1 } , \ldots ) ^ { ( \lambda _ { f } \mu _ { f } ) } \times \chi ( \overline { \boldsymbol { R } } _ { i } , \ldots ) ^ { ( \lambda _ { A } - f _ { A } - f ) } ] ^ { ( \lambda _ { c } \mu _ { c } ) } \times \chi ( \overline { R } ) ^ { ( \boldsymbol { Q } 0 ) } ] _ { \alpha } ^ { ( \lambda \mu ) } | \mathcal { O } Y | \left[\left[\chi\left(\boldsymbol{R}_{1}, \ldots\right)^{\left(\lambda_{f}^{\prime} \mu_{f^{\prime}}\right)}\right.\right.\right. \\
& \left.\left.\left.\times \chi\left(\boldsymbol{R}_{j}, \ldots\right)^{\left(\lambda_{A}-f^{\prime} \mu_{A}-f^{\prime}\right)}\right]^{\left(\lambda_{c}^{\prime} \mu_{c}^{\prime}\right)} \times \chi(\boldsymbol{R})^{\left(Q^{\prime} 0\right)}\right]_{\alpha^{\prime \prime}}^{\left(\lambda^{\prime \prime} \mu^{\prime \prime}\right)}\right\rangle \text {. }
\end{aligned}
$$

If the operator $\mathcal{O}$ is the hamiltonian, it is useful to imagine that $\mathcal{O}$ has been expanded in terms of SU(3) irreducible tensor operators and to write eq. (27) in terms of the SU(3) reduced matrix elements of such operators. Examples of this technique have been given in ref. ${ }^{24}$ ). If $\mathcal{O}$ is the unit operator, (or any SU(3) scalar operator), the matrix elements of eq. (27) must be diagonal in $(\lambda \mu)$ and $\alpha$ and be independent of $\alpha$. Making use of this property and the relation

$$
\left[P(\bar{K})^{(\lambda \mu)} \times P\left(K^{*}\right)^{(\mu \lambda)}\right]_{000}^{(00)}=\frac{1}{[d(\lambda \mu)]^{\ddagger}} \sum_{\alpha} P(\bar{K})_{\alpha}^{(\lambda \mu)} P\left(K^{*}\right)_{\alpha}^{(\mu \lambda)}
$$

with $d(\lambda \mu)=$ the dimension of the representation $(\lambda \mu)$, see eq. (A.6); the BS transform 
of the unit operator can be written

$$
\begin{aligned}
& H\left(\overline{\boldsymbol{K}}, \boldsymbol{K}^{*}\right)=\sum_{\left(\lambda_{c} \mu_{c}\right) Q} \sum_{\left(\lambda_{c}^{\prime} \mu_{c}^{\prime}\right) Q^{\prime}} \sum_{(\lambda \mu)}[d(\lambda \mu)]^{\frac{1}{2}} \\
& \times\left[\left[\left[P\left(\bar{K}_{1}, \ldots\right)^{\left(\lambda_{f} \mu_{f}\right)} \times P\left(\bar{K}_{i}, \ldots\right)^{\left(\lambda_{A}-f \mu_{A}-s\right)}\right]^{\left(\lambda_{c} \mu_{c}\right)} \times P(\bar{K})^{(Q 0)}\right]^{\left(\lambda_{\mu}\right)}\right.
\end{aligned}
$$

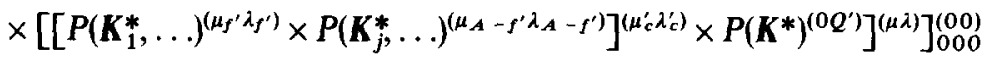

$$
\begin{aligned}
& \times\left\langle\left[\left[\chi\left(\overline{\boldsymbol{R}}_{1}, \ldots\right)^{\left(\lambda_{f} \mu_{f}\right)} \times \chi\left(\overline{\boldsymbol{R}}_{\boldsymbol{i}}, \ldots\right)^{\left(\lambda_{A}-f \mu_{A}-f\right)}\right]^{\left(\lambda_{c} \mu_{c}\right)} \times \chi(\overline{\boldsymbol{R}})^{(Q 0)}\right]_{\alpha}^{(\lambda \mu)}\right| \\
& \times Y\left|\left[\left[\chi\left(\boldsymbol{R}_{1}, \ldots\right)^{\left(\lambda_{f^{\prime}} \boldsymbol{\mu}^{\prime}\right)} \times \chi\left(\boldsymbol{R}_{j}, \ldots\right)^{\left(\lambda_{A}-f^{\prime} \mu_{A}-f^{\prime}\right)}\right]^{\left(\lambda_{c}^{\prime} \mu_{c}^{\prime}\right)} \times \chi(\boldsymbol{R})^{\left(Q^{\prime} 0\right)}\right]_{\alpha}^{(\lambda \mu)}\right\rangle .
\end{aligned}
$$

The coefficients of the SU(3) coupled $K$-space functions with specific $\left(\lambda_{f} \mu_{f}\right)$, $\left(\lambda_{A-s} \mu_{A-f}\right),\left(\lambda_{c} \mu_{c}\right),(Q 0),\left(\lambda_{f}, \mu_{f^{\prime}}\right),\left(\lambda_{A-f^{\prime}} \mu_{A-f^{\prime}}\right)\left(\lambda_{c}^{\prime} \mu_{c}^{\prime}\right),\left(Q^{\prime} 0\right)$; and $(\lambda \mu)$ are the orbital parts of the desired norm or overlap matrix elements.

The calculation of norm or overlap matrix elements can thus be accomplished by two basic steps;

(i) the BS transform of the unit operator is expressed in terms of the appropriate Bargmann space variables $\overline{\boldsymbol{K}}_{i}, \overline{\boldsymbol{K}}, \boldsymbol{K}_{j}^{*}$, and $\boldsymbol{K}^{*}$; and

(ii) these functions are then expanded in terms of the appropriately $\mathrm{SU}(3)$ coupled $K$-space functions. The coefficient of the

$$
\begin{aligned}
{[d(\lambda \mu)]^{\frac{1}{2}}\left[\left[\left[P\left(\overline{\boldsymbol{K}}_{1}, \ldots\right) \times \ldots\right]^{\left(\lambda_{c} \mu_{c}\right)} \times P(\overline{\boldsymbol{K}})^{(\boldsymbol{Q} 0)}\right]^{(\lambda \mu)} \times[[\right.} & \left.P\left(\boldsymbol{K}_{1}^{*}, \ldots\right) \times, . .\right]^{\left(\mu_{c}^{\prime} \lambda^{\prime}\right)} \\
& \left.\left.\times P\left(\boldsymbol{K}^{*}\right)^{\left(\Omega Q^{\prime}\right)}\right]^{(\mu \lambda)}\right]_{000}^{(00)}
\end{aligned}
$$

term gives the numerical value of the desired norm or overlap matrix element. The usefulness of this technique has recently also been exploited by Fujiwara and Horiuchi $\left.{ }^{27}\right)$. In its use of the generating function property of $A(K, R)$, it is ak in in philosophy to that used earlier by Suzuki ${ }^{51}$ ) and Horiuchi ${ }^{34}$ ).

[It should perhaps also be noted that both the operator $Y$ and the separate fragment internal functions $P\left(K_{1}, . .\right)^{\left(\lambda_{r} \mu_{F}\right)}, P\left(K_{i}, \ldots\right)^{\left(\lambda_{A}-\kappa_{A}-r\right)}$ must be properly normalized. In the detailed application of the technique these normalizations are included during the process of calculation. Thus, norms tend to unity for separated fragments, i.e. in the limit $Q \rightarrow \infty$.]

\subsection{CALCULATION OF $H\left(\boldsymbol{K}, K^{*}\right)$}

In single-particle $k$-space coordinates the BS transform of the operator $1 \cdot Y$ has the simple form of eq. (18). In principle, the transformation to the appropriate Bargmann space variables $\overline{\boldsymbol{K}}_{i}, \ldots, \boldsymbol{K}_{j}^{*}$ is straightforward. In practice, it is complicated because of the large number of terms in the expansion of the Young operator, even though this can be drastically reduced by using a double coset decomposition ${ }^{52}$ ) for $Y$. The transformation from $\overline{\boldsymbol{k}}_{i} \rightarrow \overline{\boldsymbol{K}}_{i}, \boldsymbol{k}_{j}^{*} \rightarrow \boldsymbol{K}_{j}^{*}$ does not change the simple gaussian 
form of $H$, and

$$
H\left(\overrightarrow{\boldsymbol{K}}, \boldsymbol{K}^{*}\right)=\sum_{\beta} a_{\beta} \exp \left\{\sum_{i, j} \sigma_{i j}(\beta)\left(\overline{\boldsymbol{K}}_{i} \cdot \boldsymbol{K}_{j}^{*}\right)\right\},
$$

where, in place of a sum over $A$ ! permutation operators, the $\beta$-sum has been restricted to a sum over double coset generators with weighting coefficients $a_{\beta}$. For a cluster system of two heavy fragments, each consisting of several subunits with 0 s internal excitation, the number of terms in the $\beta$-sum is still much too large for practical purposes, so that a more indirect method will be used for the evaluation of $H\left(\bar{K}, K^{*}\right)$.

The general form of eq. (31) is its most important feature. The SU(3) scalar character of $H\left(\overline{\mathbf{K}}, \boldsymbol{K}^{*}\right)$ follows from

$$
\left(\bar{K}_{i} \cdot K_{j}^{*}\right)=\sqrt{3}\left[P\left(\bar{K}_{i}\right)^{(10)} \times P\left(K_{j}^{*}\right)^{(01)}\right]_{000}^{(00)}
$$

and its iteration

$$
\left(\bar{K}_{i} \cdot K_{j}^{*}\right)^{n}=n ![d(n, 0)]^{\frac{1}{1}}\left[P\left(\bar{K}_{i}\right)^{(n 0)} \times P\left(K_{j}^{*}\right)^{(0 n)}\right]_{000}^{(00)}
$$

Of the many internal degrees of freedom most are restricted to 0s states, and expansions of the exponentials of the corresponding $\bar{K}_{i}, \boldsymbol{K}_{j}^{*}$ are frozen in their zerothorder unit terms. Only a few internal degrees of freedom carry oscillator excitations, and these carry excitations fixed in their lowest Pauli-allowed values, limiting the expansion of the exponentials in these $\boldsymbol{K}_{i}$ variables to specific (small) values. Only the oscillator excitations associated with the relative motion Bargmann space variables $\bar{K}, K^{*}$ (without index) are completely unrestricted. It is thus useful to factor $H\left(\bar{K}, K^{*}\right)$ into three types of terms:

(i) Terms carrying internal excitations only:

$$
\exp \left\{\sum_{i, j} \sigma_{i j}\left(\overline{\boldsymbol{K}}_{i} \cdot \boldsymbol{K}_{j}^{*}\right)\right\}
$$

When expanded, these exponentials lead only to terms of the form

$$
\left[P\left(\bar{K}_{1}, \bar{K}_{2}, \ldots\right)^{\left(\lambda^{\prime} \mu^{\prime}\right)} \times P\left(K_{1}^{*}, K_{2}^{*}, \ldots\right)^{\left(\mu^{\prime} \lambda^{\prime}\right)}\right]_{000}^{(00)}
$$

where we have used the SU(3) (00) character of (34). The representations $\left(\lambda^{\prime} \mu^{\prime}\right)$ are limited to a few possibilities since the number of oscillator quanta associated with $\left(\lambda^{\prime} \mu^{\prime}\right)$ must be smaller than those associated with both $\left(\lambda_{c} \mu_{c}\right)$ and $\left(\lambda_{c}^{\prime} \mu_{c}^{\prime}\right)$.

(ii) Cross terms involving both internal and relative motion degrees of freedom:

$$
\exp \left\{\sum_{i}\left[\sigma_{i 0}\left(\overline{\boldsymbol{K}}_{i} \cdot \boldsymbol{K}^{*}\right)+\sigma_{0 i}\left(\overline{\boldsymbol{K}} \cdot \boldsymbol{K}_{i}^{*}\right)\right]\right\} \text {. }
$$

When expanded, these exponentials lead to terms of the form

$$
\left[P\left(\bar{K}_{1}, \bar{K}_{2}, \ldots\right)^{(m 0)} \times P\left(K^{*}\right)^{(0 m)}\right]_{000}^{(00)} \cdot\left[P(\bar{K})^{\left(m^{\prime} 0\right)} \times P\left(K_{1}^{*}, K_{2}^{*}, \ldots\right)^{\left(0 m^{\prime}\right)}\right]_{000}^{(00)} .
$$

The possible integers $m$ and $m^{\prime}$ are restricted by the requirements

$$
\begin{aligned}
\left(\lambda^{\prime} \mu^{\prime}\right) \times(m 0) & \rightarrow\left(\lambda_{c} \mu_{c}\right), \\
\left(\lambda^{\prime} \mu^{\prime}\right) \times\left(m^{\prime} 0\right) & \rightarrow\left(\lambda_{c}^{\prime} \mu_{c}^{\prime}\right),
\end{aligned}
$$


i.e., the excitations associated with the fragment internal degrees of freedom must couple to resultant SU(3) symmetries $\left(\lambda_{c} \mu_{c}\right)$ and $\left(\lambda_{c}^{\prime} \mu_{c}^{\prime}\right)$. With fragment $f=f^{\prime}$, (e.g. in any norm matrix element), $\left(\lambda_{c} \mu_{c}\right)$ and $\left(\lambda_{c}^{\prime} \mu_{c}^{\prime}\right)$ must carry the same number of internal oscillator excitations, and hence $m=m^{\prime}$ in this case. For overlap matrix elements between different cluster decompositions; i.e. for $f \neq f^{\prime}$ the difference $m-m^{\prime}$ is a fixed integer. For a ${ }^{12} \mathrm{C}+{ }^{12} \mathrm{C}-{ }^{20} \mathrm{Ne}+\alpha$ overlap matrix element, e.g., the ${ }^{12} \mathrm{C}+{ }^{12} \mathrm{C}$ SU(3) representation $\left(\lambda_{c} \mu_{c}\right)$ carries $8+8=16$ internal oscillator quanta, while the ${ }^{20} \mathrm{Ne}+\alpha$ representation carries $20+0$ internal oscillator quanta, so that $m^{\prime}-m=4$ in this case. In general

$$
m^{\prime}=m+\Delta .
$$

(iii) The final factor contains only the relative motion $K$-space variable and has the form

$$
\exp \left\{\sigma_{00}(p)\left(\overline{\boldsymbol{K}} \cdot \boldsymbol{K}^{*}\right)\right\},
$$

where the coefficient $\sigma_{00}(p)$ is a function of the fragment masses and the index $p$ which counts the number of particles exchanged between fragments. The term in $H\left(\overline{\boldsymbol{k}}_{i}, \boldsymbol{k}_{i}^{*}\right)$ corresponding to the exchange of nucleons $a$ and $b$ can be written, [in single-particle $k$-space coordinates; with $P=(a b)]$, as

$$
\exp \left\{\sum_{i=1}^{A}\left(\overline{\boldsymbol{k}}_{i} \cdot P \boldsymbol{k}_{i}^{*}\right)\right\}=\exp \left\{\sum_{i=1}^{A}\left(\overline{\boldsymbol{k}}_{i} \cdot \boldsymbol{k}_{i}^{*}\right)-\left(\overline{\boldsymbol{k}}_{a}-\overline{\boldsymbol{k}}_{b}\right) \cdot\left(\boldsymbol{k}_{a}^{*}-\boldsymbol{k}_{b}^{*}\right)\right\} .
$$

For fragments of mass numbers $f$ and $A-f$, the single-particle $\overline{\boldsymbol{k}}_{i}$ transform into: for $i=1, \ldots, f$

$$
\overline{\boldsymbol{k}}_{i}=\frac{1}{[f]^{\frac{1}{2}}}\left(-\left[\frac{A-f}{A}\right]^{\frac{1}{2}} \overline{\boldsymbol{K}}+\left[\frac{f}{A}\right]^{-\frac{1}{2}} \boldsymbol{K}_{\mathbf{c} \cdot \mathrm{m} .}\right)+\ldots
$$

for $j=f+1, \ldots, A$

$$
\overline{\boldsymbol{k}}_{j}=\frac{1}{[A-f]^{\frac{1}{2}}}\left(\left[\frac{f}{A}\right]^{\frac{1}{2}} \overline{\boldsymbol{K}}+\left[\frac{A-f}{A}\right]^{\frac{1}{2}} \boldsymbol{K}_{\text {c.m. }}\right)+\ldots
$$

while the single-particle $\boldsymbol{k}_{i}^{*}$, corresponding to a cluster decomposition into fragments $f^{\prime}$ and $A-f^{\prime}$, transform into:

for $i=1, \ldots, f^{\prime}$

$$
\boldsymbol{k}_{i}^{*}=\frac{1}{\left[f^{\prime}\right]^{\frac{1}{2}}}\left(-\left[\frac{A-f^{\prime}}{A}\right]^{\frac{1}{2}} \boldsymbol{K}^{*}+\left[\frac{f^{\prime}}{A}\right]^{\frac{1}{2}} \boldsymbol{K}_{\mathrm{c} . \mathrm{m} .}^{*}\right)+\ldots,
$$

for $j=f^{\prime}+1, \ldots, A$

$$
\boldsymbol{k}_{j}^{*}=\frac{1}{\left[A-f^{\prime}\right]^{\frac{1}{2}}}\left(\left[\frac{f^{\prime}}{A}\right]^{\frac{1}{2}} \boldsymbol{K}^{*}+\left[\frac{A-f^{\prime}}{A}\right]^{\frac{1}{2}} \boldsymbol{K}_{\mathrm{c} . \mathrm{m} .}^{*}\right)+\ldots
$$

where the terms abbreviated by $+\ldots$ stand for the $A-2 \bar{K}_{i}$ and $\boldsymbol{K}_{i}^{*}$ associated with the fragment internal degrees of freedom which can make no contribution to $\sigma_{00}$. 
Without loss of generality, assume that $a<b, f^{\prime}<f$. Then

$$
\begin{gathered}
\left(\bar{k}_{a}-\bar{k}_{b}\right) \cdot\left(k_{a}^{*}-k_{b}^{*}\right)=0+\ldots \quad \text { unless } a<f^{\prime}, b>f, \\
\left(\bar{k}_{a}-\bar{k}_{b}\right) \cdot\left(\boldsymbol{k}_{a}^{*}-\boldsymbol{k}_{b}^{*}\right)=\frac{A}{\left[f(A-f) f^{\prime}\left(A-f^{\prime}\right)\right]^{\frac{3}{2}}}\left(\overline{\boldsymbol{K}} \cdot \boldsymbol{K}^{*}\right)+\ldots \text { if } a<f^{\prime}, b>f,
\end{gathered}
$$

while

$$
\sum_{i=1}^{A}\left(\overline{\boldsymbol{k}}_{i} \cdot \boldsymbol{k}_{i}^{*}\right)=\left(\boldsymbol{K}_{\mathrm{c} . \mathrm{m} .} \cdot \boldsymbol{K}_{\mathrm{c} . \mathrm{m}}^{*}\right)+\left[\frac{f^{\prime}(A-f)}{f\left(A-f^{\prime}\right)}\right]^{\frac{1}{*}}\left(\overline{\boldsymbol{K}} \cdot \boldsymbol{K}^{*}\right)+\ldots
$$

Terms denoted by $+\ldots$ are associated with internal degrees of freedom and have already been accounted for in factors (i) and (ii).

For a permutation involving $p$ pairs of nucleons with indices $a<f^{\prime}, b>f$,

$$
\sigma_{00}(p)=\frac{f^{\prime}(A-f)-p A}{\left[f(A-f) f^{\prime}\left(A-f^{\prime}\right)\right]^{\frac{1}{2}}} .
$$

The full $H\left(\vec{K}, K^{*}\right)$ can be assembled from combinations of products involving factors of types (i), (ii) and (iii), weighted with as yet undetermined coefficients which can be functions of $m, m^{\prime},\left(\lambda^{\prime} \mu^{\prime}\right)$, and $p$. Since $m-m^{\prime}$ is a fixed integer, $m^{\prime}$ can be eliminated as a redundant variable. The $K$-space functions $P^{\left(\lambda^{\prime} \mu^{\prime}\right)}$ are built from fragment internal degrees of freedom only. Since these are independent of the number of exchanged nucleons $(p)$, the $\left(\lambda^{\prime} \mu^{\prime}\right)$ dependence of the coefficients can be factored from the $p$-dependence, and the full function $H\left(\bar{K}, K^{*}\right)$ can be assembled by means of $m\left(\lambda^{\prime} \mu^{\prime}\right)$ dependent coefficients $c_{m}\left(\lambda^{\prime} \mu^{\prime}\right)$ and $m, p$-dependent coefficients $D_{m}(p)$ to yield

$$
\begin{aligned}
H\left(\overline{\boldsymbol{K}}, \boldsymbol{K}^{*}\right)= & \sum_{m\left(\lambda^{\prime} \mu^{\prime}\right)} \frac{c_{m\left(\lambda^{\prime} \mu^{\prime}\right)}}{\left[d(m 0) d\left(m^{\prime} 0\right) d\left(\lambda^{\prime} \mu^{\prime}\right)\right]^{\frac{1}{2}}} \\
& \times\left[\sum_{p} D_{m}(p) \exp \left\{\frac{f^{\prime}(A-f)-p A}{\left[f(A-f) f^{\prime}\left(A-f^{\prime}\right)\right]^{\frac{1}{2}}}\left(\overline{\boldsymbol{K}} \cdot \boldsymbol{K}^{*}\right)\right\}\right] \\
& \times \sum_{\alpha, \beta, \gamma} P\left(\overline{\boldsymbol{K}}_{1}, \overline{\boldsymbol{K}}_{2}, \ldots\right)_{\alpha}^{(m 0)} P\left(\boldsymbol{K}^{*}\right)_{\alpha}^{(0 m)} P(\overline{\boldsymbol{K}})_{\beta}^{\left(m^{\prime} 0\right)} P\left(\boldsymbol{K}_{1}^{*}, \boldsymbol{K}_{2}^{*}, \ldots\right)_{\beta}^{\left(0 m^{\prime}\right)} \\
& \times P\left(\overline{\boldsymbol{K}}_{1}, \overline{\boldsymbol{K}}_{2}, \ldots\right)_{\gamma}^{\left(\lambda^{\prime} \mu^{\prime}\right)} P\left(\boldsymbol{K}_{1}^{*}, \boldsymbol{K}_{2}^{*}, \ldots\right)_{\gamma}^{\left(\mu^{\prime} \lambda^{\prime}\right)},
\end{aligned}
$$

where factors of type (i) and (ii) have been expanded by means of eq. (28) in terms of the set of subgroup labels $\alpha, \beta$ and $\gamma$. The coupling of the internal functions to resultant $\mathrm{SU}(3)$ symmetries $\left(\lambda_{c} \mu_{c}\right)$ and $\left(\lambda_{c}^{\prime} \mu_{c}^{\prime}\right)$ leads to

$$
\begin{aligned}
H\left(\overline{\boldsymbol{K}}, \boldsymbol{K}^{*}\right)= & \left.\sum_{m\left(\lambda^{\prime} \mu^{\prime}\right)} \frac{c_{m\left(\lambda^{\prime} \mu^{\prime}\right)}}{\left[d(m 0) d\left(m^{\prime} 0\right) d\left(\lambda^{\prime} \mu^{\prime}\right)\right]^{\frac{1}{t}}}\left[\sum_{p} D_{m}(p) \exp \left\{\frac{f^{\prime}(A-f)-p A}{\left[f(A-f) f^{\prime}\left(A-f^{\prime}\right)\right]^{\frac{1}{z}}} \overline{\boldsymbol{K}} \cdot \boldsymbol{K}^{*}\right)\right\}\right] \\
& \times \sum_{\alpha, \beta, \gamma} \sum_{\left(\lambda_{c} \mu_{c}\right) \alpha_{c}} \sum_{\left(\lambda_{c}^{\prime} \mu_{c}^{\prime}\right) \alpha_{c}^{\prime}}\left\langle(m 0) \alpha ;\left(\lambda^{\prime} \mu^{\prime}\right) \gamma \mid\left(\lambda_{c} \mu_{c}\right) \alpha_{c}\right\rangle\left\langle\left(m^{\prime} 0\right) \beta ;\left(\lambda^{\prime} \mu^{\prime}\right) \gamma \mid\left(\lambda_{c}^{\prime} \mu_{c}^{\prime}\right) \alpha_{c}^{\prime}\right\rangle \\
& \times n\left(m\left(\lambda^{\prime} \mu^{\prime}\right)\left(\lambda_{c} \mu_{c}\right)\right) n\left(m^{\prime}\left(\lambda^{\prime} \mu^{\prime}\right)\left(\lambda_{c}^{\prime} \mu_{c}^{\prime}\right)\right) P\left(\bar{K}_{1}, \bar{K}_{2}, \ldots\right)_{\alpha_{c}}^{\left(\lambda_{c} \mu_{c}\right)} \\
& \times P\left(\boldsymbol{K}_{1}^{*}, \boldsymbol{K}_{2}^{*}, \ldots\right)_{\alpha_{c}^{\prime}}^{\left(\mu^{\prime} \lambda_{c}^{\prime}\right)} P\left(\boldsymbol{K}^{*}\right)_{\alpha}^{(0 m)} P(\bar{K})_{\beta}^{\left(m^{\prime} 0\right)}
\end{aligned}
$$


For the purpose of evaluating the coefficients the subgroup labels in the full SU(3) Wigner coefficients are best chosen in terms of the intrinsic basis $\alpha \equiv \varepsilon_{\alpha} \Lambda_{\alpha} M_{A_{\alpha}}, \ldots$. The constants $n\left(m\left(\lambda^{\prime} \mu^{\prime}\right)\left(\lambda_{c} \mu_{c}\right)\right)$ are renormalization coefficients associated with the combination of $P\left(K_{\text {internal }}\right)$ and are built from the $\left[\left(Q_{1}+Q_{2}\right) ! / Q_{1} ! Q_{2} !\right]^{\frac{1}{2}}$ renormalization coefficients associated with the coupling of Bargmann space $K$-functions in the same variable, [see eq. (B.1)]. The details of this renormalization are unimportant since the renormalization factors can be absorbed by the as yet unknown coefficients $c_{m\left(\lambda^{\prime} \mu^{\prime}\right)}$. It is most convenient to redefine these in terms of the new coefficients $y_{m\left(\lambda^{\prime} \mu^{\prime}\right)}$; where (with $m^{\prime}=m+\Delta$ )

$$
\begin{aligned}
\frac{c_{m\left(\lambda^{\prime} \mu^{\prime} \mu^{\prime}\right.}\left(m\left(\lambda^{\prime} \mu^{\prime}\right)\left(\lambda_{c} \mu_{c}\right)\right) n\left(m^{\prime}\left(\lambda^{\prime} \mu^{\prime}\right)\left(\lambda_{c}^{\prime} \mu_{c}^{\prime}\right)\right)}{\left[d(m 0) d\left(m^{\prime} 0\right) d\left(\lambda^{\prime} \mu^{\prime}\right)\right]^{\frac{1}{2}}} \equiv y_{m\left(\lambda^{\prime} \mu^{\prime}\right)}[m !(m+\Delta) !]^{\frac{1}{2}} & {\left[\frac{A}{f(A-f)}\right]^{(m+\Delta) / 2} } \\
& \times\left[\frac{A}{f^{\prime}\left(A-f^{\prime}\right)}\right]^{m / 2}
\end{aligned}
$$

With this definition the structure constants $y_{m\left(\lambda^{\prime} \mu^{\prime}\right)}$, have the simplest normalization.

It will now be useful to express the BS transform $H\left(\bar{K}, K^{*}\right)$ in three basic forms:

In form I the internal and relative motion $K$-space functions arc retained in their uncoupled form with subgroup labels $\alpha, \beta, \alpha_{c}, \alpha_{c}^{\prime}$ specified in a cartesian oscillator or intrinsic $\varepsilon \Lambda M_{A}$ basis. This form is the one most closely related to a single-particle basis, and it is this form which is used for the evaluation of the coefficients $y_{m\left(\lambda^{\prime} \mu^{\prime}\right)}$ and $D_{m}(p)$.

In form II, $H\left(\bar{K}, K^{*}\right)$ is then given in terms of the basic SU(3) coupled $K$-space functions of eq. (30) in which all dependence on subgroup labels has disappeared. Once the $y_{m\left(\lambda^{\prime} \mu^{\prime}\right)}$ and $D_{m}(p)$ are known, this form gives norm and overlap matrix elements directly since these are merely the coefficients in the expansions in terms of the SU(3) coupled functions (30). Form II is therefore one of the key results of this investigation.

In form III, the BS transform is also expanded in terms of SU(3) invariant coupled functions. The order of the SU(3) coupling is now slightly different. This form is most useful for the evaluation of the single-column BS transforms, since the SU(3) invariants of form III can be expanded most easily in terms of scalar products of the type $\left(\bar{K}_{i} \cdot K_{j}^{*}\right)$.

The combination of eqs. (46), (47) and (48) leads directly to

Form I

$$
\begin{aligned}
H\left(\overline{\boldsymbol{K}}, \boldsymbol{K}^{*}\right) & =\sum_{m\left(\lambda^{\prime} \mu^{\prime}\right)} y_{m\left(\lambda^{\prime} \mu^{\prime}\right)}[m !(m+\Delta) !]^{\frac{1}{2}}\left[\frac{A}{f(A-f)}\right]^{(m+\Delta) / 2}\left[\frac{A}{f^{\prime}\left(A-f^{\prime}\right)}\right]^{m / 2} \\
& \times\left[\sum_{D} D_{m}(p) \exp \left\{\frac{f^{\prime}(A-f)-p A}{\left[f(A-f) f^{\prime}\left(A-f^{\prime}\right)\right]^{\frac{1}{2}}}\left(\overline{\boldsymbol{K}} \cdot \boldsymbol{K}^{*}\right)\right\}\right] \sum_{\alpha, \beta, \gamma} P\left(\boldsymbol{K}^{*}\right)_{\tilde{\alpha}}^{(0 m)} P(\overline{\boldsymbol{K}})_{\beta}^{(m+\Delta, 0)} \\
& \times \sum_{\left(\lambda_{c} \mu_{c}\right) \alpha_{c}} \sum_{\left(\lambda_{c}^{\prime} \mu_{c}^{\prime}\right) \alpha_{c}^{\prime}}\left\langle(m 0) \alpha ;\left(\lambda^{\prime} \mu^{\prime}\right) \gamma \mid\left(\lambda_{c} \mu_{c}\right) \alpha_{c}\right\rangle\left\langle(m+\Delta, 0) \beta ;\left(\lambda^{\prime} \mu^{\prime}\right) \gamma \mid\left(\lambda_{c}^{\prime} \mu_{c}^{\prime}\right) \alpha_{c}^{\prime}\right\rangle \\
& \times P\left(\overline{\boldsymbol{K}}_{1}, \overline{\boldsymbol{K}}_{2}, \ldots\right)_{\alpha_{c}}^{\left(\lambda_{c} \mu_{c}\right)} P\left(\boldsymbol{K}_{1}^{*}, \boldsymbol{K}_{2}^{*}, \ldots\right)_{\tilde{\alpha} c^{\prime}}^{\left(\mu_{c}^{\prime} \lambda_{c}^{\prime}\right)}
\end{aligned}
$$


where this form of $H\left(\bar{K}, K^{*}\right)$ will be most useful for the evaluation of the coefficients $y_{m\left(\lambda^{\prime} \mu^{\prime}\right)}$ and $D_{m}(p)$. For binary fragment systems the internal function of symmetry $\left(\lambda_{c} \mu_{c}\right)$ is to be built by coupling fragment functions of specific symmetries $\left(\lambda_{f} \mu_{f}\right)$ and $\left(\lambda_{A-f} \mu_{A-f}\right)$ as in eqs. (26) and (27). Similarly for functions of symmetry $\left(\lambda_{c}^{\prime} \mu_{\mathrm{c}}^{\prime}\right)$.

Alternately, form II is achieved as follows. Starting with eq. (46), the exponentials are first expanded in powers of $\left(\overline{\boldsymbol{K}} \cdot \boldsymbol{K}^{*}\right)^{Q-m}$. Four $9-(\lambda \mu)$ transformations involving recouplings

$$
\begin{aligned}
& {\left[[(m 0) \times(0 m)]^{(00)} \times[(Q-m, 0) \times(0, Q-m)]^{(00)}\right]^{(00)}} \\
& \rightarrow\left[[(m 0) \times(Q-m, 0)]^{(00)} \times(0 Q)\right]^{(00)}, \\
& {\left[\left[\left(m^{\prime} 0\right) \times\left(0 m^{\prime}\right)\right]^{(00)} \times\left[\left(\lambda^{\prime} \mu^{\prime}\right) \times\left(\mu^{\prime} \lambda^{\prime}\right)\right]^{(00)}\right]^{(00)} \rightarrow\left[\left[\left(m^{\prime} 0\right) \times\left(\lambda^{\prime} \mu^{\prime}\right)\right]^{\left(\lambda_{c}^{\prime} \mu_{c}^{\prime}\right)} \times\left(\mu_{c}^{\prime} \lambda_{c}^{\prime}\right)\right]^{(00)},} \\
& {\left[[(Q 0) \times(0 Q)]^{(00)} \times\left[\left(\lambda_{c}^{\prime} \mu_{c}^{\prime}\right) \times\left(\mu_{c}^{\prime} \lambda_{c}^{\prime}\right)\right]^{(00)}\right]^{(00)}} \\
& \rightarrow\left[\left[(Q 0) \times\left(\lambda_{c}^{\prime} \mu_{c}^{\prime}\right)\right]^{(\lambda \mu)} \times\left[(0 Q) \times\left(\mu_{c}^{\prime} \lambda_{c}^{\prime}\right)\right]^{(\mu \lambda)}\right]^{(00)}, \\
& {\left[[(Q-m, 0) \times(m 0)]^{(Q 0)} \times\left[(m+\Delta, 0) \times\left(\lambda^{\prime} \mu^{\prime}\right)\right]^{\left(\lambda_{c}^{\prime} \mu_{c}^{\prime}\right)}\right]^{(\lambda \mu)}} \\
& \rightarrow\left[[(Q-m, 0) \times(m+\Delta, 0)]^{(Q+\Delta, 0)} \times\left[(m 0) \times\left(\lambda^{\prime} \mu^{\prime}\right)\right]^{\left(\lambda_{c} \mu_{c}\right)}\right]^{(\lambda \mu)},
\end{aligned}
$$

then lead to the expansion of $H\left(\bar{K}, K^{*}\right)$ which is in the basic form of eq. (29), needed for the direct evaluation of the norm or overlap matrix elements. Some of the technical details of SU(3) recoupling transformations are discussed in appendix A. The first three transformations, each with three (00) representations, are given in terms of trivial SU(3) dimension factors, [eq. (A.16)]. The 4th transformation is a bona fide 9-( $\lambda \mu)$ transformation made up of multiplicity-free SU(3) couplings only. A number of renormalizations of $K$-space functions are needed, see eqs. (B.1) and (47); and phase factors are introduced by a change in the order of some $\mathrm{SU}(3)$ couplings, see eq. (A.3b). With these factors the BS transform can be put into the basic form:

\section{Form II}

$$
\begin{aligned}
& H\left(\bar{K}, K^{*}\right)=\sum_{m\left(\lambda^{\prime} \mu^{\prime}\right)} y_{m\left(\lambda^{\prime} \mu^{\prime}\right)} \sum_{Q=m}^{\infty}\left[\frac{A}{f(A-f)}\right]^{(m+\Delta) / 2}\left[\frac{A}{f^{\prime}\left(A-f^{\prime}\right)}\right]^{m / 2} \\
& \times \sum_{\left(\lambda_{c} \mu_{c}\right)\left(\lambda_{c}^{\prime} \mu_{c}^{\prime}\right)}\left\{\sum_{p=0}^{f^{\prime}} D_{m}(p)\left[\frac{f^{\prime}(A-f)-p A}{\left[f(A-f) f^{\prime}\left(A-f^{\prime}\right)\right]^{\frac{1}{2}}}\right]^{Q-m}\right\} \frac{[Q !(Q+\Delta) !]^{\frac{1}{2}}}{(Q-m) !} \\
& \times \sum_{(\lambda \mu)}(-1)^{\Delta+\lambda_{c}+\mu_{c}+\lambda_{c}^{\prime}+\mu_{c}^{\prime}}\left[\begin{array}{lll}
(Q-m, 0) & (m 0) & (Q 0) \\
(m+\Delta, 0) & \left(\lambda^{\prime} \mu^{\prime}\right) & \left(\lambda_{c}^{\prime} \mu_{c}^{\prime}\right) \\
(Q+\Delta, 0) & \left(\lambda_{c} \mu_{c}\right) & (\lambda \mu)
\end{array}\right][d(\lambda \mu)]^{\frac{1}{2}} \\
& \times\left[\left[P\left(\bar{K}_{1}, \bar{K}_{2}, \ldots\right)^{\left(\lambda_{c} \mu_{c}\right)} \times P(\bar{K})^{(Q+\Delta, 0)}\right]^{(\lambda \mu)} \times\left[P\left(K_{1}^{*}, K_{2}^{*}, \ldots\right)^{\left(\mu_{c}^{\prime} \lambda_{c}^{\prime}\right)} \times P\left(K^{*}\right)^{(0 Q)}\right]^{(\mu \lambda)}\right]_{000}^{(00)}
\end{aligned}
$$


Comparison with eq. (29) gives the matrix element

$$
\begin{aligned}
\langle[ & {\left.\left[\left(\lambda_{f} \mu_{f}\right) \times\left(\lambda_{A-f} \mu_{A-f}\right)\right]\left(\lambda_{c} \mu_{c}\right) \times(Q+\Delta, 0)\right](\lambda \mu) \alpha|Y|\left[\left[\left(\lambda_{f^{\prime}} \mu_{f^{\prime}}\right)\right.\right.} \\
& \left.\left.\left.\times\left(\lambda_{A-f^{\prime}} \mu_{A-f^{\prime}}\right)\right]\left(\lambda_{c}^{\prime} \mu_{c}^{\prime}\right) \times(Q 0)\right](\lambda \mu) \alpha\right\rangle \\
= & \sum_{m\left(\lambda^{\prime} \mu^{\prime}\right)} y_{m\left(\lambda^{\prime} \mu^{\prime}\right)}\left[\frac{A}{f(A-f}\right]^{(m+\Delta) / 2}\left[\frac{A}{f^{\prime}\left(A-f^{\prime}\right)}\right]^{m / 2} \frac{[Q !(Q+\Delta) !]^{\frac{1}{2}}}{(Q-m) !}(-1)^{\Delta+\lambda_{c}+\mu_{c}+\lambda_{c}^{\prime}+\mu_{c}^{\prime}} \\
& \times\left\{\sum_{p=0}^{f^{\prime}} D_{m}(p)\left[\frac{f^{\prime}(A-f)-p A}{\left[f(A-f) f^{\prime}\left(A-f^{\prime}\right)\right]^{\frac{1}{3}}}\right]^{Q-m}\right\}\left[\begin{array}{lll}
(Q-m, 0) & (m 0) & (Q 0) \\
(m+\Delta, 0) & \left(\lambda^{\prime} \mu^{\prime}\right) & \left(\lambda_{c}^{\prime} \mu_{c}^{\prime}\right) \\
(Q+\Delta, 0) & \left(\lambda_{c} \mu_{c}\right) & (\lambda \mu)
\end{array}\right]
\end{aligned}
$$

An alternate form, involving an expansion in terms of SU(3) Racah or 6-( $(\lambda \mu)$ coefficients, (see appendix A), gives

$$
\begin{aligned}
& \left\langle\left[\left[\left(\lambda_{f} \mu_{f}\right) \times\left(\lambda_{A-f} \mu_{A-f}\right)\right]\left(\lambda_{c} \mu_{c}\right) \times(Q+\Delta, 0)\right](\lambda \mu) \alpha\right| Y \\
& \quad \times\left|\left[\left[\left(\lambda_{f^{\prime}} \mu_{f^{\prime}}\right) \times\left(\lambda_{A-f^{\prime}} \mu_{A-f^{\prime}}\right)\right]\left(\lambda_{c}^{\prime} \mu_{c}^{\prime}\right) \times(Q 0)\right](\lambda \mu) \alpha\right\rangle \\
& =(-1)^{Q+\lambda+\mu^{+}+\lambda_{c}^{\prime}+\mu_{c}^{\prime}}\left[\frac{d(Q 0) d\left(\lambda_{c} \mu_{c}\right)}{d(\lambda \mu)}\right]_{m\left(\lambda^{\prime} \mu^{\prime}\right)}^{\frac{1}{2}} y_{m\left(\lambda^{\prime} \mu^{\prime}\right)}\left[\frac{A}{f(A-f)}\right]^{(m+\Delta) / 2}\left[\frac{A}{f^{\prime}\left(A-f^{\prime}\right)}\right]^{m / 2} . \\
& \quad \times \frac{[Q !(Q+\Delta) !]^{\frac{1}{2}}}{d(m 0)(Q-m) !}\left\{\sum_{p=0}^{f^{\prime}} D_{m}(p)\left[\frac{f^{\prime}(A-f)-p A}{\left[f(A-f) f^{\prime}\left(A-f^{\prime}\right)\right]^{\frac{1}{2}}}\right]^{Q-m}\right\} \\
& \quad \times \sum_{\left(\lambda^{\prime \prime} \mu^{\prime \prime}\right) \rho}\left[d\left(\lambda^{\prime \prime} \mu^{\prime \prime}\right)\right]^{\frac{1}{2}} U\left(\left(\lambda_{c} \mu_{c}\right)\left(\mu^{\prime} \lambda^{\prime}\right)\left(\lambda^{\prime \prime} \mu^{\prime \prime}\right)(0 m+\Delta) ;(m 0)_{--} ;\left(\mu_{c}^{\prime} \lambda_{c}^{\prime}\right)_{-} \rho\right) \\
& \quad \times U\left((Q 0)(0 Q-m)\left(\lambda^{\prime \prime} \mu^{\prime \prime}\right)(0 m+\Delta) ;(m 0)_{-} ;(0 Q+\Delta) \quad\right) \\
& \quad \times U\left((Q+\Delta, 0)\left(\lambda_{c} \mu_{c}\right)(Q 0)\left(\mu_{c}^{\prime} \lambda_{c}^{\prime}\right) ;(\lambda \mu)_{--} ;\left(\lambda^{\prime \prime} \mu^{\prime \prime}\right) \rho_{-}\right) .
\end{aligned}
$$

For the special case $f^{\prime}=f$, (equal fragment decomposition in bra and ket), this is in the form of eq. (27) of ref. ${ }^{25}$ ), with $\left(\lambda_{c} \mu_{c}\right)=\left(\lambda_{c}^{\prime} \mu_{c}^{\prime}\right), \Delta=0$. Once the structure and exchange coefficients $y_{m\left(\lambda^{\prime} \mu^{\prime}\right)}$ and $D_{m}(p)$ have been evaluated, norm and overlap matrix elements follow at once from this simple, universal formula. In ref. ${ }^{25}$ ) the $y_{m\left(\lambda^{\prime} \mu^{\prime}\right)}$ are tabulated for a few simple examples, $(\alpha+$ heavy fragment cluster norms). The method used there for the evaluation of the $y_{m\left(\lambda^{\prime} \mu^{\prime}\right)}$ is rather indirect, making use of shell-model techniques. A more direct method will be developed in this work.

Before proceeding, it will be useful to exhibit one more form of the BS transform $H\left(\bar{K}, \bar{K}^{*}\right)$. With a somewhat different recoupling path, eq. (46) leads to:

Form III

$$
\begin{aligned}
H\left(\overline{\boldsymbol{K}}, \boldsymbol{K}^{*}\right)= & \sum_{m\left(\lambda^{\prime} \mu^{\prime}\right)} y_{m\left(\lambda^{\prime} \mu^{\prime}\right)}[m !(m+\Delta) !]^{\frac{1}{2}}\left[\frac{A}{f(A-f)}\right]^{(m+\Delta) / 2}\left[\frac{A}{f^{\prime}\left(A-f^{\prime}\right)}\right]^{m / 2} \\
& \times\left[\sum_{p=0}^{f^{\prime}} D_{m}(p) \exp \left\{\frac{f^{\prime}(A-f)-p A}{\left[f(A-f) f^{\prime}\left(A-f^{\prime}\right)\right]^{\frac{1}{2}}}\left(\overline{\boldsymbol{K}} \cdot \boldsymbol{K}^{*}\right)\right\}\right]
\end{aligned}
$$




$$
\begin{aligned}
& \times \sum_{\left(\lambda_{c} \mu_{c}\right)\left(\lambda_{c} \mu_{c}^{\prime}\right)}(-1)^{\lambda_{c}+\mu_{c}+\lambda_{c}^{\prime}+\mu_{c}^{\prime}} \sum_{\left(\lambda^{\prime \prime} \mu^{\prime \prime}\right) \rho}(-1)^{\lambda^{\prime \prime}+\mu^{\prime \prime}}\left[\frac{d\left(\lambda^{\prime \prime} \mu^{\prime \prime}\right) d\left(\lambda_{c} \mu_{c}\right)}{d(m 0)}\right]^{\frac{1}{2}} \\
& \times U\left(\left(\lambda_{c} \mu_{c}\right)\left(\mu^{\prime} \lambda^{\prime}\right)\left(\lambda^{\prime \prime} \mu^{\prime \prime}\right)(0 m+\Delta) ;(m 0)_{-} ;\left(\mu_{c}^{\prime} \lambda_{c}^{\prime}\right)_{-} \rho\right) \\
& \times\left[\left[P\left(\bar{K}_{1}, \bar{K}_{2}, \ldots\right)^{\left(\lambda_{c} \mu_{c}\right)} \times P\left(K_{1}^{*}, K_{2}^{*}, \ldots\right)^{\left(\mu_{c}^{\prime} \lambda_{c}^{\prime}\right)}\right]^{\left(\lambda^{\prime \prime} \mu^{\prime \prime}\right) \rho}\right. \\
& \left.\times\left[P\left(\bar{K}^{(m+\Delta, 0}\right) \times P\left(K^{*}\right)^{(0 m)}\right]^{\left(\mu^{\prime \prime} \lambda^{\prime \prime}\right)}\right]_{000}^{(00)}
\end{aligned}
$$

This is the generalization of eqs. (15) and (23) of ref. ${ }^{25}$ ).

In lighter nuclei the $y_{m\left(\lambda^{\prime} \mu^{\prime}\right)}$ can be calculated directly for the full $A$-particle system of arbitrary space symmetry, characterized by full 4-columned Young tableaux. An example will be given in sect. 4 . In heavier nuclei the total number of $y_{m\left(\lambda^{\prime} \mu^{\prime}\right)}$ needed for the evaluation of eq. (51) can become somewhat large. In heavier nuclei, therefore, a variant of the above technique is used. BS transforms are first calculated for $l+n$ particle subsystems of space symmetries characterized by single-columned Young tableaux, (totally antisymmetric space symmetries). The BS transforms for the full $A$-particle system are then built by suitable symmetrized combinations of these single-column functions. For this reason, as well as for simplicity, the calculation of the $y_{m\left(\lambda^{\prime} \mu^{\prime}\right)}$ will be illustrated first by the example of such a "single-column function". For an $l+n$ fragment system with totally antisymmetric spatial wave function, (made up of neutrons with spin-up only, e.g.), the $Y$ operator is a simple $l+n$-particle antisymmetrizer, $\mathscr{A}$. The cluster function in the bra and ket sides of the matrix element will be assumed to consist of fragments of $l+n$ and $l^{\prime}+n^{\prime}$ particles, respectively. Form I of the BS transform, eq. (49), with $K$-space functions expressed in a cartesian oscillator or intrinsic $\varepsilon \Lambda M_{A}$ basis is most useful for the evaluation of the $y_{m\left(\lambda^{\prime} \mu^{\prime}\right)}$. With specific choices for the subgroup labels, $\alpha_{c}, \alpha_{c}^{\prime}$, the oscillator excitations carried by the internal degrees of freedom can be uniquely specified since the fragment SU(3) symmetries are uniquely determined by the particle number for totally antisymmetric states carrying minimum Pauli-allowed excitations. For $n=1,2,3,4 ; 5$ and 6 , the fragment $\left(\lambda_{n} \mu_{n}\right)$ must be (00), (10), (01), (00); (20) and (21). The $n=3$ fragment, e.g. must consist of one 0 s particle and two p-shell particles, coupled antisymmetrically to SU(3) symmetry (01). An $l+n=2+3$-particle cluster system of this type, with $\left(\lambda_{1} \mu_{l}\right)=(10),\left(\lambda_{n} \mu_{n}\right)=(01)$, can have $\left(\lambda_{c} \mu_{c}\right)=(00)$ or (11) only. With $\left(\lambda_{c} \mu_{c}\right)=(11)$ and $\alpha_{c} \equiv \varepsilon_{c} \Lambda_{c} M_{A_{c}}=3 \frac{1}{2} \frac{1}{2}$, e.g., this must carry two $z$-excitations and one $x$-excitation. These can be extracted from a BS transform by means of eq. (19) by differentiation with respect to a $k_{i_{z}}$, where $i$ is a single-particle index associated with the $l=2$ fragment, and further differentiations with respect to $k_{j_{z}}$ and $k_{m_{x}}$, where $j$ and $m$ are single-particle indices associated with the $n=3$ fragment.

Using the orthonormality of the $P\left(\bar{K}_{1}, \bar{K}_{2}, \ldots\right)_{\alpha_{c}}^{\left(\hat{\lambda}_{c} \mu_{c}\right)}$ in the complex Bargmann space with integration measure, $\mathrm{d} g\left(k_{i \beta}\right)$, given by eq. (12),

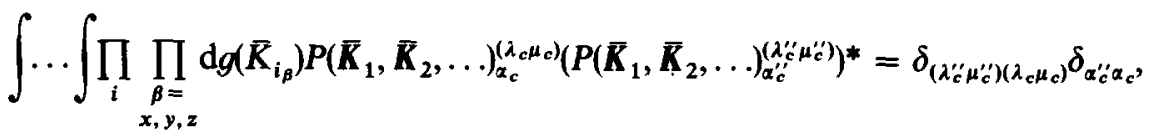


eq. (49) can be converted to a function of the relative motion $\overline{\boldsymbol{K}}$ and $\boldsymbol{K}^{*}$ only

$$
\begin{aligned}
& \int \ldots \int \prod_{i \alpha} \prod_{j \beta} \mathrm{d} g\left(\bar{K}_{i_{\alpha}}\right) \mathrm{d} g\left(K_{j_{\beta}}\right)\left(P\left(\overline{\boldsymbol{K}}_{1}, \overline{\boldsymbol{K}}_{2}, \ldots\right)_{\alpha_{c}}^{\left(\lambda_{c} \mu_{c}\right)}\right)^{*} H\left(P\left(\boldsymbol{K}_{1}^{*}, \boldsymbol{K}_{2}^{*}, \ldots . \mu_{\alpha_{c}^{\prime}}^{\left(\mu_{c}^{\prime} \lambda_{c}^{\prime} c\right)}\right)^{*}\right. \\
& =\mathscr{H}\left(\overline{\boldsymbol{K}}, \boldsymbol{K}^{*} \boldsymbol{\gamma}_{\alpha_{c}, \alpha_{c}^{\prime}}^{\left.\lambda_{c} \mu_{c}\right),\left(\lambda_{c}^{\prime} \mu_{c}^{\prime}\right)}\right. \text {. }
\end{aligned}
$$

Except for a trivial overall multiplicative constant, this $\mathscr{H}\left(\overline{\boldsymbol{K}}, \boldsymbol{K}^{*}\right)$ is identical with a function of $\overline{\boldsymbol{K}}$ and $\boldsymbol{K}^{*}$ obtained by a superposition of the operations

$$
\left[\prod_{i \alpha j \beta}\left[\frac{1}{n_{i_{\alpha}} ! n_{j_{\beta}} !}\right]^{\frac{1}{2}} \frac{\partial^{n_{i_{\alpha}}}}{\partial \bar{k}_{i_{\alpha}}^{n_{i_{\alpha}}}} \frac{\partial^{n_{j_{\beta}}}}{\partial k_{j_{\beta}}^{* n_{j_{\beta}}}}\left\{\sum_{\boldsymbol{P}}(-1)^{\sigma(P)} \exp \left[\sum_{s=1}^{l+n}\left(\overline{\boldsymbol{k}}_{s} \cdot P \boldsymbol{k}_{s}^{*}\right)\right]\right\}\right]_{\overline{\mathbf{K}}_{i}=0, \mathbf{K}_{j}^{*}=0, \mathbf{K}_{\mathrm{c} . \mathrm{m} .}=0}
$$

The above operations involve the following steps:

(a) Differentiation with respect to single-particle $k$-space coordinates of the BS transform of the $(l+n)$-particle antisymmetrizers, eq. (18), with $c_{P}=(-1)^{\sigma(P)}$; $\sigma(P)=$ parity of $P$.

(b) Transformation of the resultant functions to functions of $\bar{K}, \overline{\boldsymbol{K}}_{1}, \overline{\boldsymbol{K}}_{2}, \ldots, \boldsymbol{K}^{*}$, $K_{i}^{*}, \ldots, K_{j}^{*}$ by means of eq. (20) [see also eqs. (41)], for the $\overline{\boldsymbol{k}}_{i}$; and by means of eq. (21) [see also eqs. (42)], for the $\boldsymbol{k}_{j}^{*}$. In the $\bar{k}_{i}$, particle indices $i=1, \ldots, l$ belong to fragment $f, i=l+1, \ldots, l+n$ to fragment $A-f$. In the $k_{j}^{*}$, particle indices $j=1, \ldots, l^{\prime}$ belong to fragment $f^{\prime}, j=l^{\prime}+1 \ldots, l^{\prime}+n^{\prime}$ to fragment $A-f^{\prime}$.

(c) In the last step, $\boldsymbol{K}_{\text {c.m. }}$ and all $\overline{\boldsymbol{K}}_{i}$ and $\boldsymbol{K}_{j}^{*}$ describing fragment internal degrees of freedom are set equal to zero. [ $K_{\text {c.m. }}$ is the $K$-space variable for the c.m. degree of freedom of the whole nucleus.]

The required superposition of such operations involves the coupling of the $l+n$ particle internal functions to fragment and channel $\mathrm{SU}(3)$ symmetries $\left(\lambda_{t} \mu_{t}\right),\left(\lambda_{n} \mu_{n}\right)$, $\left(\lambda_{c} \mu_{c}\right),\left(\lambda_{l^{\prime}} \mu_{l^{\prime}}\right),\left(\lambda_{n^{\prime}} \mu_{n^{\prime}}\right)$, and $\left(\lambda_{c}^{\prime} \mu_{c}^{\prime}\right)$. For single-column (totally antisymmetric) functions, only very simple $S U(3)$ Wigner coefficients are needed, and in many cases a clever choice of $\alpha_{c}$ and $\alpha_{c}^{\prime}$ can restrict the superposition to a single term (e.g. by selection of a unique SU(3) coupling path for which all Wigner coefficients are equal to +1 ). In connection with the operations defined by eq. (56) it will be useful to define a symbol of $l+n$ and $l^{\prime}+n^{\prime}$ single-particle indices. E.g., with $l=3 ; n=3 ; l^{\prime}=1, n^{\prime}=5$ :

$$
\begin{aligned}
& F\left(\begin{array}{c}
0 ; \bar{x} \bar{x} ; 0 \bar{z} \bar{x} \\
0 ; 0 z^{*} x^{*} z^{* 2}
\end{array}\right) \equiv\left[\frac{\partial}{\partial \bar{k}_{2 z}} \frac{\partial}{\partial \bar{k}_{3 x}} \frac{\partial}{\partial \bar{k}_{5_{z}}} \frac{\partial}{\partial \bar{k}_{6_{x}}} \frac{\partial}{\partial k_{3_{z}}^{*}} \frac{\partial}{\partial k_{4_{x}}^{*}}\right. \\
& \left.\times \frac{\partial}{\partial k_{5_{y}}^{*}} \frac{1}{\sqrt{2} !} \frac{\partial^{2}}{\partial k_{6_{z}}^{* 2}}\left\{\sum_{\boldsymbol{P}}(-1)^{\sigma(P)} \exp \left[\sum_{i=1}^{6}\left(\bar{k}_{i} \cdot P \boldsymbol{k}_{i}^{*}\right)\right]\right\}\right]_{\overline{\boldsymbol{k}}_{i}=0, \mathbf{K}_{j}^{*}=0, \mathbf{K}_{\text {c.m. }}=0}
\end{aligned}
$$

A computer code has been written to calculate the functions of $\boldsymbol{K}$ and $\boldsymbol{K}^{*}$ resulting from these operations ${ }^{53}$ ). A fairly extensive tabulation of such functions is given in appendix $\mathrm{D}$. 


\subsection{A SPECIFIC EXAMPLE}

To illustrate the method in detail the following specific example is chosen: a 4particle system characterized by a single-columned Young tableau with cluster decomposition into fragments with $l+n=l^{\prime}+n^{\prime}=1+3$ particles. This 4-particle system is characterized by $\mathrm{SU}(3)$ symmetries

$$
\left[\left(\lambda_{l} \mu_{l}\right) \times\left(\lambda_{n} \mu_{n}\right)\right]\left(\lambda_{c} \mu_{c}\right)=\left[\left(\lambda_{l}, \mu_{l^{\prime}}\right) \times\left(\lambda_{n^{\prime}} \mu_{n^{\prime}}\right)\right]\left(\lambda_{c}^{\prime} \mu_{c}^{\prime}\right)=[(00) \times(01)](01) .
$$

For this simple system there are only two possible values of the indices $m\left(\lambda^{\prime} \mu^{\prime}\right)$ :

(i) $m=0, \quad\left(\lambda^{\prime} \mu^{\prime}\right)=(01)\left(=\left(\lambda_{c} \mu_{c}\right)=\left(\lambda_{c}^{\prime} \mu_{c}^{\prime}\right)\right)$,

(ii) $m=1, \quad\left(\lambda^{\prime} \mu^{\prime}\right)=(10)$.

Except for an overall multiplicative constant which can be absorbed by the coefficients $y_{m\left(\lambda^{\prime} \mu^{\prime}\right)}$ the function $\mathscr{H}\left(\bar{K}, \boldsymbol{K}^{*}\right)_{\alpha_{c}, \alpha_{c}^{\prime}}^{\left(\lambda_{c} \mu_{c}\right),\left(\lambda_{c}^{\prime} \mu_{c}^{\prime}\right)}$ defined by eq. (55), with $\alpha_{c}=\alpha_{c}^{\prime}=$ $\varepsilon_{c} A_{c} M_{A_{c}}=1 \frac{1}{2} \frac{1}{2}\left[\mathrm{SU}(3)\right.$ highest weight for $\left.\left(\lambda_{c} \mu_{c}\right)=(01)\right]$ is identical with the single $F$-function

That is

$$
F\left(\begin{array}{l}
0 ; 0 \bar{z} \bar{x} \\
\left.0 ; 0 z^{*} x^{*}\right)
\end{array}\right)
$$

$$
\begin{aligned}
& \sum_{m\left(\lambda^{\prime} \mu^{\prime}\right)} y_{m\left(\lambda^{\prime} \mu^{\prime}\right)} m !\left[\frac{A}{f(A-f)}\right]^{m}\left[\sum_{P} D_{m}(p) \exp \left\{-p \frac{A}{f(A-f)}\left(\bar{K} \cdot K^{*}\right)\right\}\right] \\
& \times \sum_{\alpha, \beta, \gamma}\left\langle(m 0) \alpha ;\left(\lambda^{\prime} \mu^{\prime}\right) \gamma \mid(01) 1 \frac{1}{2} \frac{1}{2}\right\rangle\left\langle(m 0) \beta ;\left(\lambda^{\prime} \mu^{\prime}\right) \gamma(01) 1 \frac{1}{2} \frac{1}{2}\right\rangle P(\bar{K})_{\beta}^{(m 0)} P\left(K^{*}\right)_{\alpha}^{(0 m)} \\
& =y_{0(01)}\left[\sum_{p} D_{0}(p) \exp \left\{-p \frac{A}{f(A-f)}\left(\overline{\boldsymbol{K}} \cdot \boldsymbol{K}^{*}\right)\right\}\right] P(\overline{\boldsymbol{K}})_{000}^{(00)} P\left(\boldsymbol{K}^{*}\right)_{000}^{(00)} \\
& +y_{1(10)}\left[\sum_{p} D_{1}(p) \exp \left\{-p \frac{A}{f(A-f)}\left(\bar{K} \cdot K^{*}\right)\right\}\right] \\
& \times\left\{\left\langle(10) 200 ;(10)-1 \frac{1}{2} \frac{1}{2}\right\}(01) 1 \frac{1}{2} \frac{1}{2}\right\rangle^{2} P(\bar{K})_{200}^{(10)}\left(P(K)_{200}^{(10)}\right)^{*} \\
& \left.+\left\langle(10)-1 \frac{1}{2} \frac{1}{2} ;(10) 200 \mid(01) 1 \frac{1}{2} \frac{1}{2}\right\rangle^{2} P(\bar{K})_{-1 \frac{1}{2}}^{(10)}\left(P(K)_{-1 \frac{1}{2}}^{(10)}\right)^{*}\right\} \\
& =\left\{1-\exp \left(-\frac{A}{f(A-f)}\left(\overline{\boldsymbol{K}} \cdot \boldsymbol{K}^{*}\right)\right)\left[1+\frac{A}{f(A-f)}\left(\bar{K}_{z} K_{z}^{*}+\bar{K}_{x} K_{x}^{*}\right)\right]\right\},
\end{aligned}
$$

where the value of the $F$-function has been read from table 9, (appendix $D$ ). The needed $P_{\beta}^{(m 0)}$ are: $P(\bar{K})_{000}^{(00)}=1, P(\bar{K})_{200}^{(10)}=\bar{K}_{z}, P(\bar{K})_{-1}^{(10)}=\bar{K}_{x}$. The two non-trivial SU(3) Wigner coefficients have absolute value $\sqrt{\frac{1}{2}}$. With these, eq. (58) leads at once to the determination of the $y_{m\left(\lambda^{\prime} \mu^{\prime}\right)}$ and $D_{m}(p)$.
(i) $y_{0(01)}=1$,
$D_{0}(0)=1$,
$D_{0}(1)=-1$,
(ii) $y_{1(10)}=2$,
$D_{1}(0)=0$,
$D_{1}(1)=-1$. 
The evaluation of the coefficients $y_{m\left(\lambda^{\prime} \mu^{\prime}\right)}$ and $D_{m}(p)$ through the functions $\mathscr{H}(\bar{K}$, $\left.K^{*}\right)_{\alpha_{c}, \alpha_{c}^{\prime}}^{\left(\lambda_{c} \mu_{c}\right),\left(\lambda_{c}^{\prime} \mu_{c}^{\prime}\right)}$ thus makes use of the specific construction of fragment internal functions in an intrinsic or cartesian oscillator basis. Once these coefficients have been evaluated, however, it is vital to make use of one of the SU(3) invariant (SU(3) coupled) forms of $H\left(\bar{K}, K^{*}\right)$. For present purposes form III is to be preferred. In the application of form III to the present simple example the only non-trivial $U$-coefficient, (with $m=1,\left(\lambda^{\prime} \mu^{\prime}\right)=(10),\left(\lambda^{\prime \prime} \mu^{\prime \prime}\right)=(11)$ ), has the value $-\frac{1}{2}$; (using eqs. (A.8) and (A.11), e.g.). Eq. (53) leads to

$$
\begin{aligned}
H\left(\overline{\mathbf{K}}, \boldsymbol{K}^{*}\right)^{(01),(01)}=\mathrm{e}^{\eta \overline{\mathbf{K}} \cdot \mathbf{K}^{*}}\left\{( 1 - \operatorname { e x p } [ - \frac { A } { f ( A - f ) } ( \overline { \boldsymbol { K } } \cdot \boldsymbol { K } ^ { * } ) ] ) \sqrt { 3 } \left[P\left(\overline{\boldsymbol{K}}_{1}, \overline{\boldsymbol{K}}_{2}\right)^{(01)}\right.\right. \\
\left.\times P\left(\boldsymbol{K}_{1}^{*}, \boldsymbol{K}_{2}^{*}\right)^{(10)}\right]_{000}^{(00)} \\
\quad-2 \frac{A}{f(A-f)} \exp \left[-\frac{A}{f(A-f)}\left(\overline{\boldsymbol{K}} \cdot \boldsymbol{K}^{*}\right)\right]\left(\left[\left[P\left(\overline{\boldsymbol{K}}_{1}, \overline{\boldsymbol{K}}_{2}\right)^{(01)}\right.\right.\right. \\
\left.\left.\quad \times P\left(\boldsymbol{K}_{1}^{*}, \boldsymbol{K}_{2}^{*}\right)^{(10)}\right]^{(00)} \times\left[P(\overline{\boldsymbol{K}})^{(10)} \times P\left(\boldsymbol{K}^{*}\right)^{(01)}\right]^{(00)}\right]_{000}^{(00)} \\
\left.-\frac{1}{2} \sqrt{8}\left[\left[P\left(\overline{\boldsymbol{K}}_{1}, \overline{\boldsymbol{K}}_{2}\right)^{(01)} \times P\left(\boldsymbol{K}_{1}^{*}, \boldsymbol{K}_{2}^{*}\right)^{(10)}\right]^{(11)} \times\left[P(\overline{\boldsymbol{K}})^{(10)} \times P\left(\boldsymbol{K}^{*}\right)^{(01)}\right]^{(11)}\right]_{000}^{(00)}\right\}, \quad(59)
\end{aligned}
$$

where the internal $\overline{\boldsymbol{K}}_{1}, \overline{\boldsymbol{K}}_{2}$ associated with the heavier fragment are illustrated in fig. 1a, and where

$$
P\left(\bar{K}_{1}, \bar{K}_{2}\right)^{(01)}=\left[P\left(\bar{K}_{1}\right)^{(10)} \times P\left(\bar{K}_{2}\right)^{(10)}\right]^{(01)}=\bar{K}_{12}=\sqrt{\frac{1}{2}}\left[\bar{K}_{1} \times \bar{K}_{2}\right] .
$$

SU(3) coupled functions of the type appearing in eq. (59) can be expanded in terms of simple scalar products. A list of such expansions is given as part of appendix B. E.g., eq. (B.10) gives

$$
\begin{aligned}
\sqrt{8}\left[\left[P\left(\bar{K}_{1}, \bar{K}_{2}\right)^{(01)} \times P\left(K_{1}^{*}, K_{2}^{*}\right)^{(10)}\right]^{(11)} \times\left[P(\bar{K})^{(10)} \times P\left(K^{*}\right)^{(01)}\right]^{(11)}\right]_{000}^{(00)} \\
=\left(\bar{K} \cdot \bar{K}_{12}\right)\left(K^{*} \cdot K_{12}^{*}\right)-\frac{1}{3}\left(\bar{K} \cdot K^{*}\right)\left(\bar{K}_{12} \cdot K_{12}^{*}\right),
\end{aligned}
$$

where it is important to note that $\bar{K}_{12}$ is a pseudovector, (SU(3)(01) tensor), in applying eq. (B.10) to this case. Eq. (61) together with eq. (32) transforms eq. (59) to the form

$$
\begin{aligned}
& H^{(01)(01)}=\exp \eta\left(\overline{\boldsymbol{K}} \cdot \boldsymbol{K}^{*}\right)\left\{\left(\overline{\boldsymbol{K}}_{12} \cdot \boldsymbol{K}_{12}^{*}\right)\left(1-\exp \left[-\frac{A}{f(A-f)}\left(\overline{\boldsymbol{K}} \cdot \boldsymbol{K}^{*}\right)\right]\right)\right. \\
& \left.-\frac{A}{f(A-f)} \exp \left[-\frac{A}{f(A-f)}\left(\overline{\boldsymbol{K}} \cdot \boldsymbol{K}^{*}\right)\right]\left(\left(\overline{\boldsymbol{K}} \cdot \boldsymbol{K}^{*}\right)\left(\overline{\boldsymbol{K}}_{12} \cdot \boldsymbol{K}_{12}^{*}\right)-\left(\overline{\boldsymbol{K}} \cdot \overline{\boldsymbol{K}}_{12}\right)\left(\boldsymbol{K}^{*} \cdot \boldsymbol{K}_{12}^{*}\right)\right)\right\} .
\end{aligned}
$$

This single-column BS transform is now in a form in which it can be combined with other single-column functions of this type to construct the BS transform for the full $A$-particle system. If this $1+3$-particle single-column transform is to be used in com- 
bination with other single-column functions, it is important to note that such an $H$ must contain the mass numbers $f$ and $A-f$ of the full $A$-particle system. The singlecolumn factors $\exp \eta\left(\overline{\boldsymbol{K}} \cdot \boldsymbol{K}^{*}\right)$ combine to make the simple factor $\exp \left(\overline{\boldsymbol{K}} \cdot \boldsymbol{K}^{*}\right)$ for the full 4-columned tableau; the only property of $\eta$ which is needed is $\Sigma_{\text {columns }} \eta=1$, see appendix $\mathrm{C}$.

Since such single-column BS transforms are the building blocks for the construction of many of the full BS transforms of this work, a catalogue of such single-column BS transforms is given in appendix C in a format similar to that of eq. (62). Such single-column BS transforms are particularly useful in heavier nuclei. In light nuclei a direct evaluation of the complete set of $y_{m\left(\lambda^{\prime} \mu^{\prime}\right)}$ and $D_{m}(p)$ for the full $A$-particle system is feasible and, through form II of the full BS transform, leads to the most direct evaluation of norm and overlap matrix elements. This method will therefore be illustrated by a more detailed example.

\section{A detailed example: $\mathrm{The}{ }^{6} \mathrm{Li}+{ }^{6} \mathbf{L i}$ cluster system}

The ${ }^{6} \mathrm{Li}+{ }^{6} \mathrm{Li}$ binary fragment system is chosen to illustrate the direct method for the evaluation of the full BS transform. Each ${ }^{6} \mathrm{Li}$ fragment is assumed to have space symmetry [42] (with $S=1, T=0$ ), and $\operatorname{SU}(3)$ symmetry $\left(\lambda_{f} \mu_{f}\right)=(20)$ with $I_{f}=0$ or 2. The possible space symmetries of the full 12-particle system are then limited to [4422], [4431] and [444]. The coupling of the fragment $\mathrm{SU}(3)$ symmetries $\left(\lambda_{f} \mu_{f}\right)$ $\times\left(\lambda_{A-f} \mu_{A-f}\right)=(20) \times(20)$ leads to the possibilities $\left(\lambda_{c} \mu_{c}\right)=(40),(02),(21)$. The possible $(\lambda \mu)$ are listed in table 2 . For relative motion functions fixed in their lowest

TABLE 2

Possible $(\lambda \mu)$ for the ${ }^{6} \mathrm{Li}+{ }^{6} \mathrm{Li}$ cluster functions

\begin{tabular}{cccccccc}
\hline$\left(\lambda_{\mathrm{c}} \mu_{\mathrm{c}}\right)$ & \multicolumn{7}{c}{$(\lambda \mu)$} \\
\hline$(40)$ & $(Q+4,0)$ & $(Q+2,1)$ & $(Q 2)$ & $(Q-2,3)$ & $(Q-4,4)$ & $(Q-1,1)$ & $(Q-2,0)$ \\
$(02)$ & & $(Q 2)$ & $(Q-2,3)$ & $(Q+1,0)$ & $(Q-1,1)$ & $(Q-3,2)$ \\
$(21)$ & & $(Q+2,1)$ & $(Q 2)$ & $(Q-2,3)$ &
\end{tabular}

$\mathscr{A}\left[\left[\varphi\left(\xi_{6}\right)^{(42)(20)} \times \phi\left(\xi_{6}^{\prime}\right)^{(42)](20)}\right]^{\left[4 k^{\prime} h^{\prime \prime}\right)\left(\lambda_{c} \mu_{c}\right)} \times \chi(R)^{(Q 0)}\right]^{\alpha \lambda \mu)}$.

Pauli-allowed oscillator excitation, $Q=4$, the possible Pauli-allowed $\left(\lambda_{c} \mu_{c}\right)$ values are uniquely determined by the 12-particle space symmetries:

(i) $\left(\lambda_{c} \mu_{c}\right)=(40)$ for $[444]$ space symmetry since this is the only $\left(\lambda_{c} \mu_{c}\right)$ value for which the coupling $\left(\lambda_{c} \mu_{c}\right) \times(40)$ can give a resultant $(\lambda \mu)=(04)$;

(ii) $\left(\lambda_{c} \mu_{c}\right)=(21)$ for [4431] symmetry since $\left(\lambda_{c} \mu_{c}\right) \times(40) \rightarrow(\lambda \mu)=(12)$ is possible only with $\left(\lambda_{c} \mu_{c}\right)=(21)$; and

(iii) $\left(\lambda_{c} \mu_{c}\right)=(02)$ for [4422] symmetry since $\left(\lambda_{c} \mu_{c}\right) \times(40) \rightarrow(\lambda \mu)=(20)$ is possible only with $\left(\lambda_{c} \mu_{c}\right)=(02)$. 
The zeros required for all other $(\lambda \mu)$ values for $Q=4$ furnish a strong test for the full $Q$-dependent expressions for the overlap matrix elements. For $Q>4$ all three $\left(\lambda_{c} \mu_{c}\right)$ values may be possible for some $(\lambda \mu)$ values. For this cluster system, made up of two identical fragments, however, $\lambda_{c}+\mu_{c}+Q$ must be an even integer, as in the case of the ${ }^{12} \mathrm{C}+{ }^{12} \mathrm{C}$ system. As a result the norm matrix becomes a $2 \times 2$ matrix only for states of positive parity, (even $Q),(\lambda \mu)=(Q 2)$; with $\left(\lambda_{c} \mu_{c}\right)=(40)$ and $(02)$.

\subsection{STATES WITH [4422] SPACE SYMMETRY, $\left(\lambda_{c} \mu_{c}\right)=\left(\lambda_{c}^{\prime} \mu_{c}^{\prime}\right)=(40)$}

The evaluation of the $y_{m\left(\lambda^{\prime} \mu^{\prime}\right)}$ and $D_{m}(p)$ through the function $\mathscr{H}_{\alpha_{c}, \alpha_{c}^{\prime}}^{[422](40)(40)}$ is simple, since the choice $\alpha_{c}=\alpha_{c}^{\prime}=\varepsilon_{c} \Lambda_{c} M_{A_{c}}=800,\left(n_{z} n_{x} n_{y}=400\right)$, automatically selects the states with $\left(\lambda_{c} \mu_{c}\right)=\left(\lambda_{c}^{\prime} \mu_{c}^{\prime}\right)=(40)$. The specific construction of such states involves differentiations with respect to four $k_{i_{z}}$ variables and differentiations with respect to four $k_{j_{z}}^{*}$ variables. The construction of states with space symmetry [4422] is illustrated in fig. $2 \mathrm{a}$. In fig. 2 , particles associated with the two different ${ }^{6} \mathrm{Li}$ fragments

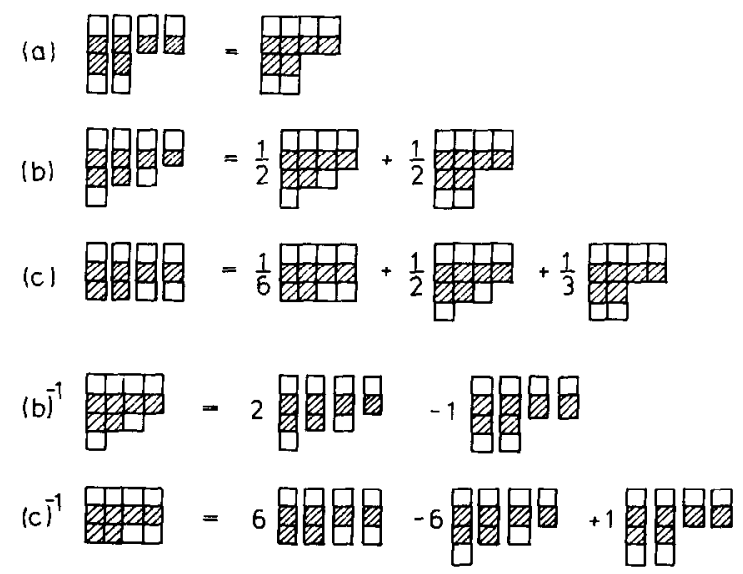

Fig. 2. The combination of single-column functions for the 6+6-particle system.

are illustrated by white and shaded squares. Initially, squares associated with the 1st and 2 nd rows are assumed to represent particles with 0 s excitations, squares associated with the 3 rd and 4 th rows $z$-excitations only, making the initial row symmetrization automatic. The separated columns denote the column antisymmetrizations associated with the definition of the $F($ : : :) functions of eq. (57). In general, such columns can couple to several resultant space symmetries. In fig. $2 \mathrm{a}$, however, this coupling is unique and gives the single state of [4422] symmetry. For example, the last two columns can couple only to space symmetry [22]. In general, two $\left[1^{2}\right]$ symmetries could couple via $\left[1^{2}\right] \times\left[1^{2}\right]=\left[1^{4}\right]+[211]+[22]$. States of symmetry $\left[1^{4}\right]$ and $[211]$, however, would correspond to states with at least two 0 s particles 
in the same fragment and in the same column, and would correspond to two 0s particles with identical spin-isospin quantum numbers in the same fragment. Thus

$\mathscr{H}_{800 ; 800}^{(4422)(40),(40)} \doteq\left[F\left(\begin{array}{c}0 \bar{z} ; 0 \bar{z} \\ \left.0 z^{*} ; 0 z^{*}\right)\end{array}\right]^{2}\left[F\left(\begin{array}{l}0 ; 0 \\ 0 ; 0\end{array}\right)\right]^{2}\right.$

$$
=\exp \left(\bar{K} \cdot K^{*}\right)\left[\left(1-e^{1}\right)^{2}-e^{1} \beta_{z z}^{2}\right]^{2}\left(1-e^{1}\right)^{2},
$$

where these $F$-functions have been read from table 9 . We use the shorthand notation of appendix D: With $f^{\prime}=f$ :

$$
e^{p} \equiv \exp \left\{-p \frac{A}{f(A-f)}\left(\bar{K} \cdot K^{*}\right)\right\}, \quad \beta_{z z} \equiv \frac{A}{f(A-f)} \bar{K}_{z} K_{z}^{*} .
$$

The symbol $\doteq$ is defined to mean "equal to within an overall multiplicative constant". This constant will always be absorbed by the as yet undetermined $y_{m\left(\lambda^{\prime} \mu^{\prime}\right)}$. For the general binary fragment system only the $p=0, m=0,\left(\lambda^{\prime} \mu^{\prime}\right)=\left(\lambda_{c} \mu_{c}\right)$ term can make a contribution to a norm matrix element of a completely separated 2-fragment system; (this corresponds to the limit $Q \rightarrow \infty$ ). With the $F$-functions of appendix D the normalization of eq. (49) automatically leads to $y_{0\left(\lambda_{c} \mu_{c}\right)}=1$. For a system of two identical fragments both the $p=0$ and the $p=f\left(\right.$ full exchange) $m=0,\left(\lambda^{\prime} \mu^{\prime}\right)=\left(\lambda_{c} \mu_{c}\right)$ terms make (equal) contributions to the $(Q \rightarrow \infty)$ norm matrix elements. In this special case, therefore, the product of $F$-functions must be renormalized by a factor of $\frac{1}{2}$. For $\left(\lambda_{c} \mu_{c}\right)=\left(\lambda_{c}^{\prime} \mu_{c}^{\prime}\right)=(40)$ the possible $m\left(\lambda^{\prime} \mu^{\prime}\right)$ values are given by

$$
m\left(\lambda^{\prime} \mu^{\prime}\right)=m(4-m, 0) .
$$

Thus

$$
\begin{aligned}
\frac{1}{2}\left[\left(1-e^{1}\right)^{2}-e^{1} \beta_{z z}^{2}\right]^{2} & \left(1-e^{1}\right)^{2}=\frac{1}{2}\left\{\left(1-e^{1}\right)^{6}-2 e^{1}\left(1-e^{1}\right)^{4} \beta_{z z}^{2}+e^{2}\left(1-e^{1}\right)^{2} \beta_{z z}^{4}\right\} \\
& =\sum_{m=0}^{4} y_{m(4-m, 0)} m !\left[\frac{A}{f(A-f)}\right]^{m}\left[\sum_{p} D_{m}(p) e^{p}\right] \sum_{\alpha, \beta} P(\bar{K})_{\beta}^{(m 0)} P\left(K^{*}\right)_{\alpha}^{(0 m)} \\
& \times \sum_{\gamma}\langle(m 0) \alpha ;(4-m, 0) \gamma \mid(40) 800\rangle\langle(m 0) \beta ;(4-m, 0) \gamma(40) 800\rangle
\end{aligned}
$$

The SU(3) $\supset \mathrm{SU}(2) \times \mathrm{U}(1)$ coupling rules define $\alpha, \beta$ and' $\gamma$ uniquely in this case: $\beta=\alpha \equiv \varepsilon_{\alpha} \Lambda_{\alpha} M_{A_{\alpha}}=2 m 00 ; \gamma=8-2 m 00$. For this unique coupling the $\operatorname{SU}(3)$ Wigner coefficients have the values +1 . With $P(\bar{K})_{2 m 00}^{(m 0)}=\bar{K}_{z}^{m} / \sqrt{ } m$ !, eq. (66) leads to: $y_{m(4-m, 0)}=0$ for $m=1,3$; and $y_{m(4-m, 0)}=1$ for $m=0,2,4$; with $D_{m}(p)$ given by simple binomial coefficients. These are tabulated in the first entry of table 3 .

\subsection{STATES WITH [4431] SPACE SYMMETRY, $\left(\lambda_{c} \mu_{c}\right)=\left(\lambda_{c}^{\prime} \mu_{c}^{\prime}\right)=(40)$}

States of [4431] symmetry can be constructed from the column antisymmetrizers illustrated in fig. $2 \mathrm{~b}$. The coupling of these columns is no longer unique. In particular, the coupling of the two $\left[1^{3}\right]$ symmetries corresponding to single column tableaux 


\section{TABLE 3}

Coefficients for the ${ }^{6} \mathrm{Li}+{ }^{6} \mathrm{Li}$ norms

\begin{tabular}{|c|c|c|c|}
\hline \multicolumn{2}{|c|}{$[f]=[4422]$} & \multicolumn{2}{|c|}{$\left(\lambda_{c} \mu_{c}\right)=(40),\left(\lambda_{c}^{\prime} \mu_{c}^{\prime}\right)=(40)$} \\
\hline$m$ & $\left(\lambda^{\prime} \mu^{\prime}\right)$ & $y_{m\left(\lambda^{\prime} \mu^{\prime}\right)}$ & $D_{m}(p)$ \\
\hline 0 & $(40)$ & 1 & $\frac{1}{2}(-1)^{p}\left(\begin{array}{c}6 \\
p\end{array}\right)$ \\
\hline 2 & $(20)$ & 1 & $(-1)^{p}\left({ }_{p-1}^{4}\right)$ \\
\hline 4 & $(00)$ & 1 & $\frac{1}{2}(-1)^{p}\left(p_{p}^{2}-2\right)$ \\
\hline \multicolumn{2}{|c|}{$[f]=[4422]$} & \multicolumn{2}{|c|}{$\left(\lambda_{c} \mu_{c}\right)=(40),\left(\lambda_{c}^{\prime} \mu_{c}^{\prime}\right)=(02)$} \\
\hline \multicolumn{4}{|c|}{$m=2$} \\
\hline \multicolumn{2}{|c|}{$[f]=[4422]$} & \multicolumn{2}{|c|}{$\left(\lambda_{c} \mu_{c}\right)=(02),\left(\lambda_{c}^{\prime} \mu_{c}^{\prime}\right)=(02)$} \\
\hline$m$ & $\left(\lambda^{\prime} \mu^{\prime}\right)$ & $y_{m\left(\lambda^{\prime} \mu^{\prime}\right)}$ & $D_{m}(p)$ \\
\hline 0 & $(02)$ & 1 & $\frac{1}{2}(-1)^{p}\left[\left({ }_{p}^{4}\right)-\left({ }_{p}{ }^{4} 1\right)+\left({ }_{p-2}{ }^{4}\right)\right]$ \\
\hline 1 & (11) & 6 & $\frac{1}{2}(-1)^{p}\left[\left({ }_{p-1}^{3}\right)-\left({ }_{p}^{3}-2\right)\right]$ \\
\hline 2 & $(20)$ & $\frac{1}{2}$ & $\frac{1}{2}(-1)^{p}\left[\left({ }_{p-1}^{2}\right)+20\left(p_{p-2}^{2}\right)+\left(p_{p-3}^{2}\right)\right]$ \\
\hline \multicolumn{2}{|c|}{$[f]=[4422]$} & \multicolumn{2}{|c|}{$\left(\lambda_{c} \mu_{c}\right)=(21),\left(\lambda_{c}^{\prime} \mu_{c}^{\prime}\right)=(21)$} \\
\hline$m$ & $\left(\lambda^{\prime} \mu^{\prime}\right)$ & $y_{m\left(\lambda^{\prime} \mu^{\prime}\right)}$ & $D_{m}(p)$ \\
\hline 0 & $(21)$ & 1 & $\frac{1}{2}(-1)^{p}\left[\left(\begin{array}{c}5 \\
p\end{array}\right)-\left(\begin{array}{c}5 \\
p-1\end{array}\right)\right]$ \\
\hline 1 & $(30)$ & $\frac{4}{3}$ & $(-1)^{p}\left(\begin{array}{c}4 \\
p-1\end{array}\right)$ \\
\hline & $(11)$ & $\frac{2}{3}$ & \\
\hline 2 & $(01)$ & 1 & $\frac{1}{2}(-1)^{p}\left[\left({ }_{p-1}^{3}\right)-\left({ }_{p-2}^{3}\right)\right]$ \\
\hline 3 & $(10)$ & $\frac{4}{3}$ & $(-1)^{p}\left(\begin{array}{c}2 \\
p-2\end{array}\right)$ \\
\hline
\end{tabular}


TABLE 3 (continued)

\begin{tabular}{|c|c|c|c|}
\hline \multicolumn{2}{|c|}{$[f]=[4431]$} & \multicolumn{2}{|c|}{$\left(\lambda_{c} \mu_{c}\right)=(40),\left(\lambda_{c}^{\prime} \mu_{c}^{\prime}\right)=(40)$} \\
\hline$m$ & $\left(\lambda^{\prime} \mu^{\prime}\right)$ & $y_{m\left(\lambda^{\prime} \mu^{\prime}\right)}$ & $D_{m}(p)$ \\
\hline 0 & $(40)$ & 1 & $\frac{1}{2}(-1)^{p}\left[\left(\begin{array}{l}5 \\
p\end{array}\right)-\left(\begin{array}{c}5 \\
p-1\end{array}\right)\right]$ \\
\hline 1 & $(30)$ & 1 & $2(-1)^{p}(p-1)$ \\
\hline 3 & (10) & 1 & $2(-1)^{p}\left({ }_{p-2}^{2}\right)$ \\
\hline 4 & $(00)$ & 1 & $-\frac{1}{2}(-1)^{p}\left[\left({ }_{p}^{1}-2\right)-\left({ }_{p-3}^{1}\right)\right]$ \\
\hline \multicolumn{4}{|c|}{$[f]=[4431]$} \\
\hline \multicolumn{2}{|c|}{$[f]=[4431]$} & \multicolumn{2}{|c|}{$\left(\lambda_{\mathrm{c}} \mu_{\mathrm{c}}\right)=(02),\left(\lambda_{\mathrm{c}}^{\prime} \mu_{\mathrm{c}}^{\prime}\right)=(02)$} \\
\hline$m$ & $\left(\lambda^{\prime} \mu^{\prime}\right)$ & $y_{m\left(\lambda^{\prime} \mu^{\prime}\right)}$ & $D_{m}(p)$ \\
\hline 0 & $(02)$ & 1 & $\frac{1}{2}(-1)^{p}\left[\left(\begin{array}{l}4 \\
p\end{array}\right)-\left({ }_{p-2}^{4}\right)\right]$ \\
\hline 1 & (11) & 4 & $\frac{1}{2}(-1)^{p}\left(p_{p-1}^{4}\right)$ \\
\hline \multicolumn{2}{|c|}{$[f]=[4431]$} & \multicolumn{2}{|c|}{$\left(\lambda_{c} \mu_{c}\right)=(21),\left(\lambda_{c}^{\prime} \mu_{c}^{\prime}\right)=(21)$} \\
\hline$m$ & $\left(\lambda^{\prime} \mu^{\prime}\right)$ & $y_{m\left(\lambda^{\prime} \mu^{\prime}\right)}$ & $D_{m}(p)$ \\
\hline 0 & (21) & 1 & $\frac{1}{2}(-1)^{p}\left[\left({ }_{p}^{4}\right)+\left(\begin{array}{c}4 \\
p-2\end{array}\right)\right]$ \\
\hline 1 & $\begin{array}{l}(30) \\
(11)\end{array}$ & $\begin{array}{l}\frac{4}{3} \\
\frac{8}{3}\end{array}$ & $\frac{1}{2}(-1)^{p}\left[\left({ }_{p-1}^{3}\right)-\left({ }_{p}^{3}-2\right)\right]$ \\
\hline 2 & $\begin{array}{l}(01) \\
(20)\end{array}$ & $\begin{array}{l}4 \\
4\end{array}$ & $\frac{1}{2}(-1)^{p}\left({ }_{p-2}^{2}\right)$ \\
\hline 3 & (10) & $\frac{4}{3}$ & $-\frac{1}{2}(-1)^{p}\left[\left({ }_{p-2}^{1}\right)-\left({ }_{p-3}^{1}\right)\right]$ \\
\hline
\end{tabular}


TABLE 3 (continued)

\begin{tabular}{|c|c|c|c|}
\hline \multicolumn{2}{|c|}{$[f]=[444]$} & \multicolumn{2}{|c|}{$\left(\lambda_{c} \mu_{c}\right)=(40),\left(\lambda_{c}^{\prime} \mu_{c}^{\prime}\right)=(40)$} \\
\hline$m$ & $\left(\lambda^{\prime} \mu^{\prime}\right)$ & $y_{m\left(\lambda^{\prime} \mu^{\prime}\right)}$ & $D_{m}(p)$ \\
\hline 0 & (40) & 1 & $\frac{1}{2}(-1)^{p}\left[\left(\begin{array}{l}4 \\
p\end{array}\right)-4\left({ }_{p-1}^{4}\right)+\left({ }_{p-2}^{4}\right)\right]$ \\
\hline 1 & (30) & 1 & $6(-1)^{p}\left[\left(p_{p-1}\right)-\left(p^{3}-2\right)\right]$ \\
\hline 2 & (20) & 1 & $-2(-1)^{p}\left[\left({ }_{p-1}^{2}\right)-7\left({ }_{p-2}^{2}\right)+\left({ }_{p-2}^{3}\right)\right]$ \\
\hline 3 & (10) & 1 & $-6(-1)^{p}\left[\left({ }_{p}^{1}-2\right)-\left(\begin{array}{c}1 \\
p-3\end{array}\right)\right]$ \\
\hline 4 & $(00)$ & 1 & $\frac{1}{2}(-1)^{p}\left[\left(\begin{array}{c}0 \\
p-2\end{array}\right)-4\left(\begin{array}{c}0 \\
p-3\end{array}\right)+\left(\begin{array}{c}0 \\
p-4\end{array}\right)\right]$ \\
\hline \multicolumn{2}{|c|}{$[f]=[444]$} & \multicolumn{2}{|c|}{$\left(\lambda_{c} \mu_{c}\right)=(40),\left(\lambda_{c}^{\prime} \mu_{c}^{\prime}\right)=(02)$} \\
\hline \multicolumn{2}{|c|}{$m=2:$} & 20) $=1$ & $D_{2}(p)=(-1)^{p}\left({ }_{p-1}^{4}\right)$ \\
\hline \multicolumn{2}{|c|}{$[f]=[444]$} & \multicolumn{2}{|c|}{$\left(\lambda_{c} \mu_{c}\right)=(02),\left(\lambda_{c}^{\prime} \mu_{c}^{\prime}\right)=(02)$} \\
\hline$m$ & $\left(\lambda^{\prime} \mu^{\prime}\right)$ & $y_{m\left(\lambda^{\prime} \mu^{\prime}\right)}$ & $D_{m}(p)$ \\
\hline 0 & (02) & 1 & $\frac{1}{2}(-1)^{p}\left(\begin{array}{c}6 \\
p\end{array}\right)$ \\
\hline 2 & (20) & 1 & $-\frac{1}{2}(-1)^{p}\left({ }_{p-1}^{4}\right)$ \\
\hline \multicolumn{2}{|c|}{$[f]=[444]$} & \multicolumn{2}{|c|}{$\left(\lambda_{r} \mu_{r}\right)=(21),\left(\lambda_{r}^{\prime} \mu_{r}^{\prime}\right)=(21)$} \\
\hline$m$ & $\left(\lambda^{\prime} \mu^{\prime}\right)$ & $y_{m\left(\lambda^{\prime} \mu^{\prime}\right)}$ & $D_{m}(p)$ \\
\hline 0 & (21) & 1 & $\frac{1}{2}(-1)^{p}\left[\left(\begin{array}{c}5 \\
p\end{array}\right)-\left({ }_{p-1}^{5}\right)\right]$ \\
\hline 1 & $\begin{array}{l}(30) \\
(11)\end{array}$ & $\begin{array}{l}\frac{4}{3} \\
-\frac{16}{3}\end{array}$ & $-\frac{1}{2}(-1)^{p}\left({ }_{p-1}^{4}\right)$ \\
\hline 2 & $\begin{array}{l}(01) \\
(20)\end{array}$ & $\begin{array}{l}2 \\
0\end{array}$ & $-\frac{1}{2}(-1)^{p}\left[\left({ }_{p}^{3}-1\right)-\left({ }_{p-2}^{3}\right)\right]$ \\
\hline 3 & (10) & $\frac{4}{3}$ & $-\frac{1}{2}(-1)^{p}(\underset{p-2}{2})$ \\
\hline
\end{tabular}


with the shadings of fig. $2 \mathrm{~b}$ is equivalent to the coupling, illustrated by the ket vectors to be associated with fig. $2 b$,

$$
\begin{aligned}
& \left|\square a_{1}\right\rangle\left|\square \alpha_{2}\right\rangle=\frac{1}{\sqrt{2}} \mid \square \\
& \left.\equiv|\square| \alpha_{1}\right\rangle\left|\square \alpha_{3}\right\rangle=\frac{1}{\sqrt{2}}|\square \alpha\rangle+\frac{1}{\sqrt{2}}|\square \alpha\rangle
\end{aligned}
$$

Such couplings can be effected by symmetric group or SU(4) coupling techniques. However, the equivalence with simple 2-rowed couplings, illustrated by eq. (67b), reduces all coupling coefficients needed for fig. 2 to simple SU(2) Wigner coefficients. The coupling coefficients used in eq. (67b) are $\left\langle\frac{1}{2} \frac{1}{2} \frac{1}{2}-\frac{1}{2} \mid S 0\right\rangle$, with $S=1$ and 0 for the first and second symmetries of eq. (67b). The additional columns of length 4 and 2 lead only to unique couplings. The coupling for the bra vectors associated with fig. $2 \mathrm{~b}$ are identical to those for the ket vectors. For the unit operator cross terms between different symmetries must be zero, leading to the simple matrix element relation illustrated by fig. $2 b$. From the inverse of this relation, fig. $2 b^{-1}$,

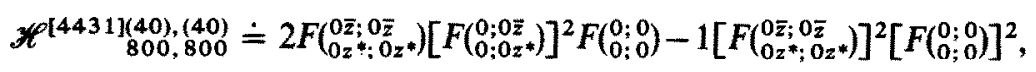

or

$$
\begin{array}{r}
\frac{1}{2}\left\{2\left[\left(1-e^{1}\right)^{2}-e^{1} \beta_{z z}^{2}\right]\left[\left(1-e^{1}\right)-e^{1} \beta_{z z}\right]^{2}\left(1-e^{1}\right)-\left[\left(1-e^{1}\right)^{2}-e^{1} \beta_{z z}^{2}\right]^{2}\left(1-e^{1}\right)^{2}\right\} \\
=\sum_{m=0}^{4} y_{m(4-m, 0)}\left[\sum_{p} D_{m}(p) e^{p}\right]\left[\frac{A}{f(A-f)}\right]^{m} \bar{K}_{z}^{m} K_{z}^{* m},
\end{array}
$$

leading at once to the determination of the $y_{m(4-m, 0)}$ and $D_{m}(p)$. These are listed in table 3.

\subsection{STATES WITH [444] SPACE SYMMETRY, $\left(\lambda_{c} \mu_{c}\right)=\left(\lambda_{c}^{\prime} \mu_{c}^{\prime}\right)=(40)$}

The coupling coefficients associated with fig. $2 \mathrm{c}$ are equivalent to $\langle 111-1 \mid S 0\rangle$ with $S=2,1,0$. The relation, illustrated by fig. $2 \mathrm{c}^{-1}$, leads to

$$
\begin{aligned}
\frac{1}{2}\left\{6\left[\left(1-e^{1}\right)-e^{1} \beta_{z z}\right]^{4}\right. & -6\left[\left(1-e^{1}\right)^{2}-e^{1} \beta_{z z}^{2}\right]\left[\left(1-e^{1}\right)-e^{1} \beta_{z z}\right]^{2}\left(1-e^{1}\right) \\
+ & \left.1\left[\left(1-e^{1}\right)^{2}-e^{1} \beta_{z z}^{2}\right]^{2}\left(1-e^{1}\right)^{2}\right\} \\
& =\sum_{m=0}^{4} y_{m(4-m, 0)}\left[\sum_{p} D_{m}(p) e^{p}\right]\left[\frac{A}{f(A-f)}\right]^{m} K_{z}^{m} K_{z}^{* m} .
\end{aligned}
$$

4.4. MATRIX ELEMENTS WITH $\left(\lambda_{c} \mu_{c}\right)=(40),\left(\lambda_{c}^{\prime} \mu_{c}^{\prime}\right)=(21)$; [4422] SPACE SYMMETRY

From the identity of the two ${ }^{6} \mathrm{Li}$ fragments, matrix elements of this type must be 
zero. It is instructive to demonstrate this by explicit calculation. The calculation of $\mathscr{H}^{[4422](40),(21)}$, (again using the simplest possible values of $\alpha_{c}, \alpha_{c}^{\prime}$ ) is achieved by simple $\mathrm{SU}(3)$ couplings

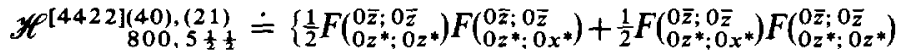

$$
\begin{aligned}
& \left.-\frac{1}{2} F\left(\begin{array}{c}
0 \bar{z} ; 0 \bar{z} \\
0 z^{*} ; 0 z^{*}
\end{array}\right) F\left(\begin{array}{c}
0 \bar{z} ; 0 \bar{z} \\
0 x^{*} ; 0 z^{*}
\end{array}\right)-\frac{1}{2} F\left(\begin{array}{c}
0 \bar{z} ; 0 \bar{z} \\
0 x^{*} ; 0 z^{*}
\end{array}\right) F\left(\begin{array}{c}
0 \bar{z} ; 0 \bar{z} \\
0 z^{*} ; 0 z^{*}
\end{array}\right)\right\}\left[F\left(\begin{array}{c}
0 ; 0 \\
0 ; 0
\end{array}\right)\right]^{2} \\
& =F\left(\begin{array}{c}
0 \bar{z} ; 0 \\
0 z^{*} ; 0 z^{*}
\end{array}\right)\left[F\left(\begin{array}{c}
0 \overline{0} ; 0 \bar{z} \\
0 z^{*} ; 0 x^{*}
\end{array}\right)-F\left(\begin{array}{c}
0 \bar{z} ; 0, \bar{z} \\
0 x^{*} ; 0 z^{*}
\end{array}\right)\right]\left[F\left(\begin{array}{c}
0 ; 0 \\
0 ; 0
\end{array}\right)\right]^{2}=0 \text {, }
\end{aligned}
$$

where we have used:

(i) $\left\langle(10) 200 ;(10)-1 \frac{1}{2} \frac{1}{2} \mid(20) 1 \frac{1}{2} \frac{1}{2}\right\rangle=\left\langle(10)-1 \frac{1}{2} \frac{1}{2} ;(10) 200 \mid(20) 1 \frac{1}{2} \frac{1}{2}\right\rangle=\sqrt{\frac{1}{2}}$ in com-

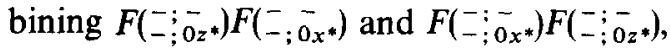

(ii) the commutativity of products of $F$-functions, and

(iii) $\left\langle(20) 400 ;(20) 1 \frac{1}{2} \frac{1}{2} \mid(21) 5 \frac{1}{2} \frac{1}{2}\right\rangle=-\left\langle(20) 1 \frac{1}{2} \frac{1}{2} ;(20) 400 \mid(21) 5 \frac{1}{2} \frac{1}{2}\right\rangle=\sqrt{\frac{1}{2}}$ in combining $F\left(\begin{array}{c}-;-; \\ 0 z^{*} ; 0 z^{*}\end{array}\right) F\left(\begin{array}{c}-;-; \\ 0 z^{*} ; 0 x^{*}\end{array}\right)$ with $F\left(\begin{array}{c}-i-i \\ 0 z^{*} ; 0 z^{*}\end{array}\right) F\left(0 x^{*} ; 0 z^{*}\right)$, and

(iv) the identity of the $F$-functions in the square bracket of eq. (71) (see appendix D).

\subsection{MATRIX ELEMENTS FOR STATES WITH [4422] SPACE SYMMETRY, $\left(\lambda_{c} \mu_{c}\right)=\left(\lambda_{c}^{\prime} \mu_{c}^{\prime}\right)=(21)$}

For the states of lower $\mathrm{SU}(3)$ symmetries it will be advantageous to use the fact that the $\mathscr{H}^{[h]\left(\lambda_{c} \mu_{c}\right)\left(\lambda_{c}^{\prime} \mu_{c}^{\prime}\right)}$ for higher $\mathrm{SU}(3)$ symmetries are known from earlier steps in the process of calculation.

By simple SU(3) couplings

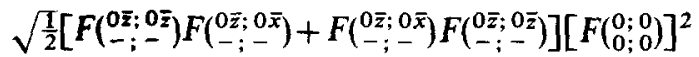

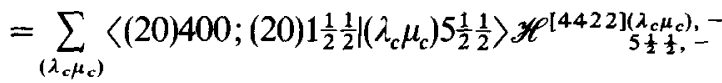

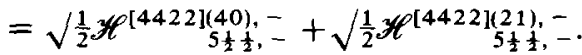

Similarly

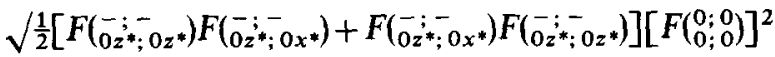

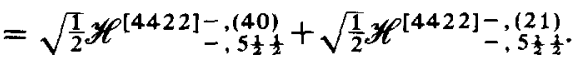

Combining eqs. (72) and (73) leads to

$$
\begin{aligned}
& \frac{2}{2}\left[F\left(\begin{array}{c}
0 \bar{z} ; 0 \bar{z} \\
0 z^{*} ; 0 z^{*}
\end{array}\right) F\left(\begin{array}{c}
0 \overline{0} ; 0 \bar{x} \\
0 z^{*} ; 0 x^{*}
\end{array}\right)+F\left(\begin{array}{c}
0 \bar{z} ; 0 \bar{z} \\
0 z^{*} ; 0 x^{*}
\end{array}\right) F\left(\begin{array}{c}
0 \bar{z} ; 0 \bar{x} \\
0 z^{*} ; 0 z^{*}
\end{array}\right)\right]\left[F\left(\begin{array}{c}
0 ; 0 \\
0 ; 0
\end{array}\right)\right]^{2}
\end{aligned}
$$

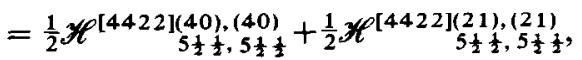

where we have used the result of $4.4, \mathscr{H}_{\alpha_{0}, \alpha_{*}^{(h)}}^{[h](40),(21)}=0 . \mathscr{H}^{[4422](40),(40)}$ can now be expanded by means of SU(3) Wigner coefficients $\left\langle(m 0) \alpha ;(4-m, 0) \gamma \mid(40) 5 \frac{1}{2} \frac{1}{2}\right\rangle$ in terms of the known $y_{m(4-m, 0)}$ and $D_{m}(p)$, calculated for $[h]=[4422],\left(\lambda_{c} \mu_{c}\right)=\left(\lambda_{c}^{\prime} \mu_{c}^{\prime}\right)=$ 
(40) in 4.1. Thus eq. (74) can be solved for $\mathscr{H}^{[4422](21),(21)} \sum_{\frac{1}{2} \frac{1}{2}, 5 \frac{1}{2}}^{\left(\frac{1}{2}\right)}$ to give

$$
\begin{aligned}
& \left\{\left[\left(1-e^{1}\right)^{2}-e^{1} \beta_{z z}^{2}\right]\left[\left(1-e^{1}\right)-e^{1}\left(\beta_{z z}+\beta_{x x}+\beta_{z z} \beta_{x x}\right)\right]+e^{2} \beta_{x z} \beta_{z x} \beta_{z z}^{2}\right\}\left(1-e^{1}\right)^{2} \\
& -\frac{1}{2}\left\{\frac{1}{2}\left(1-e^{1}\right)^{6}-e^{1}\left(1-e^{1}\right)^{4}\left[\left\langle(20) 400 ;(20) 1 \frac{1}{2} \frac{1}{2} \mid(40) 5 \frac{1}{2} \frac{1}{2}\right\rangle^{2} \beta_{z z}^{2}\right.\right. \\
& \left.\left.+\left\langle(20) 1 \frac{1}{2} \frac{1}{2} ;(20) 400 ; \mid(40) 5 \frac{1}{2} \frac{1}{2}\right\rangle^{2} 2 ! \beta_{z z} \beta_{x x}\right]+\frac{1}{2} e^{2}\left(1-e^{1}\right)^{2} 4 ! \beta_{x x} \beta_{z z}^{3} / 3 !\right\} \\
& =\frac{1}{2} \mathscr{H}^{[4422](21),(21)} \\
& =\frac{1}{2} \sum_{m\left(\lambda^{\prime} \mu^{\prime}\right)} y_{m\left(\lambda^{\prime} \mu^{\prime}\right)} m !\left[\frac{A}{f(A-f)}\right]^{m}\left[\sum_{p} e^{p} D_{m}(p)\right] \\
& \times \sum_{\alpha} P(\bar{K})_{\alpha}^{(m 0)} P\left(K^{*}\right)_{\alpha}^{(0 m)} \sum_{\gamma}\left\langle(m 0) \alpha ;\left(\lambda^{\prime} \mu^{\prime}\right) \gamma \mid(21) 5 \frac{1}{2} \frac{1}{2}\right\rangle^{2},
\end{aligned}
$$

\begin{tabular}{|c|c|c|c|}
\hline \multicolumn{2}{|c|}{$[f]=[444]$} & \multicolumn{2}{|c|}{$\left(\lambda_{c} \mu_{c}\right)_{4+8}=(40),\left(\lambda_{c} \mu_{c}\right)_{6+6}=(40)$} \\
\hline$m$ & $\left(\lambda^{\prime} \mu^{\prime}\right)$ & $y_{m\left(\lambda^{\prime} \mu^{\prime}\right)}$ & $D_{m}(p)$ \\
\hline $\begin{array}{l}0 \\
1 \\
2 \\
3 \\
4\end{array}$ & $\begin{array}{l}(40) \\
(30) \\
(20) \\
(10) \\
(00)\end{array}$ & $\begin{array}{l}\sqrt{3} \\
\sqrt{3} \\
\sqrt{3} \\
\sqrt{3} \\
\sqrt{3}\end{array}$ & $\begin{array}{l}(-1)^{p}\left(\begin{array}{l}4 \\
p\end{array}\right) \\
-2(-1)^{p}\left[\left(\begin{array}{l}3 \\
p\end{array}\right)-\left(\begin{array}{c}3 \\
p-1\end{array}\right)\right] \\
(-1)^{p}\left[\left(\begin{array}{l}2 \\
p\end{array}\right)-4\left(\begin{array}{c}2 \\
p-1\end{array}\right)+\left({ }_{p-2}^{2}\right)\right] \\
2(-1)^{p}\left[\left(\begin{array}{c}1 \\
\left.(p-1)-\left(\begin{array}{c}1 \\
p-2\end{array}\right)\right] \\
\left(\begin{array}{c}0 \\
p-2\end{array}\right)\end{array}\right.\right.\end{array}$ \\
\hline \multicolumn{2}{|c|}{$[f]=[444]$} & \multicolumn{2}{|c|}{$\left(\lambda_{c} \mu_{c}\right)_{4+8}=(40),\left(\lambda_{c} \mu_{c}\right)_{6+6}=(21)$} \\
\hline$m$ & $\left(\lambda^{\prime} \mu^{\prime}\right)$ & $y_{m\left(\lambda^{\prime} \mu^{\prime}\right)}$ & $D_{m}(p)$ \\
\hline $\begin{array}{l}1 \\
2 \\
3\end{array}$ & $\begin{array}{l}(30) \\
(20) \\
(10)\end{array}$ & $\begin{array}{l}2 \\
\sqrt{3} \\
2\end{array}$ & $\begin{array}{l}(-1)^{p}\left(\begin{array}{l}4 \\
p\end{array}\right) \\
-(-1)^{p}\left[\left(\begin{array}{l}3 \\
p\end{array}\right)-\left(\begin{array}{c}3 \\
p-1\end{array}\right)\right] \\
-(-1)^{p}\left(\begin{array}{l}2 \\
p-1\end{array}\right)\end{array}$ \\
\hline \multicolumn{2}{|c|}{$[f]=[444]$} & \multicolumn{2}{|c|}{$\left(\lambda_{c} \mu_{c}\right)_{4+8}=(40),\left(\lambda_{c} \mu_{c}\right)_{6+6}=(02)$} \\
\hline & & $y_{2(20)}=$ & $D_{2}(p)=(-1)^{p}\left(\begin{array}{l}4 \\
p\end{array}\right)$ \\
\hline
\end{tabular}

where, for $\left(\lambda_{c} \mu_{c}\right)=\left(\lambda_{c}^{\prime} \mu_{c}^{\prime}\right)==(21)$, the $m\left(\lambda^{\prime} \mu^{\prime}\right)$ values in the sum are restricted to the

TABLE 4

Coefficients for the ${ }^{6} \mathrm{Li}+{ }^{6} \mathrm{Li}-\alpha+{ }^{8} \mathrm{Be}$ overlaps 
possibilities:

$$
\begin{array}{ll}
m=0, & \left(\lambda^{\prime} \mu^{\prime}\right)=(21), \quad m=1, \quad\left(\lambda^{\prime} \mu^{\prime}\right)=(30),(11), \\
m=2, & \left(\lambda^{\prime} \mu^{\prime}\right)=(20),(01), \quad m=3, \quad\left(\lambda^{\prime} \mu^{\prime}\right)=(10) .
\end{array}
$$

With a few simple SU(3) Wigner coefficients, eq. (75) leads at once to $y_{m\left(\lambda^{\prime} \mu^{\prime}\right)}$ and $D_{m}(p)$ for these new quantum numbers. (The results are tabulated in table 3 ).

Exactly the same technique can be used for the overlaps between cluster functions involving different fragment decompositions $\left(f^{\prime} \neq f\right)$. The needed $F$-functions are included in appendix D. As an illustration, table 4 gives the coefficients needed to calculate the overlaps between the cluster functions ${ }^{6} \mathrm{Li}+{ }^{6} \mathrm{Li}$ and $\alpha+{ }^{8} \mathrm{Be}$ by means of eq. (51) or (52).

\section{Norms for simple binary fragment systems}

The BS transform in form II lcads to a very direct evaluation of norm and overlap matrix elements. In heavier nuclei, however, the total number of structure and exchange coefficients can be large so that the direct method based on eq. (51) can become somewhat cumbersome. In this case it is easier to calculate the full BS transform for the $A$-particle system from suitably symmetrized combinations of single-column transforms of the type illustrated in scct. 2 with the $1+3$-particle example. In this method it is advantageous to express the single-column transforms in terms of scalar products of $\overline{\boldsymbol{K}}$ vectors with $\boldsymbol{K}^{*}$ vectors, or $\left[\overline{\boldsymbol{K}}_{a} \times \overline{\boldsymbol{K}}_{b}\right]$ vectors, as in eq. (62). A fairly complete catalogue of such single-column functions is given in appendix $C$.

The combination of such single-column functions to construct the full BS transform for the $A$-particle system of proper space symmetry $\left(\lambda_{c} \mu_{c}\right)$, and $\left(\lambda_{c}^{\prime} \mu_{c}^{\prime}\right)$ is particularly simple for the case of the binary fragment cluster decomposition for which one of the fragments has 0s internal excitations only; e.g. if the light fragment has mass number $f \leqq 4$. In this case $\left(\lambda_{c} \mu_{c}\right)=\left(\lambda_{c}^{\prime} \mu_{c}^{\prime}\right)$ is determined solely by the heavy fragment. There is a single overlap integral which gives the norm of such a cluster function. For simplicity it will be assumed that the heavy fragment has highest possible space symmetry and highest possible $\mathrm{SU}(3)$ symmetry; but this is not essential.

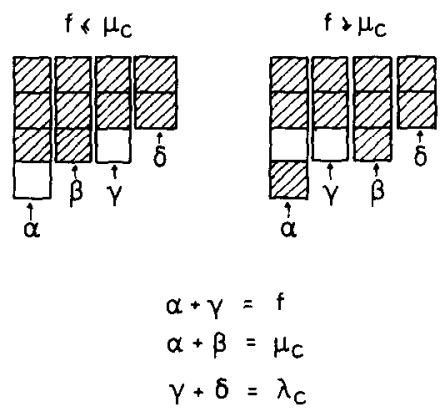

Fig. 3. Single-column functions for the binary fragment system with $8 \leqslant A-f \leqslant 12$. 
The case $8 \leqq A-f \leqq 12, f \leqq 4$, will be chosen as an illustrative example. It will be assumed that the heavy fragment has space symmetry $\left[h_{1} h_{2} h_{3}\right]=[44(A-$ $f-8)]$ and $\mathrm{SU}(3)$ symmetry $\left(\lambda_{c} \mu_{c}\right)=\left(4-\mu_{c}, \mu_{c}\right)$ with $\mu_{c}=A-f-8$. The full BS transform for this case will in general require a combination of the following singlecolumn transforms; (see fig. 3 and appendix C):

(i) $\alpha 1+3$-particle transforms with SU(3) symmetry (01) with internal excitations specified by the pseudo vector $\overline{\boldsymbol{K}}_{12}$, eq. (62);

(ii) $\beta 0+3$-particle transforms, also with $\mathrm{SU}(3)$ quantum numbers (01);

(iii) $\gamma 1+2$-particle transforms with internal excitations of SU(3) symmetry (10), carried by the vector $\overline{\boldsymbol{K}}_{1}$;

(iv) $\delta 0+2$-particle transforms, also with SU(3) symmetry (10).

$$
\begin{aligned}
& S_{\alpha \beta \gamma \delta}=\exp \left(\overline{\boldsymbol{K}} \cdot \boldsymbol{K}^{*}\right)\left\{\left[\left(1-e^{1}\right)\left(\overline{\boldsymbol{K}}_{12} \cdot \boldsymbol{K}_{12}^{*}\right)-\frac{A}{f(A-f)} e^{1}\left[\left(\overline{\boldsymbol{K}} \cdot \boldsymbol{K}^{*}\right)\left(\overline{\boldsymbol{K}}_{12} \cdot \boldsymbol{K}_{12}^{*}\right)\right.\right.\right. \\
& \left.\left.-\left(\overline{\boldsymbol{K}} \cdot \overline{\boldsymbol{K}}_{12}\right)\left(\boldsymbol{K}^{*} \cdot \boldsymbol{K}_{12}^{*}\right)\right]\right]^{\alpha}\left(\overline{\boldsymbol{K}}_{12} \cdot \boldsymbol{K}_{12}^{*}\right)^{\beta}\left[\left(1-e^{1}\right)\left(\overline{\boldsymbol{K}}_{1} \cdot \boldsymbol{K}_{1}^{*}\right)-\frac{A}{f(A-f)} e^{1}\left(\overline{\boldsymbol{K}} \cdot \boldsymbol{K}_{1}^{*}\right)\left(\overline{\boldsymbol{K}}_{1} \cdot \boldsymbol{K}^{*}\right)\right]^{p} \\
& \left.\times\left(\overline{\boldsymbol{K}}_{1} \cdot \boldsymbol{K}_{1}^{*}\right)^{\delta}\right\},
\end{aligned}
$$

The symmetric product of these single-column transforms, $S_{\alpha \beta \gamma \delta}$, with $\alpha$ and $\beta$ factors coupled symmetrically to $\left(0 \mu_{c}\right), \gamma$ and $\delta$ factors coupled symmetrically to $\left(\lambda_{c} 0\right)$, and the resultant coupled to $\left(\lambda_{\mathrm{c}} \mu_{\mathrm{c}}\right)$, with $\bar{K}_{1}$ carrying a $(40)$ excitation, and $\bar{K}_{2}$ carrying a $\left(\mu_{c} 0\right)$ excitation, has the desired SU(3) symmetry. But, $S_{\alpha \beta \gamma \delta}$ will in general be equal to a linear combination of several space symmetries, (cf. the discussion of subsects. 4.1, 4.2 and 4.3);

(ia) With $f \leqq \mu_{c}$ and $\gamma=0$, the 4 single-column functions of $S_{f\left(\mu_{c}-f\right) 0 \lambda_{c}}$ couple uniquely to space symmetry $\left[44 \mu_{c} f\right]$.

(ib) With $f \geqq \mu_{c}, \beta=0$, the 4 single-column functions of $S_{\mu_{c} 0\left(f-\mu_{c}\right)(4-f)}$ couple uniquely to space symmetry $\left[44 f \mu_{c}\right]$.

(iia) With $f \leqq \mu_{c}$, and $\gamma=1$

$$
S_{(f-1)\left(\mu_{c}-f+1\right) 1\left(\lambda_{c}-1\right)}=\frac{1}{\mu_{c}+2-f} H^{\left[44 \mu_{c}+1 f-1\right]}+\frac{\mu_{c}+1-f}{\mu_{c}+2-f} H^{\left[44 \mu_{c} f\right]} .
$$

This is the generalization of case (b) of fig. 2 , with coefficients

$$
\left\langle\frac{1}{2}\left(\mu_{c}+1-f\right)^{\frac{1}{2}}\left(\mu_{c}+1-f\right) \frac{1}{2}-\frac{1}{2} \mid S \frac{1}{2}\left(\mu_{c}-f\right)\right\rangle^{2} .
$$

Hence

$$
H^{\left[44 \mu_{c}+1 f-1\right]}=\left(\mu_{c}+2-f\right) S_{(f-1)\left(\mu_{c}-f+1\right) 1\left(\lambda_{c}-1\right)}-\left(\mu_{c}+1-f\right) S_{f\left(\mu_{c}-f\right) 0 \lambda_{c}^{*}}
$$

Similarly:

(iib) With $f \geqq \mu_{c}$; and $\beta=1$,

$$
H^{\left[44 f+1 \mu_{c}-1\right]}=\left(\mu_{c}+2-f\right) S_{\left(\mu_{c}-1\right) 1\left(f+1-\mu_{c}\right)(3-f)}-\left(\mu_{c}+1-f\right) S_{\mu_{c} 0\left(f-\mu_{c}\right)(4-f)}
$$


The only other case possible is:

(iii) $f=\mu_{c}=2$. This is equivalent to the case illustrated by fig. $2 \mathrm{c}$ and gives

$$
H^{[444]}=6 S_{0220}-6 S_{1111}+1 S_{2002} \text {. }
$$

To transform $H\left(\bar{K}, K^{*}\right)$ into the desired form, eq. (29), it is uscful to cxpand $S_{\alpha \beta \gamma \delta}$. Binomial expansions of the $\alpha$ and $\gamma$ factors, followed by expansions of the exponentials, give

$$
\begin{aligned}
& S_{\alpha \beta \gamma \delta}=\sum_{Q=m+l l, m, n} \sum_{p}\left(\begin{array}{l}
\gamma \\
l
\end{array}\right)\left(\begin{array}{l}
\alpha \\
m
\end{array}\right)\left(\begin{array}{l}
m \\
n
\end{array}\right)\left(\begin{array}{l}
f-l-m \\
p-l-m
\end{array}\right)(-1)^{p+n} \\
& \quad \times\left(\frac{A}{f(A-f)}\right)^{l+m}\left[1-p \frac{A}{f(A-f)}\right]^{Q-m-l} \frac{1}{(Q-m-l) !}\left(\overline{\boldsymbol{K}}_{1} \cdot \boldsymbol{K}^{*}\right)^{l}\left(\overline{\boldsymbol{K}} \cdot \boldsymbol{K}_{1}^{*}\right)^{l}\left(\overline{\boldsymbol{K}}_{1} \cdot \boldsymbol{K}_{1}^{*}\right)^{\lambda_{c}-l} \\
& \quad \times\left(\overline{\boldsymbol{K}} \cdot \overline{\boldsymbol{K}}_{12}\right)^{n}\left(\boldsymbol{K}^{*} \cdot \boldsymbol{K}_{12}^{*}\right)^{n}\left(\overline{\boldsymbol{K}}_{12} \cdot \boldsymbol{K}_{12}^{*}\right)^{\mu_{c}-n}\left(\overline{\boldsymbol{K}} \cdot \boldsymbol{K}^{*}\right)^{Q-n-l} .
\end{aligned}
$$

The combination of $P\left(\bar{K}_{1}\right)^{\left(\lambda_{c}-l, 0\right)}, P\left(\bar{K}_{1}\right)^{(l 0)}, \ldots$, to gain the desired final form, eq. (29), by $\mathrm{SU}(3)$ recoupling techniques can be achieved through a few key subsidiary formulae which are tabulated in subsect. B.2, and derived in subsect. B.4. Eq. (B.7) gives

$$
\begin{aligned}
\left.\left(\overline{\boldsymbol{K}}_{1} \cdot \boldsymbol{K}^{*}\right)^{l}\left(\overline{\boldsymbol{K}} \cdot \boldsymbol{K}_{1}^{*}\right)^{l}\left(\overline{\boldsymbol{K}}_{1} \cdot \boldsymbol{K}_{1}^{*}\right)\right)^{\lambda_{c}-l}=\sum_{(\bar{\lambda} \bar{\mu})}(l !)^{2} \frac{\left(\lambda_{c}-\bar{\mu}\right) !}{(l-\bar{\mu}) !}(-1)^{\lambda_{c}+l+\bar{\lambda}+\bar{\mu}}[d(\bar{\lambda} \bar{\mu})]^{\frac{1}{2}} \\
\times\left[\left[P\left(\overline{\boldsymbol{K}}_{1}\right)^{\left(\lambda_{c} 0\right)} \times P(\overline{\boldsymbol{K}})^{(l 0)}\right]^{(\bar{\lambda} \bar{\mu})} \times\left[P\left(\boldsymbol{K}_{1}^{*}\right)^{\left(0 \lambda_{c}\right)} \times P\left(\boldsymbol{K}^{*}\right)^{(0 l)}\right]^{(\bar{\mu} \bar{\lambda})}\right]_{00}^{(00)},
\end{aligned}
$$

where the phase factor comes from an additional interchange in the order of the $(0 l) \times$ $\left(0 \lambda_{c}\right) \rightarrow(\bar{\mu} \bar{\lambda})$ coupling, see eq. (A.3b). Similarly, eq. (B.9) gives

$$
\begin{aligned}
\left(\overline{\boldsymbol{K}} \cdot \overline{\boldsymbol{K}}_{12}\right)^{n}\left(\boldsymbol{K}^{*} \cdot \boldsymbol{K}_{12}^{*}\right)^{n}\left(\overline{\boldsymbol{K}}_{12} \cdot \boldsymbol{K}_{12}^{*}\right)^{\mu_{c}-n}\left(\overline{\boldsymbol{K}} \cdot \boldsymbol{K}^{*}\right)^{Q-n-1} \\
=\sum_{(\bar{\lambda} \bar{\mu})}\left(\mu_{c}-n\right) !(Q-n-l) ! \frac{\left(\mu_{c}-\bar{\mu}\right) !}{\left(\mu_{c}-n-\bar{\mu}\right) !} \frac{\left(\bar{\lambda}+\mu_{c}+2\right) !}{\left(\bar{\lambda}+\mu_{c}-n+2\right) !}[d(\bar{\lambda} \bar{\mu})]^{\frac{1}{2}} \\
\times\left[\left[P\left(\overline{\boldsymbol{K}}_{12}\right)^{\left(0 \mu_{c}\right)} \times P(\overline{\boldsymbol{K}})^{(Q-l, 0)}\right]^{(\overline{\bar{\lambda}} \overline{\bar{\mu}})} \times\left[P\left(\boldsymbol{K}_{12}^{*}\right)^{\left(\mu_{c} 0\right)} \times P\left(\boldsymbol{K}^{*}\right)^{(0, Q-l)}\right]^{(\overline{\bar{\mu}} \overline{\bar{\lambda}})}\right]_{00}^{(00)} .
\end{aligned}
$$

The combination of the $K$-space functions of eqs. (82) and (83) by the trivial 9-( $\lambda \mu$ ) transformation with three (00) representations, [cq. (A.16)], followed by the $9-(\lambda \mu)$ transformation

$$
\begin{aligned}
& {\left[\left[P\left(\overline{\boldsymbol{K}}_{1}\right)^{\left(\lambda_{c} 0\right)} \times P(\overline{\boldsymbol{K}})^{(l 0)}\right]^{(\bar{\lambda} \bar{\mu})} \times\left[P\left(\overline{\boldsymbol{K}}_{12}\right)^{\left(0 \mu_{c}\right)} \times P(\overline{\boldsymbol{K}})^{(Q-l, 0)}\right]^{(\bar{\lambda} \bar{\mu})}\right]_{\alpha}^{(\lambda \mu) \rho} } \\
&=\sum_{\left(\lambda_{c} \mu_{c}\right)}\left[\begin{array}{llll}
\left(\lambda_{c} 0\right) & (l 0) & (\bar{\lambda} \bar{\mu}) & - \\
\left(0 \mu_{c}\right) & (Q-l, 0) & (\bar{\lambda} \bar{\mu}) & - \\
\left(\lambda_{c} \mu_{c}\right) & (Q 0) & (\lambda \mu) & - \\
- & - & \rho
\end{array}\right] {\left[\begin{array}{l}
Q ! \\
l !(Q-l) !
\end{array}\right]^{\frac{1}{2}}\left[\left[P\left(\overline{\boldsymbol{K}}_{1}\right)^{\left(\lambda_{c} 0\right)}\right.\right.} \\
&\left.\left.\times P\left(\overline{\boldsymbol{K}}_{12}\right)^{\left(0 \mu_{c}\right)}\right]^{\left(\lambda_{c} \mu_{c}\right)} \times P(\overline{\boldsymbol{K}})^{(Q 0)}\right]_{\alpha}^{(\lambda \mu)},
\end{aligned}
$$


and the analogous 9-( $\lambda \mu)$ transformation in the $K^{*}$ functions, using (A.19), gives the desired combinations of SU(3) coupled $K$-space functions. Transformation to the $[(\bar{\lambda} \bar{\mu}) \times(\bar{\lambda} \bar{\mu})](\lambda \mu) \rho$ scheme via (A.16), in the first step, introduces a multiplicity label $\rho$ in the most general case. [The $\rho$-dependent phase factor introduced by (A.16) is canceled by the $\rho$-dependent phase factor of (A.19).]

The combination of all recoupling transformations gives

$$
\begin{aligned}
& S_{\alpha \beta \gamma \delta}=\sum_{Q} \sum_{(\lambda \mu) \rho} \sum_{l, m, n} \sum_{p}\left(\begin{array}{l}
l \\
l
\end{array}\right)\left(\begin{array}{l}
\alpha \\
m
\end{array}\right)\left(\begin{array}{l}
m \\
n
\end{array}\right)\left(\begin{array}{l}
f-l-m \\
p-l-m
\end{array}\right)(-1)^{p+n}\left(\frac{A}{f(A-f)}\right)^{l+m} \\
& \times\left[1-p \frac{A}{f(A-f)}\right]^{Q-m-l} \frac{Q !}{(Q-m-l) !(Q-l) !} \sum_{(\bar{\lambda} \bar{\mu})(\overline{\bar{\mu}} \overline{\bar{\mu}})}(-1)^{\lambda_{\mathrm{c}}+l+\bar{\lambda}+\bar{\mu}} \frac{l !\left(\lambda_{c}-\bar{\mu}\right) !}{(l-\bar{\mu}) !} \\
& \times\left(\mu_{\mathrm{c}}-n\right) !(Q-n-l) ! \frac{\left(\mu_{\mathrm{c}}-\bar{\mu}\right) !\left(\bar{\lambda}+\mu_{c}+2\right) !}{\left(\mu_{\mathrm{c}}-n-\bar{\mu}\right) !\left(\bar{\lambda}+\mu_{\mathrm{c}}+2-n\right) !}\left[\begin{array}{llll}
\left(\lambda_{\mathrm{c}} 0\right) & (l 0) & (\bar{\lambda} \bar{\mu}) & - \\
\left(0 \mu_{\mathrm{c}}\right) & (Q-l, 0) & (\bar{\lambda} \bar{\mu}) & - \\
\left(\lambda_{\mathrm{c}} \mu_{\mathrm{c}}\right) & (Q 0) & (\lambda \mu) & - \\
- & - & \rho
\end{array}\right]^{2} \\
& \times \frac{[d(\lambda \mu)]^{\frac{1}{2}}}{\lambda_{c} ! \mu_{c} !} \\
& \times\left[\left[P\left(\bar{K}_{1}, \bar{K}_{2}\right)^{\left(\lambda_{c} \mu_{c}\right)} \times P(\bar{K})^{(\mathcal{Q})}\right]^{(\lambda \mu)} \times\left[P\left(\boldsymbol{K}_{1}^{*}, \boldsymbol{K}_{2}^{*}\right)^{\left(\mu_{c} \lambda_{c}\right)} \times P\left(K^{*}\right)^{(0 Q)}\right]^{(\mu \lambda)}\right]_{00}^{(00)} .
\end{aligned}
$$

Eq. (84) gives the internal functions in the form

$$
\left[P\left(\bar{K}_{1}\right)^{\left(\lambda_{c} 0\right)} \times P\left(\bar{K}_{12}\right)^{\left(0 \mu_{c}\right)}\right]_{\alpha_{c}}^{\left(\lambda_{c} \mu_{c}\right)} .
$$

This could also have been transformed to the form

$$
\begin{aligned}
& {\left[P\left(\overline{\boldsymbol{K}}_{1}\right)^{\left(\lambda_{c} 0\right)} \times P\left(\overline{\boldsymbol{K}}_{12}\right)^{\left(0 \mu_{c}\right)}\right]_{\alpha_{c}}^{\left(\lambda_{c} \mu_{c}\right)}} \\
& \quad=\left[\frac{1}{2^{\mu_{c}}}\left(\mu_{c}+1\right) ! \frac{\left(\lambda_{c}+\mu_{c}+1\right)}{\left(\lambda_{c}+1\right)\left(\mu_{c}+1\right)} \frac{\left(\lambda_{c}+\mu_{c}\right) !}{\lambda_{c} ! \mu_{c} !}\right]^{ \pm}\left[P\left(\overline{\boldsymbol{K}}_{1}\right)^{(40)} \times P\left(\overline{\boldsymbol{K}}_{2}\right)^{\left(\mu_{c} 0\right)}\right]_{\alpha_{c}}^{\left(\lambda_{c} \mu_{c}\right)},
\end{aligned}
$$

where, with $\lambda_{\mathrm{c}}+\mu_{c}=4$, eq. (87) has utilized eqs. (60), (B.5), (A.11) with $\lambda_{23}=0$, and (B.1). As in the first variant of our method, eqs. (76)-(80) give the BS transform to within an overall multiplicative constant which must be determined by the proper normalization of the fragment internal functions. Eq. (85) is expressed in terms of properly normalized Bargmann space internal functions, $P\left(\bar{K}_{1}, \bar{K}_{2}\right)^{\left(\lambda_{c} \mu_{c}\right)}$. These are related to the form (86) by

$$
P\left(\bar{K}_{1}, \bar{K}_{2}\right)_{\alpha_{c}}^{\left(\lambda_{c} \mu_{c}\right)}=\left[\lambda_{c} ! \mu_{c} !\right]^{\frac{1}{t}}\left[P\left(\bar{K}_{1}\right)^{\left(\lambda_{c} 0\right)} \times P\left(\bar{K}_{12}\right)^{\left(0 \mu_{c}\right)}\right]_{a}^{\left(\lambda_{c} \mu_{c}\right)} .
$$

The coefficient of the

$$
[d(\lambda \mu)]^{ \pm}\left[\left[P\left(\bar{K}_{1}, \bar{K}_{2}\right)^{\left(\lambda_{c} \mu_{c}\right)} \times P(\bar{K})^{(Q 0)}\right]^{\left(\lambda_{\mu}\right)} \times\left[P\left(K_{1}^{*}, K_{2}^{*}\right)^{\left(\mu_{c} \lambda_{c}\right)} \times P\left(K^{*}\right)^{(0 Q)}\right]^{(\mu \lambda)}\right]_{00}^{(00)}
$$

term gives the overlap of the full SU(3), SU(4) coupled $f+(A-f)$ cluster function 
with itself. This overlap is equal to $1 / N^{2}$, where $N$ is the norm for the SU(3) coupled cluster function $\left[\left(\lambda_{c} \mu_{c}\right) \times(Q 0)\right](\lambda \mu)$ of space symmetry $\left[44 h_{3} h_{4}\right]$. The final result is:

Case 1. For $8 \leqq A-f \leqq 12, f \leqq 4 ; A-f$ fragment of $\left[44 \mu_{c}\right]\left(\lambda_{c} \mu_{c}\right)$ symmetry, $\mu_{\mathrm{c}}=A-f-8$ :

$$
\begin{aligned}
& \frac{1}{\left[N\left(\left(\lambda_{c} \mu_{c}\right)(Q 0)(\lambda \mu)\right)\right]^{2}}=\sum_{l, m, n, p} c_{l, m}\left(\begin{array}{c}
m \\
n
\end{array}\right)\left(\begin{array}{l}
\mathcal{L}-l-m \\
p-l-m
\end{array}\right)(-1)^{n+p} \frac{Q !}{(Q-l-m) !}\left(\frac{A}{f(A-f)}\right)^{l+m} \\
& \times\left[1-p \frac{A}{f(A-f)}\right]^{Q-l-m} \\
& \times \sum_{(\bar{\lambda} \bar{\mu})(\bar{\lambda} \bar{\mu})}(-1)^{\lambda_{c}+l+\bar{\lambda}+\bar{\mu}} \frac{l !\left(\lambda_{c}-\bar{\mu}\right) !\left(\mu_{c}-n\right) !(Q-l-n) !\left(\mu_{c}-\bar{\mu}\right) !\left(\bar{\lambda}+\mu_{c}+2\right) !}{(l-\bar{\mu}) ! \lambda_{c} ! \mu_{c} !(Q-l) !\left(\mu_{c}-n-\bar{\mu}\right) !\left(\bar{\lambda}+\mu_{c}-n+2\right) !} \\
& \times \sum_{\rho}\left[\begin{array}{llll}
\left(\lambda_{c} 0\right) & (l 0) & (\bar{\lambda} \bar{\mu}) & - \\
\left(0 \mu_{c}\right) & (Q-l, 0) & (\bar{\lambda} \bar{\mu}) & - \\
\left(\lambda_{c} \mu_{c}\right) & (Q 0) & (\lambda \mu) & - \\
- & - & \rho
\end{array}\right]^{2},
\end{aligned}
$$

where

for $f \leqq \mu_{c}$,

$[h]=\left[44 \mu_{c} f\right], \quad c_{l, m}=\left(\begin{array}{l}0 \\ i\end{array}\right)\left(\begin{array}{l}f \\ \text { f }\end{array}\right)$,

$[h]=\left[44 \mu_{c}+1 f-1\right], \quad c_{l, m}=\left(\mu_{c}-f+2\right)\left(\begin{array}{l}1 \\ l\end{array}\right)\left(\frac{f-1}{m}\right)-\left(\mu_{c}-f+1\right)\left(\begin{array}{l}0 \\ l\end{array}\right)\left(f_{m}^{f}\right)$,

for $f \geqq \mu_{c}$,

$[h]=\left[44 f \mu_{c}\right], \quad c_{l, m}=\left(\begin{array}{c}f-\mu_{c} \\ t\end{array}\right)\left(\begin{array}{c}\mu_{m} \\ m\end{array}\right)$,

$[h]=[44 f+1 \mu-1], \quad c_{l, m}=\left(f-\mu_{c}+2\right)\left({ }^{f-\mu_{c}+1}\right)\left(\mu_{m}^{\mu_{c}-1}\right)-\left(f-\mu_{c}+1\right)\left({ }^{f-\mu_{c}}\right)\left(\mu_{m}\right)$,

for $f=\mu_{c}=2$,

$[h]=[444], \quad c_{l, m}=6\left(\begin{array}{l}2 \\ l\end{array}\right)\left(\begin{array}{l}0 \\ m\end{array}\right)-6\left(\begin{array}{l}1 \\ l\end{array}\right)\left(\begin{array}{l}1 \\ m\end{array}\right)+1\left({ }_{l}^{0}\right)\left(\begin{array}{l}2 \\ m\end{array}\right)$.

For $f=4$ a similar result, involving $9 U$-coefficients, has been given in ref. ${ }^{26}$ ). Similarly for:

Case $2.12 \leqq A-f \leqq 16, f \leqq 4 ; A-f$ fragment of $\left[4^{3} 4-\mu_{c}\right]$ space symmetry, $\left(\lambda_{c} \mu_{c}\right)=\left(0 \mu_{c}\right)$, with $\mu_{\mathrm{c}}=A-f-12$;

$$
\begin{aligned}
& \frac{1}{\left[N\left(\left(0 \mu_{c}\right)(Q 0)(\lambda \mu)\right)\right]^{2}}=\sum_{l, m, p} c_{l}\left(\begin{array}{l}
f-l \\
m-l
\end{array}\right)\left(\begin{array}{l}
f-m \\
p-m
\end{array}\right)(-1)^{p+l} \frac{(Q-l) !}{(Q-m) !} \\
& \quad \times\left(\frac{A}{f(A-f)}\right)^{m}\left[1-p \frac{A}{f(A-f)}\right]^{Q-m} \frac{\left(\mu_{c}-l\right) !\left(\mu_{c}-\mu\right) !\left(\lambda+\mu_{c}+2\right) !}{\mu_{c} !\left(\mu_{c}-l-\mu\right) !\left(\lambda+\mu_{c}-l+2\right) !},
\end{aligned}
$$


where

for $f \leqq 4-\mu_{c}$;

$[h]=\left[4^{3} 4-\mu_{c} f\right], \quad c_{l}=\left(\begin{array}{l}0 \\ l\end{array}\right)$,

$[h]=\left[4^{3} 4-\mu_{c}+1 f-1\right], c_{l}=\left(4-\mu_{c}-f+2\right)\left(\begin{array}{l}1 \\ l\end{array}\right)-\left(4-\mu_{c}-f+1\right)\left(\begin{array}{l}0 \\ l\end{array}\right)$,

for $f \geqq 4-\mu_{c}$,

$[h]=\left[4^{3} f 4-\mu_{\mathrm{c}}\right], \quad c_{l}=\left({ }^{f+\mu_{c}-4}\right)$,

$\left.\left.[h]=\left[4^{3} f+13-\mu_{c}\right], \quad c_{l}=\left(f-4+\mu_{c}+2\right)()_{i}^{f+\mu_{c}-3}\right)-\left(f-4+\mu_{c}+1\right)()^{f+\mu_{c}-4}\right)$,

for $f=\mu_{c}=2$,

$[h]=\left[4^{4}\right], \quad c_{l}=6\left(\begin{array}{l}2 \\ l\end{array}\right)-6\left(\begin{array}{l}1 \\ l\end{array}\right)+1\left(\left(_{l}^{0}\right)\right.$.

The SU(3) recoupling transformations for this case have been illustrated in some detail in ref. ${ }^{26}$ ) for the special case, $f=4$. The two $U$-coefficients used there, [eq. (31) of ref. $\left.{ }^{26}\right)$ ], have been written explicitly here, through the use of relations (A.13), and (A.14), after use of the symmetry properties (A.8), (A.9), (A.10).

The two cases, $4 \leqq A-f \leqq 8$ and $16 \leqq A-f \leqq 20$, with $\mu_{c}=0$, are very similar.

Case $3 a .4 \leqq A-f \leqq 8, f \leqq 4,\left(\lambda_{c} \mu_{c}\right)=(a 0), a=A-f-4$.

$\frac{1}{[N((a 0)(Q 0)(\lambda \mu))]^{2}}=(-1)^{a+Q+\lambda+\mu} \sum_{m, p} c_{m}\left(\begin{array}{l}f-m \\ p-m\end{array}\right)(-1)^{p} \frac{Q !}{(Q-m) !}$

$$
\times\left(\frac{A}{f(A-f)}\right)^{m}\left[1-p \frac{A}{f(A-f)}\right]^{Q-m}\left[\begin{array}{lll}
(m 0) & (Q-m, 0) & (Q 0) \\
(a-m, 0) & (m 0) & (a 0) \\
(a 0) & (Q 0) & (\lambda \mu)
\end{array}\right]
$$

Case $3 b .16 \leqq A-f \leqq 20, f \leqq 4,\left(\lambda_{c} \mu_{c}\right)=(2 a, 0), a=A-f-16$

$$
\begin{aligned}
& \frac{1}{[N((2 a, 0)(Q 0)(\lambda \mu))]^{2}}=(-1)^{Q+\lambda+\mu} \sum_{l, m, p} c_{m}(f-l)\left(\begin{array}{l}
f-l \\
f-l
\end{array}\right) \frac{(-1)^{p}}{2^{m}} \frac{Q !}{(Q-m-l) !} \\
& \quad \times\left(\frac{A}{f(A-f)}\right)^{m+l}\left[1-p \frac{A}{f(A-f)}\right]^{Q-m-l}\left[\begin{array}{lll}
(2 m 0) & (Q-2 m, 0) & (Q 0) \\
(2 a-2 m, 0) & (2 m 0) & (2 a 0) \\
(2 a 0) & (Q 0) & (\lambda \mu)
\end{array}\right]
\end{aligned}
$$

where, with $b=1$ for case $3 a, b=4$ for case $3 \mathrm{~b}$..

for $f \leqq a$,

$[h]=\left[4^{b} a f\right], \quad c_{m}=\left(\begin{array}{c}f \\ m\end{array}\right)$,

$[h]=\left[4^{b} a+1 f-1\right], \quad c_{m}=(a-f+2)\left(\begin{array}{c}f-1 \\ m\end{array}\right)-(a-f+1)\left(\begin{array}{l}f \\ m\end{array}\right)$,

for $f \geqq a$,

$[h]=\left[4^{b} f a\right], \quad c_{m}=\left(\begin{array}{c}a \\ m\end{array}\right)$,

$\left.[h]=\left[4^{o} f+1 a-1\right], \quad c_{m}=(f-a+2)\left({ }^{a-1}\right)-(f-a+1)_{m}^{a}\right)$,

for $f=a=2$,

$[h]=\left[4^{b+1}\right], \quad c_{m}=6\left(\begin{array}{l}0 \\ m\end{array}\right)-6\left(\begin{array}{l}1 \\ m\end{array}\right)+1\left(\left(_{m}^{2}\right)\right.$. 
The 9- $(\lambda \mu)$ coefficients needed for these cases are equivalent to SU(2) 9-j coefficients, and, with 4 stretched couplings, have the simple form of eq. (A.22). Norms for the special cases $\alpha+{ }^{20} \mathrm{Ne}$ and $\alpha+{ }^{12} \mathrm{C}$ have also been given by Fujiwara and Horiuchi $\left.{ }^{27}\right)$ in very similar form.

As a last example we give the norm for:

Case 4. $20 \leqq A-f \leqq 24, f \leqq 4 ;\left(\lambda_{c} \mu_{c}\right)=\left(8 \mu_{c}\right)$ with $\mu_{c}=A-f-20$.

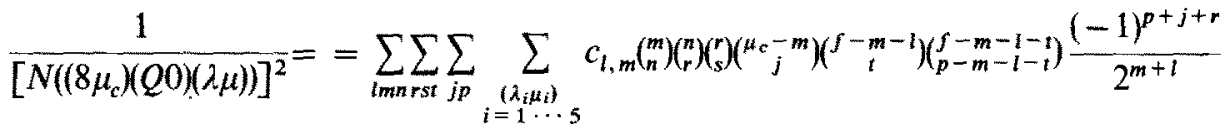

$$
\begin{aligned}
& \times\left(\frac{A}{f(A-f)}\right)^{2 m+2 l+t}\left[1-p \frac{A}{f(A-f)}\right]^{Q-2 m-2 l-t} \\
& \times \frac{Q !}{(Q-2 m-2 l-t) !}(-1)^{Q+\lambda_{1}+\mu_{1}+\lambda_{4}+\mu_{4}+\lambda_{5}+\mu_{5}} \\
& \times\left[\frac{(2 n-2 r+2 s) !(2 n-2 s) !\left(Q-2 n+2 r-2 s-\mu_{4}\right) !\left(Q-2 n+2 s-\mu_{4}\right) !}{(Q-2 n+2 r-2 s) !(Q-2 n+2 s) !}\right]^{\frac{1}{2}} \\
& \times\left[\frac{(2 j+2 r-2 s) !(2 j+2 s) !\left(2 \mu_{c}-2 j-2 s-\mu_{5}\right) !\left(2 \mu_{c}-2 j-2 r+2 s-\mu_{5}\right) !}{\left(2 \mu_{c}-2 j-2 r+2 s\right) !\left(2 \mu_{c}-2 j-2 s\right) !\left(2 n-2 s-\mu_{5}\right) !\left(2 n-2 r+2 s-\mu_{5}\right) !}\right]^{\frac{1}{2}} \\
& \times\left[\begin{array}{c}
(2 m+2 l-2 n+2 r-2 s) !(2 m+2 l-2 n+2 s) !\left(8-2 m-2 l+2 n-2 s-\mu_{1}\right) ! \\
\times\left(8-2 m-2 l+2 n-2 r+2 s-\mu_{1}\right) !
\end{array}\right]^{\frac{1}{2}} \\
& \times f\left(\mu_{c}\right) \sum_{\rho} U^{2}\left(\left(\lambda_{1} \mu_{1}\right)\left(\lambda_{2} \mu_{2}\right)(\lambda \mu)(Q 0) ;\left(8 \mu_{c}\right)_{--} ;\left(\lambda_{3} \mu_{3}\right)_{-} \rho\right) \\
& \times\left[\begin{array}{lll}
(8-2 m-2 l+2 n-2 s, 0) & (2 j+2 s, 0) & \left(\lambda_{1} \mu_{1}\right) \\
(2 m+2 l-2 n+2 s, 0) & \left(2 \mu_{c}-2 j-2 s, 0\right) & \left(\lambda_{2} \mu_{2}\right) \\
(80) & \left(2 \mu_{c}, 0\right) & \left(8 \mu_{c}\right)
\end{array}\right] \\
& \times\left[\begin{array}{lll}
(8-2 m-2 l+2 n+2 s-2 r, 0) & (2 j+2 r-2 s, 0) & \left(\lambda_{1} \mu_{1}\right) \\
(2 m+2 l-2 n-2 s+2 r, 0) & \left(2 \mu_{c}-2 j-2 r+2 s, 0\right) & \left(\lambda_{2} \mu_{2}\right) \\
(80) & \left(2 \mu_{c}, 0\right) & \left(8 \mu_{c}\right)
\end{array}\right] \\
& \times \sum_{\rho^{\prime}}\left[\begin{array}{llll}
(2 m+2 l-2 n+2 s, 0) & (Q-2 n+2 r-2 s, 0) & \left(\lambda_{4} \mu_{4}\right) & - \\
\left(2 \mu_{c}-2 j-2 s, 0\right) & (2 n-2 r+2 s, 0) & \left(\lambda_{5} \mu_{5}\right) & - \\
\left(\lambda_{2} \mu_{2}\right) & (Q 0) & \left(\lambda_{3} \mu_{3}\right) & - \\
- & - & \rho^{\prime}
\end{array}\right]
\end{aligned}
$$




$$
\times\left[\begin{array}{llll}
(2 m+2 l-2 n+2 r-2 s, 0) & (Q-2 n+2 s, 0) & \left(\lambda_{4} \mu_{4}\right) & - \\
\left(2 \mu_{c}-2 j-2 r+2 s, 0\right) & (2 n-2 s, 0) & \left(\lambda_{5} \mu_{5}\right) & - \\
\left(\lambda_{2} \mu_{2}\right) & (Q 0) & \left(\lambda_{3} \mu_{3}\right) & - \\
- & - & \rho^{\prime} &
\end{array}\right] .
$$

The $c_{l, m}$ for $\left[4^{5} h^{\prime} h^{\prime \prime}\right]$ are identical with the $c_{l, m}$ for $\left[4^{2} h^{\prime} h^{\prime \prime}\right]$ of case 1 , see eq. (89b).

TABLE 5

The factors $f\left(\mu_{c}\right)$

\begin{tabular}{cc}
\hline$\mu_{c}$ & $f\left(\mu_{c}\right)$ \\
\hline 0 & 1 \\
1 & $\frac{4}{5}$ \\
2 & $\frac{42}{55}$ \\
3 & $\frac{7}{11}$ \\
4 & $\frac{5 \cdot 7^{2}}{4 \cdot 11 \cdot 13}$ \\
\hline
\end{tabular}

The $f\left(\mu_{c}\right)$ are given in table 5. They are related to the normalization of the heavyfragment internal wave functions,

$$
\left[P\left(\bar{K}_{4}\right)^{(80)} \times P\left(\bar{K}_{5}\right)^{\left(2 \mu_{c} 0\right)}\right]_{\alpha_{c}}^{\left(8 \mu_{c}\right)},
$$

and are related to $4+\left(n=\mu_{c}\right) \rightarrow\left(4+\mu_{c}\right)$ particle sd shell fractional parentage coefficients. [The inverse of these numbers can be found in tables A.1 through A.4 of ref. $\left.{ }^{54}\right)$.] The first two of the four $9-(\lambda \mu)$ coefficients in eq. (94) are equivalent to simple $9-j$ coefficients, eq. (A.21).

In the more challenging of these five cases a few bona fide SU(3) 6-( $(\lambda \mu)$ and 9-( $(\lambda \mu)$ coefficients are needed for the evaluation of the norms. These are readily available through the codes of Akiyama and Draayer ${ }^{36}$ ).

Norm and overlap matrix elements are particularly simple in all those cases in which one of the fragments has an internal function of SU(3) symmetry (00), the second an internal $\left(\lambda_{c} \mu_{c}\right)$ with either $\lambda_{c}=0$, or $\mu_{c}=0$. All such matrix elements can be evaluated without the use of bona fide SU(3) recoupling coefficients.

There are other examples of this kind. If one of the fragments is a heavier closed shell nucleus, such as ${ }^{16} \mathrm{O}$ or ${ }^{40} \mathrm{Ca}$, the second fragment a nucleus with an internal function with either $\lambda_{c}=0$, or $\mu_{c}=0$, the SU(3) structure of norm and overlap matrix elements is identical with that of cases 2 and 3 above. Specific examples of this kind, 
needed for the ${ }^{12} \mathrm{C}+{ }^{12} \mathrm{C}$ resonance problem, (sect. 6), are the norm of the ${ }^{8} \mathrm{Be}+{ }^{16} \mathrm{O}$ cluster functions, and the overlap between ${ }^{8} \mathrm{Be}+{ }^{16} \mathrm{O}$ and $\alpha+{ }^{20} \mathrm{Ne}$ cluster functions. These can also be evaluated from single-column functions tabulated in appendix $\mathrm{C}$ and lead to expressions very similar to case 3 . In particular:

(a) Norm of ${ }^{8} \mathrm{Be}+{ }^{16} \mathrm{O}$ cluster functions. With a ${ }^{8} \mathrm{Be}$ internal function of $\mathrm{SU}(3)$ symmetry $\left(\lambda_{c} \mu_{c}\right)=(40)$

$$
\begin{aligned}
\frac{1}{[N((40)(Q 0)(\lambda \mu))]^{2}} & =\left\langle\phi ( { } ^ { 1 6 } \mathrm { O } ) ^ { ( 0 0 ) } [ \phi ( { } ^ { 8 } \mathrm { Be } ) ^ { ( 4 0 ) } \times \chi ( R ) ^ { ( Q 0 ) } ] _ { \alpha } ^ { ( \lambda \mu ) } | \mathscr { A } | \phi ( { } ^ { 1 6 } \mathrm { O } ) ^ { ( 0 0 ) } \left[\phi\left({ }^{8} \mathrm{Be}\right)^{(40)}\right.\right. \\
& \left.\left.\left.\times \chi(R)^{(Q 0)}\right]_{\alpha}^{(\lambda \mu)}\right\rangle=(-1)^{Q+\lambda+\mu} \sum_{l m n} \sum_{p}\left({ }_{l}^{4}\right)\left(l_{n}\right)\left({ }_{m}^{n}-l\right)()_{p-l}^{8-m}\right)(-1)^{l+m+n+p} \\
& \times \frac{Q !}{(Q-m) !}\left(\frac{3}{16}\right)^{m}\left[1-p \frac{3}{16}\right]^{Q-m}\left[\begin{array}{lll}
(n 0) & (Q-n, 0) & (Q 0) \\
(4-n, 0) & (n 0) & (40) \\
(40) & (Q 0) & (\lambda \mu)
\end{array}\right]
\end{aligned}
$$

(b) Overlap between ${ }^{8} \mathrm{Be}+{ }^{16} \mathrm{O}$ and $\alpha+{ }^{20} \mathrm{Ne}$ cluster functions. The overlap matrix elements are given by

$$
\begin{aligned}
\left\langle\phi\left({ }^{16} \mathrm{O}\right)^{(00)}\right. & {\left.\left[\phi\left({ }^{8} \mathrm{Be}\right)^{(40)} \times \chi(\bar{R})^{(Q+4,0)}\right]_{\alpha}^{(\lambda \mu)}|\mathscr{A}| \phi(\alpha)^{(00)}\left[\phi\left({ }^{20} \mathrm{Ne}\right)^{(80)} \times \chi(R)^{(Q 0)}\right]_{\alpha}^{(\lambda \mu)}\right\rangle } \\
& \left.\left.=\sum_{l m p}\left({ }_{l}^{4}\right) \mathcal{l}_{m}^{l}\right)()_{p-m}^{4-m}\right) \frac{(-1)^{l+m+p}}{2^{l-m-2}} \frac{[Q !(Q+4) !]^{\frac{1}{2}}}{(Q-l) !} \\
& \times\left(\frac{3}{16}\right)^{2}\left(\frac{3}{4 \sqrt{10}}\right)^{l}\left[\frac{8-3 p}{4 \sqrt{10}}\right]^{Q-l}\left[\begin{array}{lll}
(Q+m-l, 0) & (l-m, 0) & (Q 0) \\
(4+l-m, 0) & (4-l+m, 0) & (80) \\
(Q+4,0) & (40) & (\lambda \mu)
\end{array}\right]
\end{aligned}
$$

\section{Spectroscopic amplitudes for the ${ }^{12} \mathrm{C}+{ }^{12} \mathrm{C}$ resonances}

The ${ }^{12} \mathrm{C}+{ }^{12} \mathrm{C}$ excitation functions are determined by the partial width amplitudes, $\Gamma_{v c}^{\frac{1}{2}}$ and $\Gamma_{v c^{\prime}}^{\frac{1}{2}}$. For given entrance and exit channels, $c$ and $c^{\prime}$, and quasibound states $v$ of very similar structure, the relative magnitudes of $\left(\Gamma_{v c}^{\frac{1}{2}} \Gamma_{v c^{\prime}}^{\frac{1}{2}}\right)$ for different $v$ can be expected to be proportional to the relative magnitudes of the product of amplitudes, $\left(A_{v e} A_{v c^{\prime}}\right)$. The spectroscopic amplitudes $A_{v c^{\prime}}=\left\langle v \mid c^{\prime}\right\rangle$ can therefore give some measure of the relative strengths of the excitation functions observed in specific channels $c^{\prime}$. The quasi-bound states $|v\rangle$ are to be built from a restricted basis $|i\rangle$. The states $|i\rangle$ are the Pauli-allowed eigenvectors of the ${ }^{12} \mathrm{C}+{ }^{12} \mathrm{C}$ "molecular states"

$$
\mathscr{A}\left|\left[\left[\phi\left({ }^{12} \mathrm{C}\right)^{(04)} \times \phi\left({ }^{12} \mathrm{C}\right)^{(04)}\right]^{\left(\lambda_{c} \mu_{c}\right)} \times \chi(R)^{(14,0)}\right]_{\kappa J M_{J}}^{\left(\lambda_{\mu}\right)}\right\rangle \equiv \mathscr{A}\left|\left(\lambda_{c} \mu_{c}\right)(\lambda \mu)\right\rangle,
$$

with $\kappa=\mu, \mu-2, \ldots, 0$ or 1 . These states, with $Q=14$, are the shell-model components of $2 \hbar \omega$ oscillator excitation of the ${ }^{12} \mathrm{C}+{ }^{12} \mathrm{C}$ cluster functions. The states $|i\rangle$ are the eigenvectors of the operator $1 \mathscr{A}$, (with eigenvalues different from zero), in this re- 
stricted $\left|\left(\lambda_{c} \mu_{c}\right)(\lambda \mu)\right\rangle$ basis. States $|j\rangle$ with eigenvalue zero are Pauli forbidden. They are the states which are annihilated by the operator $\mathscr{A}$. The states $|i\rangle$, with

$$
\mathscr{A}|i\rangle=\Lambda_{i}|i\rangle \quad\left(\Lambda_{i} \neq 0\right)
$$

and

$$
|i\rangle=\sum_{\left(\lambda_{c} \mu_{c}\right)}\left|\left(\lambda_{c} \mu_{c}\right)(\lambda \mu)\right\rangle\left\langle\left(\lambda_{c} \mu_{c}\right)(\lambda \mu) \mid i\right\rangle
$$

require quantum numbers $(\lambda \mu) \kappa J M_{J}$, as well as an index $i$, for their full specification. The transformation coefficients $\left\langle\left(\lambda_{c} \mu_{c}\right)(\lambda \mu) \mid i\right\rangle$ are independent of $\kappa J M_{J}$, and, for fixed $Q$ and fixed $\left(\lambda_{f} \mu_{f}\right),\left(\lambda_{A-s} \mu_{A-s}\right)$, are specified by the quantum numbers $\left(\lambda_{c} \mu_{c}\right)$, $(\lambda \mu)$. It will be assumed that the quasi-bound states have the form $|v\rangle=\sum_{i}|i\rangle\langle i \mid v\rangle$. Before attempting to solve the quasi-bound state eigenvalue problem, it will be interesting to calculate the amplitudes $A_{i c}$, (rather than $A_{v c}$ ), since the relative magnitudes $A_{i c^{\prime}}$ for different channels $c^{\prime}$ may give an indication of the importance of a component $|i\rangle$ in a particular resonance fine structure peak. The spectroscopic amplitudes $A_{\mathfrak{i c}^{\prime}}$ are defined by

$$
A_{i c^{\prime}}=\frac{1}{A_{i}^{\frac{2}{2}}}\left\langle i|\mathscr{A}| c^{\prime}\right\rangle
$$

that is, they give the projection of the normalized, fully antisymmetrized state $|i\rangle$ on the state $\left|c^{\prime}\right\rangle$.

In this section we calculate the norm and overlap matrix elements needed for the ${ }^{12} \mathrm{C}+{ }^{12} \mathrm{C}$ norm eigenvalue problem, and for the evaluation of the spectroscopic amplitudes $A_{i c^{\prime}}$ for the channels $c^{\prime}$ enumerated in sect. 2 .

The ${ }^{12} \mathrm{C}+{ }^{12} \mathrm{C}$ overlaps. Matrix elements between ${ }^{12} \mathrm{C}+{ }^{12} \mathrm{C}$ cluster functions of the type

$$
\begin{aligned}
\left\langle\left[\left[\phi\left({ }^{12} \mathrm{C}\right)^{(04)} \times \phi\left({ }^{12} \mathrm{C}\right)^{(04)}\right]^{\left(\lambda_{c} \mu_{c}\right)} \times \chi(R)^{(Q 0)}\right]_{\alpha}^{(\lambda \mu)}\right| \mathscr{A} \\
\times\left|\left[\left[\phi\left({ }^{(2} \mathrm{C}\right)^{(04)} \times \phi\left({ }^{12} \mathrm{C}\right)^{(04)}\right]^{\left(\lambda_{c}^{\prime} \mu_{c}^{\prime}\right)} \times \chi(R)^{(Q 0)}\right]_{\alpha}^{(\lambda \mu)}\right\rangle
\end{aligned}
$$

are calculated most efficiently by the second variant of our method, the "singlecolumn technique". A number of checks have also been made with the full "4-columned method" especially for those $\left(\lambda_{c} \mu_{c}\right),\left(\lambda_{c}^{\prime} \mu_{\mathrm{c}}^{\prime}\right)$ combinations for which the total number of $y_{m\left(\lambda^{\prime} \mu^{\prime}\right)}$ and $D_{m}(p)$ is not too large. For states of positive parity, (even $Q$-values), the only possible $\left(\lambda_{c} \mu_{c}\right)$ values are $\left(\lambda_{c} \mu_{c}\right)=(08),(24)$ and $(40)$, so that the norm eigenmatrix may be at most a $3 \times 3$ matrix for some $(\lambda \mu)$ 's. For the states with $Q=14$, discussed in connection with table 1 , only $(\lambda \mu)=(10,4)$ leads to a $3 \times 3$ matrix. States with Pauli-forbidden $(\lambda \mu)$ values must have zero matrix elements. This so-called redundant solution test furnishes a check on all calculations. Even more powerful test cases exist, however. For some $(\lambda \mu) Q$ combinations only a single Pauli-allowed state may exist, even though the cluster basis has a dimension greater than 1 . The special cases $(\lambda \mu)=(14,2)$ and $(11,5)$ for $Q=14$ are examples. In such cases the 
norm matrix can have only one non-zero eigenvalue, corresponding to the Pauliallowed eigenvector. The remaining eigenvalues must be zero, even if the number of channels is increased by including other cluster structures in the basis.

The single-column BS transforms needed for the ${ }^{12} \mathrm{C}+{ }^{12} \mathrm{C}$ basis can be found in appendix C. They are the BS transforms of the operator 1 12 for 6-particle systems with cluster decomposition into fragments with $l+n=l^{\prime}+n^{\prime}=3+3$ particles. The 3 nucleons in each fragment must couple to $\mathrm{SU}(3)$ symmetry $\left(\lambda_{l} \mu_{l}\right)=\left(\lambda_{n} \mu_{n}\right)=(01)$. The resultant single-column $\left(\lambda_{c} \mu_{c}\right)$ has the two possible values $\left(\lambda_{c} \mu_{c}\right)=(02)$ and (10). The single-column BS transforms can be expressed in terms of the relative motion degree of freedom, $K$, and the two pseudovectors $K_{12}=\sqrt{\frac{1}{2}}\left[K_{1} \times K_{2}\right], K_{34}=$ $\sqrt{\frac{1}{2}}\left[K_{3} \times K_{4}\right]$, which carry the internal excitations of the two ${ }^{12} \mathrm{C}$ fragments, see fig. 1b. The needed single-column BS transforms are given in terms of the $K$-space functions $H\left({ }_{3}^{3\left(\lambda_{c} \mu_{c}\right) 3\left(\lambda_{c}^{\prime} \mu_{c}^{\prime}\right)}\right)$ in appendix C. Note that the cross term, with $\left(\lambda_{c} \mu_{c}\right)=(02)$, $\left(\lambda_{c}^{\prime} \mu_{c}^{\prime}\right)=(10)$, is zero because of the identity of the two fragments. The BS transform for the full 24-particle system is built from the symmetrized combination of these four single-column functions

$$
H\left(\bar{K}, K^{*}\right)=\frac{1}{2}\left(\frac{1}{4 !}\right)^{2} \mathrm{e}^{\left(\mathbf{K} \cdot \mathbf{K}^{*}\right)} \sum_{a}\left(\begin{array}{l}
4 \\
a
\end{array}\right)\left[H\left({ }_{3}^{3(02) 3(02)}\right)\right]^{4-a}\left[H\left(\begin{array}{l}
3(10) 3(10) \\
3
\end{array}\right]^{a} .\right.
$$

The space symmetry is uniquely determined, since four single-column functions of $l+n=3+3$ character can combine only to space symmetry [ $\left.4^{6}\right]$. The final $\left(\lambda_{c} \mu_{c}\right)$ are built from $(4-a)$ symmetrically coupled (02) representations coupled with $a$ symmetrically coupled (10) representations. Note that terms with odd- $a$ decouple from those with even- $a$. Terms with $a=$ odd can only lead to $\left(\lambda_{c} \mu_{c}\right)$ eigenvalues of (16) and (32):

$$
\begin{aligned}
(02)_{\text {symm }}^{3} \times(10) \rightarrow\{(06)+(22)+(00)\} \times(10) \rightarrow & (16) \text { and (32) only } \\
& (02) \times(10)_{\text {symm }}^{3} \rightarrow(02) \times(30) \rightarrow(32) \text { only. }
\end{aligned}
$$

These lead to negative-parity states only. Terms with odd $a$ are thus split off from the terms with even $a$ in eq. (100). For positive-parity states

$$
\begin{aligned}
& H\left(\bar{K}, K^{*}\right)_{+}=\frac{1}{2}\left(\begin{array}{r}
1 \\
4 !
\end{array}\right)^{2} \mathrm{e}^{\left(\overline{\mathbf{K}} \cdot \mathbf{K}^{*}\right)}\left\{\left[H\left({ }_{3}^{3(02) 3(02)}\right)\right]^{4}\right. \\
&\left.+6\left[H\left({ }_{3}^{3(02) 3(02)}\right)\right]^{2}\left[H\left({ }_{3}^{3(10) 3(10)}\right)\right]^{2}+\left[H\left({ }_{3}^{3(10) 3(10)}\right)\right]^{4}\right\} .
\end{aligned}
$$

The factors $\frac{1}{2}$, (related to the identity of the two fragments), and $(4 !)^{-2}$ are required to gain the proper normalization of the fragment internal wave functions. In terms of the shorthand notation

$$
\begin{aligned}
& \mathscr{P}^{\left(\lambda_{c} \mu_{c}\right)\left(\lambda_{c}^{\prime} \mu_{c}^{\prime}\right)} \equiv[d(\lambda \mu)]^{\frac{1}{1}}[[\left.P\left(\bar{K}_{12}, \bar{K}_{34}\right)^{\left(\lambda_{c} \mu_{c}\right)} \times P(\bar{K})^{(\boldsymbol{Q} 0)}\right]^{(\lambda \mu)} \\
&\left.\times\left[P\left(K_{12}^{*}, K_{34}^{*}\right)^{\left(\mu_{c}^{\prime} \lambda_{c}^{\prime}\right)} \times P\left(K^{*}\right)^{(0 Q)}\right]^{(\mu \lambda)}\right]_{00}^{(00)}
\end{aligned}
$$


for the basic $K$-space expansion functions, the last term of eq. (101) can give rise only to terms $\mathscr{P}^{(40)(40)}$. Using straightforward $S U(3)$ recoupling transformations of the type illustrated by the examples of sect. 5 , this term can be expanded by

$$
\begin{aligned}
\frac{1}{2}\left(\frac{1}{4 !}\right)^{2} \mathrm{e}^{\left(\overline{\mathbf{K}} \cdot \mathbf{K}^{*}\right)}\left[H\left({ }_{3}^{3(10) 3(10)}\right)\right]^{4}=5 \sum_{Q(\lambda \mu)}(-1)^{Q+\lambda+\mu}\left[\frac{d(Q 0)}{d(\lambda \mu)}\right]_{m n l}^{\frac{1}{2}} \sum_{j p} \frac{(-1)^{m+n+j}}{2^{5+m-2 l}} \\
\left.\left.\left.\times\left({ }_{l}^{4}\right)\left(\begin{array}{l}
l \\
n
\end{array}\right)\left(m_{m-l}^{n}\right)\right)_{j}^{8-m}\right)_{p-l-j}^{4+m-2 l}\right)\left(\frac{A}{f(A-f)}\right)^{m}\left[1-p \frac{A}{f(A-f)}\right]^{Q-m} \frac{Q !}{(Q-m) !} \\
\times \sum_{(\overline{\bar{\lambda}} \bar{\mu})}\left[\frac{d(\bar{\lambda} \bar{\mu})}{d(n 0)}\right]^{\frac{1}{2}} \frac{n !(4-\bar{\mu}) !}{(n-\overline{\bar{\mu}}) ! 4 !} U((04)(0 Q)(\bar{\mu} \bar{\lambda})(Q-n, 0) ;(\mu \lambda)(0 n)) \\
\times U((40)(n 0)(\lambda \mu)(Q-n, 0) ;(\bar{\lambda} \bar{\mu})(Q 0)) \mathscr{P}^{(40)(40)} .
\end{aligned}
$$

With $(\lambda \mu)=(Q+4-2 \mu, \mu), \mu=0, \ldots, 4$; the two $U$-coefficients of this term are equivalent to simple SU(2) $U$-coefficients, eq. (A.11). (Note that $f=A-f=12$.) The first term of eq. (101a), with $a=0$, gives

$$
\begin{aligned}
& \frac{\mathrm{e}^{\left(\overline{\mathbf{K}} \cdot \mathbf{K}^{*}\right)}}{2(4 !)^{2}}\left[H\left(\begin{array}{l}
3(02) 3(02) \\
3
\end{array}\right]^{4}=\sum_{\left(\lambda_{c} \mu_{c}\right)\left(\lambda_{c}^{\prime} \mu^{\prime}\right)=(08)(24)(40)} \sum_{Q(\lambda \mu)}(-1)^{Q+\lambda+\mu}\right. \\
& \times\left[\frac{d(Q 0) d\left(\lambda_{c} \mu_{c}\right) d\left(\lambda_{c}^{\prime} \mu_{c}^{\prime}\right)}{d(\lambda \mu)}\right]^{\frac{1}{2}} \\
& \left.\left.\times \sum_{l j m n} \sum_{r s t} \sum_{p} \frac{(-1)^{p}}{2^{5+m-j}}\left({ }_{l}^{4}\right) X_{j}^{l}\right)\left(_{n}^{4-j}\right)\left({ }_{m}^{j}\right) X_{r}^{m}\right)\left({ }_{s}^{r}\right)\left({ }_{t}^{s}\right)\left({ }_{p-l}^{12-2 l}\right) \\
& \times \frac{Q !}{(Q-2 l) !}\left(\frac{A}{f(A-f)}\right)^{2 l}\left[1-p \frac{A}{f(A-f)}\right]^{Q-2 l} \sum_{(\bar{\lambda} \bar{\mu})} \frac{(-1)^{\bar{\lambda}+\bar{\mu}}}{[d(\bar{\lambda} \bar{\mu}) d(2 j-m, 0)]^{\frac{1}{2}}} \\
& \times\left[\begin{array}{lll}
(4-j+m-r+s-t, 0) & (j-m+r-s+t, 0) & (40) \\
(4-j+r-s+t, 0) & (j-r+s-t, 0) & (40) \\
(\bar{\lambda} \bar{\mu}) & (2 j-m, 0) & \left(\mu_{c} \lambda_{c}\right)
\end{array}\right] \\
& \times\left[\begin{array}{lll}
(4-j+m-r+t, 0) & (4-j+r-t, 0) & (\bar{\lambda} \bar{\mu}) \\
(j-m+r-t, 0) & (j-r+t, 0) & (2 j-m, 0) \\
(40) & (40) & \left(\mu_{c}^{\prime} \lambda_{c}^{\prime}\right)
\end{array}\right] \\
& \times\left[\begin{array}{lll}
(4-j+m-n-r, 0) & (n+s-t, 0) & (4-j+m-r+s-t, 0) \\
(n+t, 0) & (4-j-n+r-s, 0) & (4-j+r-s+t, 0) \\
(4-j+m-r+t, 0) & (4-j+r-t, 0) & (\bar{\lambda} \bar{\mu})
\end{array}\right] \\
& \times U\left(\left(\mu_{c}^{\prime} \lambda_{c}^{\prime}\right)(0 Q)(\bar{\lambda} \bar{\mu})(Q-2 j+m, 0) ;(\mu \lambda)(0,2 j-m)\right) \\
& \times U\left(\left(\lambda_{c} \mu_{c}\right)(2 j-m, 0)(\lambda \mu)(Q-2 j+m, 0) ;(\bar{\mu} \bar{\lambda})(Q 0)\right) \times \mathscr{P}^{\left(\lambda_{c} \mu_{c}\right)\left(\lambda_{c}^{\prime} \mu_{c}^{\prime}\right)} .
\end{aligned}
$$


The three 9-( $\lambda \mu)$ coefficients are all equivalent to $S U(2) 9 j$ coefficients. This term con tributes to all three $\left(\lambda_{c} \mu_{c}\right)$ and $\left(\lambda_{c}^{\prime} \mu_{c}^{\prime}\right)$ values.

Finally, the middle term of eq. (101), with $a=2$, contributes to terms with $\left(\lambda_{c} \mu_{c}\right.$ and $\left(\lambda_{c}^{\prime} \mu_{c}^{\prime}\right)=(24)$ and $(40)$ only:

$$
\begin{aligned}
& \frac{6}{2(4 !)^{2}} \mathrm{e}^{\left(\overline{\mathbf{K}} \cdot \mathbf{K}^{*}\right)}\left[H\left({ }_{3}^{3(02) 3(02)}\right)\right]^{2}\left[H\left({ }_{3}^{3(10) 3(10)}\right)\right]^{2} \\
& =\sum_{\left(\lambda_{c} \mu_{c}\right)\left(\lambda_{c}^{\prime} \mu_{c}^{\prime}\right)=(24)(40)} \sum_{Q(\lambda \mu)}(-1)^{Q+\lambda+\mu_{3}}\left[\frac{d(Q 0) d\left(\bar{\lambda}_{c} \bar{\mu}_{c}\right) d\left(\bar{\lambda}_{c}^{\prime} \bar{\mu}_{c}^{\prime}\right)}{d(\lambda \mu)}\right]^{\frac{1}{2}} \\
& \times \sum_{l j m n} \sum_{r s t} \sum_{p q} \sum_{\alpha \beta \gamma} \frac{(-1)^{l+q+\beta+\gamma}}{2^{5+\beta-2 \alpha-j+m}}\left(\begin{array}{l}
2 \\
l
\end{array}\right)\left(\begin{array}{l}
l \\
j
\end{array}\right)\left(\begin{array}{l}
2-j \\
n
\end{array}\right)\left({ }_{m}^{j}\right)\left(\begin{array}{l}
m \\
r
\end{array}\right)\left({ }_{s}\right)\left(\begin{array}{l}
(j) \\
t
\end{array}\right) \\
& \times\left({ }_{\alpha}^{2}\right)\left({ }_{\gamma}^{\alpha}\right)\left({ }_{\beta}^{\beta}-\alpha\right)\left({ }^{10-2 l-\beta}\right)\left(\begin{array}{c}
2+\beta-2 \alpha \\
p-\alpha-l-q
\end{array}\right) \frac{Q !}{(Q-2 l-\beta) !}\left(\frac{A}{f(A-f)}\right)^{2 l+\beta}\left[1-p \frac{A}{f(A-f)}\right]^{Q-2 l-\beta} \\
& \times \sum_{(\overline{\bar{\lambda}} \bar{\mu})(\overline{\bar{\lambda}} \overline{\bar{\mu}})} \sum_{\left(\lambda^{\prime \prime} \mu^{\prime \prime}\right)} \frac{(-1)^{\lambda^{\prime \prime}+\mu^{\prime \prime}+\overline{\bar{\lambda}}+\bar{\mu}}}{d(\bar{\lambda} \bar{\mu})}\left[\frac{d\left(\lambda^{\prime \prime} \mu^{\prime \prime}\right)}{d(2 j-m+\gamma, 0)}\right]^{\frac{t}{2}} \frac{(2-\overline{\bar{\mu}}) ! \gamma !}{2 !(\gamma-\overline{\bar{\mu}}) !} \\
& \times\left[\frac{1}{6} \sqrt{7} \delta_{\left(\lambda_{c} \mu_{c}\right)(24)}+\frac{1}{3} \sqrt{5} \delta_{\left(\lambda_{\varepsilon} \mu_{c}\right)(40)}\right]\left[\frac{1}{6} \sqrt{7} \delta_{\left(\lambda_{c}^{\prime} \mu_{c}^{\prime}\right)(24)}+\frac{1}{3} \sqrt{5} \delta_{\left(\lambda_{c}^{\prime} \mu_{c}^{\prime}\right)(40)}\right] \\
& \times\left[\begin{array}{lll}
(2-j+m-r+s-t, 0) & (j-m+r-s+t, 0) & (20) \\
(2-j+r-s+t, 0) & (j-r+s-t, 0) & (20) \\
(\bar{\lambda} \bar{\mu}) & (2 j-m, 0) & \left(\bar{\mu}_{c} \bar{\lambda}_{c}\right)
\end{array}\right] \\
& \times\left[\begin{array}{lll}
(2-j+m-r+t, 0) & (2-j+r-t, 0) & (\bar{\lambda} \bar{\mu}) \\
(j-m+r-t, 0) & (j-r+t, 0) & (2 j-m, 0) \\
(20) & (20) & \left(\bar{\mu}_{c}^{\prime} \bar{\lambda}_{c}^{\prime}\right)
\end{array}\right] \\
& \times\left[\begin{array}{lll}
(2-j+m-n-r, 0) & (n+s-t, 0) & (2-j+m-r+s-t, 0) \\
(n+t, 0) & (2-j-n+r-s, 0) & (2-j+r-s+t, 0) \\
(2-j+m-r+t, 0) & (2-j+r-t, 0) & (\bar{\lambda} \bar{\mu})
\end{array}\right] \\
& \times\left[\begin{array}{lll}
\left(\bar{\lambda}_{c} \bar{\mu}_{c}\right) & (2 j-m, 0) & (\bar{\mu} \bar{\lambda}) \\
(20) & (\gamma 0) & (\bar{\lambda} \bar{\mu}) \\
\left(\lambda_{c} \mu_{c}\right) & (2 j-m+\gamma, 0) & \left(\lambda^{\prime \prime} \mu^{\prime \prime}\right)
\end{array}\right]\left[\begin{array}{llc}
\left(\bar{\lambda}_{c}^{\prime} \bar{\mu}_{c}^{\prime}\right) & (2 j-m, 0) & (\bar{\mu} \bar{\lambda}) \\
(20) & (\gamma 0) & (\bar{\lambda} \bar{\mu}) \\
\left(\lambda_{c}^{\prime} \mu_{c}^{\prime}\right) & (2 j-m+\gamma, 0) \cdot\left(\lambda^{\prime \prime} \mu^{\prime \prime}\right)
\end{array}\right] \text {. } \\
& \times U\left(\left(\mu_{c}^{\prime} \lambda_{c}^{\prime}\right)(0 Q)\left(\mu^{\prime \prime} \lambda^{\prime \prime}\right)(Q-2 j+m-\gamma, 0) ;(\mu \lambda)(0,2 j-m+\gamma)\right) \\
& \times U\left(\left(\lambda_{c} \mu_{c}\right)(2 j-m+\gamma, 0)(\lambda \mu)(Q-2 j+m-\gamma, 0) ;\left(\lambda^{\prime \prime} \mu^{\prime \prime}\right)(Q 0)\right) \mathscr{P P}^{\left(\lambda_{c} \mu_{c}\right)\left(\lambda_{c}^{\prime} \mu_{c}^{\prime}\right),}
\end{aligned}
$$

where

$$
\left(\bar{\lambda}_{c} \bar{\mu}_{c}\right)= \begin{cases}(04), & \text { for }\left(\lambda_{c} \mu_{c}\right)=(24) \\ (20), & \text { for }\left(\lambda_{c} \mu_{c}\right)=(40)\end{cases}
$$

Similarly, for $\left(\bar{\lambda}_{c}^{\prime} \bar{\mu}_{c}^{\prime}\right)$ and $\left(\lambda_{c}^{\prime} \mu_{c}^{\prime}\right)$ 
The combination of eqs. (101)-(104), together with the basic relation (29), now leads to the norm matrix elements of eq. (99). The states with $Q=14=[Q$ (minimum Pauli allowed) +2$]$ form the basis set for our model of molecular quasi-bound states. The eigenvectors $|(\lambda \mu) i\rangle$ and eigenvalues $\Lambda_{i}$ for this value of $Q$ are shown in table 6 .

TABLE 6

${ }^{12} \mathrm{C}+{ }^{12} \mathrm{C}$ norm eigenvalues and vectors for $Q=14$

\begin{tabular}{|c|c|c|c|c|c|}
\hline \multirow{2}{*}{\multicolumn{2}{|c|}{$(\lambda \mu) i$}} & \multirow{2}{*}{$\Lambda_{i}$} & \multicolumn{3}{|c|}{$\left\langle\left[\left(\lambda_{c} \mu_{c}\right) \times(14,0)\right](\lambda \mu) \mid(\lambda \mu) i\right\rangle$} \\
\hline & & & $\left(\lambda_{c} \mu_{c}\right)=(08)$ & $\left(\lambda_{c} \mu_{c}\right)=(24)$ & $\left(\lambda_{c} \mu_{c}\right)=(40)$ \\
\hline$(14,2)$ & 1 & 0.009763 & & 0.95806 & 0.28656 \\
\hline$(11,5)$ & 1 & 0.100713 & 0.98029 & 0.19758 & \\
\hline$(12,3)$ & 1 & 0.040764 & & 0.92236 & 0.38633 \\
\hline$(13,1)$ & 1 & 0.032765 & & 1 & \\
\hline$(10,4)$ & 1 & 0.317811 & 0.91645 & 0.37586 & 0.13732 \\
\hline$(10,4)$ & 2 & 0.086273 & -0.39731 & 0.81384 & 0.42404 \\
\hline$(11,2)$ & 1 & 0.058844 & & 1 & \\
\hline$(12,0)$ & 1 & 0.050513 & & 1 & \\
\hline (93) & 1 & 0.318246 & 0.97842 & 0.20661 & \\
\hline (93) & 2 & 0.070937 & -0.20661 & 0.97842 & \\
\hline$(10,1)$ & 1 & 0.057773 & & 1 & \\
\hline (82) & 1 & 0.304046 & 0.99549 & 0.09492 & \\
\hline (82) & 2 & 0.059327 & -0.09492 & 0.99549 & \\
\hline (71) & 1 & 0.277100 & 1 & & \\
\hline$(60)$ & 1 & 0.252095 & 1 & & \\
\hline
\end{tabular}

Pauli-forbidden states with $\Lambda_{i}=0$ are not included.

Eigenvectors for Pauli-forbidden states can be constructed from the above, using their orthogonality with Pauli-allowed eigenvectors.

The quantum numbers $\kappa J M_{J}$ are omitted since the transformation coefficients are independent of these subgroup labels. The normalized, fully antisymmetrized state vectors are given by

$$
\frac{1}{A_{i}^{\frac{1}{2}}} \mathscr{A}\left|(\lambda \mu) i \kappa J M_{J}\right\rangle
$$

States with $J^{\pi}=0^{+}$occur only in the 7 representations with both $\lambda=$ even, $\mu=$ even. States with $J^{\pi}=2^{+}$can have both $\kappa=0$ and $\kappa=2$ in the 5 cases $(\lambda \mu) i=(14,2) i$, $(10,4) i,(82) i$, and occur in the remaining $15(\lambda \mu) i$ with $\kappa=1$ for $\mu$ odd, and $\kappa=0$ for $\mu=0$, leading to a basis of dimension 20 for $J^{\pi}=2^{+}$. The dimensions for $J^{\pi}=4^{+}$, $6^{+}$and $8^{+}$are 26,27 and 26.

Norm and overlap kernels for general binary-fragment systems. Similar techniques can be used to calculate the eigenvalues, $\Lambda_{Q(\lambda \mu) i}$, and the corresponding eigenvectors, $\left\langle\left[\left(\lambda_{c} \mu_{c}\right) \times(Q 0)\right](\lambda \mu) \mid(\lambda \mu) i\right\rangle$, for arbitrary $Q$, for any binary fragment decomposition 
of the $A$-particle system. With these numbers a norm or overlap kernel $\mathscr{K}\left(\boldsymbol{R}^{\prime \prime}, \boldsymbol{R}^{\prime}\right)$ can be evaluated in coordinate representation. Such a kernel can be expressed as [cf. eq. (8b)],

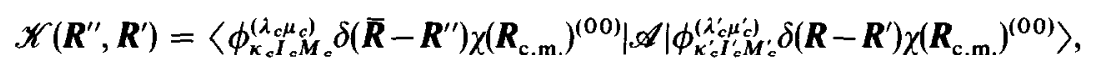

where the SU(3) coupled internal functions are themselves constructed from fragment internal functions of good SU(3) symmetry; e.g.,

$$
\begin{aligned}
& \phi_{\kappa_{c} I_{c} M_{c}}^{\left(\lambda_{c} \mu_{c}\right)}=\sum_{\kappa_{f} I_{f} I_{\kappa_{A-f} I_{A-f}}}\left\langle\left(\lambda_{f} \mu_{f}\right) \kappa_{f} I_{f} ;\left(\lambda_{A-f} \mu_{A-f}\right) \kappa_{A-f} I_{A-f} \|\left(\lambda_{c} \mu_{c}\right) \kappa_{c} I_{c}\right\rangle \\
& \times\left(\phi_{\kappa_{f} I_{f}}^{\left(\lambda_{f} \mu_{f}\right)} \times \phi_{\kappa_{A-\mathrm{f}} I_{A-\mathrm{f}}}^{\left(\lambda_{A-f} \mu_{A-f}\right)}\right)_{I_{c} M_{i}} .
\end{aligned}
$$

In eq. (107) the round bracket denotes ordinary angular momentum coupling. To evaluate the kernels in terms of the oscillator matrix elements of the type calculated in this section, it is necessary to expand the $\delta$-functions of eq. (106) in terms of 3-dimensional oscillator functions

$$
\delta\left(\overline{\boldsymbol{R}}-\boldsymbol{R}^{\prime \prime}\right)=\sum_{Q L M}\left(\chi(\overline{\boldsymbol{R}})_{L M}^{(Q 0)}\right)^{*} \chi\left(\boldsymbol{R}^{\prime \prime}\right)_{L M}^{(Q 0)}
$$

With this relation and straightforward SU(3) coupling and recoupling transformations of the type used throughout this investigation, cq. (106) can be put in the form

$$
\begin{aligned}
\mathscr{K}\left(\boldsymbol{R}^{\prime \prime}, \boldsymbol{R}^{\prime}\right)= & \sum_{Q(\lambda \mu)} \sum_{\left(\lambda_{0} \mu_{0}\right)}\left[\frac{d(\lambda \mu)}{d\left(\lambda_{c}^{\prime} \mu_{c}^{\prime}\right)}\right]^{\frac{1}{2}}(-1)^{Q+\lambda+\mu+\lambda_{c}^{\prime}+\mu_{c}^{\prime}} \\
& \left.\times \sum_{i}\left\langle\left[\left(\lambda_{c} \mu_{c}\right) \times(Q 0)\right](\lambda \mu)\right](\lambda \mu) i\right\rangle \Lambda_{Q(\lambda \mu) i}\left\langle(\lambda \mu) i \mid\left[\left(\lambda_{c}^{\prime} \mu_{c}^{\prime}\right) \times\left(Q^{\prime} 0\right)\right](\lambda \mu)\right\rangle \\
& \times \sum_{\rho} U\left(\left(\lambda_{c} \mu_{c}\right)(Q 0)\left(\lambda_{c}^{\prime} \mu_{c}^{\prime}\right)\left(0 Q^{\prime}\right) ;(\lambda \mu)_{--} ;\left(\lambda_{0} \mu_{0}\right)_{-} \rho\right) \\
& \times \sum_{\kappa_{0} L_{0}}\left\langle\left(\lambda_{c} \mu_{c}\right) \kappa_{c} I_{c} ;\left(\lambda_{0} \mu_{0}\right) \kappa_{0} L_{0} \|\left(\lambda_{c}^{\prime} \mu_{c}^{\prime}\right) \kappa_{c}^{\prime} I_{c}^{\prime}\right\rangle_{\rho} \\
& \times\left\langle I_{c} M_{c} L_{0} M_{0} \mid I_{c}^{\prime} M_{c}^{\prime}\right\rangle\left[\chi\left(R^{\prime \prime}\right)^{(Q 0)} \times \chi\left(R^{\prime}\right)^{\left(0 Q^{\prime}\right)}\right]_{\kappa_{0} L_{0} M_{0}}^{\left(\lambda_{0} \mu_{0}\right)}
\end{aligned}
$$

where $Q-Q^{\prime}$ is fixed: $Q-Q^{\prime}=\Delta$, cf. eq. (38); and where

$$
\begin{aligned}
& {\left[\chi\left(\boldsymbol{R}^{\prime \prime}\right)^{\left(Q^{0}\right)} \times \chi\left(\boldsymbol{R}^{\prime}\right)^{\left(0 Q^{\prime}\right)}\right]_{\kappa_{v} L_{u} M_{0}}^{\left(\lambda_{0} \mu_{0}\right)}=\sum_{L L^{\prime}}\left\langle(Q 0) L ;\left(0 Q^{\prime}\right) L^{\prime} \mid\left(\lambda_{0} \mu_{0}\right) \kappa_{0} L_{0}\right\rangle \mathscr{R}\left(R^{\prime \prime}\right)_{L}^{(Q 0)} \mathscr{R}\left(R^{\prime}\right)_{L^{\prime}}^{\left(Q^{\prime} 0\right)}} \\
& \times\left[\frac{\left(2 L^{\prime}+1\right)}{4 \pi}\right]^{\frac{1}{2}}(-1)^{L^{\prime}}\left\langle L M_{0} L^{\prime} 0 \mid L_{0} M_{0}\right\rangle Y_{L M_{0}}\left(\hat{\boldsymbol{R}}^{\prime \prime}, \hat{\boldsymbol{R}}^{\prime}\right)
\end{aligned}
$$

These are the generalizations of eqs. (9a) and (9b), where $\mathscr{R}(R)_{L}^{\left(Q^{0)}\right)}$ are again normalized radial harmonic oscillator functions, and the angular dependence is expressed through the spherical harmonic $Y_{L M_{0}}$ of the angular coordinates of the unit vector 
$\hat{\boldsymbol{R}}^{\prime \prime}$ relative to $\hat{\boldsymbol{R}}$. Eqs. (109) can be used to calculate both norm and overlap kernels. For the former, $\Delta=0$. For the latter, the functions $\phi^{\left(\lambda_{c} \mu_{c}\right)}$ are built from internal functions for fragments $f$ and $A-f$ through eq. (107) while, $\phi^{\left(\lambda_{c}^{\prime} \mu_{c}^{\prime}\right)}$ must be built by coupling internal functions for different fragments, $f^{\prime}$ and $A-f^{\prime}$.

${ }^{12} \mathrm{C}+{ }^{12} \mathrm{C}$ overlaps with channels $\mathrm{c}^{\prime}$. Overlaps between the ${ }^{12} \mathrm{C}+{ }^{12} \mathrm{C}$ cluster functions $\left|\left[\left(\lambda_{c} \mu_{c}\right)(Q 0)\right](\lambda \mu) \alpha\right\rangle$ and cluster functions with different fragment decompositions in channels $c^{\prime}$ are calculated by techniques similar to those used in connection with eqs. (101)-(104).

For the $\alpha+{ }^{20} \mathrm{Ne}$ channels with $\left.\phi\left({ }^{20} \mathrm{Ne}\right)\right)^{\left(\lambda_{c}^{\prime} \mu_{c}^{\prime}\right)}=\phi\left({ }^{20} \mathrm{Ne}\right)^{(80)}$, the overlap matrix elements

$$
\begin{aligned}
\left\langle\left[\left[\phi\left({ }^{12} \mathrm{C}\right)^{(04)} \times \phi\left({ }^{12} \mathrm{C}\right)^{(04)}\right]^{\left(\lambda_{c} \mu_{c}\right)}\right.\right. & \left.\times \chi\left(\overline{\boldsymbol{R}}_{12-12}\right)^{(Q+4,0)}\right]_{\alpha}^{(\lambda \mu)} \mid \\
& \times \mathscr{A}\left|\left[\phi(\alpha)^{(00)} \phi\left({ }^{20} \mathrm{Ne}\right)^{(80)} \times \chi\left(\boldsymbol{R}_{4-20}\right)^{(00)}\right]_{\alpha}^{(\lambda \mu)}\right\rangle
\end{aligned}
$$

for positive-parity states are obtained from the BS transform, which in analogy with eq. (101a), is obtained via the single-column functions $H\left({ }_{3}^{3\left(\lambda_{c} \mu_{c}\right) 1(20)}\right)$ by

$$
\begin{aligned}
H\left(\overline{\mathbf{K}}, \boldsymbol{K}^{*}\right)_{+}=\frac{1}{\sqrt{2}} \frac{4}{4 ! \sqrt{8 !}} & \exp \left\{\left[\frac{f^{\prime}(A-f)}{f\left(A-f^{\prime}\right)}\right]^{\frac{1}{2}}\left(\overline{\mathbf{K}} \cdot \boldsymbol{K}^{*}\right)\right\}\left\{\left[H\left({ }_{3}^{(302) 1(20)}\right)\right]^{4}\right. \\
+ & \left.6\left[H\left({ }_{3}^{3(02) 1(20)}\right)\right]_{5}^{2}\left[H\left({ }_{3}^{3(10) 1(20)}\right)\right]^{2}+\left[H\left({ }_{3}^{3(10) 1(20)}\right)\right]^{4}\right\} .
\end{aligned}
$$

Expansions in terms of functions $\mathscr{P}^{\left(\lambda_{c} \mu_{c}\right)(80)}$ with $\left(\lambda_{c} \mu_{c}\right)==(08),(24)$, and (40), [cf. eq. (101b)], lead to the desired overlap matrix elements. These expansions follow from the three relations, eqs. (112)-(114), below. With $f=12, f^{\prime}=4$ :

$$
\begin{aligned}
\frac{1}{\sqrt{2}} \frac{4}{4 ! \sqrt{8 !}} \exp & \left\{\left[\frac{f^{\prime}(A-f)}{f\left(A-f^{\prime}\right)}\right]^{\frac{1}{2}}\left(\bar{K} \cdot K^{*}\right)\right\}\left[H\left({ }_{3}^{3(02) 1(20)}\right)\right]^{4} \\
& =\sum_{\left(\lambda_{c} \mu_{c}\right)=(08)(24)(40)} \sum_{Q\left(\lambda_{\mu}\right)}(-1)^{Q+\lambda+\mu}\left[\frac{5 d(80) d(Q 0) d\left(\lambda_{c} \mu_{c}\right)}{2 d(\lambda \mu)[d(40)]^{2}}\right]^{\frac{1}{2}} \frac{[Q !(Q+4) !]^{\frac{1}{2}}}{4(Q-4) !} \\
& \times \sum_{p=0}^{f^{\prime}=4}(-1)^{p}\left(\begin{array}{l}
4 \\
p
\end{array}\right)\left(\frac{A}{f(A-f)}\right)^{4}\left(\frac{A}{f^{\prime}\left(A-f^{\prime}\right)}\right)^{2}\left[\frac{f^{\prime}(A-f)-p A}{\left[f(A-f) f^{\prime}\left(A-f^{\prime}\right)\right]^{\frac{1}{2}}}\right]^{Q-4} \\
& \times\left\{16 \delta_{\left(\lambda_{c} \mu_{c}\right)(08)}+2 \delta_{\left(\lambda_{c} \mu_{c}\right)(24)}+1 \delta_{\left(\lambda_{c} \mu_{c}\right)(40)}\right\} \\
& \times U\left(\left(\lambda_{c} \mu_{c}\right)(40)(44)(40) ;(04)(80)\right) U((80)(Q 0)(44)(0, Q-4) ;(\lambda \mu)(40)) \\
& \times U\left(\left(\lambda_{c} \mu_{c}\right)(80)(\lambda \mu)(Q-4,0) ;(44)(Q+4,0)\right) \operatorname{Pa}^{\left(\lambda_{c} \mu_{c}\right)(80)}
\end{aligned}
$$


while

$$
\begin{aligned}
& \frac{1}{\sqrt{2}} \frac{4}{4 ! \sqrt{8 !}} \exp \left\{\left[\frac{f^{\prime}(A-f)}{f\left(A-f^{\prime}\right)}\right]^{\frac{1}{2}}\left(\bar{K} \cdot K^{*}\right)\right\}\left[H\left({ }_{3}^{(30) 1(20)}\right)\right]^{4} \\
& =\sum_{Q(\lambda \mu)}(-1)^{Q+\lambda+\mu}\left[\frac{5 d(80) d(Q+4,0)}{2 d(40) d(\lambda \mu)}\right]^{\frac{1}{2}} \sum_{l j}^{f_{p}} \sum_{p=0}^{f^{\prime}=4} \frac{[Q !(Q+4) !]^{\frac{1}{2}}}{(Q-l) !} \frac{(-1)^{l+j}}{2^{l-2}} \\
& \times\left({ }_{i}^{4}\right)\left({ }_{j}^{4-l}\right)\left({ }_{p-j}{ }^{l}\right)\left(\frac{A}{f(A-f)}\right)^{2+l / 2}\left(\frac{A}{f^{\prime}\left(A-f^{\prime}\right)}\right)^{l / 2}\left[\frac{f^{\prime}(A-f)-p A}{\left[f(A-f) f^{\prime}\left(A-f^{\prime}\right)\right]^{\frac{1}{2}}}\right]^{Q-l} \\
& \times U((40)(Q+4,0)(80)(0 Q) ;(\lambda \mu)(40)) \mathscr{P}^{(40)(80)} \text {, } \\
& \frac{6}{\sqrt{2}} \frac{4}{4 ! \sqrt{8 !}} \exp \left\{\left[\frac{f^{\prime}(A-f)}{f\left(A-f^{\prime}\right)}\right]^{\frac{1}{2}}\left(\overline{\boldsymbol{K}} \cdot \boldsymbol{K}^{*}\right)\right\}\left[H\left({ }_{3}^{3(02) 1(20)}\right)\right]_{5}^{2}\left[H\left({ }_{3}^{3(10) 1(20)}\right)\right]^{2} \\
& =\sum_{\left(\lambda_{c} \mu_{c}\right)=(24)(40)} \sum_{Q(\lambda \mu)}(-1)^{Q+\lambda+\mu}\left[\frac{5 d(Q 0)}{2 d(\lambda \mu)}\right]_{l_{j}^{\frac{1}{2}}} \frac{(-1)^{l+j}}{2^{l}} \frac{[Q !(Q+4) !]^{\frac{1}{2}}}{(Q-l-2) !} \\
& \left.\times \sum_{p=0}^{f^{\prime}=4}\left({ }_{l}^{2}\right)\left(_{j}^{4-l}\right)()_{p-j}{ }^{l}\right)\left(\frac{A}{f(A-f)}\right)^{3+l / 2}\left(\frac{A}{f^{\prime}\left(A-f^{\prime}\right.}\right)^{1+l / 2}\left[\frac{f^{\prime}(A-f)-p A}{\left[f(A-f) f^{\prime}\left(A-f^{\prime}\right)\right]^{\frac{1}{2}}}\right]^{Q-l-2} \\
& \times\left\{4[33]^{\frac{1}{2}} \delta_{\left(\lambda_{c} \mu_{c}\right)(24)}+6 \delta_{\left(\lambda_{c} \mu_{c}\right)(40)}\right\} \\
& \times U((80)(Q 0)(62)(0, Q-2) ;(\lambda \mu)(20)) \\
& \times U\left(\left(\lambda_{c} \mu_{c}\right)(60)(\lambda \mu)(Q-2,0) ;(62)(Q+4,0)\right) \mathscr{P}^{\left(\lambda_{c} \mu_{c}\right)(80)} \text {. }
\end{aligned}
$$

Specific numerical values of SU(3) recoupling coefficients have been used, wherever possible, to simplify these expressions.

Similar expressions give the overlaps between the ${ }^{12} \mathrm{C}+{ }^{12} \mathrm{C}$ and ${ }^{8} \mathrm{Be}+{ }^{16} \mathrm{O}$ cluster decompositions.

Overlap matrix elements between positive-parity states of ${ }^{12} \mathrm{C}+{ }^{12} \mathrm{C}$ and the ${ }^{23} \mathrm{Na}+\mathrm{p}$ or ${ }^{23} \mathrm{Mg}+\mathrm{n}$ fragment decompositions are particularly simple. With $\phi^{\left(\lambda_{c}^{\prime} \mu_{c}^{\prime}\right)}=\phi^{(83)}$, the BS transform for this overlap is given by a single product of singlecolumn BS transforms

$H\left(\bar{K}, K^{*}\right)_{+}=\frac{1}{\sqrt{2}} \frac{1}{4 !} \frac{2^{6}}{[8 ! 6 !]^{\frac{1}{2}}}\left[\frac{7}{11}\right]^{\frac{1}{2}} \exp \left\{\left(\bar{K} \cdot K^{*}\right) /[23]^{\frac{1}{2}}\right\}\left[H\left(3_{6}^{3(02) 0(21)}\right)\right]^{3}\left[H\left(3_{3}^{3(02) 1(20)}\right)\right]^{1}$,

where the normalization factor now includes a spin-isospin factor, as well as factors needed to insure properly normalized ${ }^{12} \mathrm{C}+{ }^{12} \mathrm{C}$ and ${ }^{23} \mathrm{Na}+\mathrm{p}$ internal $\mathrm{K}$-space functions, (cf. table 5). An expansion in terms of the proper $\mathrm{SU}(3)$ coupled combina- 
tions of the $K$-space functions leads to the result

$$
\begin{aligned}
& \left\langle\left[\left[\phi\left({ }^{12} \mathrm{C}\right)^{(04)} \times\left({ }^{12} \mathrm{C}\right)^{(04)}\right]^{\left(\lambda_{c} \mu_{c}\right)} \times \chi\left(\bar{R}_{12-12}\right)^{(Q+10,0)}\right]_{\alpha}^{(\lambda \mu)}\right| \\
& \times \mathscr{A}\left|\left[\left[\phi\left(\xi_{n}\right) \times \phi(A=23)^{(83)}\right]^{(83)} \times \chi\left(R_{23-1}\right)^{(Q 0)}\right]_{a}^{(\lambda \mu)}\right\rangle \\
& =(-1)^{Q+\lambda+\mu}\left[1+(-1)^{Q}\right]\left[\frac{13 d(Q 0)}{30 d(Q-1,1)}\right]^{\frac{1}{2}} \frac{[Q !(Q+10) !]^{\frac{1}{2}}}{(Q-1) !} \\
& \times\left(\frac{A}{f(A-f)}\right)^{5} \frac{A(A-f)^{Q-1}}{[(A-1) f(A-f)]^{Q / 2}} \sum_{\left(\overline{\left.\bar{\lambda}_{c} \bar{\mu}_{c}\right)}\right.}\left\{16 \sqrt{\frac{1}{11}} \delta_{\left(\lambda_{c} \mu_{c}\right)(08)} \delta_{\left(\lambda_{c} \bar{\mu}_{e}\right)(07)}\right. \\
& \left.+\frac{1}{2} \sqrt{\frac{1}{55}} \delta_{\left.\left(\lambda_{c} \mu_{c}\right)(24)\right)}\left[7 \sqrt{6} \delta_{\left(\pi_{c} \bar{\mu}_{c}\right)(23)}-9 \delta_{\left(\lambda_{c} \bar{\mu}_{c}\right)(15)}\right]-\sqrt{\frac{3}{8}} \delta_{\left(\lambda_{c} \mu_{c}\right)(40)} \delta_{\left(\lambda_{c} \bar{\mu}_{c}\right)(31)}\right\} \\
& \times \sum_{\rho}\left[\begin{array}{llll}
(11,0) & \left(\bar{\lambda}_{c} \bar{\mu}_{c}\right) & (73) & - \\
(Q-1,0) & (01) & (Q-1,1) & - \\
(Q+10,0) & \left(\lambda_{c} \mu_{c}\right) & (\lambda \mu) & - \\
- & - & \rho &
\end{array}\right] \\
& \times U\left((37)(01)(\mu \lambda)(0 Q) ;(38)_{-} ;(1, Q-1)_{-} \rho\right) \text {, }
\end{aligned}
$$

where the contributions from the direct and the one-nucleon exchange term have been written out explicitly. (Note that $f=12, A=24$ ). In the ket the nucleon spin-isospin function $\phi\left(\xi_{n}\right)$ is coupled with the $A=23$ internal function to a resultant SU(4) scalar function of SU(3) symmetry (83).

For the $\alpha+{ }^{20} \mathrm{Ne}$ channels with $\phi\left({ }^{20} \mathrm{Ne}\right)^{\left(\lambda_{c} \mu_{c}\right)}=\phi\left({ }^{20} \mathrm{Ne}\right){ }^{(82)}$ with $I_{A-f^{\prime}}=2^{-}$, $3^{-}, \ldots$, the internal ${ }^{20} \mathrm{Ne}$ wave functions can be expressed simply in terms of the singleshell-model component $\left.{ }^{54}\right),\left|\mathrm{s}^{4}\left[\mathrm{p}^{11}(01)(\mathrm{sd})^{5}(81)\right](82) \kappa=2 J M_{J}\right\rangle$, so that the techniques of sect. 3 can be applied with no essential modification. The needed single-column BS transforms are included in the tabulation of appendix C. The full BS transform for this overlap then has the form

$$
\begin{aligned}
& H\left(\bar{K}, K^{*}\right)_{+}=\sqrt{ } \frac{1}{2} \frac{1}{4 !} \frac{8}{[5 \times 7 \times 6 !]^{\frac{1}{2}}} \mathrm{e}^{\left(\overline{\boldsymbol{K}} \cdot \mathrm{K}^{*}\right) /[5] \frac{1}{4}}\left\{\left[H\left(3_{5}^{3(02) 1(20)}\right)\right]^{3}\left[H\left({ }_{3}^{3(02) 1(22)}\right)\right]^{1}\right. \\
& +3\left[H\left({ }_{3}^{3(02) 1(20)}\right)\right]^{2}\left[H\left({ }_{3}^{3(10) 1(20)}\right]_{5}^{1}\left[H\left({ }_{3}^{3(10) 1(22)}\right)\right]^{1}\right. \\
& +3\left[H\left(3_{3}^{3(02) 1(20)}\right)\right]^{1}\left[H\left(3_{3}^{3(10) 1(20)}\right)\right]^{2}\left[H\left(3_{3}^{3(02) 1(22)}\right)\right]^{1} \\
& \left.+\left[H\left({ }_{3}^{3(10) 1(20)}\right)\right]^{3}\left[H\left({ }_{3}^{3(10) 1(22)}\right)\right]^{1}\right\} \text {. }
\end{aligned}
$$

Despite the seeming complexity of this transform, the expansion in terms of $K$-space functions $\mathscr{T}^{\left(\lambda_{c} \mu_{c}(82)\right.}$, cf. eq. (101b), can be carried out quite explicitly. As for eq. (116), sums over exchange terms can be carried out, and specific numerical values of the SU(3) recoupling coefficients can be used to reduce the final expression for the overlap matrix elements to one which depends only on simple $Q,(\lambda \mu)$ dependent Racah coefficients. 
$\left\langle\left[\left[\phi\left({ }^{12} \mathrm{C}\right)^{(04)} \times \phi\left({ }^{12} \mathrm{C}\right)^{(04)}\right]^{\left(\lambda_{c} \mu_{c}\right)} \times \chi\left(\bar{R}_{12-12}\right)^{(Q+5,0)}\right]_{\alpha}^{(\lambda \mu)}\right|$

$\left.\left.\times \mathscr{A} \mid\left[\phi(\alpha)^{(00)} \times \phi\left({ }^{20} \mathrm{Ne}\right)^{(82)}\right]^{(82)} \times \chi\left(R_{4-20}\right)^{(00)}\right]_{z}^{(2 \mu)}\right\rangle$

$=\frac{[Q !(Q+5) !]^{\frac{1}{2}}}{(2 \sqrt{ } 5)^{Q-3}}\left[1+(-1)^{Q-1}\right]\left[2^{Q-3}-4\right] \frac{(Q-7)}{(Q-3) !}$

$\times\left\{\sum_{(\bar{\lambda} \bar{\mu})}\left(2^{8^{-} \cdot 3^{2} \cdot \frac{1}{5}}\left[\frac{13}{14}\right]^{\frac{1}{1}} \delta_{\left(\lambda_{c} \mu_{c}\right)(08)} \delta_{(\bar{\lambda} \bar{\mu})(55)}-\frac{1}{2^{9} \cdot 5 \cdot 7 \cdot[22]^{\frac{1}{2}}} \delta_{\left(\lambda_{c} \mu_{c}\right)(40)} \delta_{(\bar{\lambda} \bar{\mu})(63)}\right.\right.$

$+\delta_{\left(\lambda_{c} \mu_{c}\right)(24)}\left[\frac{1}{2^{10} \cdot 3^{2} \cdot 5}\left[\frac{13}{35}\right]^{\frac{1}{2}} \delta_{(\bar{\lambda} \bar{\mu})(55)}-\frac{1}{2^{9} \cdot 5^{2} \cdot 7}\left[\frac{143}{15}\right]^{\frac{1}{2}} \delta_{(\bar{\lambda} \bar{\mu})(63)}+\frac{1}{2^{11} \cdot 5^{2}}\right.$

$\left.\left.\times\left[\frac{143}{21}\right]^{\frac{1}{2}} \delta_{(\bar{\lambda} \bar{\mu})(71)}\right]\right)$

$\left.\times U\left((Q-3,0)(80)(\lambda \mu)\left(\lambda_{c} \mu_{c}\right) ;(Q+5,0)(\bar{\lambda} \bar{\mu})\right) U((Q-3,0)(30)(\lambda \mu)(82) ;(Q 0)(\bar{\lambda} \bar{\mu}))\right\}$

$-\frac{[Q !(Q+5) !]^{\frac{1}{2}}}{(2 \sqrt{5})^{Q-3}}\left[1+(-1)^{Q-1}\right]\left[2^{Q-3}-4\right] \frac{1}{(Q-4) !}$

$\times\left\{\frac{[13]^{\frac{1}{2}} \delta_{\left(\lambda_{c} \mu_{c}\right)(08)}}{2^{6} \cdot 3 \cdot 5[2 \cdot 3 \cdot 5 \cdot 7 \cdot 11]^{\frac{1}{2}}}+\frac{[13]^{\frac{1}{2}} \delta_{\left(\lambda_{c} \mu_{c}\right)(24)}}{2^{7} \cdot 3^{2} \cdot 5[2 \cdot 5 \cdot 7 \cdot 11]^{\frac{1}{2}}}+\frac{\delta_{\left(\lambda_{c} \mu_{c}\right)(40)}}{2^{9} \cdot 3 \cdot 5[2 \cdot 3 \cdot 5 \cdot 11]^{\frac{1}{2}}}\right\}$

$\times U\left((Q-4,0)(90)(\lambda \mu)\left(\lambda_{c} \mu_{c}\right) ;(Q+5,0)(54)\right) U((Q-4,0)(40)(\lambda \mu)(82) ;(Q 0)(54))$

$-\frac{[Q !(Q+5) !]^{\frac{2}{2}}}{(2 \sqrt{5})^{Q-1}}\left[1+(-1)^{Q-1}\right]\left\{\frac{\left[2^{Q-1}-4\right]}{(Q-1) !}-\frac{3\left[2^{Q-2}-2\right]}{2}+\right.$

$\left.+\frac{3}{4} \frac{2^{Q-3}}{(Q-3) !}-\frac{1}{8} \frac{\left[2^{Q-4}+2\right]}{(Q-4) !}\right\}$

$\times\left\{\sum_{(\bar{\lambda} \mu)}\left[\frac{[13]^{\frac{1}{2}}}{2^{5} \cdot 3[3]^{\frac{1}{2}}} \delta_{\left(\lambda_{c} \mu_{c}\right)(24)} \delta_{(\bar{\lambda} \bar{\mu})(73)}-\frac{1}{2^{3} \cdot 3^{2}}\left[\frac{5}{22}\right]^{\frac{1}{2}} \delta_{\left(\lambda_{c} \mu_{c}\right)(40)} \delta_{(\bar{\lambda} \bar{\mu})(81)}\right]\right.$

$\left.\times U\left((Q-1,0)(60)(\lambda \mu)\left(\lambda_{c} \mu_{c}\right) ;(Q+5,0)(\bar{\lambda} \bar{\mu})\right) U((Q-1,0)(10)(\lambda \mu)(82) ;(Q 0)(\bar{\lambda} \bar{\mu}))\right\}$

$-\frac{[Q !(Q+5) !]^{\frac{1}{2}}}{(2 \sqrt{5})^{Q-1}}\left[1+(-1)^{Q-1}\right]\left\{\frac{\left[2^{Q-2}-2\right]}{(Q-2) !}-\frac{2^{Q-3}}{(Q-3) !}+\frac{1\left[2^{Q-4}+2\right]}{4} \frac{(Q-4) !}{(Q)}\right.$

$\times\left\{\frac{1}{2^{3} \cdot 3}\left[\frac{13}{3 \cdot 7 \cdot 11}\right]^{\frac{1}{2}} \delta_{\left(\lambda_{c} \mu_{c}\right)(24)}+\frac{1}{2^{5} \cdot 3}\left[\frac{5}{77}\right]^{\frac{1}{2}} \delta_{\left(\lambda_{c} \mu_{c}\right)(40)}\right\}$

$\times U\left((Q-2,0)(70)(\lambda \mu)\left(\lambda_{c} \mu_{c}\right) ;(Q+5,0)(72)\right) U((Q-2,0)(20)(\lambda \mu)(82) ;(Q 0)(72))$.

Spectroscopic amplitudes, $A_{c^{\prime} i}$. Overlap matrix elements such as those given by eqs. (116) or (118) can now be used to calculate spectroscopic amplitudes for the ${ }^{12} \mathrm{C}+$ ${ }^{12} \mathrm{C}$ quasi-bound states to the most important observed exit channels. In this work no 
attempt is made to solve the quasi-bound-state problem. Instead, we calculate spectroscopic amplitudes $A_{c^{\prime} i}$ for the "molecular" basis $|i\rangle,\left(=\left|(\lambda \mu) i \kappa J M_{J}\right\rangle\right.$ of table 6). These may serve as a diagnostic tool since the relative magnitudes of $A_{c^{\prime} i}$ for various observed channels $c^{\prime}$ may give an indication of the importance of a particular $|i\rangle$ in a particular fine structure peak. The amplitude $A_{c^{\prime} i}$ is given by the projection of the normalized, fully antisymmetrized state $|i\rangle$ on the state $\left|c^{\prime}\right\rangle$ which is specified by the fragment decomposition $f^{\prime}+A-f^{\prime}$ and the angular momenta $I_{f^{\prime}}, I_{A-f^{\prime}}, I_{c^{\prime}}$, and $L$, appropriate to channel $c^{\prime}$. The spectroscopic amplitudes depend on three types of factors: (i) the amplitudes and normalization factors $\left(\Lambda_{i}\right)^{-\frac{1}{2}}$ of table 6 which define the states $|i\rangle$, (ii) angular momentum independent overlap matrix elements, such as those given by eqs. (116) or (118) and (iii) the factors which carry the angular momentum dependence. These are made up of $S U(3) \supset R(3)$ Wigner coefficients, and, in some cases, ordinary angular momentum recoupling coefficients. The angular momentum structure is somewhat different in the three cases.

(i) In the ${ }^{12} \mathrm{C}+{ }^{12} \mathrm{C}$ channels both $I_{f^{\prime}}$, and $I_{A-f^{\prime}}$ may be different from zero. Their resultant $I_{c^{\prime}}$ is coupled with $L$, the orbital angular momentum of the relative motion function to resultant $J: I_{f^{\prime}}+I_{A-f^{\prime}}=I_{c^{\prime}}, I_{c^{\prime}}+L=J$.

(ii) In the $\alpha+{ }^{20} \mathrm{Ne}$ or ${ }^{16} \mathrm{O}+{ }^{8} \mathrm{Be}$ channels, $I_{f^{\prime}}=0$, and $I_{A-f}$, may be different from zero. The coupling is $I_{A-f^{\prime}}+L=J$.

(iii) In the ${ }^{23} \mathrm{Na}+\mathrm{p}$ (or ${ }^{23} \mathrm{Mg}+\mathrm{n}$ ) channels, the nucleon spin, $s=\frac{1}{2}$, is coupled with the orbital angular momentum, $l$, of the nucleon-nucleus relative motion function to resultant $j$.

The $A-f^{\prime}=23$ states are built from the angular momentum coupled states $\left|\left(\lambda_{A-f^{\prime}}, \mu_{A-f^{\prime}}\right) \kappa_{L}\left(L_{A}-f^{\prime} S_{A-f^{\prime}}\right) I_{A-f^{\prime}}\right\rangle$, with $S_{A-f^{\prime}}=\frac{1}{2}$. Here $\kappa_{L}$ is the orthonormalized $K_{L},(L$-type $K)$, yuantum number $\left.{ }^{35}\right)$. The rotational bands in ${ }^{23} \mathrm{Na}$ and ${ }^{23} \mathrm{Mg}$ are assumed to have good $J$-type $K$-quantum numbers or their orthonormalized $\kappa_{I^{\prime}}$ analogue, with $\kappa_{r^{\prime}}=\frac{3}{2}$ and $\frac{1}{2}$, in particular. The transformation to a $\kappa_{r^{\prime}}$ basis is given by

$$
\left|\left(\lambda_{A-f^{\prime}} \mu_{A-f^{\prime}}\right) \kappa_{I^{\prime}} S_{A-f^{\prime}} I_{A-f^{\prime}} M_{A-f^{\prime}}\right\rangle=\sum_{\kappa^{\prime} L_{L} L_{A-f^{\prime}}} c_{\kappa^{\prime} L L_{A-f^{\prime}}} \mid\left(\lambda_{A-f^{\prime}}, \mu_{A-f^{\prime}}\right) \kappa_{L}^{\prime}\left(L_{A-f^{\prime}}, S_{A-f^{\prime}}\right)
$$

where the coefficients $c_{\kappa \ell L}$ can be evaluated by the use of eqs. (3.2) to (3.6) of ref. ${ }^{55}$ ).

For a specific ${ }^{12} \mathrm{C}+{ }^{12} \mathrm{C}$ channel, $\left(I_{f^{\prime}} I_{A-f^{\prime}}\right) I_{c^{\prime}}$, the spectroscopic amplitude $A_{c^{\prime} i}$ is given by the overlap of the $Q=14$ component of the ${ }^{12} \mathrm{C}+{ }^{12} \mathrm{C}$ function

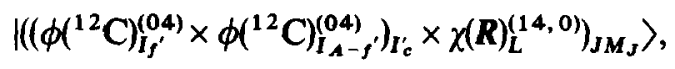

with the state $\left.\Lambda_{i}^{-\frac{1}{2}} d i\right\rangle$. This gives the amplitude

$$
\begin{aligned}
A_{\mathrm{L}}\left((\lambda \mu) i \kappa J \rightarrow\left({ }^{12} \mathrm{C}\left(I_{f^{\prime}}\right) \times{ }^{12} \mathrm{C}\left(I_{A}-f^{\prime}\right)\right)_{I_{\mathrm{c}}^{\prime}}\right) \\
\quad=\sum_{\left(\lambda_{\mathrm{c}}^{\prime} \mu^{\prime}\right) \kappa_{\mathrm{c}}^{\prime}}\left\langle(04) I_{f^{\prime}} ;(04) I_{A-f^{\prime}} \|\left(\lambda_{\mathrm{c}}^{\prime} \mu_{\mathrm{c}}^{\prime}\right) \kappa_{\mathrm{c}}^{\prime} I_{c}^{\prime}\right\rangle\left\langle\left(\lambda_{c}^{\prime} \mu_{c}^{\prime}\right) \kappa_{\mathrm{c}}^{\prime} I_{\mathrm{c}}^{\prime} ;(14,0) L \|(\lambda \mu) \kappa J\right\rangle
\end{aligned}
$$




$$
\begin{aligned}
& \times \sum_{\left(\lambda_{c} \mu_{c}\right)} \frac{1}{A_{i}^{\frac{1}{2}}}\left\langle\left[\left(\lambda_{c} \mu_{c}\right) \times(14,0)\right](\lambda \mu) \mid(\lambda \mu) i\right\rangle \\
& \times\left\langle\left[\left[\phi\left({ }^{12} \mathrm{C}\right)^{(04)} \times \phi\left({ }^{12} \mathrm{C}\right)^{(04)}\right]^{\left(\lambda^{\prime} \mu_{c}^{\prime}\right)} \times \chi(R)^{(14,0)}\right]_{\alpha}^{(\lambda \mu)}\right| \\
& \left.\left.\times \mathscr{A} \mid\left[\phi\left({ }^{12} \mathrm{C}\right)^{(04)} \times \phi\left({ }^{12} \mathrm{C}\right)^{(04)}\right]^{\left(\lambda_{c} \mu_{c}\right)} \times \chi(R)^{(14,0)}\right]_{\alpha}^{(\lambda \mu)}\right\rangle,
\end{aligned}
$$

where the double-barred coefficients are $\mathrm{SU}(3) \supset \mathrm{R}(3)$ Wigner coefficients, and the $i$-dependent factors can be read from table 6. With $I_{f^{\prime}}=I_{A-f^{\prime}}=I_{c^{\prime}}=0$, this gives the amplitudes, $A_{c i}$, for the entrance channel. These amplitudes are shown in table 7

TABLE 7

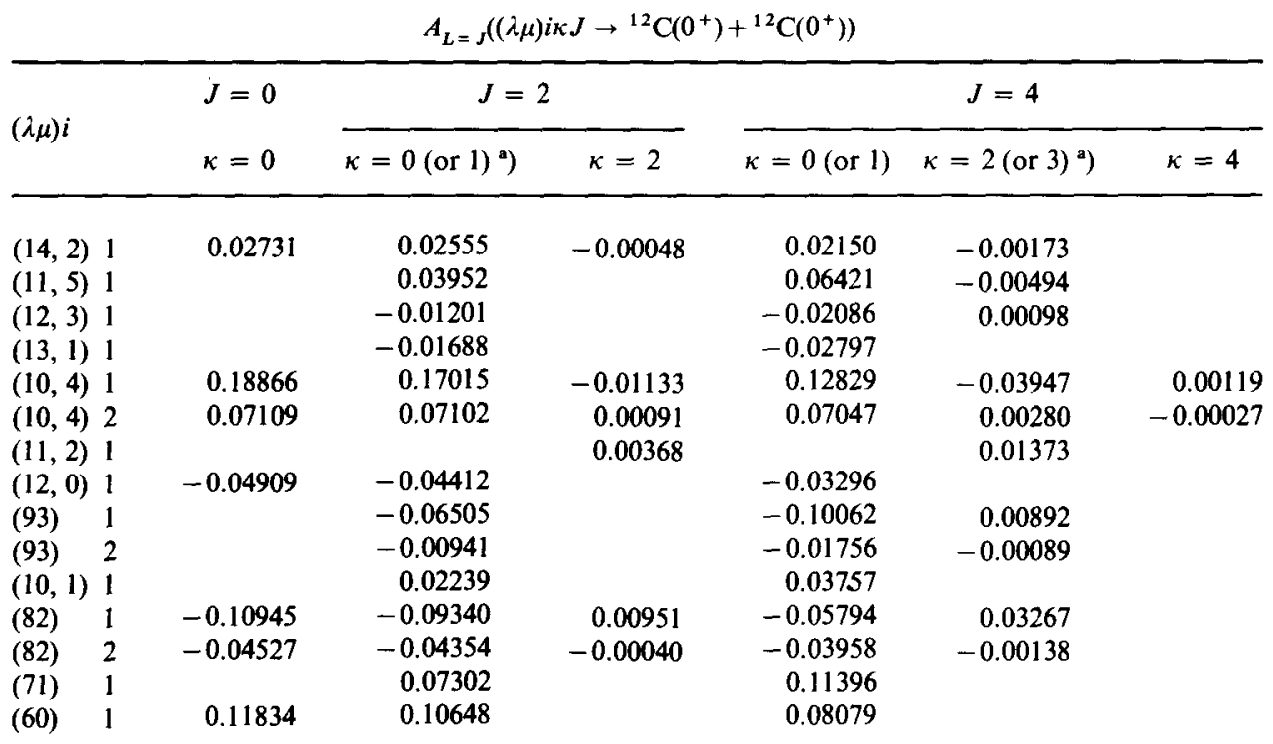

a) $\kappa=0,2, \ldots$ for states with $\mu=$ even $\kappa=1,3, \ldots$ for states with $\mu=$ odd.

for all states $|i\rangle \equiv|(\lambda \mu) i \kappa J M\rangle$ with $J=0,2,4$. We note the following properties of these amplitudes:

(i) Only states with $\kappa=0$ ( $\mu=$ even) and $\kappa=1$ ( $\mu=$ odd) have significant amplitudes. Amplitudes for states with $\kappa \geqq 2$ are so small that such states cannot be expected to play a role in the ${ }^{12} \mathrm{C}+{ }^{12} \mathrm{C}$ resonances.

(ii) The few most significant amplitudes are those for states with $\lambda=$ even, $\mu=$ even.

(iii) The large amplitudes for the $\kappa=0$ band of states in a given $(\lambda \mu)$ are only very mild functions of $J$.

For exit channels $c^{\prime}$, such as the $\alpha+{ }^{20} \mathrm{Ne}$ or ${ }^{16} \mathrm{O}+{ }^{8} \mathrm{Be}$ channels, with $\left(\lambda_{f^{\prime}}, \mu_{f^{\prime}}\right)=$ 
$(00), I_{f^{\prime}}=0, S_{f^{\prime}}=0,\left(\lambda_{A-f^{\prime}} \mu_{A-f^{\prime}}\right)=\left(\lambda_{c}^{\prime} \mu_{c}^{\prime}\right)$,

$$
\begin{aligned}
& A_{\mathrm{L}}\left((\lambda \mu) i \kappa J \rightarrow f^{\prime}+A-f^{\prime}\left(\lambda_{c}^{\prime} \mu_{c}^{\prime}\right) I_{c^{\prime}}\right) \\
& \quad=\sum_{\left(\lambda_{c} \mu_{c}\right)=(08)(24)(40)} \frac{1}{A_{i}^{\frac{1}{i}}}\left\langle\left[\left(\lambda_{c} \mu_{c}\right) \times(14,0)\right](\lambda \mu) \mid(\lambda \mu) i\right\rangle\left\langle\left(\lambda_{c}^{\prime} \mu_{c}^{\prime}\right) \kappa_{c}^{\prime} I_{c^{\prime}} ;(14-\Delta, 0) L \|(\lambda \mu) \kappa J\right\rangle \\
& \quad \times\left\langle\left[\left[\phi\left({ }^{12} \mathrm{C}\right)^{(04)} \times \phi\left(^{12} \mathrm{C}\right)^{(04)}\right]^{\left(\lambda_{c} \mu_{c}\right)} \times \chi\left(\bar{R}_{12-12}\right)^{(14,0)}\right]_{\alpha}^{(\lambda \mu)}\right| \\
& \quad \times \mathscr{A} \mid\left[\left[\phi_{f^{\prime}}^{(00)} \times \phi_{A}^{\left(\lambda_{c}^{\prime} \mu_{c}^{\prime}\right)}\right]^{\left(\lambda_{c}^{\prime} \mu_{c}^{\prime}\right)} \times \chi\left(R_{f^{\prime}-\left(A-f^{\prime}\right)}^{(14-\Delta, 0)}\right]_{\alpha}^{(\lambda \mu)}\right\rangle
\end{aligned}
$$

where, $\Delta=4$, for $\phi\left({ }^{20} \mathrm{Ne}\right)^{\left(\lambda_{c}^{\prime} \mu_{c}^{\prime}\right)}=\phi\left({ }^{20} \mathrm{Ne}\right)^{(80)}$; and $\Delta=5$ for $\phi\left({ }^{20} \mathrm{Ne}\right)^{\left(\lambda_{c}^{\prime} \mu_{c}^{\prime}\right)}=$ $\phi\left({ }^{20} \mathrm{Ne}\right){ }^{(82)}$, e.g.

In the $\mathrm{p}+{ }^{23} \mathrm{Na}$ and $\mathrm{n}+{ }^{23} \mathrm{Mg}$ channels, the spin of the nucleon, $s=\frac{1}{2}$, is coupled to the $l$ of the nucleon- $A=23$ nucleus relative motion function, $l+s=j$, to give the amplitudes

$$
\begin{aligned}
& A_{l s j}\left((\lambda \mu) i \kappa J \rightarrow\left(\lambda_{A-f^{\prime}}, \mu_{A-f^{\prime}}\right) \kappa_{I^{\prime}} I_{A-f^{\prime}}\right) \\
& =\sum_{\left(\lambda_{c} \mu_{c}\right)=(08)(24)(40)} \frac{1}{\Lambda_{i}^{\frac{1}{i}}}\left\langle\left[\left(\lambda_{c} \mu_{c}\right) \times(14,0)\right](\lambda \mu) \mid(\lambda \mu) i\right\rangle \\
& \times \sum_{\kappa \dot{L} L_{A}-f^{\prime}} c_{\kappa \dot{L} L_{A-f^{\prime}}}\left\langle\left(\lambda_{A-f^{\prime}} \mu_{A-f^{\prime}}\right) \kappa_{L}^{\prime} L_{A-f^{\prime}} ;(40) l \mid(\lambda \mu) \kappa J\right\rangle \\
& \times\left( \pm \sqrt{\frac{1}{2}}\right)\left[\frac{\left(2 I_{A-f^{\prime}}+1\right)}{(2 s+1)\left(2 L_{A-f^{\prime}}+1\right)}\right]^{\frac{1}{2}} U\left(I_{A-f^{\prime}} s J l ; L_{A-f^{\prime}} j\right) \\
& \times\left\langle\left[\left[\phi\left({ }^{12} \mathrm{C}\right)^{(04)} \times \phi\left({ }^{12} \mathrm{C}\right)^{(04)}\right]^{\left(\lambda_{c} \mu_{c}\right)} \times \chi\left(\bar{R}_{12-12}\right)^{(14,0)}\right]_{\alpha}^{(\lambda \mu)}\right| \\
& \times \mathscr{A}\left|\left[\left[\phi\left(\xi_{n}\right) \times \phi^{\left(\lambda_{A}-f^{\prime} \mu_{A-f^{\prime}}\right)}\right]^{\left(\lambda_{A}-f^{\prime} \mu_{A}-f^{\prime}\right)} \times \chi\left(\boldsymbol{R}_{23-1}\right)^{(40)}\right]_{\alpha}^{(\lambda \mu)}\right\rangle,
\end{aligned}
$$

where the states of ${ }^{23} \mathrm{Na}$ (or ${ }^{23} \mathrm{Mg}$ ) are approximated by the states defined in eq. (119) with $S_{A-f^{\prime}}=s=\frac{1}{2}$. The isospin Clebsch-Gordan coefficient, $\left( \pm \sqrt{\frac{1}{2}}\right)$, applies to the cases $\mathrm{n}+{ }^{23} \mathrm{Mg}$ and $\mathrm{p}+{ }^{23} \mathrm{Na}$, respectively.

Apart from the angular momentum dependent factors, the magnitudes of the spectroscopic amplitudes (121), (122), are determined by

$$
\begin{aligned}
\sum_{\left(\lambda_{c} \mu_{c}\right)} & =(08)(25)(40) \\
& \frac{1}{A_{i}^{t}}\left\langle\left[\left(\lambda_{c} \mu_{c}\right) \times(14,0)\right](\lambda \mu) \mid(\lambda \mu) i\right\rangle \\
& \times\left\langle\left[\left[\phi\left({ }^{12} \mathrm{C}\right)^{(04)} \times \phi\left({ }^{12} \mathrm{C}\right)^{(04)}\right]^{\left(\lambda_{c} \mu_{c}\right)} \times \chi(\overline{\mathbf{R}})^{(14,0)}\right]_{\alpha}^{(\lambda \mu)}\right| \\
& \times \mathscr{A}\left|\left[\left[\phi_{f^{\prime}}^{(00)} \times \phi_{A}^{\left(\lambda_{c}^{\prime} \mu^{\prime} f^{\prime}\right.}\right]^{\left(\lambda_{c}^{\prime} \mu_{c}^{\prime}\right)} \times \chi(\boldsymbol{R})^{(14-\Delta, 0)}\right]_{\alpha}^{\left(\lambda_{\mu}\right)}\right\rangle \equiv\left(f^{\prime}+A-f^{\prime}\left(\lambda_{c}^{\prime} \mu_{c}^{\prime}\right) \mid(\lambda \mu) i\right) .
\end{aligned}
$$

The factors $\left(f^{\prime}+A-f^{\prime}\left(\lambda_{\mathrm{c}}^{\prime} \mu_{\mathrm{c}}^{\prime}\right)((\lambda \mu) i)\right.$, defined by eq. (123), are shown in table 8 . The combinations of the numbers of tables 7 and 8 show that the state $(10,4) 1$ can be expected to make the dominant contribution to the product of partial width amplitudes 
TABLE 8

The factors $\left(f^{\prime}+A-f^{\prime}\left(\lambda^{\prime} \mu_{\mathrm{c}}^{\prime}\right) \mid(\lambda \mu) i\right)$

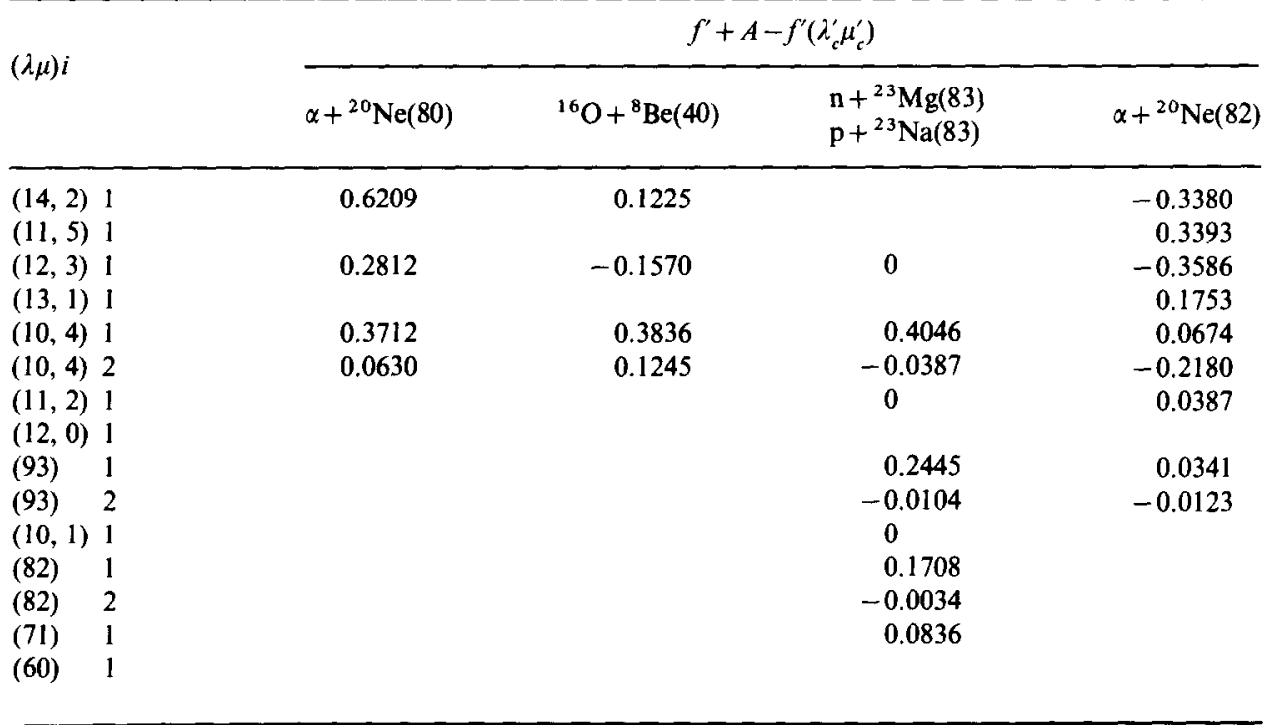

$\Gamma_{v c}^{\frac{1}{2}} \Gamma_{v c^{\prime}}^{\frac{1}{2}}$ for most of the observed channels $c^{\prime}$. However, the states $(14,2) 1$ and $(11,5) 1$ can make significant contributions to the $\alpha+{ }^{20} \mathrm{Ne}$ channels, and the states (93)1 and (82)1 may be important for the ${ }^{23} \mathrm{Na}+\mathrm{p}$ and ${ }^{23} \mathrm{Mg}+\mathrm{n}$ channels.

[It should be pointed out that the spectroscopic amplitudes of tables 7 and 8 and eqs. (120)-(122) are conventional spectroscopic amplitudes, using the language of a recent series of papers by Fliessbach ${ }^{56}$ ). The so-called new amplitudes would be renormalized with factors $\Lambda_{c^{\prime}}^{-\frac{1}{2}}$ for the exit channels. With these factors the entries of table 8 for the ${ }^{23} \mathrm{Na}+\mathrm{p}$ channels are changed by at most $1 \%$; while the entries for the $\alpha+{ }^{20} \mathrm{Ne}(80)$ channels, e.g., would change from $0.6209,0.2812,0.3712,0.0630$, to 1 , $0.5907,0.4958,0.0842$, respectively. Such a renormalization thereforc leads to no essential change in the basic conclusions about the nature of the dominant components $(\lambda \mu) i$ in the various exit channels.]

\section{Concluding remarks}

Further developments have been made in a method which reduces the calculation of the complicated multi-dimensional integrals for norm and overlap matrix elements in a cluster-model basis to purely algebraic techniques involving the algebra of SU(3) recoupling transformations. The method involves the calculation of the BargmannSegal integral transform of the antisymmetrization operator and the expansion of this transform in terms of appropriate $\mathrm{SU}(3)$ coupled Bargmann space functions. A new variant of this method makes it possible to calculate norm and overlap matrix elements for cluster systems made up of two heavy fragments other than closed-shell 
nuclei. In this method, BS transforms are first calculated for $n$-particle subsystems of spatial symmetry characterized by single-columned Young tableaux. The BS transforms for the full $A$-particle system are then built from suitably symmetrized combinations of these single-column transforms. Both in the construction of the single-column transforms and in their combination, $S U(3)$ recoupling transformations are used to advantage.

The example of the ${ }^{12} \mathrm{C}+{ }^{12} \mathrm{C}$ system illustrates the feasibility of this technique. Spectroscopic amplitudes have been calculated connecting the normalized, fully antisymmetrized ${ }^{12} \mathrm{C}+{ }^{12} \mathrm{C}$ molecular basis states to exit channels with various fragment decompositions of the $A=24$ system. These amplitudes may serve as a diagnostic tool in an attempt to gain an understanding of the microscopic structure of the underlying quasi-bound states. The details of this attempt are left to a future study.

The techniques used here in the calculation of overlap kernels can easily be generalized to include the overlap of an $f+(A-f)$ particle cluster function with an $(A-2)+2$ particle fragment system, with arbitrary 2-particle excitations, and thereby lead directly to a calculation of interaction kernels, using similar techniques.

One of the authors (K.T.H.) gratefully acknowledges the support of the Alexander von Humboldt Foundation through its US Senior Scientist program and extends his sincere thanks to Prof. H. A. Weidenmüller for the hospitality of the Max-PlanckInstitut für Kernphysik, Heidelberg. One of us (W.Z.) extends his thanks to Dr. H. M. Hofmann for valuable discussions and acknowledges financial support from the Universidad Autonoma de Mexico during his stays in Mexico and from the MaxPlanck-Institut für Kernphysik during his stay in Heidelberg.

\section{Appendix A}

SU(3) RECOUPLING COEFFICIENTS; A COLLECTION OF USEFUL SPECIAL PROPERTIES

Many special properties of the SU(3) recoupling coefficients have been used throughout the text. These are collected here for easy reference. (Derivations follow simply from properties given in ref. ${ }^{36,54,57-59}$ ).

\section{A.1. SU(3) RACAH COEFFICIENTS}

A.1.1. Definitions. The SU(3) Racah or 6- $(\lambda \mu)$ coefficients in unitary form, $(U$-coefficients), are defined by the recoupling transformation

$$
\begin{aligned}
& \left.\mathbb{I}\left[\left[\left(\lambda_{1} \mu_{1}\right)\left(\lambda_{2} \mu_{2}\right)\right]\left(\lambda_{12} \mu_{12}\right) \rho_{12}\left(\lambda_{3} \mu_{3}\right)\right](\lambda \mu) \rho_{12,3} \ldots\right\rangle \\
& \quad=\sum_{\left(\lambda_{23} \mu_{23}\right) \rho_{23} \rho_{1,23}}\left|\left[\left(\lambda_{1} \mu_{1}\right)\left[\left(\lambda_{2} \mu_{2}\right)\left(\lambda_{3} \mu_{3}\right)\right]\left(\lambda_{23} \mu_{23}\right) \rho_{23}\right](\lambda \mu) \rho_{1,23} \ldots\right\rangle \\
& \quad \times U\left(\left(\lambda_{1} \mu_{1}\right)\left(\lambda_{2} \mu_{2}\right)(\lambda \mu)\left(\lambda_{3} \mu_{3}\right) ;\left(\lambda_{12} \mu_{12}\right) \rho_{12} \rho_{12,3} ;\left(\lambda_{23} \mu_{23}\right) \rho_{23} \rho_{1,23}\right),
\end{aligned}
$$


where ... stands for a convenient set of subgroup labels for the irreducible representation $(\lambda \mu)$. Alternately, making use of the unitary property and reality of the transformation coefficients,

$$
\begin{aligned}
& \left|\left[\left(\lambda_{1} \mu_{1}\right)\left[\left(\lambda_{2} \mu_{2}\right)\left(\lambda_{3} \mu_{3}\right)\right]\left(\lambda_{23} \mu_{23}\right) \rho_{23}\right](\lambda \mu) \rho_{1,23} \ldots\right\rangle \\
& =\sum_{\left(\lambda_{12 \mu_{1}}\right) \rho_{12 \rho_{12,3}}}\left|\left[\left[\left(\lambda_{1} \mu_{1}\right)\left(\lambda_{2} \mu_{2}\right)\right]\left(\lambda_{12} \mu_{12}\right) \rho_{12}\left(\lambda_{3} \mu_{3}\right)\right](\lambda \mu) \rho_{12,3} \ldots\right\rangle \\
& \quad \times U\left(\left(\lambda_{1} \mu_{1}\right)\left(\lambda_{2} \mu_{2}\right)(\lambda \mu)\left(\lambda_{3} \mu_{3}\right) ;\left(\lambda_{12} \mu_{12}\right) \rho_{12} \rho_{12,3} ;\left(\lambda_{23} \mu_{23}\right) \rho_{23} \rho_{1,23}\right) .
\end{aligned}
$$

The notation is a straightforward generalization of that introduced by Racah. The outer multiplicity labels $\rho_{12}, \rho_{12,3}, \rho_{23}$ and $\rho_{1,23}$ are needed to distinguish multiple occurences of a given representation in the Kronecker products of the recoupling transformation. E.g., if a specific $(\lambda \mu)$ occurs in the product $\left(\lambda_{1} \mu_{1}\right) \times\left(\lambda_{23} \mu_{23}\right)$ with a $d$-fold multiplicity $(d>1)$, then the label $\rho_{1,23}$ is needed and has the values $\rho_{1,23}=$ $1,2, \ldots, d=\rho_{1,23_{\max }}$. The conventions of Draayer and Akiyama ${ }^{36}$ ), based on the upper Gel'fand pattern coupling scheme of Biedenharn and Louck ${ }^{60}$ ), are to be used throughout. In this coupling scheme simple symmetry properties survive under conjugation, $\left(\lambda_{i} \mu_{i}\right) \rightarrow\left(\mu_{i} \lambda_{i}\right) i=1,2,3$ in the coupling $\left(\lambda_{1} \mu_{1}\right) \times\left(\lambda_{2} \mu_{2}\right) \rightarrow\left(\lambda_{3} \mu_{3}\right)$, as well as under the interchange $\left(\lambda_{1} \mu_{1}\right) \leftrightarrow\left(\lambda_{3} \mu_{3}\right),\left(\lambda_{2} \mu_{2}\right) \rightarrow\left(\mu_{2} \lambda_{2}\right)$. Interchanges $\left(\lambda_{1} \mu_{1}\right) \leftrightarrow$ $\left(\lambda_{2} \mu_{2}\right)$, however, are not simple. In the general case

$$
\left|\left[\left(\lambda_{1} \mu_{1}\right)\left(\lambda_{2} \mu_{2}\right)\right]\left(\lambda_{3} \mu_{3}\right) \rho \ldots\right\rangle=\sum_{\rho^{\prime}} M_{\rho \rho^{\prime}}\left|\left[\left(\lambda_{2} \mu_{2}\right)\left(\lambda_{1} \mu_{1}\right)\right]\left(\lambda_{3} \mu_{3}\right) \rho^{\prime} \ldots\right\rangle,
$$

where $M_{p p^{\prime}}$ is a $d \times d$ unitary (real) matrix. In the case of a multiplicity-free coupling, however with $\rho_{\max }=1$ :

$$
M_{11}=(-1)^{\lambda_{1}+\mu_{1}+\lambda_{2}+\mu_{2}+\lambda_{3}+\mu_{3}} .
$$

In most of the recoupling transformations used in this work the SU(3) couplings are free of outer multiplicity, so that all (or many) of the $\rho$-labels are unnecessary. In such cases the $\rho$-labels are replaced by a dash or are simply omitted altogether. E.g., the following multiplicity-free recoupling coefficient is denoted by

$$
U\left(\left(\lambda_{1} 0\right)\left(\lambda_{2} \mu_{2}\right)(\lambda \mu)\left(\lambda_{3} 0\right) ;\left(\lambda_{12} \mu_{12}\right)_{--} ;\left(\lambda_{23} \mu_{23}\right)_{--}\right)
$$

or simply by

$$
U\left(\left(\lambda_{1} 0\right)\left(\lambda_{2} \mu_{2}\right)(\lambda \mu)\left(\lambda_{3} 0\right) ;\left(\lambda_{12} \mu_{12}\right)\left(\lambda_{23} \mu_{23}\right)\right) \text {. }
$$

A.1.2. Special cases. Whenever the recoupling transformation is $1 \times 1$ the $U$-coefficient has the value +1 ; e.g. in recoupling transformation in which $\left(\lambda_{1} \mu_{1}\right)$ or $\left(\lambda_{2} \mu_{2}\right)$ or $\left(\lambda_{3} \mu_{3}\right)$ or $(\lambda \mu)=(00)$. Also,

$$
\begin{aligned}
U\left(\left(\lambda_{1} \mu_{1}\right)\left(\lambda_{2} \mu_{2}\right)\left(\lambda_{1} \mu_{1}\right)\left(\mu_{2} \lambda_{2}\right) ;\left(\lambda_{12} \mu_{12}\right) \rho \rho^{\prime} ;(00)_{--}\right) & \\
= & (-1)^{\lambda_{1}+\mu_{1}+\lambda_{2}+\mu_{2}+\lambda_{12}+\mu_{12}} \delta_{\rho \rho^{\prime}}\left[\frac{d\left(\lambda_{12} \mu_{12}\right)}{d\left(\lambda_{1} \mu_{1}\right) d\left(\lambda_{2} \mu_{2}\right)}\right]^{\frac{1}{2}},
\end{aligned}
$$


where the dimension of the irreducible representation $(\lambda \mu)$ is given by

$$
d(\lambda \mu)=\frac{1}{2}(\lambda+1)(\mu+1)(\lambda+\mu+2)
$$

But

$U\left(\left(\lambda_{1} \mu_{1}\right)\left(\mu_{1} \lambda_{1}\right)\left(\lambda_{3} \mu_{3}\right)\left(\lambda_{3} \mu_{3}\right) ;(00)_{-} ;\left(\lambda_{23} \mu_{23}\right) \rho \rho^{\prime}\right)$

$$
=(-1)^{\lambda_{1}+\mu_{1}+\lambda_{3}+\mu_{3}+\lambda_{23}+\mu_{23}} C_{p p^{\prime}}\left[\frac{\mathrm{d}\left(\lambda_{23} \mu_{23}\right)}{d\left(\lambda_{1} \mu_{1}\right) d\left(\lambda_{3} \mu_{3}\right)}\right]^{\frac{1}{2}} .
$$

In the general case, $C_{\rho \rho^{\prime}}$ is a unitary matrix (and not the unit matrix). In the special case when all couplings are free of multiplicity, $C_{\rho \rho^{\prime}}\left(\equiv C_{11}\right)=+1$.

A.1.3. Symmetry property under conjugation.

$$
\begin{aligned}
& U\left(\left(\lambda_{1} \mu_{1}\right)\left(\lambda_{2} \mu_{2}\right)(\lambda \mu)\left(\lambda_{3} \mu_{3}\right) ;\left(\lambda_{12} \mu_{12}\right) \rho_{12} \rho_{12,3} ;\left(\lambda_{23} \mu_{23}\right) \rho_{23} \rho_{1,23}\right)
\end{aligned}
$$

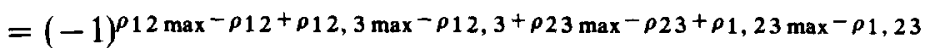

$$
\begin{aligned}
& \times U\left(\left(\mu_{1} \lambda_{1}\right)\left(\mu_{2} \lambda_{2}\right)(\mu \lambda)\left(\mu_{3} \lambda_{3}\right) ;\left(\mu_{12} \lambda_{12}\right) \rho_{12} \rho_{12,3} ;\left(\mu_{23} \lambda_{23}\right) \rho_{23} \rho_{1,23}\right) .
\end{aligned}
$$

A.1.4. Special symmetry properties. For the completely multiplicity-free case

$$
\begin{aligned}
U\left(\left(\lambda_{1} \mu_{1}\right)\left(\lambda_{2} \mu_{2}\right)(\lambda \mu)\left(\lambda_{3} \mu_{3}\right) ;\left(\lambda_{12} \mu_{12}\right)_{--} ;\left(\lambda_{23} \mu_{23}\right)_{--}\right) \\
=U\left(\left(\lambda_{3} \mu_{3}\right)\left(\lambda_{2} \mu_{2}\right)(\lambda \mu)\left(\lambda_{1} \mu_{1}\right) ;\left(\lambda_{23} \mu_{23}\right)_{--} ;\left(\lambda_{12} \mu_{12}\right)_{--}\right) \\
=U\left(\left(\lambda_{2} \mu_{2}\right)\left(\lambda_{1} \mu_{1}\right)\left(\mu_{3} \lambda_{3}\right)(\mu \lambda) ;\left(\lambda_{12} \mu_{12}\right)_{--} ;\left(\mu_{23} \lambda_{23}\right)_{--}\right) .
\end{aligned}
$$

For the special case, with $\rho_{23_{\max }}=1$,

$$
\begin{aligned}
U\left(\left(\lambda_{1} \mu_{1}\right)\left(\lambda_{2} \mu_{2}\right)(\lambda \mu)\left(\lambda_{3} \mu_{3}\right) ;\left(\lambda_{12} \mu_{12}\right) \rho_{12} \rho_{12,3} ;\left(\lambda_{23} \mu_{23}\right)_{-} \rho_{1,23}\right) \\
=(-1)^{\lambda_{1}+\mu_{1}+\lambda_{3}+\mu_{3}+\lambda_{12}+\mu_{12}+\lambda_{23}+\mu_{23}}\left[\frac{d\left(\lambda_{12} \mu_{12}\right) d\left(\lambda_{23} \mu_{23}\right)}{d\left(\lambda_{1} \mu_{1}\right) d\left(\lambda_{3} \mu_{3}\right)}\right]^{t} \\
\times U\left(\left(\lambda_{12} \mu_{12}\right)\left(\mu_{2} \lambda_{2}\right)(\lambda \mu)\left(\lambda_{23} \mu_{23}\right) ;\left(\lambda_{1} \mu_{1}\right) \rho_{12} \rho_{1,23} ;\left(\lambda_{3} \mu_{3}\right)_{-} \rho_{12,3}\right) .
\end{aligned}
$$

A.1.5. Equivalence with $S U(2)$ coefficients. If all the U(3) [rather than $\mathrm{SU}(3)$ ] irreducible representations in the recoupling coefficient can be characterized by at most 2-rowed Young tableaux, the $S U(3) U$-coefficient is equivalent to an $S U(2)$ coefficient; that is, if

then

$$
\lambda+2 \mu=\lambda_{1}+2 \mu_{1}+\lambda_{2}+2 \mu_{2}+\lambda_{3}+2 \mu_{3},
$$

$U\left(\left(\lambda_{1} \mu_{1}\right)\left(\lambda_{2} \mu_{2}\right)(\lambda \mu)\left(\lambda_{3} \mu_{3}\right) ;\left(\lambda_{12} \mu_{12}\right)\left(\lambda_{23} \mu_{23}\right)\right)$

$$
\begin{aligned}
& =U_{\mathrm{SU}(2)}\left(\frac{\lambda_{1}}{2} \frac{\lambda_{2}}{2} \frac{\lambda}{2} \frac{\lambda_{3}}{2} ; \frac{\lambda_{12}}{2} \frac{\lambda_{23}}{2}\right) \\
& =(-1)^{\frac{1}{3}\left(\lambda_{1}+\lambda_{2}+\lambda_{3}+\lambda\right)}\left[\left(\lambda_{12}+1\right)\left(\lambda_{23}+1\right)\right]^{\frac{1}{2}}\left\{\begin{array}{lll}
\frac{1}{2} \lambda_{1} & \frac{1}{2} \lambda_{2} & \frac{1}{2} \lambda_{12} \\
\frac{1}{2} \lambda_{3} & \frac{1}{2} \lambda & \frac{1}{2} \lambda_{23}
\end{array}\right\},
\end{aligned}
$$


where the symbol in curly brackets is a conventional angular momentum $6-j$ coefficient.

A.1.6. Special values for maximal couplings. A $U$-coefficient with two stretched couplings of 1-rowed representations has the simple value

$U((a 0)(b 0)(\lambda \mu)(c 0) ;(a+b, 0)(b+c, 0))=\left[\frac{a ! c !(b+c-\mu) !(a+b-\mu) !}{(b+a) !(b+c) !(c-\mu) !(a-\mu) !}\right]^{\frac{1}{2}}$

with

$$
\lambda+2 \mu=a+b+c .
$$

A $U$-coefficient with one stretched 1-rowed coupling and a maximal coupling of the type $(0 a) \times(b 0) \rightarrow(0, a-b)$ has the value

$U((0 a)(b 0)(\lambda \mu)(c 0) ;(0, a-b)(b+c, 0))$

$$
=\left[\frac{d(0, a-b)}{d(0 a)}\right]^{\frac{1}{2}}\left[\frac{(a-b) ! c !(a-\mu) !(\lambda+a+2) !}{a !(b+c) !(a-b-\mu) !(\lambda+a-b+2) !}\right]^{\frac{1}{2}} .
$$

Also,

$$
\begin{aligned}
& U((a-b, 0)(b 0)(\mu \lambda)(0, b+c) ;(a 0)(0 c)) \\
& =\left[\frac{d(c 0)}{d(b+c, 0)}\right]^{\frac{1}{2}}\left[\frac{(a-b) ! c !(a-\mu) !(\lambda+a+2) !}{a !(b+c) !(a-b-\mu) !(\lambda+a-b+2) !}\right]^{\frac{1}{2}} .
\end{aligned}
$$

\section{A.2. $\operatorname{SU}(3) 9-(\lambda \mu)$ COEFFICIENTS}

A.2.1. Defining equation. The $9-(\lambda \mu)$ recoupling coefficient, in unitary form $\left.{ }^{61}\right)$, is defined by the recoupling transformation

$$
\begin{aligned}
\mathbb{I} & {\left.\left.\left[\left(\lambda_{1} \mu_{1}\right)\left(\lambda_{2} \mu_{2}\right)\right]\left(\lambda_{12} \mu_{12}\right) \rho_{12}\left[\left(\lambda_{3} \mu_{3}\right)\left(\lambda_{4} \mu_{4}\right)\right]\left(\lambda_{34} \mu_{34}\right) \rho_{34}\right](\lambda \mu) \rho_{12,34} \ldots\right\rangle } \\
& =\sum_{\substack{\left(\lambda_{13} \mu_{13}\right)\left(\lambda_{24} \mu_{24}\right) \\
\rho_{132} \rho_{24} \rho_{13}, 24}}\left|\left[\left[\left(\lambda_{1} \mu_{1}\right)\left(\lambda_{3} \mu_{3}\right)\right]\left(\lambda_{13} \mu_{13}\right) \rho_{13}\left[\left(\lambda_{2} \mu_{2}\right)\left(\lambda_{4} \mu_{4}\right)\right]\left(\lambda_{24} \mu_{24}\right) \rho_{24}\right](\lambda \mu) \rho_{13,24} \ldots\right\rangle \\
& \times\left[\begin{array}{llll}
\left(\lambda_{1} \mu_{1}\right) & \left(\lambda_{2} \mu_{2}\right) & \left(\lambda_{12} \mu_{12}\right) & \rho_{12} \\
\left(\lambda_{3} \mu_{3}\right) & \left(\lambda_{4} \mu_{4}\right) & \left(\lambda_{34} \mu_{34}\right) & \rho_{34} \\
\left(\lambda_{13} \mu_{13}\right) & \left(\lambda_{24} \mu_{24}\right) & (\lambda \mu) & \rho_{13,24} \\
\rho_{13} & \rho_{24} & \rho_{12,34}
\end{array}\right] .
\end{aligned}
$$

A.2.2. Special cases. Special 9-( $\lambda \mu$ ) coefficients with one or several $(00)$ representations are frequently needed in this work.

$$
\left[\begin{array}{llll}
\left(\lambda_{1} \mu_{1}\right) & \left(\mu_{1} \lambda_{1}\right) & (00) & - \\
\left(\lambda_{3} \mu_{3}\right) & \left(\mu_{3} \lambda_{3}\right) & (00) & - \\
(\lambda \mu) & (\mu \lambda) & (00) & - \\
\rho & \rho^{\prime} & -
\end{array}\right]=\delta_{\rho \rho^{\prime}}(-1)^{\rho_{\max }-\rho}\left[\frac{d(\lambda \mu)}{d\left(\lambda_{1} \mu_{1}\right) d\left(\lambda_{3} \mu_{3}\right)}\right]^{\frac{1}{2}} .
$$


Also, with $\rho_{13_{\max }}=1$,

$$
\begin{gathered}
{\left[\begin{array}{llll}
\left(\lambda_{1} \mu_{1}\right) & \left(\mu_{1} \lambda_{1}\right) & (00) & - \\
\left(\lambda_{3} \mu_{3}\right) & \left(\lambda_{4} \mu_{4}\right) & (\lambda \mu) & \rho_{34} \\
\left(\lambda_{13} \mu_{13}\right) & \left(\lambda_{24} \mu_{24}\right) & (\lambda \mu) & \rho \\
- & \rho_{24} & -
\end{array}\right]=\left[\frac{d\left(\lambda_{13} \mu_{13}\right)}{d\left(\lambda_{1} \mu_{1}\right) d\left(\lambda_{3} \mu_{3}\right)}\right]^{\frac{1}{2}}} \\
\quad \times U\left(\left(\lambda_{13} \mu_{13}\right)\left(\mu_{1} \lambda_{1}\right)(\lambda \mu)\left(\lambda_{4} \mu_{4}\right) ;\left(\lambda_{3} \mu_{3}\right)_{-} \rho_{34} ;\left(\lambda_{24} \mu_{24}\right) \rho_{24} \rho\right),
\end{gathered}
$$

while, with $\rho_{24_{\max }}=1$,

$$
\begin{aligned}
& \left.\begin{array}{llll}
\left(\lambda_{1} \mu_{1}\right) & \left(\lambda_{2} \mu_{2}\right) & (\lambda \mu) & \rho_{12} \\
\left(\mu_{4} \lambda_{4}\right) & \left(\lambda_{4} \mu_{4}\right) & (00) & - \\
\left(\lambda_{13} \mu_{13}\right) & \left(\lambda_{24} \mu_{24}\right) & (\lambda \mu) & \rho \\
\rho_{13} & - & -
\end{array}\right]=\left[\frac{d\left(\lambda_{24} \mu_{24}\right)}{d\left(\lambda_{2} \mu_{2}\right) d\left(\lambda_{4} \mu_{4}\right)}\right]^{\frac{1}{2}} \\
& \quad \times U\left(\left(\lambda_{1} \mu_{1}\right)\left(\mu_{4} \lambda_{4}\right)(\lambda \mu)\left(\lambda_{24} \mu_{24}\right) ;\left(\lambda_{13} \mu_{13}\right) \rho_{13} \rho ;\left(\lambda_{2} \mu_{2}\right)_{-} \rho_{12}\right) .
\end{aligned}
$$

A.2.3. Symmetry property under conjugation.

$$
\begin{array}{r}
{\left[\begin{array}{llll}
\left(\lambda_{1} \mu_{1}\right) & \left(\lambda_{2} \mu_{2}\right) & \left(\lambda_{12} \mu_{12}\right) & \rho_{12} \\
\left(\lambda_{3} \mu_{3}\right) & \left(\lambda_{4} \mu_{4}\right) & \left(\lambda_{34} \mu_{34}\right) & \rho_{34} \\
\left(\lambda_{13} \mu_{13}\right) & \left(\lambda_{24} \mu_{24}\right) & (\lambda \mu) & \rho_{13,24} \\
\rho_{13} & \rho_{24} & \rho_{12,34} &
\end{array}\right]} \\
=(-1)^{\Sigma_{s}\left(\rho_{5 \max }-\rho_{s}\right)}\left[\begin{array}{llll}
\left(\mu_{1} \lambda_{1}\right) & \left(\mu_{2} \lambda_{2}\right) & \left(\mu_{12} \lambda_{12}\right) & \rho_{12} \\
\left(\mu_{3} \lambda_{3}\right) & \left(\mu_{4} \lambda_{4}\right) & \left(\mu_{34} \lambda_{34}\right) & \rho_{34} \\
\left(\mu_{13} \lambda_{13}\right) & \left(\mu_{24} \lambda_{24}\right) & (\mu \lambda) & \rho_{13,24} \\
\rho_{13} & \rho_{24} & \rho_{12,34}
\end{array}\right],
\end{array}
$$

where the sum over $s$ in the phase factor sums over all six multiplicity labels.

A.2.4. Special symmetry property.

$$
\begin{aligned}
& {\left[\begin{array}{llll}
\left(\lambda_{1} \mu_{1}\right) & \left(\lambda_{2} \mu_{2}\right) & \left(\lambda_{12} \mu_{12}\right) & \rho_{12} \\
\left(\lambda_{3} \mu_{3}\right) & \left(\lambda_{4} \mu_{4}\right) & \left(\lambda_{34} \mu_{34}\right) & \rho_{34} \\
\left(\lambda_{13} \mu_{13}\right) & \left(\lambda_{24} \mu_{24}\right) & (\lambda \mu) & \rho_{13,24} \\
\rho_{13} & \rho_{24} & \rho_{12,34}
\end{array}\right]} \\
& \quad=(-1)^{\Sigma_{i}\left(\lambda_{i}+\mu_{i j}\right)}(-1)^{\rho_{24} \max -\rho_{24}}\left[\frac{d\left(\lambda_{12} \mu_{12}\right) d\left(\lambda_{34} \mu_{34}\right) d\left(\lambda_{13} \mu_{13}\right)}{d\left(\lambda_{1} \mu_{1}\right) d\left(\lambda_{3} \mu_{3}\right) d(\lambda \mu)}\right]^{\frac{1}{2}}
\end{aligned}
$$




$$
\times\left[\begin{array}{llll}
\left(\lambda_{12} \mu_{12}\right) & \left(\mu_{2} \lambda_{2}\right) & \left(\lambda_{1} \mu_{1}\right) & \rho_{12} \\
\left(\lambda_{34} \mu_{34}\right) & \left(\mu_{4} \lambda_{4}\right) & \left(\lambda_{3} \mu_{3}\right) & \rho_{34} \\
(\lambda \mu) & \left(\mu_{24} \lambda_{24}\right) & \left(\lambda_{13} \mu_{13}\right) & \rho_{13.24} \\
\rho_{12,34} & \rho_{24} & \rho_{13} &
\end{array}\right]
$$

where the sum over $\lambda_{i}+\mu_{i}$ factors is a sum over all nine representations.

A.2.5. Equivalence with $S U(2)$ coefficients. If all $\mathrm{U}(3)$ [rather than $\mathrm{SU}(3)]$ irreducible representations in the coupling coefficient can be characterized by at most 2-rowed tableaux, the 9- $(\lambda \mu)$ coefficient is equivalent to an SU(2) 9-j coefficient; that is, if

then

$$
\lambda+2 \mu=\lambda_{1}+2 \mu_{1}+\lambda_{2}+2 \mu_{2}+\lambda_{3}+2 \mu_{3}+\lambda_{4}+2 \mu_{4},
$$

$$
\begin{aligned}
& {\left[\begin{array}{lll}
\left(\lambda_{1} \mu_{1}\right) & \left(\lambda_{2} \mu_{2}\right) & \left(\lambda_{12} \mu_{12}\right) \\
\left(\lambda_{3} \mu_{3}\right) & \left(\lambda_{4} \mu_{4}\right) & \left(\lambda_{34} \mu_{34}\right) \\
\left(\lambda_{13} \mu_{13}\right) & \left(\lambda_{24} \mu_{24}\right) & (\lambda \mu)
\end{array}\right]=\left[\left(\lambda_{12}+1\right)\left(\lambda_{34}+1\right)\left(\lambda_{13}+1\right)\left(\lambda_{24}+1\right)\right]^{\frac{1}{2}} } \\
& \times\left\{\begin{array}{lll}
\frac{1}{2} \lambda_{1} & \frac{1}{2} \lambda_{2} & \frac{1}{2} \lambda_{12} \\
\frac{1}{2} \lambda_{3} & \frac{1}{2} \lambda_{4} & \frac{1}{2} \lambda_{34} \\
\frac{1}{2} \lambda_{13} & \frac{1}{2} \lambda_{24} & \frac{1}{2} \lambda
\end{array}\right\},
\end{aligned}
$$

where the coefficient in curly brackets is a conventional angular momentum 9-j coefficient. Note that such a $9-(\lambda \mu)$ coefficient is completely free of multiplicities, and all $\rho$-labels are simply omitted.

A.2.6. Special values for maximal couplings. A 9- $(\lambda \mu)$ coefficient with four stretched couplings of 1-rowed representations can be expressed in terms of one simple sum:

$$
\begin{aligned}
& {\left[\begin{array}{lll}
\left(\lambda_{1} 0\right) & \left(\lambda_{2} 0\right) & \left(\lambda_{1}+\lambda_{2}, 0\right) \\
\left(\lambda_{3} 0\right) & \left(\lambda_{4} 0\right) & \left(\lambda_{3}+\lambda_{4}, 0\right) \\
\left(\lambda_{1}+\lambda_{3}, 0\right) & \left(\lambda_{2}+\lambda_{4}, 0\right) & (\lambda \mu)
\end{array}\right] } \\
&=\lambda_{1} ! \lambda_{2} ! \lambda_{3} ! \lambda_{4} !\left[\frac{\left(\lambda_{1}+\lambda_{2}-\mu\right) !\left(\lambda_{3}+\lambda_{4}-\mu\right) !\left(\lambda_{1}+\lambda_{3}-\mu\right) !\left(\lambda_{2}+\lambda_{4}-\mu\right) !}{\left(\lambda_{1}+\lambda_{2}\right) !\left(\lambda_{3}+\lambda_{4}\right) !\left(\lambda_{1}+\lambda_{3}\right) !\left(\lambda_{2}+\lambda_{4}\right) !}\right]^{\frac{1}{2}} \\
& \times \sum_{x} \frac{(-1)^{x} \mu !}{x !(\mu-x) !} \frac{1}{\left(\lambda_{2}-x\right) !\left(\lambda_{3}-x\right) !\left(\lambda_{1}+x-\mu\right) !\left(\lambda_{4}+x-\mu\right) !}
\end{aligned}
$$

with

$$
\lambda+2 \mu=\lambda_{1}+\lambda_{2}+\lambda_{3}+\lambda_{4}
$$




\section{Appendix B}

PROPERTIES OF SU(3) COUPLED BARGMANN SPACE FUNCTIONS

Manipulations of SU(3) coupled $K$-space functions which are needed to gain the basic canonical form of the Bargmann-space transforms are facilitated by several subsidiary formulae. The more important of these are collected here for easy reference. (Some sample derivations are given in subsect. B.4.)

\section{B.1. BASIC BUILDING BLOCKS}

The SU(3) coupling of Bargmann space functions in the same variable $K$ requires a renormalization factor. This follows at once from the norm of the $K$-space oscillator function.

$$
\left.\left[P(K)^{\left(Q_{1} 0\right)}\right) \times P(K)^{\left(Q_{2} 0\right)}\right]_{\alpha}^{(\lambda \mu)}=\delta_{(\lambda \mu)\left(Q_{1}+Q_{2}, 0\right)}\left[\frac{\left(Q_{1}+Q_{2}\right) !}{Q_{1} ! Q_{2} !}\right]^{t} P(K)_{\alpha}^{\left(Q_{1}+Q_{2}, 0\right)},
$$

where $\alpha$ is any convenient subgroup label set; (e.g., $\alpha=\kappa L M$ or $\varepsilon \Lambda M_{A}$ ).

The basic building block for the expansion of $K$-space functions is the scalar product

$$
\left(K_{i} \cdot K_{j}^{*}\right)=\sqrt{3}\left[P\left(K_{i}\right)^{(10)} \times P\left(K_{j}^{*}\right)^{(01)}\right]_{00}^{(00)} .
$$

This leads to

$$
\left(\boldsymbol{K}_{i} \cdot \boldsymbol{K}_{j}^{*}\right)^{n}=n ![d(n, 0)]^{\frac{1}{2}}\left[P\left(\boldsymbol{K}_{i}\right)^{(n 0)} \times P\left(K_{j}^{*}\right)^{(0 n)}\right]_{00}^{(00)} .
$$

[The subgroup label 00 can be interpreted as $L=M=0$ or as $\varepsilon=M_{A}(=A)=0$.]

The vector product of two $K$-space vectors, ((10)-tensors), is a $K$-space pseudovector, [(01)-tensor],

$$
\left[K_{1} \times K_{2}\right]_{\alpha}=\sqrt{2}\left[P\left(K_{1}\right)^{(01)} \times P\left(K_{2}\right)^{(10)}\right]_{\alpha}^{(01)} .
$$

This leads to

$$
P\left(\left[K_{1} \times K_{2}\right]_{\alpha}^{(0 n)}=\sqrt{(n+1) !}\left[P\left(K_{1}\right)^{(n 0)} \times P\left(K_{2}\right)^{(n 0)}\right]_{\alpha}^{(0 n)} .\right.
$$

\section{B.2. COMBINATIONS OF SCALAR PRODUCTS}

The combination of two scalar products with one common $K$-value can be written in suitably SU(3) coupled form by the use of relations (B.3), (A.16) and (B.1)

$$
\begin{aligned}
\left(K_{1} \cdot K_{2}^{*}\right)^{a}\left(K_{1} \cdot K_{3}^{*}\right)^{b}=[a ! b !(a+b) ! d(a+b, 0)]^{\frac{1}{2}} \\
\times\left[P\left(K_{1}\right)^{(a+b, 0)} \times\left[P\left(K_{2}^{*}\right)^{(0 a)} \times P\left(K_{3}^{*}\right)^{(0 b)}\right]^{(0, a+b)}\right]_{00}^{(00)} .
\end{aligned}
$$


Examples of more complicated products are

$$
\begin{aligned}
\left(\boldsymbol{K}_{1} \cdot \boldsymbol{K}_{2}^{*}\right)^{a}\left(\boldsymbol{K}_{3} \cdot \boldsymbol{K}_{1}^{*}\right)^{b}\left(\boldsymbol{K}_{1} \cdot \boldsymbol{K}_{1}^{*}\right)^{c}=\sum_{(\lambda \mu)} a ! b !\left[\frac{(a+c-\mu) !(b+c-\mu) !}{(a-\mu) !(b-\mu) !}\right]^{\frac{1}{2}}[d(\lambda \mu)]^{\frac{1}{2}} \\
\times\left[\left[P\left(\boldsymbol{K}_{1}\right)^{(a+c, 0)} \times P\left(\boldsymbol{K}_{3}\right)^{(b 0)}\right]^{(\lambda \mu)} \times\left[P\left(\boldsymbol{K}_{2}^{*}\right)^{(0 a)} \times P\left(\boldsymbol{K}_{1}^{*}\right)^{(0 . b+c)}\right]^{(\mu \lambda)}\right]_{00}^{(00)},
\end{aligned}
$$

with $(\lambda \mu)=(a+b+c, 0),(a+b+c-2,1),(a+b+c-4,2), \ldots,(a+c-b, b)$ or $(b-a-c$, $a+c$ ). Similarly,

$$
\begin{aligned}
&\left(\boldsymbol{K}_{1} \cdot\right.\left.\boldsymbol{K}_{1}^{*}\right)^{a}\left(\boldsymbol{K}_{2} \cdot \boldsymbol{K}_{1}^{*}\right)^{b}\left(\boldsymbol{K}_{1} \cdot \boldsymbol{K}_{2}^{*}\right)^{c}\left(\boldsymbol{K}_{2} \cdot \boldsymbol{K}_{2}^{*}\right)^{e} \\
& \quad= \sum_{(\lambda, \mu)}[(a+b) !(a+c) !(b+e) !(c+e) ! d(\lambda \mu)]^{\frac{1}{2}}\left[\begin{array}{lll}
(a 0) & (b 0) & (a+b, 0) \\
(c 0) & (e 0) & (c+e, 0) \\
(a+c, 0) & (b+e, 0) & (\lambda \mu)
\end{array}\right] \\
& \quad \times\left[\left[P\left(K_{1}\right)^{(a+c, 0)} \times P\left(K_{2}\right)^{(b+e, 0)}\right]^{(\lambda \mu)} \times\left[P\left(K_{1}^{*}\right)^{(0, a+b)} \times P\left(K_{2}^{*}\right)^{(0, c+e)}\right]^{(\mu \lambda)}\right]_{00}^{(00)},
\end{aligned}
$$

where the $9-(\lambda \mu)$ coefficient is equivalent to a simple SU(2) coefficient and can by evaluated simply by means of eq. (A.22). If $K$ is a polar vector, [(10)-tensor], while $\boldsymbol{K}_{\mathrm{p}}$ is a pseudovector, ((01)-tensor), e.g. $\boldsymbol{K}_{\mathrm{p}}=\left[\boldsymbol{K}_{1} \times \boldsymbol{K}_{2}\right]$, then

$$
\begin{aligned}
\left(\boldsymbol{K} \cdot \boldsymbol{K}_{\mathrm{p}}\right)^{a}\left(\boldsymbol{K}^{*} \cdot \boldsymbol{K}_{\mathrm{p}}^{*}\right)^{b}\left(\boldsymbol{K}_{\mathrm{p}} \cdot \boldsymbol{K}_{\mathrm{p}}^{*}\right)^{c}\left(\boldsymbol{K} \cdot \boldsymbol{K}^{*}\right)^{e} \\
=\sum_{(\lambda \mu)} c ! e ! \frac{[(a+c-\mu) !(b+c-\mu) !(\lambda+a+c+2) !(\lambda+b+c+2) ! d(\lambda \mu)]^{\frac{1}{2}}}{(c-\mu) !(\lambda+c+2) !} \\
\quad \times\left[\left[P\left(\boldsymbol{K}_{\mathrm{p}}\right)^{(\mathbf{0}, a+c)} \times P(\boldsymbol{K})^{(a+e, 0)}\right]^{(\lambda \mu)} \times\left[P\left(\boldsymbol{K}_{\mathrm{p}}^{*}\right)^{(b+c, 0)} \times P\left(\boldsymbol{K}^{*}\right)^{(0, b+e)}\right]^{(\mu \lambda)}\right]_{00}^{(00)} .
\end{aligned}
$$

\section{B.3. EXPANSIONS OF SPECIAL SU(3)-COUPLED FUNCTIONS}

In many of the manipulations of $K$-space functions it is useful to expand SU(3) coupled functions in terms of scalar products. Particularly useful expansions include $\sqrt{8}\left[\left[P\left(K_{1}\right)^{(10)} \times P\left(K_{1}^{*}\right)^{(01)}\right]^{(11)} \times\left[P(K)^{(10)} \times P\left(K^{*}\right)^{(01)}\right]^{(11)}\right]_{00}^{(00)}$

$$
=\left\{\left(\boldsymbol{K}_{1} \cdot \boldsymbol{K}^{*}\right)\left(\boldsymbol{K} \cdot \boldsymbol{K}_{1}^{*}\right)-\frac{1}{3}\left(\boldsymbol{K}_{1} \cdot \boldsymbol{K}_{1}^{*}\right)\left(\boldsymbol{K} \cdot \boldsymbol{K}^{*}\right)\right\}
$$

$$
\left[P(\boldsymbol{K})^{(20)} \times P\left(K^{*}\right)^{(02)}\right]_{\alpha}^{(11)}=\sqrt{\frac{1}{5}}\left(K \cdot K^{*}\right)\left[P(K)^{(10)} \times P\left(K^{*}\right)^{(01)}\right]_{\alpha}^{(11)},
$$

$\sqrt{27}\left[\left[P\left(K_{1}\right)^{(20)} \times P\left(K_{1}^{*}\right)^{(02)}\right]^{(22)} \times\left[P(K)^{(20)} \times P\left(K^{*}\right)^{(02)}\right]^{(22)}\right]_{00}^{(00)}$

$$
=\left\{\frac{1}{4}\left(K_{1} \cdot K^{*}\right)^{2}\left(K \cdot K_{1}^{*}\right)^{2}-\frac{1}{5}\left(K_{1} \cdot K_{1}^{*}\right)\left(K \cdot K^{*}\right)\left(K_{1} \cdot K^{*}\right)\left(K \cdot K_{1}^{*}\right)+\frac{1}{40}\left(K \cdot K^{*}\right)^{2}\left(K_{1} \cdot K_{1}^{*}\right)^{2}\right\} .
$$

For symmetrically coupled $K$-space vectors, $S U(3)(20)$-tensors, it is useful to use the shorthand notation

$$
P\left(K_{1}, K_{2}\right)_{\alpha}^{(20)} \equiv\left[P\left(K_{1}\right)^{(10)} \times P\left(K_{2}\right)^{(10)}\right]_{\alpha}^{(20)} .
$$


Useful expressions involving such tensors are

$$
\begin{aligned}
& \sqrt{6}\left[\begin{array}{l}
\left.P\left(K_{1}, K_{2}\right)^{(20)} \times P\left(K_{1}^{*}, K_{2}^{*}\right)^{(02)}\right]_{00}^{(00)} \\
\quad=\frac{1}{2}\left\{\left(K_{1} \cdot K_{1}^{*}\right)\left(K_{2} \cdot K_{2}^{*}\right)+\left(K_{1} \cdot K_{2}^{*}\right)\left(K_{2} \cdot K_{1}^{*}\right)\right\}, \\
{\left[P\left(K_{1}, K_{2}\right)^{(20)} \times P\left(K_{1}^{*}, K_{2}^{*}\right)^{(02)}\right]_{a}^{(11)}} \\
\quad=\frac{1}{2} \sqrt{\frac{1}{5}}\left\{\left(K_{1} \cdot K_{2}^{*}\right)\left[P\left(K_{2}\right)^{(10)} \times P\left(K_{1}^{*}\right)^{(01)}\right]_{\alpha}^{(11)}+\left(K_{2} \cdot K_{1}^{*}\right)\left[P\left(K_{1}\right)^{(10)} \times P\left(K_{2}^{*}\right)^{(01)}\right]_{a}^{(11)}\right. \\
\left.\quad+\left(K_{1} \cdot K_{1}^{*}\right)\left[P\left(K_{2}\right)^{(10)} \times P\left(K_{2}^{*}\right)^{(01)}\right]_{a}^{(11)}+\left(K_{2} \cdot K_{2}^{*}\right)\left[P\left(K_{1}\right)^{(10)} \times P\left(K_{1}^{*}\right)^{(01)}\right]_{a}^{(11)}\right\}
\end{array}\right.
\end{aligned}
$$

Also,

$$
\begin{aligned}
\sqrt{27} & {\left[\left[P\left(K_{1}, K_{2}\right)^{(20)} \times P\left(K_{1}^{*}, K_{2}^{*}\right)^{(02)}\right]^{(22)} \times\left[P(K)^{(20)} \times P\left(K^{*}\right)^{(02)}\right]^{(22)}\right]_{00}^{(00)} } \\
& =\left\{\frac{1}{2}\left(\boldsymbol{K} \cdot \boldsymbol{K}_{1}^{*}\right)\left(\boldsymbol{K} \cdot \boldsymbol{K}_{2}^{*}\right)\left(\boldsymbol{K}_{1} \cdot \boldsymbol{K}^{*}\right)\left(\boldsymbol{K}_{2} \cdot \boldsymbol{K}^{*}\right)\right. \\
& +\frac{1}{40}\left(\boldsymbol{K} \cdot \boldsymbol{K}^{*}\right)^{2}\left[\left(\boldsymbol{K}_{1} \cdot \boldsymbol{K}_{1}^{*}\right)\left(\boldsymbol{K}_{2} \cdot \boldsymbol{K}_{2}^{*}\right)+\left(\boldsymbol{K}_{1} \cdot \boldsymbol{K}_{2}^{*}\right)\left(\boldsymbol{K}_{2} \cdot \boldsymbol{K}_{1}^{*}\right)\right] \\
& -\frac{1}{10}\left(\boldsymbol{K} \cdot \boldsymbol{K}^{*}\right)\left[\left(\boldsymbol{K}_{1} \cdot \boldsymbol{K}_{1}^{*}\right)\left(\boldsymbol{K} \cdot \boldsymbol{K}_{2}^{*}\right)\left(\boldsymbol{K}_{2} \cdot \boldsymbol{K}^{*}\right)+\left(\boldsymbol{K}_{2} \cdot \boldsymbol{K}_{2}^{*}\right)\left(\boldsymbol{K} \cdot \boldsymbol{K}_{1}^{*}\right)\left(\boldsymbol{K}_{1} \cdot \boldsymbol{K}^{*}\right)\right. \\
& \left.\left.+\left(\boldsymbol{K}_{1} \cdot \boldsymbol{K}_{2}^{*}\right)\left(\boldsymbol{K} \cdot \boldsymbol{K}_{1}^{*}\right)\left(\boldsymbol{K}_{2} \cdot \boldsymbol{K}^{*}\right)+\left(\boldsymbol{K}_{2} \cdot \boldsymbol{K}_{1}^{*}\right)\left(\boldsymbol{K} \cdot \boldsymbol{K}_{2}^{*}\right)\left(\boldsymbol{K}_{1} \cdot \boldsymbol{K}^{*}\right)\right]\right\} .
\end{aligned}
$$

Similarly,

$$
\begin{gathered}
\sqrt{8}\left[\left[P\left(K_{1}^{*}, K_{2}^{*}\right)^{(02)} \times P\left(K_{3}^{*}\right)^{(01)}\right]^{(11)} \times\left[P(K)^{(10)} \times P\left(K^{*}\right)^{(01)}\right]^{(11)}\right]_{00}^{(00)} \\
=\sqrt{\frac{1}{6}}\left\{\left(\boldsymbol{K} \cdot K_{1}^{*}\right)\left(K^{*} \cdot\left[K_{2}^{*} \times K_{3}^{*}\right]\right)+\left(K \cdot K_{2}^{*}\right)\left(K^{*} \cdot\left[K_{1}^{*} \times K_{3}^{*}\right]\right)\right\} \\
\sqrt{8}\left[\left[P\left(K_{3}\right)^{(20)} \times P(K)^{(10)}\right]^{(11)} \times\left[P\left(K_{1}, K_{2}\right)^{(20)} \times P\left(K^{*}\right)^{(02)}\right]^{(11)}\right]_{00}^{(00)} \\
=\sqrt{\frac{1}{30}}\left(K_{3} \cdot K^{*}\right)\left\{\left(K_{1} \cdot K^{*}\right)\left(K_{2} \cdot\left[K_{3} \times K\right]\right)+\left(K_{2} \cdot K^{*}\right)\left(K_{1} \cdot\left[K_{3} \times K\right]\right)\right\} \\
\sqrt{15}\left[\left[P\left(K_{1}\right)^{(20)} \times P\left(K_{2}\right)^{(20)}\right]^{(21)} \times\left[P\left(K_{1}^{*}\right)^{(02)} \times P\left(K_{2}^{*}\right)^{(02)}\right]^{(12)}\right]_{00}^{(00)} \\
=\frac{1}{8}\left\{\left(K_{1} \cdot K_{1}^{*}\right)^{2}\left(K_{2} \cdot K_{2}^{*}\right)^{2}-\left(K_{1} \cdot K_{2}^{*}\right)^{2}\left(K_{2} \cdot K_{1}^{*}\right)^{2}\right\}
\end{gathered}
$$

\section{B.4. SAMPLE DERIVATIONS}

(i) Relation (B.5) can be proved by induction. For $n=1$ the validity follows from eq. (B.4), which is an expression of a simple SU(3) coupling. The SU(3) Wigner coefficients $\left\langle(10) \alpha_{1} ;(10) \alpha_{2} \mid(01) \gamma\right\rangle$ have the values $\pm \sqrt{\frac{1}{2}}$; with $\alpha=z, x, y$ corresponding to $\varepsilon A M_{A}=200,-1 \frac{1}{2}+\frac{1}{2},-1 \frac{1}{2}-\frac{1}{2}$, respectively. Using the pseudovector version of relation (B.1) we can express

$$
P\left(\left[K_{1} \times K_{2}\right]\right)_{\gamma}^{(0, n+1)}=\left[\frac{n ! 1 !}{(n+1) !}\right]^{\frac{1}{2}}\left[P\left(\left[K_{1} \times K_{2}\right]\right)^{(0 n)} \times P\left(\left[K_{1} \times K_{2}\right]^{(01)}\right]_{\gamma}^{(0, n+1)} .\right.
$$


Now, assuming the validity for $n$, (and for $n=1$ ), this can be written

$$
\begin{aligned}
P\left(\left[K_{1} \times K_{2}\right]\right)_{\gamma}^{(0, n+1)} & =\left[\frac{(n+1) ! 2 !}{(n+1)}\right]^{\frac{1}{2}}\left[\left[P\left(K_{1}\right)^{(n 0)} \times P\left(K_{2}\right)^{(n 0)}\right]^{(0 n)}\right. \\
& \left.\times\left[P\left(K_{1}\right)^{(10)} \times P\left(K_{2}\right)^{(10)}\right]^{(01)}\right]_{\gamma}^{(0, n+1)} \\
& =[n ! 2 !]^{\frac{1}{2}} \frac{(n+1) !}{n ! 1 !}\left[\begin{array}{lll}
(n 0) & (n 0) & (0 n) \\
(10) & (10) & (01) \\
(n+1,0) & (n+1,0) & (0, n+1)
\end{array}\right] \\
& \times\left[P\left(K_{1}\right)^{(n+1,0)} \times P\left(K_{2}\right)^{(n+1,0)}\right]_{\gamma}^{(0, n+1)},
\end{aligned}
$$

where we have used a 9-( $(\lambda \mu)$ recoupling transformation and the renormalization eq. (B.1) in the $K_{1}$ space and the $K_{2}$ space. The 9-( $\left.\lambda \mu\right)$ coefficient is equivalent to a simple 9- $j$ coefficient, [see eq. (A.21)]; and, with three $j$-values of 0 , has the simple value $[(n+2) / 2(n+1)]^{\frac{1}{2}}$. Eq. (B.5) follows.

(ii) The derivation of eq. (B.7) follows from simple SU(3) recoupling transformations. Using eq. (B.6) and (B.3)

$$
\begin{aligned}
& {\left[\left(\boldsymbol{K}_{1} \cdot \boldsymbol{K}_{2}^{*}\right)^{a}\left(\boldsymbol{K}_{1} \cdot \boldsymbol{K}_{1}^{*}\right)^{c}\right]\left(\boldsymbol{K}_{3} \cdot \boldsymbol{K}_{1}^{*}\right)^{b}=[a ! c !(a+c) ! d(a+c, 0) d(b, 0)]^{\frac{1}{b}} b !} \\
& \quad \times\left[\left[P\left(\boldsymbol{K}_{1}\right)^{(a+c, 0)} \times\left[P\left(\boldsymbol{K}_{2}^{*}\right)^{(0 a)} \times P\left(\boldsymbol{K}_{1}^{*}\right)^{(0 c)}\right]^{(0, a+c)}\right]^{(00)} \times\left[P\left(\boldsymbol{K}_{3}\right)^{(b 0)} \times P\left(\boldsymbol{K}_{1}^{*}\right)^{(0 b)}\right]^{(00)}\right]_{00}^{(00)} .
\end{aligned}
$$

A 9-( $\lambda \mu$ ) recoupling transformation, using the relation (A.16) for the recoupling coefficient with three $(00)$ representations, yields

$$
\begin{aligned}
& \left(K_{1} \cdot K_{2}^{*}\right)^{a}\left(K_{1} \cdot K_{1}^{*}\right)^{c}\left(K_{3} \cdot K_{1}^{*}\right)^{b}=\sum_{(\lambda \mu)}[a ! c !(a+c) ! d(\lambda \mu)]^{\frac{1}{t}} b ! \\
& \quad \times\left[\left[P\left(K_{1}\right)^{(a+c, 0)} \times P\left(K_{3}\right)^{(b 0)}\right]^{(\lambda \mu)} \times\left[\left[P\left(K_{2}^{*}\right)^{(0 a)} \times P\left(K_{1}^{*}\right)^{(0 c)}\right]^{(0, a+c)} \times P\left(K_{1}^{*}\right)^{(0 b)}\right]^{(\mu \lambda)}\right]_{00}^{(00)} .
\end{aligned}
$$

A 6- $(\lambda \mu)$ recoupling transformation on the $(\mu \lambda)$-function, together with the renormalization of the $K_{1}^{*}$ function, gives

$$
\begin{aligned}
& {\left[\left[P\left(K_{2}^{*}\right)^{(0 a)} \times P\left(K_{1}^{*}\right)^{(0 c)}\right]^{(0, a+c)} \times P\left(K_{1}^{*}\right)^{(0 b)}\right]_{\alpha}^{(\mu \lambda)}} \\
& \quad=\left[\frac{(b+c) !}{b ! c !}\right]^{*} U((0 a)(0 c)(\mu \lambda)(0 b) ;(0, a+c)(0, b+c))\left[P\left(K_{2}^{*}\right)^{(0 a)} \times P\left(K_{1}^{*}\right)^{(0, b+c)}\right]_{a}^{(\mu \lambda)}
\end{aligned}
$$

Use of the symmetry property (A.8) for the $U$-coefficient converts it to the form of eq. (A.12) and leads to the value of the coefficient of eq. (B.7).

(iii) The derivation of eq. (B.9) also follows from simple recoupling transformations. Using eq. (B.3) and the recoupling coefficient

$$
\begin{gathered}
U\left((00)(0 c)(00)(c 0) ;\left(\lambda^{\prime} \mu^{\prime}\right)(00)\right)=\delta_{\left(\lambda^{\prime} \mu^{\prime}\right)(0 c),} \\
\left(\boldsymbol{K} \cdot \boldsymbol{K}_{p}\right)^{a}\left(\boldsymbol{K}_{p} \cdot K_{p}^{*}\right)^{c}=\left[\left[\left[P(\boldsymbol{K})^{(a 0)} \times P\left(K_{p}\right)^{(0 a)}\right]^{(00)} \times P\left(K_{p}\right)^{(0 c)}\right]^{(0 c)} \times P\left(K_{p}^{*}\right)^{(c 0)}\right]_{00}^{(00)} .
\end{gathered}
$$

A 6-( $(\lambda \mu)$ transformation, using the special value (A.7) for the multiplicity-free $U$-coef- 
ficient, together with the renormalization of the $K_{\mathrm{p}}$ space functions, yields

$$
\begin{aligned}
\left(\boldsymbol{K} \cdot \boldsymbol{K}_{\mathrm{p}}\right)^{a}\left(\boldsymbol{K}_{\mathrm{p}} \cdot \boldsymbol{K}_{\mathrm{p}}^{*}\right)^{c}=\left[\frac{d(0, a+c)}{d(a, 0) d(0, c)}\right]^{ \pm}\left[\frac{(a+c) !}{a ! c !}\right]^{\frac{1}{2}} \\
\times\left[\left[P(\boldsymbol{K})^{(a 0)} \times P\left(\boldsymbol{K}_{p}\right)^{(0, a+c)}\right]^{(0 c)} \times P\left(\boldsymbol{K}_{p}^{*}\right)^{(c 0)}\right]_{00}^{(00)} .
\end{aligned}
$$

Combination with the analogous expression for the two remaining factors gives

$$
\begin{aligned}
\left(\boldsymbol{K} \cdot \boldsymbol{K}_{\mathrm{p}}\right)^{a}\left(\boldsymbol{K}_{\mathrm{p}} \cdot \boldsymbol{K}_{\mathrm{p}}^{*}\right)^{c}\left(\boldsymbol{K} \cdot \boldsymbol{K}^{*}\right)^{e}\left(\boldsymbol{K}^{*} \cdot \boldsymbol{K}_{\mathrm{p}}^{*}\right)^{b} \\
=\left[\frac{d(0, a+c)}{d(a 0) d(0 c)}\right]^{t}\left[\frac{(a+c) !}{a ! c !}\right]^{t}\left[\frac{d(0, e+b)}{d(0 e) d(b 0)}\right]^{t}\left[\frac{(e+b) !}{e ! b !}\right]^{\frac{1}{2}} \\
\times\left[\left[\left[P(\boldsymbol{K})^{(u 0)} \times P\left(\boldsymbol{K}_{p}\right)^{(0, a+c)}\right]^{(0 c)} \times P\left(\boldsymbol{K}_{p}^{*}\right)^{(00)}\right]^{(00)}\right. \\
\left.\times\left[P(\boldsymbol{K})^{(e 0)} \times\left[P\left(K^{*}\right)^{(0, e+b)} \times P\left(\boldsymbol{K}_{p}^{*}\right)^{(b 0)}\right]^{(0 e)}\right]^{(00)}\right]_{00}^{(00)} .
\end{aligned}
$$

The $K$ and $K^{*}$ functions in the SU(3) coupled $P$ 's can now be brought together by the trivial $9-(\lambda \mu)$ recoupling transformation with three $(00)$ representations, eq. (A.16), to yield

$$
\begin{aligned}
{\left[[\ldots]^{(00)} \times[\ldots]^{(00)}\right]^{(00)} } & =\sum_{(\lambda \mu)}\left[\frac{d(\lambda \mu)}{d(c 0) d(0 e)}\right]^{\frac{1}{2}} \\
& \times\left[\left[\left[P(\boldsymbol{K})^{(a 0)} \times P\left(K_{\mathrm{p}}\right)^{(0, a+c)}\right]^{(0 c)} \times P(\boldsymbol{K})^{(e 0)}\right]^{(\lambda \mu)}\right. \\
& \left.\left.\times P\left(\boldsymbol{K}_{\mathrm{p}}^{*}\right)^{(\boldsymbol{c o )}} \times\left[P\left(\boldsymbol{K}^{*}\right)^{(0, e+b)} \times P\left(K_{\mathrm{p}}^{*}\right)^{(b 0)}\right]^{(0 e)}\right]^{(\mu \lambda)}\right]_{00}^{(00)} .
\end{aligned}
$$

A $6-(\lambda \mu)$ transformation in the $(\lambda \mu)$ function as well as in the $(\mu \lambda)$ function is then necessary to combine the $P(K)$ and the $P\left(K_{\mathrm{p}}^{*}\right)$ :

$$
\begin{aligned}
{\left[\left[P(\boldsymbol{K})^{(a 0)} \times P\left(\boldsymbol{K}_{p}\right)^{(0, a+c)}\right]^{(0 c)}\right.} & \left.\times P(\boldsymbol{K})^{(e 0)}\right]_{\alpha}^{(\lambda \mu)}=U((0, a+c)(a 0)(\lambda \mu)(e 0) ;(0 c)(a+e, 0)) \\
\times & {\left[\frac{(a+e) !}{a ! e !}\right]^{\frac{1}{2}}\left[P\left(\boldsymbol{K}_{p}\right)^{(0, a+c)} \times P(\boldsymbol{K})^{(a+e, 0)}\right]_{\alpha}^{(\lambda \mu)}, }
\end{aligned}
$$

where we have first interchanged the order of the $(a 0) \times(0, a+c)$ coupling. Note: For this multiplicity-free coupling this interchange is trivial and merly introduces the phase factor $(-1)^{a+a+c+c}=+1$, see eqs. (A.3a) and (A.3b). Similarly

$$
\begin{aligned}
& {\left[P\left(K_{\mathrm{p}}^{*}\right)^{(\boldsymbol{c o )}} \times\left[P\left(\boldsymbol{K}^{*}\right)^{(0, b+e)} \times\right.\right.}\left.\left.P\left(\boldsymbol{K}_{\mathrm{p}}^{*}\right)^{(b 0)}\right]^{(0 e)}\right]_{\beta}^{(\mu \lambda)}=U((c 0)(b 0)(\mu \lambda)(0, b+e) ;(b+c, 0)(0 e)) \\
& \times\left[\frac{(b+c) !}{b ! c !}\right]^{t}\left[P\left(K_{\mathrm{p}}^{*}\right)^{(b+c, 0)} \times P\left(K^{*}\right)^{(0, b+e)}\right]_{\beta}^{(\mu \lambda)}
\end{aligned}
$$

The two $U$-coefficients are evaluated through eqs. (A.13) and (A.14), respectively. The combination of all the factors gives the desired result, eq. (B.9). 


\section{Appendix C}

SINGLE-COLUMN BS TRANSFORMS OF NORM AND OVERLAP KERNELS

A tabulation of the BS transforms of norm and overlap matrix elements for totally antisymmetric spacc functions, (space functions characterized by single-columned Young tableaux), is given in this appendix.

\section{DEFINITIONS}

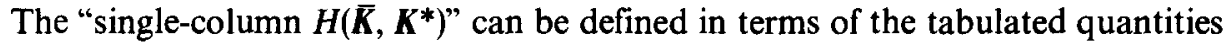
$H\left(\begin{array}{l}\left(\lambda_{c} \mu_{c}\right) \mu^{\prime}\left(\lambda^{\prime} \mu^{\prime}\right) \\ n^{\prime}\end{array}\right)$ by

$$
H\left(\overline{\boldsymbol{K}}, K^{*}\right)^{\left(\lambda_{c} \mu_{c}\right)\left(\lambda_{c}^{\prime} \mu_{c}^{\prime}\right)}=\mathrm{e}^{\eta\left(\overline{\mathbf{K}} \cdot \mathbf{K}^{*}\right)} H\left({ }_{n}^{l\left(\lambda_{c} \mu_{c}\right) l^{\prime} l^{\prime}\left(\lambda_{c}^{\prime} \mu_{c}^{\prime}\right)}\right),
$$

where $\bar{K}$ refers to the relative motion degree of freedom of fragments $f$ and $A-f$; while $K^{*}$ refers to the relative motion degree of freedom of fragments $f^{\prime}$ and $A-f^{\prime}$; $\bar{K}_{i}$ refers to the internal degrees of freedom of fragments $f$ and $A-f ; K_{i}^{*}$ refers to the internal degrees of freedom of fragments $f^{\prime}$ and $A-f^{\prime}$. Note that $f^{\prime}$ is chosen such that $f^{\prime} \leqq f$.

The indices $i$ are illustrated in figs. $1 \mathrm{a}$ and $1 \mathrm{~b}$.

The quantities $e^{p}$ are defined by

$$
e^{p} \equiv \exp \left\{-p \frac{A}{\left[f(A-f) f^{\prime}\left(A-f^{\prime}\right)\right]^{\frac{1}{2}}}\left(\overline{\boldsymbol{K}} \cdot \boldsymbol{K}^{*}\right)\right\} .
$$

Note that $e^{p} e^{q}=e^{p+q}$. The only important property of the mass-dependent factor $\eta$ is

$$
\sum_{\text {columns }} \eta=\left[\frac{f^{\prime}(A-f)}{f\left(A-f^{\prime}\right)}\right]^{\frac{1}{2}}
$$

i.e., for the full 4-columned Young tableaux, the product of factors $\exp \eta\left(\overline{\boldsymbol{K}} \cdot \boldsymbol{K}^{*}\right)$ combine to make the factor

$$
\prod_{\text {columns }} \mathrm{c}^{\eta\left(\overline{\mathbf{K}} \cdot \mathbf{K}^{*}\right)}=\exp \left\{\frac{f^{\prime}(A-f)}{\left[f(A-f) f^{\prime}\left(A-f^{\prime}\right)\right]^{\frac{4}{2}}}\left(\overline{\mathbf{K}} \cdot \boldsymbol{K}^{*}\right)\right\} .
$$

[Specifically,

$$
\eta=\frac{l^{\prime}(A-f)-l f^{\prime}+(l+n)\left(f f^{\prime} / A\right)}{\left[f(A-f) f^{\prime}\left(A-f^{\prime}\right)\right]^{\frac{1}{2}}},
$$

with $\sum l^{\prime}=f^{\prime}, \sum l=f, \sum(l+n)=A$. $]$

In the special case, $f^{\prime}=f$, (norm kernels)

$$
H\left(\overline{\boldsymbol{K}}, \boldsymbol{K}^{*}\right)^{\left(\lambda_{c} \mu_{c}\right)\left(\lambda_{c} \mu_{c}\right)}=\mathrm{e}^{\eta\left(\overline{\mathbf{K}} \cdot \boldsymbol{K}^{*}\right)} H\left({ }_{n}^{l\left(\lambda_{c} \mu_{c}\right) l\left(\lambda_{c} \mu_{c}\right)}\right),
$$

with

$$
\begin{gathered}
e^{p} \equiv \exp \left\{-p \frac{A}{f(A-f)}\left(\overline{\mathbf{K}} \cdot \boldsymbol{K}^{*}\right)\right\}, \\
\sum \eta=1
\end{gathered}
$$


1. $H\left({ }_{n}^{0\left(\lambda_{c} \mu_{c}\right) O\left(\lambda_{c} \mu_{c}\right)}\right)$ (purely internal functions).

$H\left({ }_{1}^{(00)} \underset{1}{(00)}\right)=1$,

$H(\underset{2}{0(10) 0(10)})=\left(\bar{K}_{1} \cdot K_{1}^{*}\right)$,

$H\left({ }_{3}^{(01)(01)}\right)=\left(\overline{\boldsymbol{K}}_{12} \cdot \boldsymbol{K}_{12}^{*}\right) \quad$ where $\overline{\boldsymbol{K}}_{12}=\frac{1}{\sqrt{2}}\left[\overline{\boldsymbol{K}}_{1} \times \overline{\boldsymbol{K}}_{2}\right]$,

$H\left({ }_{4}^{(00) 0(00)}\right)=P_{123}^{(00)} \equiv \frac{1}{6}\left(\left[\bar{K}_{1} \times \bar{K}_{2}\right] \cdot \bar{K}_{3}\right)\left(\left[K_{1}^{*} \times K_{2}^{*}\right] \cdot K_{3}^{*}\right)^{a)}$,

$H\left({ }_{5}^{0(20) 0(20)}\right)=\frac{1}{2}\left(\bar{K}_{4} \cdot K_{4}^{*}\right)^{2, a)}$

[Shorthand notation for $\frac{1}{2}\left(\bar{K}_{4} \cdot K_{4}^{*}\right)^{2} \times P_{123}^{(00)}$ ]

$H\left({ }_{6}^{0(21)} \underset{6}{(21)}\right)=\frac{1}{8}\left\{\left(\bar{K}_{4} \cdot K_{4}^{*}\right)^{2}\left(\bar{K}_{5} \cdot K_{5}^{*}\right)^{2}-\left(\bar{K}_{4} \cdot K_{5}^{*}\right)^{2}\left(\bar{K}_{5} \cdot K_{4}^{*}\right)^{2}\right\}{ }^{a)}$

\section{2. $H\left({ }_{n}^{1\left(\lambda_{c} \mu_{c}\right) 1\left(\lambda_{c} \mu_{c}\right)}\right)$}

$$
\begin{aligned}
& H\left({ }_{1}^{1(00) 1(00)}\right)=\left(1-e^{1}\right) \text {, } \\
& H(\underset{2}{1(10) 1(10)})=\left\{\left(1-e^{1}\right)\left(\overline{\boldsymbol{K}}_{1} \cdot \boldsymbol{K}_{1}^{*}\right)-e^{1} \frac{A}{f(A-f)}\left(\overline{\boldsymbol{K}} \cdot \boldsymbol{K}_{1}^{*}\right)\left(\overline{\boldsymbol{K}}_{1} \cdot \boldsymbol{K}^{*}\right)\right\}, \\
& H\left(3_{3}^{(01) 1(01)}\right)=\left\{\left(1-e^{1}\right)\left(\overline{\boldsymbol{K}}_{12} \cdot \boldsymbol{K}_{12}^{*}\right)-e^{1} \frac{A}{f(A-f)}\left[\left(\overline{\mathbf{K}} \cdot \boldsymbol{K}^{*}\right)\left(\overline{\boldsymbol{K}}_{12} \cdot \boldsymbol{K}_{12}^{*}\right)\right.\right. \\
& \left.\left.-\left(\bar{K} \cdot \bar{K}_{12}\right)\left(K^{*} \cdot K_{12}^{*}\right)\right]\right\},
\end{aligned}
$$

$$
\begin{aligned}
H\left({ }_{4}^{1(00) 1(00)}\right)= & P_{123}^{(00)}\left\{\left(1-e^{1}\right)-e^{1} \frac{A}{f(A-f)}\left(\overline{\boldsymbol{K}} \cdot \boldsymbol{K}^{*}\right)\right\} \\
H\left({ }_{5}^{1(20) 1(20)}\right)= & \frac{1}{2}\left\{\left[\left(1-e^{1}\right)-e^{1} \frac{A}{f(A-f)}\left(\overline{\mathbf{K}} \cdot \boldsymbol{K}^{*}\right)\right]\left(\overline{\boldsymbol{K}}_{4} \cdot \boldsymbol{K}_{4}^{*}\right)^{2}\right. \\
& \left.-\frac{1}{2} e^{1}\left(\frac{A}{f(A-f)}\right)^{2}\left(\overline{\boldsymbol{K}} \cdot \boldsymbol{K}_{4}^{*}\right)^{2}\left(\overline{\boldsymbol{K}}_{4} \cdot \boldsymbol{K}^{*}\right)^{2}\right\},
\end{aligned}
$$

$$
\begin{aligned}
& H\left({ }_{6}^{1(21) 1(21)}\right)=\frac{1}{8}\left\{[ ( 1 - e ^ { 1 } ) - e ^ { 1 } \frac { A } { f ( A - f ) } ( \overline { \boldsymbol { K } } \cdot \boldsymbol { K } ^ { * } ) ] \left[\left(\overline{\boldsymbol{K}}_{4} \cdot \boldsymbol{K}_{4}^{*}\right)^{2}\left(\overline{\boldsymbol{K}}_{5} \cdot \boldsymbol{K}_{5}^{*}\right)^{2}\right.\right. \\
& -\left(\overline{\boldsymbol{K}}_{4} \cdot \boldsymbol{K}_{5}^{*}\right)^{2}\left(\overline{\boldsymbol{K}}_{5} \cdot \boldsymbol{K}_{4}^{*}\right)^{2}-\frac{1}{2} e^{1}\left(\frac{A}{f(A-f)}\right)^{2}\left[\left(\overline{\boldsymbol{K}} \cdot \boldsymbol{K}_{4}^{*}\right)^{2}\left(\overline{\boldsymbol{K}}_{4} \cdot \boldsymbol{K}^{*}\right)^{2}\left(\overline{\boldsymbol{K}}_{5} \cdot \boldsymbol{K}_{5}^{*}\right)^{2}\right. \\
& +\left(\overline{\boldsymbol{K}} \cdot \boldsymbol{K}_{5}^{*}\right)^{2}\left(\overline{\boldsymbol{K}}_{5} \cdot \boldsymbol{K}^{*}\right)^{2}\left(\overline{\boldsymbol{K}}_{4} \cdot \boldsymbol{K}_{4}^{*}\right)^{2}-\left(\overline{\boldsymbol{K}} \cdot \boldsymbol{K}_{5}^{*}\right)^{2}\left(\overline{\boldsymbol{K}}_{4} \cdot \boldsymbol{K}^{*}\right)^{2}\left(\overline{\boldsymbol{K}}_{5} \cdot \boldsymbol{K}_{4}^{*}\right)^{2} \\
& \left.\left.-\left(\overline{\boldsymbol{K}} \cdot \boldsymbol{K}_{4}^{*}\right)^{2}\left(\overline{\boldsymbol{K}}_{5} \cdot \boldsymbol{K}^{*}\right)^{2}\left(\overline{\boldsymbol{K}}_{4} \cdot \boldsymbol{K}_{5}^{*}\right)^{2}\right]\right\}
\end{aligned}
$$

2) This SU(3) scalar will usually be suppressed in an obvious shorthand notation. 
3. $H\left(\begin{array}{l}l\left(\lambda_{c} \mu_{c}\right) l^{\prime}\left(\lambda_{n^{\prime}}^{\prime} \mu_{c}^{\prime}\right) \\ n^{\prime}\end{array}\right)$, needed for the $A=24$ system

$$
\begin{aligned}
& H\left({\underset{4}{4}}_{4}^{2(10) 2(10)}\right)=\left\{\left(1-e^{1}\right)^{2}\left(\overline{\boldsymbol{K}}_{1} \cdot \boldsymbol{K}_{1}^{*}\right)-e^{1}\left(1-e^{1}\right) \frac{A}{f(A-f)}\left[\left(\overline{\boldsymbol{K}} \cdot \boldsymbol{K}^{*}\right)\left(\overline{\boldsymbol{K}}_{1} \cdot \boldsymbol{K}_{1}^{*}\right)\right.\right. \\
& \left.\left.-\left(\overline{\boldsymbol{K}} \cdot \boldsymbol{K}_{1}^{*}\right)\left(\overline{\boldsymbol{K}}_{1} \cdot \boldsymbol{K}^{*}\right)\right]-e^{1}\left(\frac{A}{f(A-f)}\right)^{2}\left(\overline{\boldsymbol{K}} \cdot \boldsymbol{K}^{*}\right)\left(\overline{\boldsymbol{K}} \cdot \boldsymbol{K}_{1}^{*}\right)\left(\overline{\boldsymbol{K}}_{1} \cdot \boldsymbol{K}^{*}\right)\right\} P_{234}^{(00)}, \\
& H\left({ }_{3}^{3(02) 3(02)}\right)=\left\{\frac{1}{2}\left[\left(1-e^{1}\right)^{3}-\left(e^{1}-e^{2}\right)\left(\frac{A}{f(A-f)}\right)^{2}\left(\overline{\boldsymbol{K}} \cdot \boldsymbol{K}^{*}\right)^{2}\right]\right. \\
& \quad \times\left[\left(\overline{\boldsymbol{K}}_{12} \cdot \boldsymbol{K}_{12}^{*}\right)\left(\overline{\boldsymbol{K}}_{34} \cdot \boldsymbol{K}_{34}^{*}\right)+\left(\overline{\boldsymbol{K}}_{12} \cdot \boldsymbol{K}_{34}^{*}\right)\left(\overline{\boldsymbol{K}}_{34} \cdot \boldsymbol{K}_{12}^{*}\right)\right] \\
& +\left(e^{1}-e^{2}\right)\left(\frac{A}{f(A-f)}\right)^{2}\left(\frac { 1 } { 2 } ( \overline { \boldsymbol { K } } \cdot \boldsymbol { K } ^ { * } ) \left[\left(\overline{\boldsymbol{K}}_{12} \cdot \boldsymbol{K}_{12}^{*}\right)\left(\overline{\boldsymbol{K}} \cdot \overline{\boldsymbol{K}}_{34}\right)\left(\boldsymbol{K}^{*} \cdot \boldsymbol{K}_{34}^{*}\right)\right.\right. \\
& +\left(\overline{\boldsymbol{K}}_{34} \cdot \boldsymbol{K}_{34}^{*}\right)\left(\overline{\boldsymbol{K}} \cdot \overline{\boldsymbol{K}}_{12}\right)\left(\boldsymbol{K}^{*} \cdot \boldsymbol{K}_{12}^{*}\right)+\left(\overline{\boldsymbol{K}}_{12} \cdot \boldsymbol{K}_{34}^{*}\right)\left(\overline{\boldsymbol{K}} \cdot \overline{\boldsymbol{K}}_{34}\right)\left(\boldsymbol{K}^{*} \cdot \boldsymbol{K}_{12}^{*}\right) \\
& \left.\left.\left.+\left(\overline{\boldsymbol{K}}_{34} \cdot \boldsymbol{K}_{\mathbf{1}}^{*}\right)\left(\overline{\boldsymbol{K}} \cdot \overline{\boldsymbol{K}}_{12}\right)\left(\boldsymbol{K}^{*} \cdot \boldsymbol{K}_{34}^{*}\right)\right]-\left(\overline{\boldsymbol{K}} \cdot \overline{\boldsymbol{K}}_{12}\right)\left(\overline{\boldsymbol{K}} \cdot \overline{\boldsymbol{K}}_{34}\right)\left(\boldsymbol{K}^{*} \cdot \boldsymbol{K}_{12}^{*}\right)\left(\boldsymbol{K}^{*} \cdot \boldsymbol{K}_{34}^{*}\right)\right)\right\},
\end{aligned}
$$

where

$$
\overline{\boldsymbol{K}}_{12}=\frac{1}{\sqrt{2}}\left[\overline{\boldsymbol{K}}_{1} \times \overline{\boldsymbol{K}}_{2}\right], \quad \overline{\boldsymbol{K}}_{34}=\frac{1}{\sqrt{2}}\left[\overline{\boldsymbol{K}}_{3} \times \overline{\boldsymbol{K}}_{4}\right],
$$

$$
\begin{aligned}
& H\left({ }_{3}^{(02) 3(10)}\right)=0 \text {, } \\
& H\left({ }_{3}^{(30)} \underset{3}{3(10)}\right)=\left\{\left(1-e^{1}\right)^{2}\left(1+e^{1}\right)\left(\bar{K}_{i} \cdot K_{i}^{*}\right)\right. \\
& -2 e^{1}\left(1-e^{1}\right) \frac{A}{f(A-f)}\left[\left(\overline{\boldsymbol{K}} \cdot \boldsymbol{K}^{*}\right)\left(\overline{\boldsymbol{K}}_{i} \cdot \boldsymbol{K}_{i}^{*}\right)-\left(\overline{\boldsymbol{K}} \cdot \boldsymbol{K}_{i}^{*}\right)\left(\overline{\boldsymbol{K}}_{i} \cdot \boldsymbol{K}^{*}\right)\right] \\
& \left.-e^{1}\left(1+e^{1}\right)\left(\frac{A}{f(A-f)}\right)^{2}\left(\overline{\boldsymbol{K}} \cdot \boldsymbol{K}^{*}\right)\left(\overline{\boldsymbol{K}} \cdot \boldsymbol{K}_{i}^{*}\right)\left(\overline{\boldsymbol{K}}_{i} \cdot K^{*}\right)\right\},
\end{aligned}
$$

where

$$
\begin{aligned}
& \overline{\boldsymbol{K}}_{i}=\sqrt{ } \frac{1}{2}\left[\overline{\boldsymbol{K}}_{12} \times \overline{\boldsymbol{K}}_{34}\right] \\
& H\left({ }_{3}^{3(02) 2(10)}\right)=-\frac{1}{2}\left(1-e^{1}\right)^{2} \frac{A}{\left[f(A-f) f^{\prime}\left(A-f^{\prime}\right)\right]^{\frac{1}{2}}} \\
& \left\{\left(\overline{\boldsymbol{K}} \cdot \overline{\boldsymbol{K}}_{12}\right)\left(\boldsymbol{K}^{*} \cdot\left[\overline{\boldsymbol{K}}_{34} \times \boldsymbol{K}_{1}^{*}\right]\right)+\left(\overline{\boldsymbol{K}}_{34} \cdot \overline{\boldsymbol{K}}\right)\left(\boldsymbol{K}^{*} \cdot\left[\overline{\boldsymbol{K}}_{12} \times \boldsymbol{K}_{1}^{*}\right]\right)\right\}, \\
& H\left({ }_{3}^{3(10) 2(10)}\right)=\left\{-\left(1-e^{1}\right)^{2}\left(\left[\bar{K}_{12} \times \bar{K}_{34}\right] \cdot K_{1}^{*}\right)\right. \\
& +\frac{1}{2}\left(1-e^{2}\right) \frac{A}{\left[f(A-f) f^{\prime}\left(A-f^{\prime}\right)\right]^{\frac{1}{2}}}\left[\left(\overline{\mathbf{K}} \cdot \boldsymbol{K}^{*}\right)\left(\left[\overline{\boldsymbol{K}}_{12} \times \overline{\boldsymbol{K}}_{34}\right] \cdot \boldsymbol{K}_{1}^{*}\right)-\left(\overline{\mathbf{K}} \cdot \boldsymbol{K}_{1}^{*}\right)\right. \\
& \left.\times\left(\left[\overline{\mathbf{K}}_{12} \times \overline{\mathbf{K}}_{34}\right] \cdot \mathbf{K}^{*}\right)\right]+e^{1} \frac{A^{2}}{f(A-f) f^{\prime}\left(A-f^{\prime}\right)}\left(\overline{\mathbf{K}} \cdot \boldsymbol{K}^{*}\right)\left[\left(\overline{\mathbf{K}} \cdot \boldsymbol{K}_{1}^{*}\right)\left(\left[\overline{\boldsymbol{K}}_{12} \times \overline{\boldsymbol{K}}_{34}\right] \cdot \boldsymbol{K}^{*}\right)\right\},
\end{aligned}
$$




$$
\begin{aligned}
& H\left(3_{3}^{3(02))(20)}\right)=\frac{1}{2}\left(1-e^{1}\right)\left[\frac{A}{f(A-f)}\right]^{\frac{1}{2}} \frac{A}{\left[f(A-f) f^{\prime}\left(A-f^{\prime}\right)\right]^{\frac{1}{2}}}\left(\overline{\mathbf{K}} \cdot \boldsymbol{K}_{4}^{*}\right) \\
& \times\left\{\left(\overline{\boldsymbol{K}} \cdot \overline{\boldsymbol{K}}_{12}\right)\left(\boldsymbol{K}_{4}^{*} \cdot\left[\boldsymbol{K}^{*} \times \overline{\boldsymbol{K}}_{34}\right]\right)+\left(\overline{\boldsymbol{K}} \cdot \overline{\boldsymbol{K}}_{34}\right)\left(\boldsymbol{K}_{\mathbf{4}}^{*} \cdot\left[\boldsymbol{K}^{*} \times \overline{\boldsymbol{K}}_{12}\right]\right)\right\}, \\
& H\left({ }_{3}^{3(10) 1(20)}\right)=\left[\frac{A}{f(A-f)}\right]^{\frac{*}{2}}\left(\overline{\mathbf{K}} \cdot \boldsymbol{K}_{4}^{*}\right)\left(\left[\overline{\boldsymbol{K}}_{12} \times \overline{\mathbf{K}}_{34}\right] \cdot \boldsymbol{K}_{4}^{*}\right) \\
& \times\left[\left(1-e^{1}\right)-\frac{1}{2}\left(1+e^{1}\right)\left(\bar{K} \cdot K^{*}\right) \frac{A}{\left[f(A-f) f^{\prime}\left(A-f^{\prime}\right)\right]^{\frac{1}{2}}}\right], \\
& \left.H\left({ }_{4}^{2(10)}\right)_{5}^{(20)}\right)=\left[\frac{A}{f(A-f)}\right]^{\frac{1}{2}}\left\{\left(1-e^{1}\right)\left(\bar{K}_{1} \cdot K_{4}^{*}\right)\left(\bar{K} \cdot K_{4}^{*}\right)-\frac{A}{\left[f(A-f) f^{\prime}\left(A-f^{\prime}\right)\right]^{\frac{1}{2}}}\right. \\
& \left.\times\left[\frac{1}{2}\left(1-e^{1}\right)\left(\overline{\boldsymbol{K}}_{1} \cdot \boldsymbol{K}^{*}\right)\left(\overline{\boldsymbol{K}} \cdot \boldsymbol{K}_{4}^{*}\right)^{2}+e^{1}\left(\overline{\boldsymbol{K}} \cdot \boldsymbol{K}^{*}\right)\left(\overline{\boldsymbol{K}} \cdot \boldsymbol{K}_{4}^{*}\right)\left(\overline{\boldsymbol{K}}_{1} \cdot \boldsymbol{K}_{4}^{*}\right)\right]\right\}, \\
& H\left({ }_{3}^{3(10) 0(21)}\right)=0 \text {, } \\
& H(\underset{3}{3(02) 0(21)})=\frac{1}{2 \sqrt{2}}\left[\frac{A}{f(A-f)}\right]^{\frac{3}{2}}\left(\overline{\mathbf{K}} \cdot \boldsymbol{K}_{4}^{*}\right)\left(\bar{K} \cdot K_{5}^{*}\right) \\
& \times\left\{\left[\overline{\boldsymbol{K}} \cdot \overline{\boldsymbol{K}}_{12}\right)\left(\overline{\boldsymbol{K}}_{34} \cdot\left[\boldsymbol{K}_{4}^{*} \times \boldsymbol{K}_{5}^{*}\right]\right)+\left(\overline{\boldsymbol{K}} \cdot \overline{\boldsymbol{K}}_{\mathbf{3 4}}\right)\left(\overline{\boldsymbol{K}}_{12} \cdot\left[\boldsymbol{K}_{4}^{*} \times \boldsymbol{K}_{5}^{*}\right]\right)\right\}, \\
& H\left({ }_{3}^{3(02))(22)}\right)=\frac{A}{f(A-f)} \sqrt{27}\left\{\left[-2\left(1-e^{1}\right)+\left(1+e^{1}\right)\left(\bar{K} \cdot K^{*}\right) \frac{A}{\left[f(A-f) f^{\prime}\left(A-f^{\prime}\right)\right]^{\frac{1}{2}}}\right]\right. \\
& {\left[\mathscr{R}^{(22)}\left(\bar{K}_{12} \vec{K}_{34} \bar{K}\right) \times \mathscr{P}^{(22)}\left(K_{12}^{*} K_{3}^{*} K_{4}^{*}\right)\right]_{00}^{(00)}-\frac{1}{2}\left(1+e^{1}\right) \frac{A}{\left[f(A-f) f^{\prime}\left(A-f^{\prime}\right)\right]^{\frac{1}{2}}}} \\
& \times\left[\left(\bar{K} \cdot \bar{K}_{12}\right)\left[\mathscr{R}^{(22)}\left(K^{*} \bar{K}_{34} \bar{K}\right) \times \mathscr{P}^{(22)}\left(K_{12}^{*} K_{3}^{*} K_{4}^{*}\right)\right]_{00}^{(00)}\right. \\
& \left.\left.+\left(\bar{K} \cdot \mathbb{K}_{34}\right)\left[\mathscr{R}^{(22)}\left(\boldsymbol{K}^{*} \bar{K}_{12} \bar{K}\right) \times \mathscr{P}^{(22)}\left(\boldsymbol{K}_{12}^{*} \boldsymbol{K}_{3}^{*} \boldsymbol{K}_{4}^{*}\right)\right]_{00}^{(00)}\right]\right\},
\end{aligned}
$$

where

$$
\begin{aligned}
& \mathscr{R}^{(22)}\left(\bar{K}_{12} \bar{K}_{34} \bar{K}\right) \equiv\left[\left[P^{(01)}\left(\bar{K}_{12}\right) \times P^{(01)}\left(\bar{K}_{34}\right)\right]^{(02)} \times P(\bar{K})^{(20)}\right]^{(22)} \\
& \mathscr{P}^{(22)}\left(K_{12}^{*} K_{3}^{*} K_{4}^{*}\right) \equiv\left[P\left(K_{12}^{*}\right)^{(10)} \times\left[P\left(K_{3}^{*}\right)^{(02)}+P\left(K_{4}^{*}\right)^{(02)}\right]^{(12)}\right]^{(22)}, \\
& H\left({ }_{3}^{3(10) 1(22)}\right)=3 \sqrt{6}\left[\frac{A}{f(A-f)}\right]_{5}^{\frac{z}{3}}\left[\frac{A}{f^{\prime}\left(A-f^{\prime}\right)}\right]^{\frac{1}{2}}\left(1-e^{1}\right) \\
& \times\left[\left[\left[P\left(\bar{K}_{12}\right)^{(01)} \times P\left(\bar{K}_{34}\right)^{(01)}\right]^{(10)} \times P(\bar{K})^{(30)}\right]^{(21)}\right. \\
& \left.\times\left[\mathscr{P}^{(22)}\left(K_{12}^{*} K_{3}^{*} K_{4}^{*}\right) \times P^{(01)}\left(K^{*}\right)\right]^{(12)}\right]_{00}^{(00)}
\end{aligned}
$$




\section{Appendix D}

The single-column BS transforms are calculated with the aid of the functions

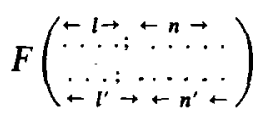

defined in terms of the operations of eq. (56) acting on $(l+n)$ particle antisymmetrizers. Particles $1, \ldots, l$ belong to fragment $f$, particles $l+1, \ldots, l+n$ to fragment $A-f$;

The $f$-functions with $l=l^{\prime}, n=n^{\prime}$

\begin{tabular}{|c|c|}
\hline$f\left(\begin{array}{l}0 ; 0 \\
0 ; 0\end{array}\right)$ & $\left(1-e^{1}\right)$ \\
\hline$f\left(\begin{array}{c}0 ; 0 \bar{z} \\
0 ; 0 z^{*}\end{array}\right)$ & $1-e^{1}\left(1+\beta_{z z}\right)$ \\
\hline$f\left(\begin{array}{l}0 ; 0 \bar{z} \\
0 ; 0 x^{*}\end{array}\right)$ & $-e^{1} \beta_{x z}$ \\
\hline$f\left(\begin{array}{c}0 ; 0 \bar{x} \\
0 ; 0 z^{*}\end{array}\right)$ & $-e^{1} \beta_{z x}$ \\
\hline$f\left(\begin{array}{l}0 ; 0 \bar{z} \bar{x} \\
0 ; 0 z^{*} x^{*}\end{array}\right)$ & $1-e^{1}\left(1+\beta_{z z}+\beta_{x x}\right)$ \\
\hline$f\left(\begin{array}{c}0 \bar{z} ; 0 \bar{z} \\
0 z^{*} ; 0 z^{*}\end{array}\right)$ & $1-e^{1}\left(2+\beta_{z z}^{2}\right)+e^{2}$ \\
\hline$f\left(\begin{array}{l}0, \bar{x} ; 0 \bar{z} \\
0 x^{*} ; 0 z^{*}\end{array}\right)$ & $1-e^{1}\left(1+\beta_{z z}+\beta_{x x}+\beta_{z z} \beta_{x x}\right)$ \\
\hline$f\left(\begin{array}{c}0 \bar{z} ; 0 \bar{x} \\
0 z^{*} ; 0 x^{*}\end{array}\right)$ & $1-e^{1}\left(1+\beta_{z z}+\beta_{x x}+\beta_{z z} \beta_{x x}\right)$ \\
\hline$f\left(\begin{array}{l}0 \bar{z} ; 0 \bar{x} \\
0 x^{*} ; 0 z^{*}\end{array}\right)$ & $-e^{1}\left(1-\beta_{z z}-\beta_{x x}+\beta_{z z} \beta_{x x}\right)+e^{2}$ \\
\hline$f\left(\begin{array}{c}0 \bar{x} ; 0 \bar{z} \\
0 z^{*} ; 0 x^{*}\end{array}\right)$ & $-e^{1}\left(1-\beta_{z z}-\beta_{x x}+\beta_{z z} \beta_{x x}\right)+e^{2}$ \\
\hline$f\left(\begin{array}{l}0 \bar{x} ; 0 \bar{z} \\
0 z^{*} ; 0 z^{*}\end{array}\right)$ & $-e^{1} \beta_{z x} \beta_{z z}$ \\
\hline$f\left(\begin{array}{l}0 \bar{z} ; 0 \bar{z} \\
0 x^{*} ; 0 z^{*}\end{array}\right)$ & $-e^{1} \beta_{x z} \beta_{z z}$ \\
\hline$f\left(\begin{array}{l}0 x^{*} ; 0 x^{*} \\
0 \bar{z}\end{array}\right)$ & $-e^{1} \beta_{x z}^{2}$ \\
\hline$f\left(\begin{array}{l}0 \bar{x} ; 0 \bar{x} \\
0 z^{*} ; 0 z^{*}\end{array}\right)$ & $-e^{1} \beta_{z x}^{2}$ \\
\hline$f\left(\begin{array}{ll}0 \bar{z} \bar{x} ; 0 \bar{z} \bar{x} \\
0 z^{*} x^{*} ; 0 z^{*} x^{*}\end{array}\right)$ & $\left\{1-e^{1}\left(3+\left[\beta_{x x}+\beta_{z z}\right]^{2}\right)+e^{2}\left(3+\left[\beta_{x x}+\beta_{z z}\right]^{2}\right)-e^{3}\right\}$ \\
\hline$f\left(\begin{array}{l}0 \overline{z \bar{x}} ; 0 \bar{z} \bar{y} \\
0 z^{*} x^{*} ; 0 z^{*} y^{*}\end{array}\right)$ & $\begin{array}{l}\left\{1-e^{1}\left(2+\beta_{x x}+\beta_{y y}+\beta_{x x} \beta_{y y}+\beta_{z z}\left[\beta_{x x}+\beta_{y y}+\beta_{z z}\right]\right)\right. \\
\left.+e^{2}\left(1+\beta_{x x}+\beta_{y y}+\beta_{x x} \beta_{y y}\right)\right\}\end{array}$ \\
\hline$f\left(\begin{array}{l}0 \bar{z} \bar{x} ; 0 \overline{z y} \\
0 z^{*} y^{*} ; 0 z^{*} x^{*}\end{array}\right)$ & $\begin{array}{l}\left\{-e^{1}\left(1-\beta_{x x}-\beta_{y y}+\beta_{x x} \beta_{y y}\right)\right. \\
\left.\quad+e^{2}\left(2-\beta_{x x}-\beta_{y y}+\beta_{x x} \beta_{y y}+\beta_{z z}\left[\beta_{x x}+\beta_{y y}+\beta_{z z}\right]\right)-e^{3}\right\}\end{array}$ \\
\hline$f\left(\begin{array}{l}0 \overline{y y} ; 0 \bar{z} \bar{x} \\
0 z^{*} x^{*} ; 0 z^{*} x^{*}\end{array}\right)$ & $\left(-e^{1}+e^{2}\right) \beta_{x y}\left(\beta_{x x}+\beta_{z z}\right)$ \\
\hline$f\left(\begin{array}{l}0 \bar{z} \bar{x} ; 0 \bar{z} \bar{x} \\
0 z^{*} x^{*} ; z_{z}^{*} y^{*}\end{array}\right)$ & $\left(-e^{1}+e^{2}\right) \beta_{y x}\left(\beta_{x x}+\beta_{z z}\right)$ \\
\hline$f\left(\begin{array}{l}0 \bar{z} \bar{x} ; 0 \bar{z} \bar{x} \\
0 z^{*} y^{*} ; 0 z^{*} y^{*}\end{array}\right)$ & $\left(-e^{1}+e^{2}\right) \beta_{y x}^{2}$ \\
\hline
\end{tabular}


TABLE 9 (continued)

\begin{tabular}{|c|c|}
\hline 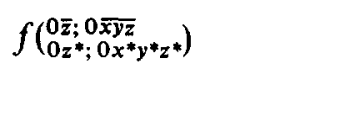 & $\begin{array}{l}\left\{1-e^{1}\left(2+\beta_{x x}+\beta_{y y}+\beta_{z z}\left[\beta_{x x}+\beta_{y y}+\beta_{z z}\right]\right)\right. \\
\left.+e^{2}\left(1+\beta_{x x}+\beta_{y y}\right)\right\}\end{array}$ \\
\hline$f\left(\begin{array}{l}0 \overline{x y z z} ; 0 \overline{x y z} \\
0 x^{*} y^{*} z^{*} ; 0 x^{*} y^{*} z^{*}\end{array}\right)$ & $\left\{\left(1-e^{1}\right)^{4}-e^{1}\left(1-e^{1}\right)^{2}\left[\beta_{x x}+\beta_{y y}+\beta_{z z}\right]^{2}\right\}$ \\
\hline$f\left(\begin{array}{l}0 ; 0 \bar{x} \overline{\overline{y z} z^{2}} \\
0 ; 0 x^{*} y^{*} z^{*} z^{* 2}\end{array}\right)$ & $\left\{1-e^{1}\left(1+\beta_{x x}+\beta_{y y}+\beta_{z z}+\frac{1}{2} \beta_{z z}^{2}\right)\right\}$ \\
\hline$f\left(\begin{array}{l}0 ; 0 \bar{z} \bar{x}(\bar{z} \bar{x}) \bar{z}^{2} \\
0 ; 0 z^{*} x^{*}\left(z^{*} x^{*}\right) z^{* 2}\end{array}\right)$ & $\left\{1-e^{1}\left(1+\beta_{x x}+\beta_{z z}+\beta_{x x} \beta_{z z}+\frac{1}{2} \beta_{z z}^{2}\right)\right\}$ \\
\hline$f\left(\begin{array}{l}0 ; 0 \bar{x} \overline{x y z} z^{2}(\bar{z} x) \\
0 ; 0 z^{*} x^{*} y z^{* 2}\left(z^{*} x^{*}\right)\end{array}\right)$ & $\left\{1-e^{1}\left(1+\beta_{x x}+\beta_{y y}+\beta_{z z}+\beta_{x x} \beta_{z z}+\frac{1}{2} \beta_{z z}^{2}\right)\right\}$ \\
\hline$f\left(\begin{array}{l}0 ; 0 ; 0 \overline{z x \bar{x}^{2}} \\
0 ; 0 z^{*} x^{*}\left(z^{*} x^{*}\right) z^{* 2}\end{array}\right)$ & $e^{1}\left[\frac{A}{f(A-f)}\right]^{\frac{1}{2}} \bar{K}_{z} \beta_{x y}$ \\
\hline 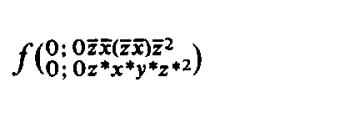 & $e^{1} \beta_{y x}\left[\frac{A}{f^{\prime}\left(A-f^{\prime}\right)}\right]^{\frac{1}{z}} K_{z}^{*}$ \\
\hline$f\left(; 0 z^{*} x^{*} y^{*} z^{* 2}\right)$ & 1 \\
\hline$f\left(\begin{array}{l}0 \overline{x y z} ; 0 \overline{x y} \\
0 x^{*} y^{*} z^{*} ; 0 z^{*} x^{*}\end{array}\right)$ & $\begin{array}{l}\left\{\left(1-e^{1}\right)^{3}-\beta_{y y} e^{1}\left(1-e^{1}\right)^{2}\right. \\
\left.-e^{1}\left(1-e^{1}\right)\left(\beta_{x x}+\beta_{z z}\right)\left(\beta_{x x}+\beta_{y y}+\beta_{z z}\right)\right\}\end{array}$ \\
\hline$f\left(\begin{array}{l}0 ; 0 \overline{x y z z^{2}}(\overline{z x})(\bar{z} y) \\
0 ; 0 x^{*} y^{*} z^{*} z^{*}\left(z^{*} x^{*}\right)\left(z^{*} y^{*}\right)\end{array}\right)$ & $\left\{1-e^{1}\left(1+\beta_{x x}+\beta_{y y}+\beta_{z z}+\beta_{z z}\left(\beta_{x x}+\beta_{y y}+\frac{1}{2} \beta_{z z}\right)\right\}\right.$ \\
\hline$f\left(\begin{array}{l}0 ; 0 \overline{x y z z^{2}}(\overline{z x}) \bar{x}^{2} \\
0 ; 0 x^{*} y^{*} z^{*} z^{*}+2\left(z^{*} x^{*}\right) x^{* 2}\end{array}\right)$ & $\left\{1-e^{1}\left(1+\beta_{x x}+\beta_{y y}+\beta_{z z}+\frac{1}{2}\left(\beta_{x x}+\beta_{z z}\right)^{2}\right)\right\}$ \\
\hline
\end{tabular}

while particles $1, \ldots, l^{\prime}$ belong to fragment $f^{\prime}$, particles $l^{\prime}+1, \ldots, l^{\prime}+n^{\prime}=l+n$ to fragment $A-f^{\prime}$. E.g.

$$
\begin{aligned}
& F\left(\begin{array}{c}
0 \bar{z} \bar{x} ; \mathbf{x} \bar{z} \bar{x} \\
0 ; 0 z^{*} x^{*} y^{*} z^{* 2}
\end{array}\right) \equiv\left[\frac{\partial}{\partial \bar{k}_{2 z}} \frac{\partial}{\partial \bar{k}_{3_{x}}} \frac{\partial}{\partial \bar{k}_{5_{z}}} \frac{\partial}{\partial \bar{k}_{6_{x}}} \frac{\partial}{\partial k_{3_{z}}^{*}} \frac{\partial}{\partial k_{4_{x}}^{*}}\right. \\
& \left.\times \frac{\partial}{\partial k_{5_{y}}^{*}} \frac{\partial^{1}}{\sqrt{2 !}} \frac{\partial^{2}}{\partial k_{6_{z}}^{* 2}}\left\{\sum_{\boldsymbol{P}}(-1)^{\sigma(\boldsymbol{P})} \exp \left[\sum_{i=1}^{6}\left(\overline{\boldsymbol{k}}_{i} P k_{i}^{*}\right)\right]\right\}\right]_{\overline{\mathbf{k}}_{i}=\mathbf{0}, \mathbf{K}_{\mathbf{j}}^{*}=0, \boldsymbol{k}_{\mathrm{c} \cdot \mathbf{m} .}=0} \\
& \equiv \mathrm{e}^{\eta\left(\overline{\mathbf{K}} \cdot \mathbf{K}^{* *}\right)} f\left(\begin{array}{c}
0 \bar{z} \bar{x} ; 0 \bar{z} \bar{x} \\
0 ; 0 z^{*} x^{*} y^{*} z^{* 2}
\end{array}\right),
\end{aligned}
$$

where

$$
\eta=\frac{l^{\prime}(A-f)-l f^{\prime}+(l+n)\left(f f^{\prime} / A\right)}{\left[f(A-f) f^{\prime}\left(A-f^{\prime}\right)\right]^{\frac{1}{2}}},
$$

and with $\sum l^{\prime}=f^{\prime}, \sum l=f, \sum(l+n)=A,\left(\sum=\right.$ sum over columns), the product of single-column factors $\exp \eta\left(\widetilde{\boldsymbol{K}}^{*} \cdot \overline{\boldsymbol{K}}^{*}\right)$ combine to make the factor

$$
\prod_{\text {columns }} \mathrm{e}^{n\left(\overline{\mathbf{K}} \cdot \mathbf{K}^{*}\right)}=\exp \left\{\frac{f^{\prime}(A-f)}{\left[f(A-f) f^{\prime}\left(A-f^{\prime}\right)\right]^{\frac{1}{2}}}\left(\overline{\mathbf{K}} \cdot \boldsymbol{K}^{*}\right)\right\}
$$

for the full 4-columned tableau. 
Table 10

The $f$-functions with $l \neq l, n \neq n^{\prime}$

$f\left(\begin{array}{c}0 \bar{z} \bar{x} ; 0 \bar{z} \bar{x} \\ 0 z ; ; x ; 0 y * z\end{array}\right)$

$-\left(1-e^{1}\right)^{2} \beta_{y x}$

$f\left(\begin{array}{l}0 \overline{z y} ; 0 \overline{z y} \\ 0 z^{*} ; x^{*} 0 y^{*} z\end{array}\right)$

$\left(1-e^{1}\right)^{2} \beta_{x y}$

$f\left(\begin{array}{l}0 \bar{z} \bar{x} ; 0 \overline{z y} \\ 0 z^{*} ; x^{*} 0 y^{*} z\end{array}\right)$

$\left\{-\left(1-\beta_{x x}\right)+e^{1}\left(2-\beta_{x x}+\beta_{y y}+\beta_{z z}\left[\beta_{x x}+\beta_{y y}+\beta_{z z}\right]\right)\right.$

$\left.-e^{2}\left(1+\beta_{y y}\right)\right\}$

$f\left(\begin{array}{l}0 \overline{z y} ; 0 \bar{z} \bar{x} \\ 0 z ; ; x ; * y *\end{array}\right)$

$\left\{\left(1-\beta_{y y}\right)-e^{1}\left(2+\beta_{x x}-\beta_{y y}+\beta_{z z}\left[\beta_{x x}+\beta_{y y}+\beta_{z z}\right]\right)\right.$

$\left.+e^{2}\left(1+\beta_{x x}\right)\right\}$

$f\left(\begin{array}{c}0 \bar{x} \bar{x} ; 0 \bar{z} \bar{x} \\ 0 ; z^{*} x^{*} 0 y^{*} z+2\end{array}\right)$

$-\sqrt{2}\left(1-e^{1}\right)\left[\frac{A}{f(A-f)}\right]^{\frac{1}{2}} \bar{K}_{z} \beta_{y x}$

$f\left(\begin{array}{l}0 ; \bar{z} ; 0 \overline{z y} \\ 0 ; z^{*} x^{*} 0 y^{*} z * 2\end{array}\right)$

$\sqrt{2}\left(1-e^{1}\right)\left[\frac{A}{f(A-f)}\right]^{\frac{1}{2}} \bar{K}_{z} \beta_{x y}$

$f\left(\begin{array}{l}0 \bar{z} \bar{x} ; 0 \bar{z} \bar{y} \\ 0 ; z^{*} x x^{*} 0 y^{*} z^{* 2}\end{array}\right)$

$\left[\frac{1}{2} \frac{A}{f(A-f)}\right]^{\frac{1}{2}} \bar{K}_{z}\left\{\left(-2+2 \beta_{x x}+\beta_{z z}\right)+e^{1}\left(2+2 \beta_{y y}+\beta_{z z}\right)\right\}$

$f\left(\begin{array}{l}0 \overline{z y} ; 0 \bar{z} \bar{x} \\ 0 ; z^{*} x^{*} 0 y^{*} z * 2\end{array}\right)$

$\left[\frac{1}{2} \frac{A}{f(A-f)}\right]^{\frac{1}{z}} \bar{K}_{z}\left\{\left(2-2 \beta_{y y}-\beta_{z z}\right)-e^{1}\left(2+2 \beta_{x x}+\beta_{z z}\right)\right\}$

$f\left(\begin{array}{l}0 \bar{z} \bar{x} ; 0 \bar{z} \bar{x} \\ 0 ; z^{*} x^{*} 0\left(z^{*} x^{*}\right) z^{* 2}\end{array}\right)$

$\frac{1}{\sqrt{2}}\left(\frac{A}{f(A-f)}\right) \bar{K}_{z}^{2}\left\{\left(-2+\beta_{x x}+\beta_{z z}\right)+e^{1}\left(2+\beta_{x x}+\beta_{z z}\right)\right\}$

$f\left(\begin{array}{l}0 \bar{z} \bar{x} ; 0 \bar{y} \bar{y} \\ 0 ; z^{*} x^{*} 0\left(z^{*} x^{*}\right) z * z\end{array}\right)$

$\frac{1}{\sqrt{2}}\left(\frac{A}{f(A-f)}\right) \bar{K}_{z}^{2} e^{1} \beta_{x y}$

$f\left(\begin{array}{l}0 \overline{z y} ; 0 \bar{z} \bar{x} \\ 0 ; z^{*} x^{*} 0\left(z^{*} x^{*}\right) z^{* 2}\end{array}\right)$

$\frac{1}{\sqrt{2}}\left(\frac{A}{f(A-f)}\right) \bar{K}_{z}^{2} \beta_{x y}$

$f\left(\begin{array}{l}0 \overline{z y} ; 0 \overline{z y} \\ 0 ; z^{*} x^{*} 0\left(z^{*} x^{*}\right) z^{* 2}\end{array}\right)$

0

$f\left(\begin{array}{l}0 \bar{z} ; 0 \overline{x y z} \\ 0 ; z^{*} 0 x^{*} y^{*} z^{* 2}\end{array}\right)$

$\left[\frac{1}{2} \frac{A}{f(A-f)}\right]^{\frac{1}{2}} \bar{K}_{z}\left\{\left(2-\beta_{z z}\right)-e^{1}\left(2+2 \beta_{x x}+2 \beta_{y y}+\beta_{z z}\right)\right\}$

$f\left(\begin{array}{l}0 \bar{z} ; 0 \overline{x y z} \\ 0 ; z^{*} 0 x^{*}\left(z^{*} x^{*}\right) z^{* 2}\end{array}\right)$

$\frac{1}{\sqrt{2}}\left(\frac{A}{f(A-f)}\right) \bar{K}_{z}^{2} e^{1} \beta_{x y}$

$f\left(\begin{array}{l}0 \bar{z} ; 5 \overline{y z} 0 \\ ; 0 z^{*} x^{*} y^{*} z^{* 2}\left(z^{*} x^{*}\right)\end{array}\right)$

$-\frac{1}{\sqrt{2}}\left[\frac{A}{f(A-f)}\right]^{\frac{3}{2}} \bar{K}_{z}^{2} \bar{K}_{x}$

$f\left(\begin{array}{l}0 \bar{x} \bar{x} ; 0 \bar{x} \bar{x} \\ ; 0 z^{*} x^{*} y^{*} z^{* 2}\left(z^{*} x^{*}\right)\end{array}\right)$

$\sqrt{2}\left[\frac{A}{f(A-f)}\right]^{\frac{3}{2}} \bar{K}_{z}^{2} \bar{K}_{y}$ 
TABLE 10 (continued)

$f\left(\begin{array}{l}0 \bar{z} \bar{x} ; 0 \overline{z y} \\ ; 0 z^{*} x^{*} y^{*} z^{* 2}\left(z^{*} x^{*}\right)\end{array}\right)$

$-\frac{1}{\sqrt{2}}\left[\frac{A}{f(A-f)}\right]^{\frac{3}{2}} \bar{K}_{z}^{2} \bar{K}_{x}$

$f\left(\begin{array}{l}0 \overline{z y} ; 0 \bar{z} \bar{x} \\ ; 0 z^{*} x^{*} y^{* 2}\left(z^{*} x^{*}\right)\end{array}\right)$

$-\frac{1}{\sqrt{2}}\left[\frac{A}{f(A-f)}\right]^{\frac{3}{2}} \bar{K}_{z}^{2} \bar{K}_{x}$

$f\left(\begin{array}{l}0 \bar{z} \overline{x y z} \bar{z}^{2} ; 0 \\ ; 0 z^{*} x^{*} y^{*} z^{* 2}\left(z^{*} x^{*}\right)\end{array}\right)$

$\left(\frac{A}{f(A-f)}\right) \bar{K}_{z} \bar{K}_{x}$

$f\left(\begin{array}{l}0 \overline{x y z} ; 0 \overline{x y z} \\ 0 ; x^{*} y^{*} z=z^{* 2}\left(z^{*} x^{*}\right) x^{* 2}\end{array}\right)$

0

$f\left(\begin{array}{c}0 \overline{x y y} ; 0 \overline{x y z} \\ 0 ; x^{*} y^{*} z^{*} 0 z^{* 2}\left(z^{*} x^{*}\right)\left(z^{*} y^{*}\right)\end{array}\right)$

$\sqrt{2}\left[\frac{A}{f(A-f)}\right]^{\frac{3}{2}} K_{z}^{3}\left\{\left(1-e^{1}\right)-\frac{1}{2}\left(1+e^{1}\right)\left(\beta_{x x}+\beta_{y y}+\beta_{z z}\right)\right\}$

The $f(\ldots \ldots \ldots)$ are tabulated in terms of the quantities $e^{p}$ and $\beta_{i j}$, defined by

$$
\begin{gathered}
e^{p} \equiv \exp \left\{-p \frac{A}{\left[f(A-f) f^{\prime}\left(A-f^{\prime}\right)\right]^{\frac{1}{2}}}\left(\overline{\boldsymbol{K}} \cdot \boldsymbol{K}^{*}\right)\right\}, \\
\beta_{z x} \equiv\left[\frac{A}{f(A-f)}\right]^{\frac{1}{2}} \bar{K}_{z}\left[\frac{A}{f^{\prime}\left(A-f^{\prime}\right)}\right]^{\frac{1}{2}} K_{x}^{*} .
\end{gathered}
$$

Note that:

$$
\beta_{z x} \beta_{x z}=\beta_{z z} \beta_{x x}
$$

Note added in proof: Norm kernels for some complex cluster systems have recently also been worked out by $\mathrm{Y}$. Fujiwara and $\mathrm{H}$. Horiuchi who give norm kernels for ${ }^{12} \mathrm{C}+2 \alpha,{ }^{12} \mathrm{C}+{ }^{8} \mathrm{Be},{ }^{12} \mathrm{C}+{ }^{12} \mathrm{C}, 2 \alpha+{ }^{20} \mathrm{Ne},{ }^{8} \mathrm{Be}+{ }^{8} \mathrm{Be}$, and $\alpha+{ }^{24} \mathrm{Mg}$ systems, (to be published). We are indebted to these authors for pointing out a small numerical error in our original preprint.

\section{References}

1) K. Wildermuth and Y. C. Tang, A unified theory of the nucleus (Vieweg, Braunschweig, 1977)

2) H. H. Hackenbroich, Symp. on present status and novel developments in the nuclear many-body problem, Rome (Editrice, Bologna, 1973)

3) Y. C. Tang, M. LeMere and D. R. Thompson, Phys. Reports 47 (1978) 167

4) W. Schütte, Ph. D. Thesis, Cologne (1976)

5) H. Stöwe and W. Zahn, Nucl. Phys. A286 (1977) 89; A289 (1977) 317; Z. Phys. A286 (1978) 173; J. Phys. G4 (1978) 1423

6) W. Zahn, Nucl. Phys. A269 (1976) 138

7) H. H. Hackenbroich, T. H. Seligman and W. Zahn, Nucl. Phys. A259 (1976) 445

8) H. Friedrich and K. Langanke, Nucl. Phys. A252 (1975) 47

9) H. Friedrich, K. Langanke, A. Weiguny and R. Santo, Phys. Lett. 55B (1975) 345

10) H. Friedrich, K. Langanke and A. Weiguny, Phys. Lett. 63B (1976) 125 
11) D. L. Hill and J. A. Wheeler, Phys. Rev. 89 (1953) 1102

12) W. Bargmann, Commun. Pure and Appl. Math. 14 (1961) 187

13) I. E. Segal, Illinois J. Math. 14 (1962) 500

14) A. O. Barut and L. Girardello, Commun. Math. Phys. 21 (1971) 41

15) W. Sünkel and K. Wildermuth, Phys. Lett. 41B (1972) 439

16) H. Horiuchi, Progr. Theor. Phys. 47 (1972) 1058; 55 (1976) 1448

17) H. Friedrich, Nucl. Phys. A224 (1974) 537

18) P. Kramer, M. Moshinsky and T. H. Seligman, in Group theory and its applications IIl, ed. E. M. Loebl (Academic, New York, 1975)

19) W. Zahn, Burg monographs in science, Vol. 2 (Burg, Basel, 1975)

20) H. H. Hackenbroich, T. H. Seligman and W. Zahn, Helv. Phys. Acta 50 (1977) 723

21) D. M. Brink, Int. School of Physics Enrico Fermi, Course 36 (1965) 247

22) T. H. Seligman and W. Zahn, J. Phys. G2 (1976) 79

23) K. T. Hecht and W. Zahn, Lecture notes in physics 94 (Springer, Berlin, 1979)

24) K. T. Hecht and W. Zahn, Nucl. Phys. A318 (1979) 1

25) K. T. Hecht and W. Zahn, Nucl. Phys. A313 (1979) 77

26) K. T. Hecht and W. Zahn, Nukleonika (1979), in press

27) Y. Fujiwara and H. Horiuchi, Prog. Theor. Phys. 63 (1980) 895

28) M. G. Mazarakis and W. E. Stephens, Phys. Rev. C7 (1973) 1280

29) W. Galster, W. Treu, P. Dück, H. Fröhlich and H. Voit, Phys. Rev. C15(1977) 950; Phys. Lett. 67B (1977) 26; Phys. Rev. C18 (1978) 2148

30) T. M. Cormier, C. M. Jachcinski, G. M. Berkowitz, P. Braun-Munzinger, P. M. Cormier, M. Gai, J. W. Harris, J. Barrette and H. E. Wegner, Phys. Rev. Lett. 40 (1978) 924

31) N. R. Fletcher, J. D. Fox, G. J. Kekelis, G. R. Morgan and G. A. Norton, Phys. Rev. C13(1976) 1173

32) K. U. Kettner, H. Lorenz-Wirzba and C. Rolfs. Z. Phys. A298 (1980) 65

33) H. W. Becker, Diplomarbeit, Universität Münster (1978)

34) H. Horiuchi, Prog. Theor. Phys. 51 (1974) 745, Prog. Theor. Phys. Suppl. 62 (1977) 90

35) J. P. Elliott, Proc. Roy. Soc. A245 (1958) 128; 562

36) J. P. Draayer and Y. Akiyama, J. Math. Phys. 14 (1973) 1904, Comp. Phys. Comm. 5 (1973) 405

37) D. A. Bromley, J. A. Kuehner and E. Almquist, Phys. Rev. Lett. 4 (1960) 365

38) R. L. Phillips, K. A. Erb, D. A. Bromley and J. Weneser, Phys. Rev. Lett. 42 (1979) 566

39) L. E. Cannell, R. W. Zurmühle and D. P. Balamuth, Phys. Rev. Lett. 43 (1979) 837

40) W. A. Friedman, K. W. McVoy and M. C. Nemes, Phys. Lett. 87B (1979) 179

41) B. Imanishi, Nucl. Phys. A125 (1969) 33

42) W. Scheid, W. Greiner and R. Lemmer, Phys. Rev. Lett. 25 (1970) 176

43) H. J. Fink, W. Scheid and W. Greiner, J. Phys. G1 (1975) 685

44) J. Y. Park, W. Scheid and W. Greiner, Phys. Rev. C10 (1974) 967; C16 (1977) 2276

45) Y. Kondō, Y. Abe and T. Matsuse, Phys. Rev. C19 (1979) 1356; 1365

46) J. F. Berger and D. Gogny, Nucl. Phys. A333 (1980) 302

47) A. Tohsaki-Suzuki, Fizika 9 Suppl. 4 (1977) 183

48) K. T. Hecht and S. C. Pang, J. Math. Phys. 10 (1969) 1571

49) A. Antillón and T. H. Seligman, to be published

50) M. Hamermesh, Group theory and its application to physical problems (Addison-Wesley, Reading, Mass., 1962) 231

51) Y. Suzuki, Progr. Theor. Phys. 50 (1973) 341 ; 55 (1976) 1751

52) P. Kramer and T. H. Seligman, Nucl. Phys. A136 (1969) 545; A186 (1972) 45

53) E. J. Reske, Univ. of Michigan Internal Report No. 258 (1980)

54) K. T. Hecht and D. Braunschweig, Nucl. Phys. A244 (1975) 365

55) J. P. Draayer, Nucl. Phys. A237 (1975) 157

56) T. Fliessbach, Z. Phys. A288 (1978) $211 ; 219 ;$ A294 (1980) 79

57) K. T. Hecht, Nucl. Phys. 62 (1965) 1

58) A. R. Edmonds, Angular momentum in quantum mechanics (Princeton Univ., Princeton, 1957)

59) K. T. Hecht, Nucl. Phys. A283 (1977) 223

60) L. C. Biedenharn and J. D. Louck, J. Math. Phys. 13(1972) 1985; 12 (1971) 173; 11 (1970) 2368;

L. C. Biedenharn, J. D. Louck, E. Chacón and M. Ciftan, J. Math. Phys. 13 (1972) 1957;

J. D. Louck, Am. J. Phys. 38 (1970) 3

61) D. J. Millener, J. Math. Phys. 19 (1978) 1513 